\title{
SIMULATION OF MULTICOMPONENT AEROSOL DYNAMICS
}

\author{
Thesis by \\ Yong Pyo Kim \\ In Partial Fulfillment of the Requirements \\ for the Degree of \\ Doctor of Philosophy
}

California Institute of Technology

Pasadena, California

1992

(Submitted August 23, 1991) 


\section{Acknowledgment}

I want to thank my advisor, John H. Seinfeld, for all the contributions that he has made to my research.

I also thank Rick Flagan, George Gavalas, and Kikuo Okuyama for taking time to exchange ideas with me about my research.

I also wish to thank David Huang, Shih Chen Wang, Tony Wexler, Spiros Pandis, Barbara Wyslouzil, and Chak Chan for their help.

I have enjoyed the life at CALTECH and I want to thank all those who made this possible; my parents, Chang Koo Yun, In Seok Kang, and the Suk Woo Nam family.

Last but not least, I am grateful to Meeyoung for too many things to mention here, and to Hearam for giving me a very important reason to live. 


\section{Abstract}

Chemical composition and, therefore, several physical properties, such as refractive index or density, of an aerosol system may be distributed in one particle size. The effect of these particles of the same size but different properties, so-called mixed particles, on aerosol dynamics can be important. Several aspects of the number-property distribution; effect on aerosol dynamics and importance, definition, state of the art of the measurement technique, available data, and numerical schemes are discussed and further research directions are discussed.

The moving sectional method is extended to simulate multicomponent aerosol dynamics resulting from condensation/ evaporation processes. This method uses a Lagrangian approach in which section boundaries and component masses in a section vary according to the characteristics of condensation/ evaporation rates while conserving number concentration in a section throughout the simulation. Simulation of model problems for which new analytical solutions have been obtained shows excellent agreement with the analytical solutions. Limitations and applicability of the sectional method are discussed.

A technique for direct numerical solution of the multicomponent aerosol general dynamic equation is developed and tested. The method obtains the aerosol sizecomposition distribution without the need to make any a priori assumptions about the nature of the distribution. Numerical solutions are compared with analytical solutions for model problems of pure condensation/evaporation, pure coagulation, and simultaneous condensation and coagulation. The advantages, applicability, and the limitations of the approach are discussed.

An analysis of the tandem differential mobility analyzer (TDMA) is prorsed in which the conditioner between the two DMAs is simulated by the multicomponent aerosol general dynamic equation (GDE). The use of the TDMA to separate an externally mixed aerosol is illustrated by simulating the data of Liu et al. (1978).

Numerical issues in grid-based photochemical air quality models are reviewed. Numerical schemes for advection and chemical kinetics in gas-phase and for dynamics 
in aerosol-phase are compared.

Finally, a numerical code is developed based on direct numerical solution of the multicomponent aerosol general dynamic equation. 


\section{Contents}

$\begin{array}{ll}\text { Acknowledgment } & \text { ii }\end{array}$

Abstract

Contents

I The Number-Property Distribution of an Aerosol 1

1 Introduction 2

2 Definition $\quad 5$

3 Experimental Methods $\quad 14$

4 Numerical Simulation $\quad 20$

5 The Remainder of this Thesis $\quad 27$

II Simulation of Multicomponent Aerosol Condensation by the Moving Sectional Method 44

1 Introduction $\quad 45$

2 General Dynamic Equation for Condensation/Evaporation 48

3 Sectional Method $\quad 49$

4 Analytical Solutions for Multicomponent Aerosol Condensation/Evaporation $\quad 52$

5 Numerical Solutions of Multicomponent Aerosol Condensation/Evaporation $\quad 53$

6 Conclusion $\quad 57$

Appendix. Analytical Solution for Multicomponent Aerosol Condensation/Evaporation

III Simulation of Multicomponent Aerosol Dynamics

1 Introduction $\quad 75$

2 Multicompoient Aerosoi General Dynamic Equation $\quad 77$

3 Numerical Methods for Aerosol Dynamics $\quad 80$

4 Numerical Solution of Multicomponent Aerosol Dynamics 89

5 Discussion $\quad 100$

6 Conclusions 105 
IV Use of the Tandem Differential Mobility Analyzer to Evaluate the Degree of Mixing of Aerosol Particles

1 Introduction

2 TDMA Analysis

3 Application of the TDMA to Determine Aerosol Mixedness 143

4 Conclusion

V Conclusions

1 Summary

2 Recommendations for Future Research

\section{A.I Numerical Issues in Grid-Based Photochemical}

Air Quality Models

Part A Numerical Aspects of Gas-Phase Photochemical Air Quality Models

A.1 Advection

A.2 Chemical Kinetics

A.3 Summary of Numerical Issues in Gas-Phase Photochemical Air Quality Models

Part B Numerical Aspects of Atmospheric Aerosol Models

B.1 The General System

B.2 Numerical Solution of the Single Component Aerosol General Dynamic Equation

B.3 Numerical Solution of the Multicomponent Aerosol General Dynamic Equation

B.4 Numerical Issues in Episodic $\mathrm{PM}_{10}$ Models

1 Introduction

210

2 Processes and Mechanisms

210

3 Input Data File

214 
Chapter I

\section{THE NUMBER-PROPERTY DISTRIBUTION OF AN AEROSOL}




\section{INTRODUCTION}

Aerosols that consist of more than one chemical component frequently exhibit variations of composition among particles of the same size. These variations may play an important role in multicomponent aerosol dynamics and apparent aerosol properties. Heintzenberg and Covert (1) reviewed the importance of the properties of individual airborne particles at a given size on the chemical and physical processes occurring in the atmosphere. Due to the wide variation of the atmospheric sources and transport routes (2), it is natural that atmospheric aerosols consist of various chemical compounds with overlapping distributions.

A frequently cited property, for example, that shows the importance of the numberproperty distribution is hygroscopicity (3). Consider particles of the same size consist. ing of two chemical compounds; one is hygroscopic while the other is non-hygroscopic. After humidification, an aerosol system of pure hygroscopic particles and pure nonhygroscopic particles (the right side of Figure 1) will develop into two particle size's while a system in which individual particles are the equal mixture of two compounds (the left side of Figure 1) will give only one particle size.

Another important example are the optical property differences. In the case of particles of the same size consisting of light absorbing and non-absorbing chemical compounds, as shown in Figure 2, the amount of light absorbed by an aerosol system consisting of pure light absorbing particles and pure non-absorbing particles can be different from that by an aerosol system consisting of particles in which two compounds are equally mixed in each particle (1). The dependence of optical properties on the number-size distribution has been recognized, as has been the variation of optical properties with the average chemical composition. But these dependencies could not explain the difference of optical property between two sets of particles of the same size range and the same average chemical composition.

Finally, another well known property variation of particles of the same size is the charge distribution; the distribution of electrical charges among particles of the same size has been recognized very early and certain distributions were proposed, 
for example, the Boltzmann and the Fuchs distribution. This charge distribution is important in several areas; particle removal by means of electrical methods (4) and classification of the number-size distribution by electric mobility (5).

Historically, the first quantity measured from an aerosol sample was the total concentrations; for example, the mass concentration of total suspended particulate (TSP) was used for regulatory purposes (6). With the development of measurement techniques, the importance of the number-size distribution was recognized. All aerosol properties are dependent on the size of particles, directly or indirectly. The so-called PM-10, for example, particulate matter up to the nominal aerodynamic diameter of 10 $\mu \mathrm{m}$ with a $50 \%$ cutoff efficiency, is now used instead of the TSP (6-8) for regulatory purpose in most countries. Even the vapor pressure of a chemical species over a curved surface of an aerosol is different from that over the bulk flat surface and varies with the particle size, and subsequently condensation/evaporation rates also vary (9). Furthermore, it was recognized that chemical composition and morphology of particles also play important roles in aerosol dynamics and apparent properties determination. At first, only average compositions were measured as with the total concentration, usually by the wet analyses (10), but with the development of number-size distribution measurements, average compositions at each size range were accessible. However, it was soon recognized that average composition and the number-size distribution do not explain atmospheric aerosol measurement data accurately. Hidy (11) presented a brief history of atmospheric aerosol characterization.

One way of looking at property variation is the concept of mixed particles, a mixture of several chemical species, or properties in particles of the same size (3). One extreme case is internally mixed particles in which all particles have the same composition, or property. The other extreme is externally mixed particles in which all particles consist of pure species or properties and are present in appropriate numbers. In addition, there is, naturally, a distribution of possible intermediate situations (12). The concept of mixed particles has been used to consider atmospheric aerosol problems. For example, the mixedness of particles is thought to be linked to the age of 
aerosols and the relative degree of gas-to-particle conversion and coagulation.

An important and difficult problem has been how to measure or to recognize the number-property distribution. Research efforts have resulted in a few measurement methods that can measure chemical/physical property variation of same size particles. One class of methods measures properties of individual particles directly. Direct measurement of individual particles provides elemental analysis (quantitative or qualitative), particle size, and in some methods, particle morphology and is the most convincing proof that property variation exists, and a huge improvement of the measurement techniques has been achieved. It, however, is still far from for the routine analysis and has several limitations to be overcome. Another class of methods exploits the property differences (usually the difference of one property) to measure property variation. The difference of a property can be used to detect and/or separate mixed particles by either using a series of instruments which are operated by different rinciples or inducing changes of the distribution according to the property difference. While methods based on this principle are powerful tools to study a property difference, it is hard to recognize the difference of several properties by using these methods.

If the number of interesting properties or chemical species becomes large and the interaction between them becomes complicated, it is difficult, if not impossible, to measure, understand, and exploit the number-property distributions experimentally. In that case, numerical simulation is the only viable option to study the numberproperty distribution. As with the development of the measurement methods, numerical routines have started from the prediction of the total quantities based on the assumed number-size distribution with adjustable parameters. Then, the simulation of the number-size distribution of the single component aerosol system based on the aerosol general dynamic equation (GDE) followed. There has been considerable progress in developing multicomponent aerosol dynamics simulators recently and now a few numerical routines are available for simulating multicomponent aerosol dynamics. 
There are several general references $(4,9,13-16)$ that deal with aspects of the aerosol system, but few deal with the number-property distribution extensively. Therefore, this review can be regarded as an introduction to the number-property distribution, especially the number-chemical composition distribution, in the aerosol system. The goals of this review are: (1) to discuss definitions representing property variation of particles of the same size; (2) to discuss the mixedness of particles; (3) to summarize the state of the art of measurement methods and measurement data; (4) to summarize the state of the art of numerical routines for simulating multicomponent aerosol dynamics; and (5) to identify future research needs on property variation.

\section{DEFINITION}

\subsection{Definition of a Distribution.}

There are a spectrum of ways to define a distribution of a multicomponent aerosol system. The simplest one is the number distribution on a one-dimensional domain and the most complicated one is the number distribution on the multidimensional domain. Other distributions, such as surface area, mass, or volume, are all based on the number distribution and can be derived easily from.

The number distribution on the one-dimensional domain can be described as

$$
n(x, t) \quad \text { or } \quad \frac{d N(x, t)}{d x}
$$

where $x$ is the independent variable in mathematical terms or the property of interest in physical terms, and $n(x, t)$ is the distribution function at a time $t$ such that $n(x, t) d x=d N(x, t)$ is the number concentration of particles having $x$ in the range $[x, x+d x]$.

Any property that is of interest can be used as the independent variable to describe the behavior of the aerosol system. For example, the refractive index of the particles may be used as the independent variable if the optical effect is important or the amount of water in the particles can be used if the water content is of prime concern. 
But the most widely used and arguably the most important one is the size: diameter, volume, or mass, of an aerosol (usually simply called the size distribution) since practically all aerosol properties depend strongly on particle size.

This representation is the simplest one to describe the aerosol system and still is the most widely used one since it is important, easy to implement, and requires the least computational burden compared to other definitions. Also, important quantities, total concentrations and mean values, are easily calculated from the so-called moment relations;

$$
M_{w}=\int_{-\infty}^{\infty} w(x) n(x, t) d x
$$

where $w(x)$ is the weight function. Thus, the total quantities are integral propertir. of the number-size distribution. Some examples of the moment relations based un the number-size distribution are shown in Table $I$.

The one-dimensional domain representation is valid, trivially, if an aerosol consinof a single component and is of spherical shape. It is also valid for the multicompn. nent aerosol system if only one property is of interest and used as the independent variable; particle size is an example. The number-size distribution is widely used for classification of particles experimentally and numerically since, still, most data are obtained by instruments that can resolve only particle size.

The limitation of this approach using the number distribution on the one-dimensional domain for the multicomponent aerosol system is self-evident. For a fixed value of one property, there may be distribution or variation of other properties. Moreover, if the effects of other properties are not negligible, this approach cannot fully describe the system.

A definition that accounts for composition variations on the one-dimensional domain was developed in the so-called multicomponent sectional representation (17). In this definition, a one-dimensional domain, particle mass or volume, is divided into a finite number of sections in each of which the total mass distribution is assumed to be uniform, as are the mean mass fractions of each component in the section. With 
some derivation, the total number concentration distribution and the components' mean mass fraction distributions are obtained on the particle size domain.

This definition has several advantages: (1) it can describe mass concentration for each component on the particle size domain; and (2) the computational requirements are tractable since it uses a one-dimensional domain. This definition can describe variation of each component mass concentration with the particle size with only a small increase of the computational requirement to that of the number distribution on the one-dimensional domain. This definition is suitable for describing the number-size distribution measurement with chemical analysis for the each size range. Numerical routines based on this definition have been applied to several areas which will be discussed later.

The sectional representation is strictly valid if all particles in the one size range have the same compositions. Such internally mixed particles may exist for wellaged atmospheric particles since coagulation mixes particles of different sizes and compositions and averages out the differences. But, usually, this representation is only an extreme case of the general aerosol system. Thus, this definition has one serious limitation; namely, only the mean mass fractions of each component in the section are known, composition variations in the section are not considered (17). Therefore, actually all particles in one section are calculated as having the same compositions, i.e., are regarded as internally mixed particles.

The most general form of the distribution is the number distribution on the multidimensional domain;

$$
n\left(x_{1}, x_{2}, \ldots, x_{s}, t\right) \quad \text { or } \quad \frac{\partial N\left(x_{1}, x_{2}, \ldots, x_{s}, t\right)}{\partial x_{1} \partial x_{2} \ldots \partial x_{s}}
$$

where $x_{i}$ is the $i$-th property of interest and $n$ or $\frac{d N}{d x_{1} d x_{2} \ldots d x_{o}}$ is the number density distribution function such that $n\left(x_{1}, x_{2}, \ldots, x_{s}, t\right) d x_{1} d x_{2} \ldots d x_{s}$ is the number concentration of particles having properties in the range $\left[x_{i}, x_{i}+d x_{i}\right], i=1, s$. The number and the choice of the independent variables are arbitrary as long as these are independent. 
An obvious choice of the independent variables is the chemical composition of the aerosol (18),

$$
n\left(m_{1}, m_{2}, \ldots, m_{s}, t\right) \quad \text { or } \quad \frac{\partial N\left(m_{1}, m_{2}, \ldots, m_{s}, t\right)}{\partial m_{1} \partial m_{2} \ldots \partial m_{s}}
$$

where $m_{i}$ is the mass of the $i$-th component and $s$ is the number of the species. The distribution on the chemical composition domain can explain many aerosol properties which cannot be fully explained by the definitions based on the one-dimensional domain.

Another choice is the particles size together with other properties. For example, a number-chemical composition distribution can be expressed as

$$
n\left(m_{1}, m_{2}, \ldots, m_{s-1}, M, t\right) \quad \text { or } \quad \frac{\partial N\left(m_{1}, m_{2}, \ldots, m_{s-1}, M, t\right)}{\partial m_{1} \partial m_{2} \ldots \partial m_{s-1} \partial M}
$$

where $M$ is the total mass of a particle, $M=\sum_{i=1}^{s} m_{i}$. Also, properties other than the chemical composition can be used without loss of generality as long as the variables are linearly independent. This kind of expression can be used for the general multicomponent aerosol system but is usually used for cases of one property variation in particles of the same size, in practice, since to choose multiple properties that are linearly independent is difficult both theoretically and experimentally. See, for example, Heintzenberg and Covert (1) for number distribution on size-one property domains. This kind of three-dimensional representation is practically useful to study the property of interest, but is not satisfactory if there are more than one interesting properties.

So far, it is implicitly assumed that particles are spherical. Particles of the same chemical composition may have different morphology depending on several variations, such as, source, generation method, and the age of particles. An approach to consider irregular or non-spherical particles is to use the so-called dynamic shape factor. The dynamic shape factor is defined as the ratio of the drag force on a particle to that on the particle's volume equivalent sphere $(13,15)$. The value of the dynamic shape factor is 1 if the particle is spherical and increases with the increase of the aspect ratio. Several measurement and prediction show that the dynamic shape factor varies with 
the physical properties and shape of particles (13,19-22). A recently developed fractal concept is also a promising approach to describe the morphological effect and has been applied to the analysis of aerosol dynamics $(23,24)$. For non-spherical particles the distribution function can be expressed as

$$
n\left(m_{1}, m_{2}, \ldots, m_{s}, \chi, t\right)
$$

where $\chi$ accounts for the morphological effect. Though the morphological effect may be important to aerosol dynamics, however, usually it has no direct relation with the chemical composition variation effect. Since our main interest is on mixed particles and their effects on aerosol dynamics, the morphological effect will not be treated here and particles are assumed to be spherical.

Note that the simpler definitions based on the one-dimensional domain are in fact the moments of the multidimensional distribution. For example, let the number distribution of the spherical particles be $n\left(m_{1}, m_{2}, \ldots, m_{s-1}, M, t\right)$. Then the mass concentration of the $i$-th component in the $l$-th section in the component mass concentration-size distribution, $q_{i, l}$, can be represented as

$$
q_{i, l}=\int_{M_{l-1}}^{M_{l}} \int_{0}^{M} \ldots \int_{0}^{M} m_{i} n\left(m_{1}, m_{2}, \ldots, m_{s-1}, M, t\right) d m_{1} d m_{2} \ldots d m_{s-1} d M
$$

where $M_{l-1}$ and $M_{l}$ are the lower and the upper limits of the $l$-th section, respectively. Also the number distribution on the one-dimensional domain, $n(M, t)$, can be given as

$$
n(M, t)=\int_{0}^{\infty} \ldots \int_{0}^{\infty} n\left(m_{1}, m_{2}, \ldots, m_{s-1}, M, t\right) d m_{1} d m_{2} \ldots d m_{s-1} .
$$

Also, the total number concentration, $N$, is expressed

$$
N=\int_{0}^{\infty} \ldots \int_{0}^{\infty} n\left(m_{1}, m_{2}, \ldots, m_{s-1}, M, t\right) d m_{1} d m_{2} \ldots d m_{s-1} d M
$$

Therefore, the simpler definitions can be regarded as the integrated properties of the multidimensional definition. Similarly, average, bulk, or apparent properties can be derived from the number-chemical composition distribution. For example, the 
average density of particles in a certain size range, $\rho_{l}$, can be calculated as

$$
\rho_{l}=\left(\sum_{i=1}^{s} \int_{0}^{\infty} \ldots \int_{0}^{\infty} \rho_{i} n\left(m_{1}, m_{2}, \ldots, m_{s}, t\right) d m_{1} d m_{2} \ldots d m_{s}\right) / N
$$

where $\rho_{i}$ is the density of $i$-th component.

One disadvantage of this definition is that it requires very large experimental and computational burdens. A related problem arises if chemical compositions are used as the independent variables since experimental data are seldom comprehensive enough to be described by it. On the contrary, integrated properties of an aerosol on a small size range, such as extinction coefficient or density, are easier to measure than the individual particles' chemical composition. Thus, an interesting and important problem is whether the easily measurable average properties can be used as the independent variables instead of chemical composition.

Let $n^{\prime}\left(z_{1}, z_{2}, \ldots, z_{s-1}, M, t\right)$ be the number distribution based on the multidimensional domain where $z_{i}$ is the $i$-th average property. Then the relation between $n^{\prime}\left(z_{1}, z_{2}, \ldots, z_{s-1}, M, t\right)$ and $n\left(m_{1}, m_{2}, \ldots, m_{s}, t\right)$ is

$$
\begin{gathered}
n\left(m_{1}, m_{2}, \ldots, m_{s}, t\right)=J n^{\prime}\left(z_{1}, z_{2}, \ldots, z_{s-1}, M, t\right) \\
J=\frac{\partial\left(z_{1}, z_{2}, \ldots, z_{s-1}, M\right)}{\partial\left(m_{1}, m_{2}, \ldots, m_{s}\right)}
\end{gathered}
$$

where $J$ is the Jacobian (determinant).

From this relation, two problems emerge; (1) whether the correlation between $z_{i}$ and $m_{j}$ can be obtained; and (2) whether the relation of Eq. (11) is unique if the correlation exists, in other words, whether the relation is well-posed.

Average properties are integral properties as Jaenicke (25) pointed out,

$$
z_{i}=\int_{0}^{\infty} \ldots \int_{0}^{\infty} w_{i}\left(m_{1}, m_{2}, \ldots, m_{s}, t\right) n\left(m_{1}, m_{2}, \ldots, m_{s}, t\right) d m_{1} d m_{2} \ldots d m_{s}
$$

where $w_{i}$ is the weighting function of the $i$-th average property, $z_{i}$. At first, the correlation between the single particle property (essentially the weighting function) and chemical composition should be obtained which may be obtained theoretically 
or/and experimentally. Specifically, each particle's chemical composition and specific property can be measured individually for a large number of particles and then the empirical correlation or parameters of a theoretical correlation can be obtained. Ouimette and Flagan (26), for example, derived the correlation between extinction coefficient and composition variation. They derived single particle extinction coefficient for several cases and correlated the theoretical analysis with the measurement data. In general, however, to obtain the necessary correlations is a formidable task. Furthermore, as Fric ander pointed out (27), the average property measurement may provide excessive information compared to the individual particle analysis.

Even if the correlation between single particle property and chemical compositi.... is obtained, another difficulty arises in the integration in Eq. (13) since the equatw: contains the number distribution based on the chemical composition domain whin in is to be calculated, so the system is an implicit one.

The above mentioned difficulties are related to the problem of obtaining the currelation between average property and chemical composition. Another question is whether Eq. (11) is well-posed, i.e., whether for a given number-average property distribution there is a unique number-chemical composition distribution.

In general, Eq. (11) is not well-posed and there may be no number-chemical composition distribution or multiple distributions that satisfies it. If there are multiple distributions, to obtain the appropriate one is another problem that is called the inversion problem. Some inversion schemes have been proposed and used in aerosol classification $(28,29)$. The problem of correlating relative easily measurable integral properties with the number-chemical composition distribution has not been studied extensively and further research is warranted.

\subsection{Mixed Particles}

In many cases, we are interested in the difference of one property among particles. Also many measurement methods are limited to reveal only one property difference. Hygroscopicity in a cloud chamber and optical properties in an OPC are good exam- 
ples. For these cases, the concept of mixed particles is a useful definition.

After Winkler (12) introduced the concept of internally and externally mixed particles, a few formal definitions of them have been proposed. Winkler (12) proposed the standard deviation of the relative composition of individual particles as the degree of mixing parameter,

$$
\sigma=\frac{1}{n-1} \sum_{i=1}^{n}\left(x-x_{i}\right)^{2}
$$

where $\sigma$ is the standard deviation of each particle's property, $x_{i}$, from its mean value, $x$, and $n$ is the number of particles. This definition is rigorous and considers individual particles' composition variations. But the measurement of individual particles is a formidable task. Furthermore, the standard deviation parameter does not provide a specific value for the external mixture and the parameter can have the same value for particles of a different degree of mixing (25). Therefore, this parameter does not clearly identify the degree of mixing.

Jaenicke (25) proposed degree of mixing parameters based on the average volume and number mixing ratios. The internal mixing ratio, $M_{I}$, is defined as

$$
M_{I}=\frac{M_{n}-M_{v}}{1-M_{v}}=\frac{n_{S} V_{T}-n_{T} V_{S}}{n_{T} V_{T}-n_{T} V_{S}}
$$

where $n_{S}$ and $V_{S}$ are the number and volume concentrations of particles containing the substance of interest, $S$, respectively; $n_{T}$ and $V_{T}$ are the total number and volume concentrations of the aerosol, respectively. $M_{n}=\frac{n_{S}}{n_{T}}$ and $M_{v}=\frac{V_{S}}{V_{T}}$ are the number and volume mixing ratios, respectively. Also the external mixing ratio, $M_{E}$, is

$$
M_{E}=1-M_{I}
$$

While these mixing ratios can be obtained relatively easily, the definition can have the same value for particles of different distributions. For example, consider ten particles in which $50 \%$ of the volume concentration is the substance of interest, S, i.e., $M_{v}=0.5$. If $\mathrm{S}$ is uniformly distributed among the particles, $M_{n}=1$ and therefore, $M_{I}=1$. But the value of that is still 1 in the case of two particles consisting of pure $\mathrm{S}$ and the remaining $S$ being evenly distributed over the other eight particles since still 
$M_{n}=1$. In other words, this definition only considers the presence of the substance of interest but does not consider whether the particles are pure or a mixture of several compounds.

The problem of these definitions is that variation of the possible distribution variations are too broad to be adequately specified. As shown in Figure 3, there may be a large number of possible distributions between two extremes with the constraint of the same mean mass (or volume) fraction.

Internally mixed particles are defined as the particles in which the fraction of the substance of interest is the same as the average fraction of the total particles, or

$$
n\left(m_{S}, M-m_{S}, t\right) \neq 0 \quad \text { if } m_{S}=0 \text { or } m_{S}=M \text {. }
$$

Externally mixed particles are defined as the particles consisting of either the pure substance of interest or other substances, or

$$
n\left(m_{S}, M-m_{S}, t\right) \neq 0 \quad \text { if } \frac{m_{S}}{M}=\left(\frac{\overline{m_{S}}}{M}\right) .
$$

But to define arbitrarily mixed particles, it is desirable to consider more limited situations. Then the degree of external mixing, $D_{E}$, may be defined

$$
D_{E}=\frac{n_{E}}{n_{T}}
$$

and the degree of external mixing of the substance, $S$, is

$$
D_{E, S}=\frac{n_{S}}{n_{T}}
$$

where $n_{T}, n_{E}$, and $n_{S}$ are the total, externally mixed and pure $S$ number concentrations of the aerosol, respectively. This definition can be obtained from relatively easily measurable quantities and can uniquely determine the degree of external mixing. The degree of internal mixing is not obtained from this definition, but as mentioned earlier one parameter cannot describe all possible variations. Therefore, this simple definition is sufficient to describe present measurement data and techniques. 


\section{EXPERIMENTAL METHODS}

There are several ways of investigating the number-chemical composition distribution. Among them, the most direct and convincing way of showing the existence of the distribution is to measure chemical composition of individual particles. Several chemical analysis techniques have been applied for this purpose.

The electron microscope has been used for measuring individual particles. The scanning electron microscope (SEM) with an energy dispersive spectrometer (EDS) $(30,31)$ and its refined version, the electron probe X-ray microanalysis (EPXMA) $(32,33)$ has been used to measure chemical compositions of a large number (usually several thousand) of atmospheric aerosol particles. The EPXMA provides quantitative elemental analysis for above a limiting atomic number with relatively short time (compared to previous electron microscopy analyses) by using automated data reduction scanning technique. Also the EPXMA measures morphology, size and shape. of each particle. But the required time for analysis is still long and usually this method does not provide information about molecular species. Furthermore, it has the limitation in detecting certain elements.

Mass spectrometry, also, has been used for the analysis of individual particles. Sinha et al. (34) developed the particle analysis by mass spectrometry (PAMS) in which micron size monodisperse individual particles are volitized by impaction on a hot rhenium V-type filament $\left(300\right.$ to $\left.1400^{\circ} \mathrm{C}\right)$ and ionized, and the resulting vapor is measured by a quadrupole mass spectrometer in real-time. This method was applied to measure sodium mass concentration quantitatively $(35,36)$. The laser microprobe mass analysis (LAMMA) $(32,37)$ is another instrument used for single particle chemical analysis in which individual particles are excited or vaporized and partially ionized by the laser power and then analyzed by a mass spectrometer. Similarly, in the laser spark spectroscopy or laser-induced breakdown spectroscopy (LIBS) (38) individual particles are vaporized by a pulsed laser and atomic emission spectra are measured.

Generally, these method are fast, have a lower detection limit than the EPXMA, and can identify molecular species in addition to the elemental analysis. Further- 
more, these can be used in situ. But particles are destroyed to produced spectra and cannot be used furthermore. Also due to the difficulty in calibration and the poor reproducibility, these are used primarily for the qualitative analysis.

In general, single particle analysis techniques are, though they have limited applications and are not in the state of routine analysis, promising and more applications are expected with the development and improvement of techniques.

A class of methods exploits the property differences of particles to measure property variation. In these methods, particles are classified by an instrument according to their size and then further classified according to the property difference. So these can be regarded as a partial resolution of the number-property distribution. Frequently used properties are the commonly measured ones such as hygroscopicity. For example, Okada (39) examined the fraction of hygroscopic particles and that of the watersoluble material in individual mixed particles by observing the particle size change before and after the dialysis by an electron microscope. Covert and Heintzenberg (40) used two cascade impactors with different relative humidities (RHs) to collect urban atmospheric aerosol samples of which chemical compositions were analyzed. By measuring the shift of particle mass and chemical compounds by the hygroscopic growth at high RH, the degree of external mixing of water soluble (sulfur and other compounds) vs. water insoluble (soot) materials in the aerosol was measured. Harrison (41) used a thermal diffusion cloud chamber in series with a dichomotous separator to measure the cloud condensation nuclei ( $\mathrm{CCN})$ fraction of atmospheric aerosol.

These experiments measured the size difference between particles after humidification and those before (or without) humidification, sometimes in conjunction with chemical analysis. In these experiments, the fraction of externally mixed nonhygroscopic particles is obtained. But the aerosol samples used were not monodisperse but ranged over a relatively large size. Therefore, the intermingling of the effect of particle size with that of property variation for one size complicated the analysis of experimental data.

It is desirable to use monodisperse (or practically very narrow size range) particles 
for the experiments. A few monodisperse aerosol generators are now available (42), among them, the differential mobility analyzer (DMA) is well suited for generating monodisperse particles smaller than $0.1 \mu \mathrm{m}$ diameter. The DMA, developed at the University of Minnesota (43-46), screens particles according to their electric mobility very accurately. It measures the number-size distribution by measuring aerosol concentration $v s$. electric mobility and also generates monodisperse aerosol by screening particles of only one electric mobility from polydisperse feed particles. Application of the DMA has been further enhanced by improvements of the DMA technology. The scanning electrical mobility spectrometer (SEMS) (47) reduces the required measurement time by an order of magnitude over that of the conventional DMA by using a time-varying electric field instead of the discrete step changing electric field. The electromobility spectrometer (48) based on the DMA has been developed to measure particles as small as $1 \mathrm{~nm}$ diameter by using a very sensitive electrometer.

The relation between electric mobility and particle size (usually diameter) is not unique since particles of different sizes can have the same mobility through multiple charging,

$$
Z_{p}=\frac{i C_{d} e}{3 \pi \mu D_{p i}}
$$

where $Z_{p}$ is the electric mobility, $C_{d}$ is the slip correction factor, $e$ is the charge of an electron, $1.6021 \times 10^{-19} \mathrm{C}, \mu$ is the gas viscosity, and $D_{p i}$ is the diameter of a particle having $i$ elementary charges. Therefore, for a fixed value of electric mobility, there is a spectrum of corresponding particle diameters. For the measurement of the numbersize distribution many inversion procedures have been developed that account for the multiple charging effect $(28,29,46,49-52)$, but for monodisperse aerosol generation the presence of multiply charged particles is not permitted. See, for example, Reischl (53) for the effect of several input conditions of the DMA on output variation. For bipolar charging, which is used in the conventional DMA, particles of sizes below $0.1 \mu \mathrm{m}$ can be considered as singly charged. Above that size, the fraction of multiply charged particles increases dramatically according to either the Boltzmann or Fuchs charging 
probability distribution $(5,54,55)$. Studies to increase the singly charged particle size are in progress (56).

Instruments that measure a size and/or a number-size distribution of an aerosol can be classified according to the operating principle of the instrument as shown in Table II. Pui and Liu (42) reviewed instruments for measuring the number-size distribution and for monodisperse particle generation. Monodisperse particles screened by the DMA can be classified and/or separated further according to their other property variation by either another number-size measurement instrument of which the operating principle is based on that property variation of the particles or the combination of an aerosol condition changer and a number-size distribution measurement instrument.

For example, particles of the same size can have an optical property distribution that can be resolved by the optical particle counter (OPC). Covert et al. (60) measured optical property variation of monodisperse atmospheric particles screened $\mathrm{b}$. the DMA and observed on some occasions that the monodisperse particles developed two distinct size peaks at the OPC. They concluded that the aerosol was externally mixed and consisted of soot (light absorbing) and inorganic materials, sulfate and nitrate compounds (light non-absorbing). If two components are internally mixed the OPC should register a single size, while if two components are externally mixed, the OPC would give two particle sizes due to the difference of refractive indices between the soot and inorganic materials. Hering and McMurry (61) also used the DMA-single particle optical counter and determined the optical counter response to monodisperse atmospheric aerosols. They found that the response sometimes differed from those of the several different calibration aerosols of the same size. Also, on several occasions, monodisperse atmospheric aerosols produced two distinct optical diameters that indicated some degree of external mixing of chemical compounds of different optical properties.

Another possible combination of instruments is the DMA-inertial instrument such as the cascade impactor or the aerodynamic article sizer (APS). These instruments 
separate particles according to their inertia difference, therefore, monodisperse particles screened by the DMA but having different densities should show a number-density distribution. Cascade impactors $(62,63)$ or the APS (64-66) are common instruments and this combination can be easily utilized. A DMA-APS system was used to measure the number-size-electric charge distribution (67). A DMA-hypersonic impactor system was also used (68) to study the performance of the supersonic impactor.

A problem associated with these combinations is that the ranges of the size resolution of individual instruments may not be compatible. For example, the DMA usually has an upper size limit of $0.1 \mu \mathrm{m}$ for monodisperse particle generation while that of the conventional cascade impactors have a lower size limit of $0.3 \mu \mathrm{m}$ and that of the APS a lower size limit of $0.8 \mu \mathrm{m}$. This overlap problem has been partially solved by several approaches; a low pressure impactor (69), use of small size nozzles (70), and a hypersonic impactor (68).

Other combinations are also possible, for example, the DMA-diffusion battery provided that the ranges of particle size resolved for each instrument are mutually compatible. While these methods have succeeded in demonstrating the existence of property variations and in measuring the degree of mixing, they do not utilize the property distribution, for example, do not separate particles.

The tandem differential mobility analyzer (TDMA) differs from the previously described instruments since in the TDMA the property difference induces particles size and/or concentration change(s) at the aerosol conditioner between the two DMAs and the changes are measured at the second DMA (DMA2). A schematic diagram of the TDMA system is given in Figure 4; polydisperse feed partirles are charged in the charger and screened by the DMA1 according to their electric mobility. The screened monodisperse particles are treated in the aerosol conditioner in which the size and/or concentration of particles are intentionally altered and then measured by the DMA2 and a particle concentration sensor. Several applications of the TDMA have been reported: evaporation rates, vapor pressures (71), and surface free energy (72). The TDMA is ideally suited for studying property variation of particles of the 
same size since one can control the processes occurring in the aerosol conditioner in which particle size and/or concentration changes occur. Liu et al. (73) and McMurry and Stolzenburg (74) demonstrated that particles of the same size can be separated according to their hygroscopicity difference. Using a gas-phase diffusion adsorber and a humidifier as the aerosol conditioner Hansson et al. (75) studied the hygroscopicity change due to organic coatings on hygroscopic aerosols. Similar measurements were reported in the DMA-multistep condensation nucleus counter (CNC) in which the multistep CNC acted as the combination of the conditioner and the DMA2 in the TDMA system (76).

The interpretation of TDMA data depends on the processes occurring in the conditioner: (1) there may be size changes, shift of the peak and/or generation of multiple peaks depending on the mixedness of particles and the processes; (2) there may be concentration changes since some processes are not number-conserving; and (3) there may be multiple processes occurring in the conditioner, though, in many cases by a careful manipulation of conditions in the conditioner all but one process can be eliminated. McMurry and coworkers $(77,78)$ proposed a numerical routine to invert the TDMA measurement data that is based on the conventional DMA analysis (44) with approximations for the two DMAs and a geometrical growth model with dispersion with approximations for the conditioner. The routine requires three parameters for a distribution peak and the values of these are obtained by a least squares fitting procedure. While this method can fit the size and concentration changes, it does not model the fundamental aerosol dynamic processes occurring in the conditioner.

Ideally, the aerosol dynamic processes in the conditioner should be fundamentally modeled to study the changes in the conditioner. This can be accomplished by using a numerical routine that solves the multicomponent aerosol general dynamic equation (GDE) that governs the fundamental aerosol dynamic processes in the homogeneous gas phase. This approach is the subject of Chapter IV of this thesis.

With improvements in instrumentation and in aerosol modeling for interpreting TDMA data, the application of the TDMA to property variation studies should in- 
crease. Some promising applications are: (1) to measure the fraction and the degree of mixing by selective chemical reaction; (2) to measure the effect of the degree of surface exposure on aerosol property variation. Particles of the same size and same composition may have different properties depending on which compound is dominant on the particle surface; (3) to study the effects of multiple processes occurring simultaneously.

\section{NUMERICAL SIMULATION}

Dynamics of a spatially homogeneous multicomponent aerosol is governed by the so-called multicomponent aerosol general dynamic equation (GDE) with chemical composition as the independent variables,

$$
\begin{aligned}
& \frac{\partial n(\mathbf{m}, t)}{\partial t}+\sum_{i=1}^{s} \frac{\partial}{\partial m_{i}}\left[I_{i}(\mathbf{m}, t) n(\mathbf{m}, t)\right] \\
& =\frac{1}{2} \int_{0}^{m_{1}} \cdots \int_{0}^{m_{s}} \beta(U, M-U) n(\mathbf{m}-\mathbf{u}, t) n(\mathbf{u}, t) d \mathbf{u} \\
& -n(\mathbf{m}, t) \int_{0}^{\infty} \cdots \int_{0}^{\infty} \beta(U, M) n(\mathbf{u}, t) d \mathbf{u} \\
& +R[\mathbf{m}, t, n(\mathbf{m}, t)]+S[\mathbf{m}, t, n(\mathbf{m}, t)]
\end{aligned}
$$

where $m_{i}$ is the mass of the $i$-th component in a particle, and $\mathbf{m}$ is a vector of compositions $\left(m_{1}, \ldots, m_{s}\right)$, where $s$ is the total number of components. $n(\mathbf{m}, t) d \mathbf{m}$ is the number of particles having mass of component $i$, in the range $\left[m_{i}, m_{i}+d m_{i}\right]$ at time $t . I_{i}$ is the time rate change of the mass concentration of the $i$-th component from condensation/evaporation processes. $\beta(U, M)=\beta(M, U)$ is the binary coagulation coefficient where $U$ and $M$ are particle sizes given by $M=\sum_{j=1}^{s} m_{j} . R$ and $S$ are removal and source terms, respectively. The initial condition is

$$
n(\mathbf{m}, 0)=n_{o}(\mathbf{m})
$$

The boundary condition specifies no particles at the critical cluster size

$$
n\left(\mathrm{~m}^{*}, t\right)=0
$$


where $\mathbf{m}^{*}$ is the size of critical cluster.

Simulation of multicomponent aerosol dynamics presents serious computational challenges (79). Condensation/evaporation, coagulation, nucleation, and deposition have inherently different mathematical and physical characteristics. For example, condensation/evaporation are number conserving processes, while coagulation is a mass conserving process. And, condensation/evaporation and nucleation are compositiondependent processes, while coagulation and deposition are the composition-independent processes (neglecting shape and sticking effects). A numerical approach that works well for simulating one particular process may not be suitable for simulating other processes. Also the computational burden to simulate a multicomponent system is potentially very large since in addition to the multidimensionality the time step is limited by the shortest characteristic time among those of the individual processes Finally, the ideal way to test the performance of a numerical routine is to compare the numerical solution with the analytical solution but analytical solutions for multicomponent aerosol dynamics are available only in very limited cases $(80,81)$.

Several numerical routines have been developed to simulate aerosol dynamics. These approaches can be classified according to whether the aerosol consists of single or multiple components and whether the computational domain is one-dimensional or multidimensional. Table III summarizes some of the available numerical codes based on these criteria.

A numerical routine for multicomponent aerosol dynamics should satisfy the following properties:

1. The numerical schemes adopted in the routine guarantee the distribution be positive definite and the total number concentration be conserved.

2. The dynamics of arbitrarily mixed particles can be simulated.

3. Simultaneous processes in addition to single process can be simulated. 
4. The numerical schemes used are accurate.

5. The numerical schemes used are efficient.

The first three properties arise from the need for physical consistency between the real system and the simulated system and the last two properties are desirable from a numerical analysis point of view. Unfortunately, no single numerical method satisfies all the above properties. For condensation/evaporation, for example, the finite element methods (FEMs) are accurate and efficient but do not satisfy the property 1 , while the upwind difference methods satisfy property 1 but are not as accurate as the FEMs. On the other hand, numerical schemes based on a Lagrangian approach in which grids move with the movement of particles satisfy all properties except the property 3 (85). Finally, Gaussian quadrature for coagulation satisfies all properties except the final one; it takes a large computing time.

A few numerical methods have been developed to simulate multicomponent aerosol dynamics. The first and arguably the most widely used program is MAEROS (Multicomponent AEROSol) $(86,87)$ which is based on the multicomponent sectional method (17). As noted earlier, in the sectional method, the one-dimensional size computational domain is divided into a finite number of sections (or elements) and in each section the moment of number distribution (number, surface area, mass or volume concentration, in MAEROS the total mass concentration is calculated) is assumed to be uniform (88). Also, in the multicomponent case, the mean mass fraction of each component in a section is assumed to be uniform (17). The sectional approach has been successfully applied to several areas of aerosol dynamics research: simulation of multicomponent aerosol dynamics in plumes $(89,90)$, urban photochemical aerosol $(91,92)$, simulation of particles in a nuclear reactor $(86)$, simulation of indoor aerosol dynamics (93), and the discrete-sectional method for simulating the aerosol evolution associated fast chemical reactions (94).

Based on the two approximations, in MAEROS, the total and components mass concentration balances in a section are derived. The model reduces to a system of 
$(s \times l)$ ordinary differential equations where $s$ is the number of components and $l$ is the number of sections. Coagulation is treated by considering all possible combinations of the two body agglomeration which results in double integrations to calculate coagulation coefficients. Condensation/evaporation is treated with a second-order approximation for the concentration distribution.

MAEROS has several advantages: (1) it has an sxcellent User's manual $(86,87)$; (2) it has been widely tested; (3) it guarantees satisfaction of certain fundamental physical properties, such as the distribution being positive definite and the total mass concentration being conserved; (4) it can simulate simultaneous processes with different mechanisms; (5) it is highly accurate for simulating coagulation; and (6) it is efficient since the mean mass fractions are calculated on the one-dimensional domain.

But in MAEROS, only one component, water, can condense. For cases where water is the only condensible component, for example, hygroscopic growth of aerosol particles in the air, this restriction poses no serious problem. But, in general, there are several condensible components in the gas phase. One notable example is the atmospheric aerosols where condensation of several organic and inorganic compounds occurs. In this case, this restriction imposes a serious limitation on the application of MAEROS. Also, the accuracy of the condensation/evaporation simulation scheme is low. While the assumption that the mean mass fraction of each component in a section is uniform makes it possible to simulate the average components' mass concentration on the one-dimensional computational domain, composition variation at one particle size is not calculated. In other words, a system of arbitrarily mixed aerosol particles cannot be simulated by MAEROS.

In ESMAP ( Expanded Sectional Multicomponent Aerosol Package) $(95,96)$ homogeneous nucleation and a new condensation/evaporation simulation scheme, which has a number concentration conservation constraint, were added to MAEROS in addition to several minor modifications. The basic structure of ESMAP is the same as that of MAEROS, it has generally the same properties as MAEROS.

Pilinis et al. $(91,92)$ extended MAEROS and developed a routine in which multi- 
ple components can condense and/or evaporate for simulating atmospheric aerosols. To do that, however, all particles in one section are assumed to have the same compositions. This is more of a severe assumption than that in MAEROS in which particles can have composition variation even though that is not calculated.

The same problem arises in a variation of the sectional representation by Pilinis (97) in which the component mass distributions are calculated instead of the component mass concentrations. Furthermore, the numerical method used, the Galerkin method, is known to be unstable for simulating aerosol condensation/evaporation (98).

Oron and Seinfeld $(99,100)$ developed a two-dimensional sectional code to simulate dynamics of charged aerosols and ions, in which a two-dimensional, size-electric charge, computational domain is used to simulate the dynamic behavior of charged aerosols and ions which undergo coagulation, electrostatic dispersion, ion recombination, and ion and particle production and loss. Note that this routine can give insights in conducting and understanding experiments that measure and utilize the numbersize-electric charge distribution, see for example, Emets et al. (67). The resulting governing equation contains coefficients each of which requires double summation of double integrals. The number of integrations increases by two for each increase in the dimension of the computational domain (if the independent variable is a discrete one, like the charge, two summations).

The multicomponent sectional method (17) used an Eulerian approach. In this approach, grids or mashes are fixed and numerical errors (numerical diffusion and dispersion errors) occurring during the discretization of the condensation/evaporation term cannot be eliminated. Numerical schemes using a Lagrangian approach in which grids move along characteristics of condensation/evaporation processes may be free from the numerical errors associated with the discretization of the condensation/evaporation term (85). To cope with the problems of numerical routines using the multicomponent sectional method, the moving sectional method was developed $(85,101)$ and implemented to MGA (Moving Grid Analysis) (102). In the moving 
sectional method, the assumptions of the original fixed grid sectional method were used but now each section's boundaries are permitted to move along the characteristics of condensation/evaporation, i.e., the Lagrangian approach is used. By doing so, the accuracy of the condensation/evaporation simulation is greatly enhanced. Also, by allowing sections to move independently, there may be overlapping of sections in the same size range and, therefore, aerosol particles of the same size but different mean mass fractions can be simulated. Therefore, the moving sectional method can simulate the dynamics of arbitrarily mixed aerosol particles though it still keeps the assumption of uniform mean mass fractions. As in MAEROS and ESMAP, only water can condense or evaporate in MGA though in an extended moving sectional method (85) multiple components can condensate or evaporate. But a more serious problem arises because of the use of the Lagrangian approach. Since sections can move independently holes may develop in the size computational domain. MGA is suitable for pure condensation/evaporation processes but not suitable for simultaneous processes, such as coagulation.

Tsang and Rao (103) developed a numerical scheme (MULFEMM) based on the moving finite element method (FEM) for simulating condensation of a two-component aerosol. This method is an extension of the algorithm by Varoğlu and Liam Finn (104) applied to the simulation of single component aerosol dynamics (98), in which elements move along the characteristics of the system. Simulation of evaporation or mixed condensation/evaporation is difficult in this method even in the single component case due to the excessive loss of elements (98). Thus, MULFEMM is suitable for simulation only of pure condensation. Also, in this method a positive definite distribution and conservation of number concentration are not guaranteed.

The deformation of grids or elements is a common problem in numerical schemes using the Lagrangian approach. Among several approaches to address this difficulty, one approach is to remap the moving grids onto a fixed grid after each time step. Even with a high-order accuracy interpolation scheme, however, the positive definite and the conservation properties may not be maintained. On the other hand, with the 
constraint of number conservation, the remapping introduces new numerical errors and the overall accuracy deteriorates. An alternative approach is to introduce new grids whenever these are needed. If condensation/evaporation rates are not very fast then the required number of new grids may be small since the existing grids cover most of the computational domain. In general, a numerical scheme using the Lagrangian approach is ideal for pure condensation/evaporation processes but not suitable for simultaneous processes.

Chapter III describes the development of a numerical routine called AMPS (Arbitrarily Mixed Particles Simulator) that solves the multicomponent aerosol general dynamic equation directly without any assumption about the nature of the distribution (79). The main objective of this routine is to simulate simultaneous process occurring in the multicomponent aerosol system and thus study the distributed chemical composition effect on aerosol dynamics. To simulate simultaneous processes, we adopt the Eulerian approach. The processes and mechanisms treated are summarized in Table IV. Note that two numerical schemes are provided to simulate condensation/evaporation processes. This is due to the complementary nature of two schemes. The repeated upwind difference method (105-107) keeps the distribution positive definite and guarantees number conservation but is only second-order accurate and is poor in retaining sharp peaks, while the Taylor-Galerkin method (108-110) is highly accurate and retains the sharp peak of the distribution but cannot keep the distribution positive definite and cannot guarantee number conservation.

Comparison of numerical routine for multicomponent aerosol simulation for some of the desirable properties is summarized in Table V.

There has been considerable controversy about the accuracy of the repeated upwind difference method in particular and the choice of numerical schemes for condensation/evaporation in general $(103,111,112)$. While the accuracy of a numerical scheme is important, other properties, especially those related to the physical system, should also be considered. It should be recognized that at present no available advection scheme is fully satisfactory for simulation of aerosol condensation/evaporation 
and a numerical scheme should be chosen that is best suited for the purpose of the particular application.

\section{THE REMAINDER OF THIS THESIS}

The remainder of this thesis is as follow: In Chapter II, the extended moving sectional method is presented in which multiple components can condense and/or evaporate. In Chapter III, a numerical scheme for simulating multicomponent aerosol dynamics that can simulate arbitrarily mixed particles is presented. In Chapter IV. the numerical scheme is applied to simulate a TDMA experimental result by Liu \& al. (73). In Chapter V, conclusions are presented.

And in Appendix I, a review on the numerical techniques for urban airshed model: is presented and in Appendix II, a user's manual for the numerical scheme described in Chapter III is presented. 


\section{REFERENCES}

(1) Heintzenberg, J.; Covert, D.S. J. Atmos. Chem. 1990, 10, 383-397.

(2) Kneip, T.J.; Lioy, P.J. Ed. Aerosols: Anthropogenic and Natural, Sources and Transport; The New York Academy of Science: New York, 1980.

(3) Junge, C. Air Chemistry and Radioactivity; Academic Press: New york, 1963.

(4) Flagan, R.C.; Seinfeld, J.H. Fundamentals of Air Pollution Engineering; Prentice Hall: Englewood Cliffs, 1988.

(5) Whitby, K.T. In Fine Particles; pp 581-624, B.Y.H. Liu Ed.; Academic Press: New York, 1976.

(6) Mathai, C.V.; Stonefield, D.H. Ed. PM-10: Implementation of Standart: APCA: Pittsburgh, 1988.

(7) U.S. Environment Protection Agency Federal Register 1987, 52, 24634.

(8) Vincent, J.H. Aerosol Sampling: Science and Practice; John Wiley \& S.,n: Chichester, 1989.

(9) Seinfeld, J.H. Atmospheric Chemistry and Physics of Air Pollution; John Wiley \& Sons: New York, 1986.

(10) Air Sampling Instruments for Evaluation of Atmospheric Contaminants 6th Ed.; American Conference on Governmental Industrial Hygienists: Cincinnati, 1983.

(11) Hidy, G.M. Aerosols: Research, Risk Assessment and Control Strategies pp1941, Lee, S.D; Schneider, T.; Grant, L.D.; Verkerk, P.J. Ed.; Lewis Publishers, Inc.: Chelsea, 1986.

(12) Winkler, P. J. Aerosol Sci. 1973, 4, 373-387.

(13) Fuchs, N.A. The Mechanics of Aerosols; Pergamon Press: Oxford, 1964.

(14) Friedlander, S.K. Smoke, Dust and Haze: Fundamentals of Aerosol Behavior, John Wiley \& Sons: New York, 1977.

(15) Hinds, W.C. Aerosol Technology: Properties, Behavior, and Measurement of Airborne Particles; John Wiley \& Sons: New York, 1982.

(16) The Education Committee of the American Association of Aerosol Research Aerosol Sci. Technol. 1991, 14, 1-4. 
(17) Gelbard, F.; Seinfeld, J.H. J. Colloid Interface Sci. 1980, 78, 485-501.

(18) Friedlander, S.K. J. Aerosol Sci. 1970, 1, 295-307.

(19) Cheng, Y.-S.; Yeh, H.-C.; Allen, M.D. Aerosol Sci. Technol. 1988, 8, 109-123.

(20) Kops, J; Dibbets, G.; Hermans, L.; Van de Vate, J.F. J. Aerosol Sci. 1975, 6, 329-333.

(21) Colbeck, I. J. Aerosol Sci. 1990, 21, s43-s46.

(22) Brockmann, J.E.; Rader, D.J. Aerosol Sci. Technol. 1990, 13, 162-172.

(23) Clearly, T.; Samson, R.; Gentry, J.W. Aerosol Sci. Technol. 1990, 12, 518-525.

(24) Kütz, S; Schmidt-Ott, A. J. Aerosol Sci. 1990, 21, s47-s50.

(25) Jaenicke, R. Atmos. Environ. 1978, 12, 161-169.

(26) Ouimette, J.R.; Flagan, R.C.. Atmos. Environ. 1982, 16, 2405-2419.

(27) Friedlander, S.K. J. Aerosol Sci. 1971, 2, 331-340.

(28) Twomey, S. J. Compute. Phys. 1975, 18, 188-200.

(29) Wolfenbarger, J.K.; Seinfeld, J.H. J. Aerosol Sci. 1990, 21, 227-247.

(30) Post, J.E.; Buseck, P.R. Environ. Sci. Technol. 1984, 18, 35-42.

(31) Saucy, D.A.; Anderson, J.R.; Buseck, P.R. Atmos. Environ. 1987, 21, 16491657.

(32) Bruynseels, F.; Storms, H.; Van Grieken, R.; Van der Auwera, L. Atmos. Environ. 1988, 22, 2593-2602.

(33) Artaxo, P.; Maenhaut, W.; Storms, H.; Van Grieken, R. J. Geophys. Res. 1990, 95, 16971-16985.

(34) Sinha, M.P.; Giffin, C.E.; Norris, D.D.; Estes, T.J.; Vilker, V.L.; Friedlander, S.K. J. Colloid Interface Sci. 1982, 87, 140-153.

(35) Sinha, M.P.; Friedlander, S.K. Anal. Chem. 1985, 57, 1880-1883.

(36) Giggy, C.L.; Friedlander, S.K.; Sinha, M.P. Atmos. Environ. 1989, 23, 2223-2229. 
(37) Denoyer, E.; Natusch, D.F.S.; Surkyn, P.; Adams, F.C. Environ. Sci. Technol. 1983, 17, 457-462.

(38) Ottesen, D.K.; Wang, J.C.F.; Radziemski, L.J. Appl. Spectro. 1989, 43, 967-976.

(39) Okada, K. J. Met. Soc. Japan 1983, 61, 727-736.

(40) Covert, D.S.; Heintzenberg, J. Sci. Total Environ. 1984, 36, 347-352.

(41) Harrison, L. J. Clim. Appl. Met. 1985, 24, 312-321.

(42) Pui, D,Y.H.; Liu, B.Y.H. Physica Scripta 1988, 37, 252-269.

(43) Liu, B.Y.H.; Pui, D.Y.H. J. Colloid Interface Sci. 1974, 47, 155-171.

(44) Knutson, E.O.; Whitby, K.T. J. Aerosol Sci. 1975, 6, 443-451.

(45) Knutson, E.O.; Whitby, K.T. J. Aerosol Sci. 1975, 6, 453-460.

(46) Knutson, E.O. In Fine Particles; pp 740-762, B.Y.H. Liu ed.; Academic Press: New York, 1976.

(47) Wang, S.C.; Flagan, R.C. Aerosol Sci. Technol. 1990, 13, 230-240.

(48) Winklmayr, W.; Reischl, G.P.; Lindner, A.O.; Berner, A. J. Aerosol Sci. 1991, 22, 289-296.

(49) Hoppel, W.A. J. Aerosol Sci. 1978, 9, 41-54.

(50) Haaf, W. J. Aerosol Sci. 1980, 11, 189-200.

(51) Alofs, D.J.; Balakumar, P. J. Aerosol Sci. 1982, 13, 513-527.

(52) Reischl, G.P. Aerosol Sci. Technol. 1991, 14, 5-24.

(53) Reischl, G.P. J. Aerosol Sci. 1991, 22, 297-312.

(54) Kousaka, Y.; Okuyama, K.; Endo, Y. J. Aerosol Sci. 1981, 12, 339-348.

(55) Kousaka, Y.; Okuyama, K.; Adach, M. Aerosol Sci. Technol. 1985, 4, 209-225.

(56) Gupta, A.; McMurry, P.H. Aerosol Sci. Technol. 1989, 10, 451-462.

(57) Liu, B.Y.H.; Pui, D.Y.H. J. Aerosol Sci. 1975, 6, 249-264.

(58) Willeke, K.; Liu, B.Y.H. In Fine Particles; pp 697-729, B.Y.H. Liu Ed.; Academic Press: New York, 1976.

(59) Sinclair, D. Aerosol Sci. Technol. 1986, 5, 187-204. 
(60) Covert, D.S.; Heintzenberg, J.; Hansson, H.-C. Aerosol Sci. Technol. 1990, $12,446-456$.

(61) Hering, S.V.; McMurry, P.H. Atmos. Environ. 1991, 25A, 463-468.

(62) Marple, V.A.; Willeke, K. In Fine Particles; pp 411-446, B.Y.H. Liu Ed.; Academic Press: New York, 1976.

(63) Chuan, R.L. In Fine Particles; pp 763-777, B.Y.H. Liu Ed.; Academic Press: New York, 1976.

(64) Wilson, J.C.; Liu, B.Y.H. J. Aerosol Sci. 1980, 11, 139-150.

(65) Agarwal, J.K.; Remiarz, R.J.; Quant, F.R.; Sem, G.J. J. Aerosol Sci. 1982, $12,222-223$.

(66) Chen, B.T.; Cheng, Y.S.; Yeh, H.C. J. Aerosol Sci. 1989, 20, 1489-1492.

(67) Emets, E.P.; Kascheev, V.A.; Poluektov, P.P. J. Aerosol Sci. 1991, 22, 389-394.

(68) De la Mora, J.F.; Hering, S.V.; McMurry, P.H. J. Aerosol Sci. 1990, 21, 169-187.

(69) Hering, S.V.; Flagan, R.C.; Friedlander, S.K. Environ. Sci. Technol. 1978, $12,667-673$.

(70) Marple, V.A.; Liu, B.Y.H.; Kuhlmey, G.A. J. Aerosol Sci. 1981, 12, 333-337.

(71) Rader, D.J.; McMurry, P.H.; Smith, S. Aerosol Sci. Technol. 1987, 6, 247-260.

(72) Tao, Y.; McMurry, P.H. Environ. Sci. Technol. 1989, 23, 1519-1523.

(73) Liu, B.Y.H.; Pui, D.Y.H.; Whitby, K.T.; Kittelson, D.B.; Kousaka, Y.; McKenzie, R.L. Atmos. Environ. 1978, 12, 99-104.

(74) McMurry, P.H.; Stolzenburg, M.R. Atmos. Environ. 1989, 23, 497-507.

(75) Hansson, H.-C.; Wiedensohler, A.; Rood, M.J.; Covert, D.S. J. Aerosol Sci. 1990, 21, s241-s244.

(76) Niessner, R.; Daeumer, B.; Klockow, D. Aerosol Sci. Technol. 1990, 12, 953-963.

(77) Rader, D.J.; McMurry, P.H. J. Aerosol Sci. 1986, 17, 771-787. 
(78) Stolzenburg, M.R.; McMurry, P.H. TDMAFIT User's Manual; PTL Publication No. 653, University of Minnesota: Minneapolis, 1988.

(79) Kim, Y.P.; Seinfeld, J.H. Aerosols: Science, Industry, Health and Environment; pp .38-141, S. Masuda; K. Takahashi Ed.; Pergamon Press: Oxford, 1990.

(80) Gelbard, F.; Seinfeld, J.H. J. Colloid Interface Sci. 1978, 63, 472-479.

(81) Simons, S. Ann. Nucl. Energy 1982, 9, 473-479.

(82) Gelbard, F. AEROSOL Users Manual; NUREG/CR-1367, SAND80-0403, R7, Sandia National Laboratories, 1981.

(83) Whitby, K.T. J. Aerosol Sci. 1981, 12, 174-178.

(84) Seigneur, C.; Hudischewskyj, A.B.; Seinfeld, J.H.; Whitby, K.T.; Whitby, E.R.; Brock, J.R.; Barnes, H.R. Aerosol Sci. Technol. 1986, 5, 205-222.

(85) Kim, Y.P.; Seinfeld, J.H. J. Colloid Interface Sci. 1990, 135, 185-199.

(86) Gelbard, F. MAEROS User Manual; NUREG/CR-1391, SAND80-0822, Sandia National Laboratories, 1982.

(87) Gelbard, F. MAEROS-2 User Manual; Sandia National Laboratories, 1990.

(88) Gelbard, F.; Tambour, Y.; Seinfeld, J.H. J. Colloid Interface Sci. 1980, 76, 541-556.

(89) Bassett, M.; Gelbard, F.; Seinfeld, J.H. Atmos. Environ. 1981, 15, 23952406.

(90) Hudischewskyj, A.B.; Seigneur, C. Environ. Sci. Technol. 1989, 23, 413421.

(91) Pilinis, C.; Seinfeld, J.H.; Seigneur, C. Atmos. Environ. 1987, 21, 943-955.

(92) Pilinis, C.; Seinfeld, J.H. Atmos. Environ. 1988, 22, 1985-2001.

(93) Nazaroff, W.W.; Cass, G.R. Environ. Sci. Technol. 1989, 23, 157-166.

(94) Wu, J.J.; Flagan, R.C. J. Colloid Interface Sci. 1988, 123, 339-352.

(95) Warren, D.R.; Seinfeld, J.H. Aerosol Sci. Technol. 1985, 4, 31-43.

(96) Wang, S.H. 1990, private communication.

(97) Pilinis, C. Atmos. Environ. 1990, 24A, 1923-1928.

(98) Tsang, T.H.; Brock, J.R. Aerosol Sci. Technol. 1983, 2, 311-320. 
(99) Oron, A.; Seinfeld, J.H. J. Colloid Interface Sci. 1989, 133, 66-79.

(100) Oron, A.; Seinfeld, J.H. J. Colloid Interface Sci. 1989, 133, 80-90.

(101) Gelbard, F. Aerosol Sci. Technol. 1990, 12, 399-412.

(102) Gelbard, F. MGA User Manual; Sandia National Laboratories, 1990.

(103) Tsang, T.H.; Rao, A. Int'l J. Numer. Meth. in Fluids 1990, 10, 753-769.

(104) Varoḡlu, E; Liam Finn, W.D. J. Comp. Phys. 1980, 34, 371-389.

(105) Smolarkiewicz, P.K. Mon. Wea. Rev. 1983, 111, 479-486.

(106) Smolarkiewicz, P.K. J. Comp. Phys. 1984, 54, 325-362.

(107) Smolarkiewicz, P.K.; Clark, T.L. J. Comp. Phys. 1986, 67, 396-438.

(108) Donea, J. Int'l J. Numer. Meth. in Eng. 1984, 20, 101-119.

(109) Selmin, V.; Donea, J.; Quartrapelle, L. Compt. Meth. Appl. Mech. Lin, $1985,52,817-845$.

(110) Donea, J.; Quartapelle, L.; Selmin, V. J. Comp. Phys. 1987, 70, 463-4149.

(111) Tsang, T.H.; Rao, A. Aerosol Sci. Technol. 1988, 9, 271-277.

(112) Chock, D.P. Atmos. Environ. 1990, 24A, 1-19. 
Table I. Aerosol Moments

Based on the Number-Diameter Distribution

\begin{tabular}{cl}
$w\left(D_{p}\right)^{1}$ & \multicolumn{1}{c}{ Moment $\left(M_{w}{ }^{1}\right)$} \\
\hline 1 & Total number concentration $(N)$ \\
$\pi D_{p}^{2}$ & Total surface area concentration $(S)$ \\
$\frac{\pi}{6} D_{p}^{3}$ & Total volume concentration $(V)$ \\
$\frac{\pi}{6} D_{p}^{3} \rho$ & Total mass concentration $(M)$ \\
$\frac{D_{p}}{N}$ & Mean diameter $\left(\overline{D_{p}}\right)$ \\
$\frac{\left(D_{p}-\overline{D_{p}}\right)^{2}}{N}$ & Standard deviation $\left(\sigma^{2}\right)$ \\
\hline${ }^{1} M_{w}=\int_{0}^{\infty} w\left(D_{p}\right) n\left(D_{p}, t\right) d D_{p}$.
\end{tabular}


Table II. Aerosol Size Measurement Methods

According to Their Operating Principle

\begin{tabular}{llll} 
Electric Mobility & Optical Property & Diffusivity & Inertial Property \\
\hline EAA (57) & OPC (58) & $\begin{array}{l}\text { Diffusion } \\
\text { batteries (59) }\end{array}$ & Impactors (62,63) \\
DMA (43-46) & $\begin{array}{l}\text { Photon } \\
\text { correlator }\end{array}$ & APS (64-66) \\
SEMS (47) & & Cyclones (42) \\
$\begin{array}{l}\text { Electromobility } \\
\text { spectrometer (48) }\end{array}$ & & \\
\hline
\end{tabular}


Table III. Summary of Aerosol Dynamics Simulation Routines

One-dimension Multidimension

\begin{tabular}{lll}
\hline & & \\
Single & AEROSOL (82) & \\
Component & AGRO (83) & \\
& CONFEMM (103) & \\
& COAGUL (84) & \\
& DISC (94) & \\
& CHARM (112) & \\
& MAEROS (94) & MULFEMM (101) \\
Multicomponent & ESMAP (95) & \\
& MGA (100) & \\
\hline
\end{tabular}


Table IV. Processes and Mechanisms Treated in AMPS

\begin{tabular}{lll} 
Process & Mechanism & $\begin{array}{l}\text { Numerical } \\
\text { Scheme }\end{array}$ \\
\hline $\begin{array}{l}\text { Condensation/ } \\
\text { Evaporation }\end{array}$ & $\begin{array}{l}\text { Diffusion-controiled } \\
\text { (Maxwell relation) } \\
\text { Volume reaction-controlled } \\
\text { gas-to-particle conversion }\end{array}$ & $\begin{array}{l}\text { Repeated upwind } \\
\text { difference, } \\
\text { Taylor-Galerkin }\end{array}$ \\
Coagulation & Brownian coagulation & $\begin{array}{l}\text { Product type } \\
\text { Gaussian quadrature }\end{array}$ \\
$\begin{array}{l}\text { Deposition } \\
\text { Source }\end{array}$ & $\begin{array}{l}\text { Linear deposition } \\
\text { User given, }\end{array}$ & $\begin{array}{l}\text { With Coagulation } \\
\text { Pre-specified }\end{array}$ \\
Nucleation & User given & With coagulation \\
Simultaneous & & Operator splitting \\
Processes & & \\
\hline
\end{tabular}


Table V. Comparison of Numerical Multicomponent Aerosol Dynamics Simulation Routines

\begin{tabular}{|c|c|c|c|c|c|}
\hline & MAEROS & ESMAP & MGA & MULFEMM & AMPS \\
\hline $\begin{array}{l}\text { Positive } \\
\text { Definite }\end{array}$ & Yes & Yes & Yes & No & Yes \\
\hline Conservation & Yes & Yes & Yes & No & Yes \\
\hline $\begin{array}{l}\text { Arbitrarily } \\
\text { Mixed } \\
\text { Particles }\end{array}$ & No & No & Yes & Yes & Yes \\
\hline $\begin{array}{l}\text { Numerical } \\
\text { Methods }\end{array}$ & $\begin{array}{l}\text { Sectional } \\
\text { method }\end{array}$ & $\begin{array}{l}\text { Sectional } \\
\text { method }\end{array}$ & $\begin{array}{l}\text { Moving } \\
\text { sectional } \\
\text { method }\end{array}$ & $\begin{array}{l}\text { Moving } \\
\text { finite } \\
\text { element } \\
\text { method }\end{array}$ & $\begin{array}{l}\text { RUD. } \\
\text { TGFE.M } \\
\text { Gaussian } \\
\text { quadrature }\end{array}$ \\
\hline Processes & $\begin{array}{l}\text { Coagulation } \\
\text { Cond/evap. } \\
\text { Source } \\
\text { Deposition }\end{array}$ & $\begin{array}{l}\text { Coagulation } \\
\text { Cond/evap. } \\
\text { Nucleation } \\
\text { Deposition } \\
\text { Source }\end{array}$ & Cond/evap. & Condensation & $\begin{array}{l}\text { Coagulation } \\
\text { Cond/evap. } \\
\text { Nucleation } \\
\text { Deposition } \\
\text { Source }\end{array}$ \\
\hline Reference & 82,83 & 95 & 102 & 103 & 79 \\
\hline
\end{tabular}

${ }^{1}$ RUD: the repeated upwind difference method TGFEM; the Taylor-Galerkin method. 


\section{FIGURE CAPTION}

Figure 1. An illustration of the effect of particle mixedness on aerosol dynamics: Hygroscopic difference affects particle growth by humidification.

Figure 2. An illustration of the effect of particle mixedness on aerosol dynamics: Light absorbing capacity difference affect light intensity.

Figure 3. Possible distributions of particles with the same mean mass fraction: Between two extremes, the external and the internal mixture, infinite number of distributions are possible.

Figure 4. Schematic diagram of the TDMA system. 

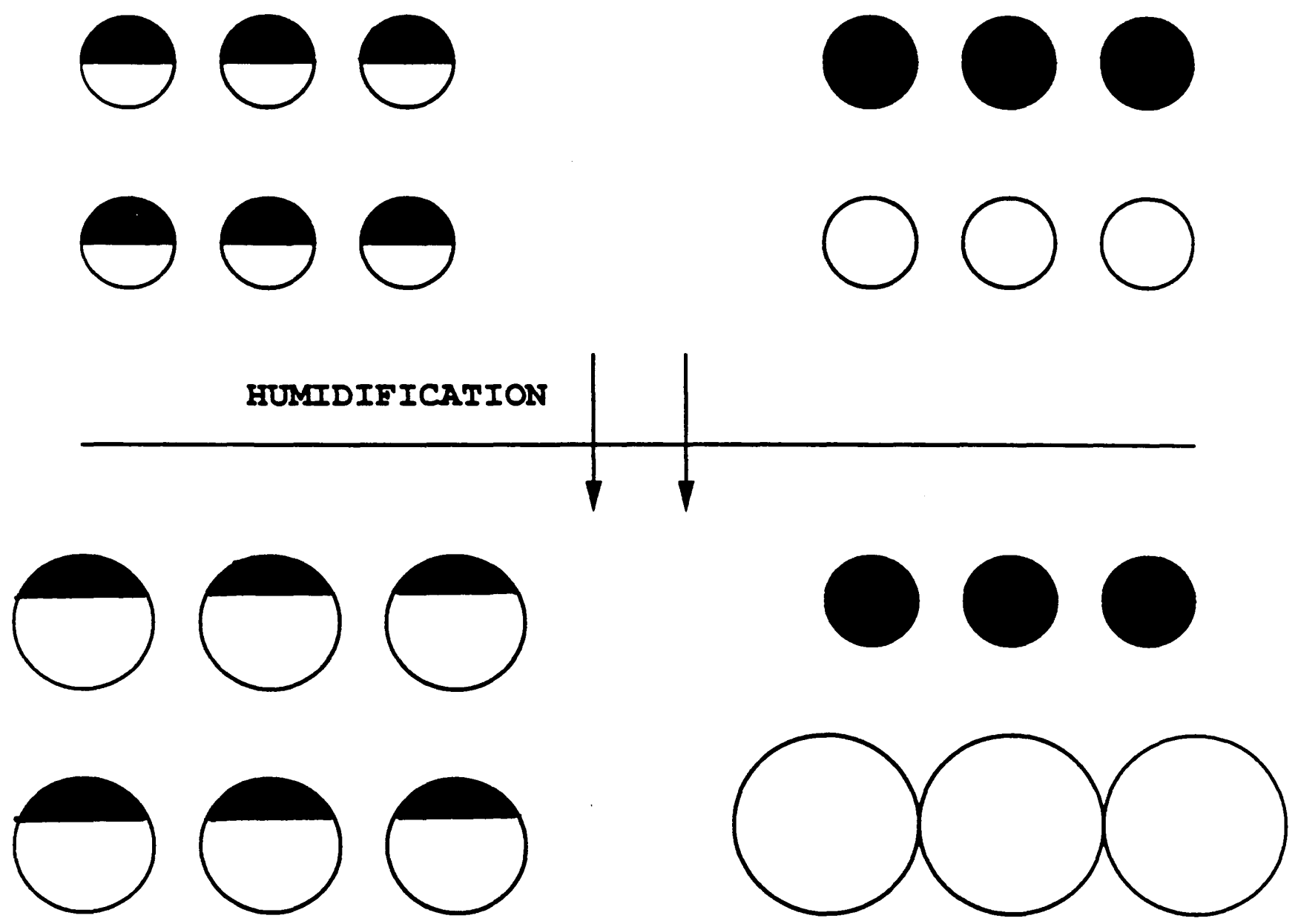

HYGROSCORIC COMPOUND

NON-HYGROSCORIC COMPOUND

Figure 1 

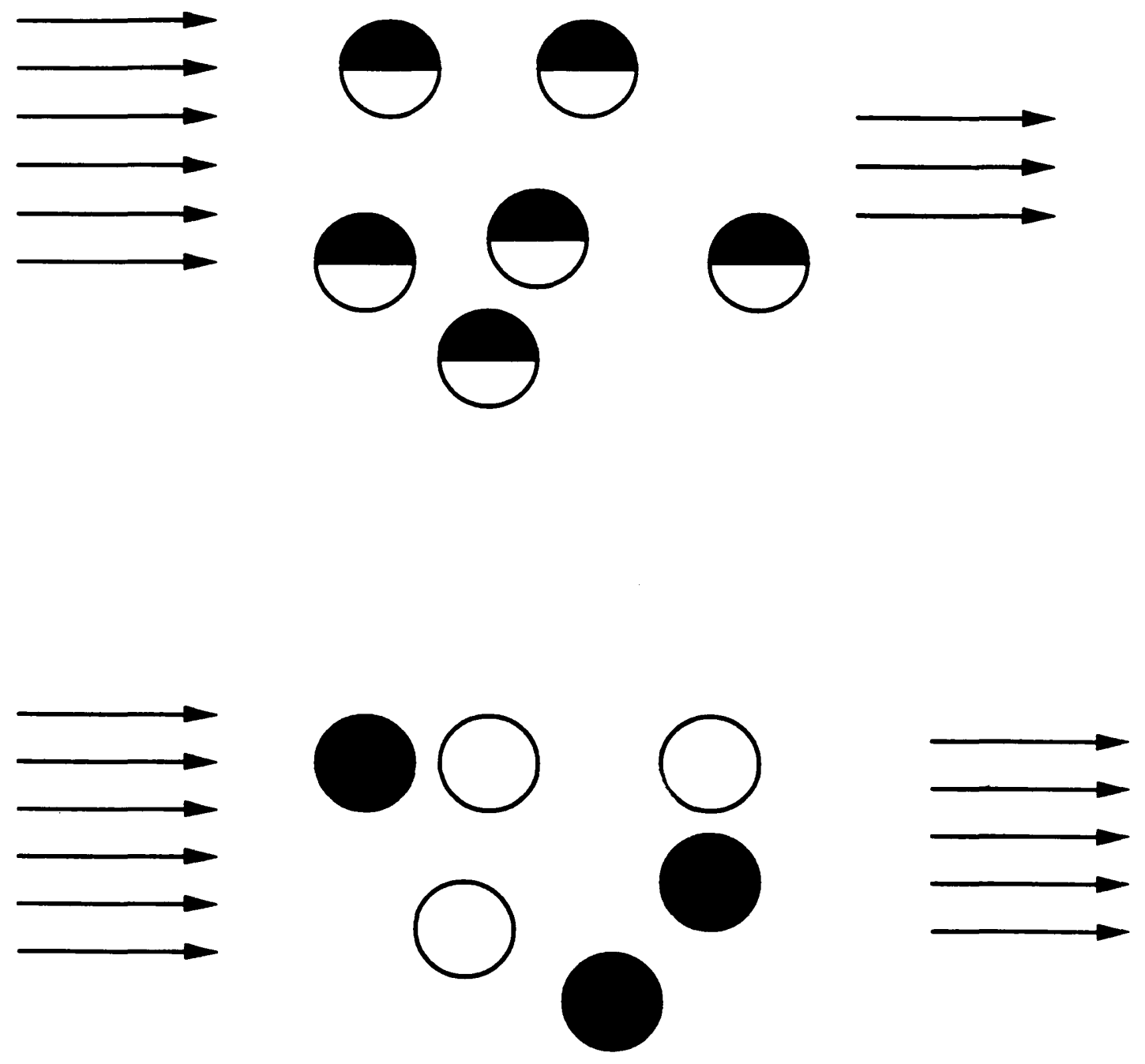

$\bigcirc$ IIGHT ABSORBING COMPOUND

IIGHT NON-ABSORBING COMPOUND

Figure 2 


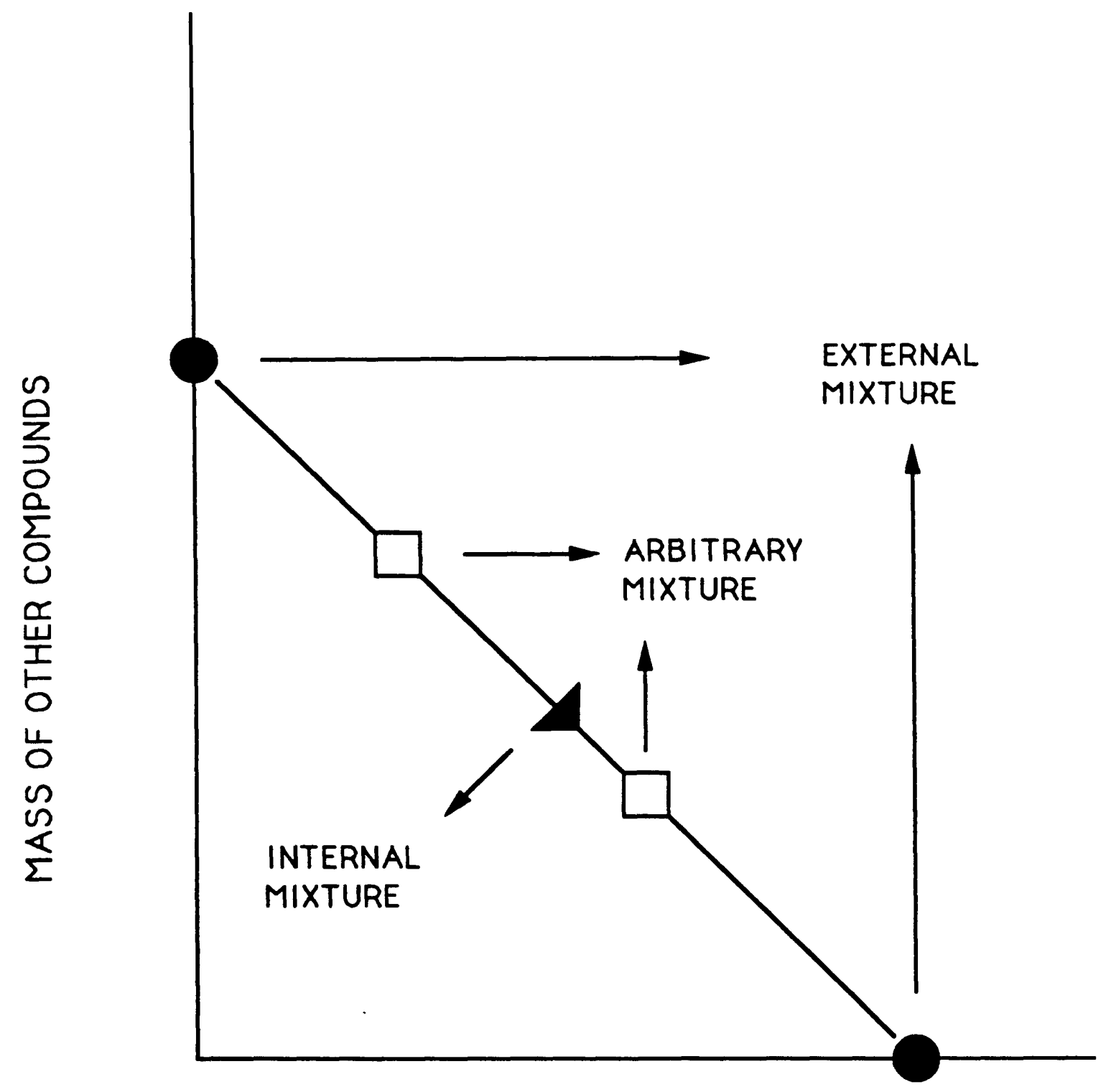

MASS OF THE COMPOUND OF INTEREST

Figure 3 


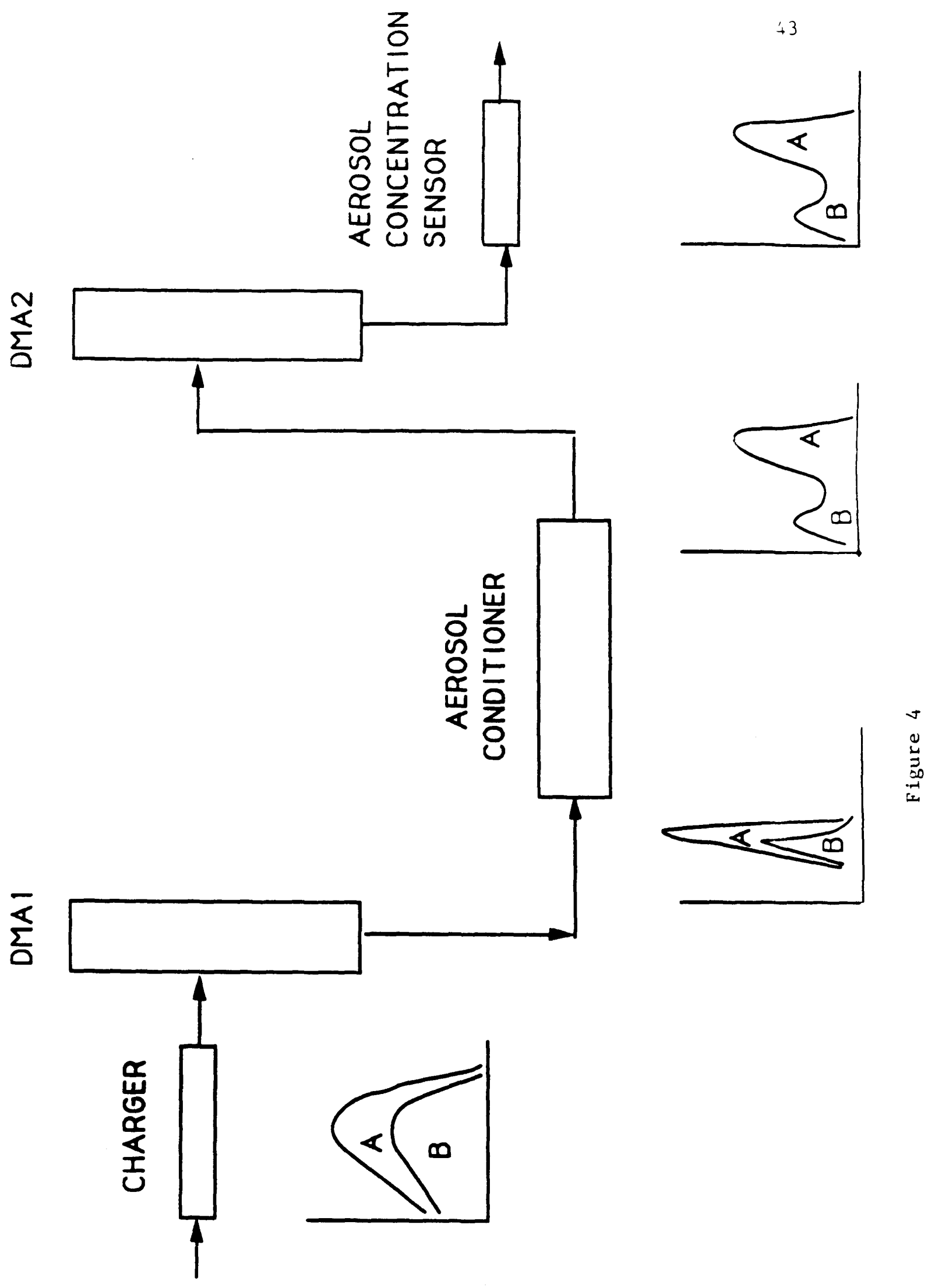


Chapter II

\section{SIMULATION OF MULTICOMPONENT AEROSOL CONDENSATION BY THE MOVING SECTIONAL METHOD}




\section{INTRODUCTION}

The aerosol general dynamic equation (GDE) presents unique computational challenges because of the inherently different nature of coagulation and condensation/ evaporation processes. In addition, the aerosol may be of a single component or may contain several components. The simplest problems to address computationally are those involving a single component and either, but not both, coagulation or condensation/ evaporation; the most demanding involve several components and simultaneous coagulation and condensation/ evaporation.

Considerable effort has been devoted to the development of numerical techniques for solving the aerosol GDE $(3,13)$. These efforts can be classified according to whether they address single component or multicomponent aerosols and which processes, notably coagulation and/or condensation/ evaporation, are occurring. Table I summarizes many of the previous approaches organized by these two criteria.

Most of the numerical methods that have been proposed are based on dividing the size distribution into elements or sections and assuming the form of the size distribution within these elements. The assumption of a constant distribution leads to the so-called sectional method $(1,5,6)$. Additionally, the elements or sections may remain fixed throughout the calculation or move. Fixed elements are desirable for representing coagulation; moving elements are preferable for condensation/ evaporation. The original sectional method, fixed elements with a uniform distribution within elements, is ideally suited for simulating coagulation but suffers from numerical diffusion errors when condensation/ evaporation occur.

The aerosol size distribution function during condensation/ evaporation is described by a first-order hyperbolic partial differential equation for which the occurrence of numerical dispersion and diffusion errors in numerical solutions is well known $(7,12)$. Discrete approximation of spatial derivatives of a hyperbolic differential equation may lead to significant numerical dispersion and diffusion errors. Numerical dispersion causes the modes of the numerical solution to propagate at speeds different from those of the exact solution, resulting in spurious oscillations around the true 
solution peak and/or negative values. Furth -rmore, numerical dispersion errors often cause instabilities since this error generally increases with time. Numerical diffusion errors cause the amplitude of the numerical solution to propagate in such a way that peaks in the solution dissipate. Note that this diffusion arises from numerical errors, and is not a physical phenomenon. Also note that when the amplitude of the numerical solution is larger than that of the exact solution, the numerical solution may become unstable.

Since aerosol condensation/ evaporation is particle number conserving, a numerical scheme addressed to condensation/ evaporation should not lose or gain particles from the computational domain during the simulation. (For the evaporation process, number conservation may be applied to particles larger than the critical size.)

Numerous schemes have been developed to solve hyperbolic partial differential equations numerically. Highly accurate schemes such as the Galerkin finite element method and the Lax-Wendroff method have little numerical diffusion errors but exhibit numerical dispersion errors for steep concentration profiles. Conversely, firstorder upwind differencing schemes are free of numerical dispersion errors but have numerical diffusion errors. Some numerical schemes such as the Flux-Corrected Transport (FCT) (24) and the Smolarkiewicz's repeated upwinding scheme (15) have been developed to eliminate numerical dispersion and reduce numerical diffusion. These schemes eliminate numerical dispersion by introducing diffusion terms and then subtracting the introduced diffusion contribution from the calculated quantities. Because all the schemes mentioned above calculate the transport of conserved quantities at fixed grids, i.e., using the Eulerian approach, numerical errors arising from the discrete approximation of spatial derivatives can not be eliminated, only reduced.

In Lagrangian methods, grids and computational cells move with the conserved quantities along the characteristics thus eliminating numerical errors due to the discrete approximation of spatial derivatives. The most significant difficulty of applying a Lagrangian approach to aerosol condensation/ evaporation is the distortion of the grids and cells that occurs as the different particle sizes grow or shrink at different 
rates. Some numerical schemes use the idea of remapping the functional values from the distorted Lagrangian grid onto a regular grid by interpolation at each time ste During interpolation, however, new numerical errors are introduced. Tsang and Brock (17) used a finite element method incorporating characteristics (21) for representing aerosol condensation and an Eulerian-Lagrangian scheme (10) for evaporation for single component aerosols. Seigneur et al. (13) also used a finite element method with characteristics. However, even though its global accuracy is good this method does not conserve particle number concentration as a function of time.

Gelbard (6) combined the sectional approach with the Lagrangian concept of a movable grid and developed a moving sectional method for simulating aerosol condensation/ evaporation of a single condensible species. In that method the boundaries of each particle size section move with time in accordance with the growth or shrinkage of particles at those boundaries. In that way numerical diffusion errors resulting from the need to apportion the particle size distribution over a set of fixed sections are avoided. Also, numerical dispersion errors resulting from the convection term are avoided since the convection term is automatically handled by solving the characteristic equations.

Single component aerosol dynamics involving either coagulation or condensation/ evaporation can be simulated quite accurately with methods in the literature. Single component aerosol dynamics involving both coagulation and condensation/ evaporation is considerably more difficult but can, nonetheless, be treated with available methods. The vast majority of papers in the literature address single component problems, and many are limited to condensation/ evaporation alone, In this latter case, an approach involving moving elements or sections is the preferred one. For multicomponent aerosol dynamics the only technique that has been developed and applied is the sectional method, with fixed sections. The representation of condensation/ evaporation is of course prone to numerical diffusion just as in the single component case.

There are several numerical schemes available to calculate multidimensional con- 
vection problems which have the same form as those of multicomponent aerosol condensation/ evaporation $(7,12)$, but the schemes require significant computer memory and computing time. For an $s$ component system, a finite difference method with $n$ grids for each component needs, for example, an $n^{s}$ grid point and a finite element method with $n$ cells for each component requires an $n^{s} \times n^{s}$ matrix system although the matrix is usually banded and/or sparse. Also one has to calculate $s$ condensation rate equations at each grid point $\left(s \times n^{s}\right.$ calculations for a finite difference method). Furthermore, to calculate the spatial and temporal derivatives with reasonable accuracy, rather time consuming schemes should be used, also requiring large computing time and memory. To calculate multicomponent aerosol dynamics due to condensation/ evaporation processes by conventional multidimensional convection schemes does not appear to be attractive. For this reason we adopt the sectional concept together with that of a Lagrangian grid.

Our goal in this work is to extend the concept of the moving sectional method to multicomponent aerosol condensation/ evaporation processes. To demonstrate the performance of the method we obtain analytical solutions to model problems of multicomponent condensation/ evaporation to which the numerical solutions may be compared. Accuracy and computational efficiency are further compared between the moving sectional method and the original, fixed grid sectional method.

Finally, limitations of the sectional method due to its inherent assumptions are discussed.

\section{GENERAL DYNAMIC EQUATION}

\section{FOR CONDENSATION/EVAPORATION}

The General Dynamic Equation (GDE) for multicomponent condensation/ evaporation processes is $(3)$

$$
\frac{\partial n(m, M, t)}{\partial t}+\sum_{i=1}^{s-1} \frac{\partial}{\partial m_{i}}\left[I_{i}(m, M, t) n(m, M, t)\right]
$$




$$
+\frac{\partial}{\partial M}[I(m, M, t) n(m, M, t)]=0
$$

where $M$ denotes the total mass of a particle and $m_{i}$ is the mass of the $i$-th component in the particle, $m=\left(m_{1}, \ldots, m_{s-1}\right)$, where $s$ is the number of components. $n(m, M, t) d m d M$ is the number of particles having total mass in the range $[M, M+d M]$, mass of component $i$ in the range $\left[m_{i}, m_{i}+d m_{i}\right], i=1, \ldots, s-1$, at the time $t . I_{i}$ is the time rate change of mass concentration of the $i$-th component and $I$ is the time rate change of the total mass concentration. The initial condition is the size-composition distribution at time zero,

$$
n(m, M, 0)=n_{0}(m, M)
$$

The boundary condition is given by

$$
n\left(m, M^{*}, t\right)=0
$$

where $M^{*}$ is the critical total mass below which no aerosol particles exist.

\section{SECTIONAL METHOD}

\subsection{Sectional Method Development}

The essential idea of the sectional method is to divide the aerosol size spectrum into $N$ sections and assume:

1. In a section, the total particle mass size distribution is uniform.

2. All particles in a section have identical composition.

The total mass concentration of the $l$-th section is defined as

$$
Q_{l}(t)=\int_{M_{l, l}}^{M_{l, u}} \int_{0}^{M} \ldots \int_{0}^{M} M n(m, M, t) d m d M
$$

where $M_{l, l}$ is the lower bound and $M_{l, u}$ is the upper bound of total mass in the $l$-th section.

By applying the first assumption, we get

$$
\int_{0}^{M} \cdots \int_{0}^{M} M n(m, M, t) d m=q_{l}(t) f^{\prime}(M)
$$


where $q_{l}(t)$ is a constant in the section and $f^{\prime}(M)=\frac{d f}{d M}$, where $f(M)$ is the size variable of interest. An example of $f(M)$ is $\ln \left(M / M_{0}\right)$.

Combining [4] and [5] gives

$$
\begin{aligned}
Q_{l}(t) & =\int_{M_{l, l}}^{M_{l, u}} q_{l}(t) f^{\prime}(M) d M \\
& =q_{l}(t)\left[f\left(M_{l, u}\right)-f\left(M_{l, l}\right)\right]
\end{aligned}
$$

The total number concentration of particles in the $l$-th section is given by

$$
N_{l}(t)=\int_{M_{l, l}}^{M_{l, u}} \int_{0}^{M} \ldots \int_{0}^{M} n(m, M, t) d m d M
$$

If one chooses $\ln M$ as the size variable, i.e., $f(M)$, then $N_{l}(t)$ is given by

$$
N_{l}(t)=\frac{Q_{l}(t)\left[\frac{1}{M_{l, l}}-\frac{1}{M_{l, u}}\right]}{\ln M_{l, u}-\ln M_{l, l}}
$$

By applying the second assumption,

$$
\frac{m_{i}}{M}=\text { constant } \quad M_{l, l} \leq m_{i} \leq M_{l, u} \quad i=1, \ldots, s-1
$$

The mass concentration of the $k$-th component in the $l$-th section, $Q_{l, k}(t)$, is given by

$$
\begin{aligned}
Q_{l, k}(t) & =\int_{M_{l, l}}^{M_{l, u}} \int_{0}^{M} \ldots \int_{0}^{M} m_{k} n(m, M, t) d m d M \\
& =\int_{M_{l, l}}^{M_{l, u}} M \int_{0}^{M} \cdots \int_{0}^{M}\left(\frac{m_{k}}{M}\right) n(m, M, t) d m d M \\
& =\left(\frac{m_{k}}{M}\right) Q_{l}(t) \quad k=1, \ldots, s-1
\end{aligned}
$$

\subsection{Moving Sectional Method}

During the condensation/ evaporation processes for a fixed volume, if the section boundaries move without loss or gain of particles in that section, the particle number concentration in a section must be constant with time. Thus $N_{l}(t)$ of Eq. [8] is constant, or $\frac{d N_{l}(t)}{d t}=0 \quad, l=1, \ldots, N$. Thus if the temporal variation of the section boundaries and each component mass in a section are known, one can calculate the 
evolution of total mass concentration of aerosol and mass concentration of each component in a section. If section boundaries follow the characteristics of condensation/ evaporation rates, particle number will be conserved.

In the moving sectional method all particles initially within a section remain there and the section boundaries move according to the particle growth equation, i.e., the characteristics. Therefore, all particles in a section can not escape the section boundaries. The section boundaries obey the characteristic equations shown below,

$$
\frac{d M_{j}}{d t}=I(m, M, t) \quad j=1, \ldots, 2 N
$$

where the index $j$ denotes the $2 N$ section boundaries.

The problem now is how to calculate component mass changes within each section as a result of the condensation/ evaporation processes. Each component in a section also varies according to the condensation rate equation,

$$
\frac{d m_{i}}{d t}=I_{i}(m, M, t) \quad i=1, \ldots, s-1
$$

Because of the second assumption of the sectional model, the ratio $\left(\frac{m_{1}}{M}\right)$ is constant in a section; therefore it is immaterial which value of $M$ is used to calculate $\left(\frac{m_{i}}{M}\right)$. Thus at each time step temporal component mass variations in a section are calculated by solving Eqs. [11] and [12] with a fixed value of $M$ in the section, and one can then calculate the multicomponent condensation process from Eqs. [8] and [10].

One important advantage of this method is that the moving sectional method can simulate particles of the same total mass but different compositions. Since composition: adjacent sections are discontinuous at the section boundary, condensation/ evaporation rates of each section at the boundary are different. As a result, sections can in principle overlap each other or holes in the calculation domain may develop between sections. In the examples to be considered here, we have assumed linear total mass condensation/ evaporation rates. In these cases no overlapping of sections or holes between sections develop, but with other growth rate equations, in the same total mass range, overlapping sections with different compositions could exist. 


\section{ANALYTIC SOLUTIONS FOR}

\section{MULTICOMPONENT AEROSOL CONDENSATION/EVAPORATION}

In this section, we will derive new analytical solutions for multicomponent aerosol condensation/ evaporation. These solutions will be used as a reference to evaluate the performance of the numerical scheme developed above.

Two problems of multicomponent aerosol condensation/ evaporation will be considered. Both problems are characterized by linear condensation/ evaporation rates. Although such rates describe the processes of volume reaction controlled gas-toparticle conversion (14), the main reason for their selection is to enable analytical solution of the GDE. The two problems differ in their initial aerosol size distributions; one problem has an exponential initial size distribution and the other has a log-normal initial size distribution.

Condensation/evaporation rate equations are taken to be linear in total mass and in each component,

$$
\begin{aligned}
& I=\alpha M \\
& I_{i}=\alpha_{i} m_{i} \quad i=1, \ldots, s-1
\end{aligned}
$$

Note that if the value of $\alpha_{i}$ is negative the $i$-th component evaporates. A three component system will be considered in the numerical examples to follow.

The derivation of the analytical solution for mass concentration together with definitions of the notation are given in the Appendix.

For the exponential initial size distribution case, the total mass concentration of the $l$-th section is

$$
\begin{aligned}
Q_{l}(t)= & \int_{M_{l, l}}^{M_{l, u}} \int_{0}^{M} \cdots \int_{0}^{M} \frac{N_{o}}{M_{o}} M \exp \left[-\frac{M}{M_{o}} e^{-\alpha t}-\alpha t\right] \\
& \times \prod_{i=1}^{s-1} \frac{1}{m_{i o}} \exp \left[-\frac{m_{i}}{m_{i o}} e^{-\alpha_{i} t}-\alpha_{i} t\right] d m d M \\
= & \int_{M_{l, l}}^{M_{l, u}} \frac{N_{o}}{M_{o}} M \exp \left[-\frac{M}{M_{o}} e^{-\alpha t}-\alpha t\right] \prod_{i=1}^{s-1}\left(1-\exp \left[-\frac{M}{m_{i o}} e^{-\alpha_{i} t}\right]\right) d M
\end{aligned}
$$


The mass concentration of $j$ th component in the $l$-th section is

$$
\begin{aligned}
Q_{l, j}(t)= & \int_{M_{l, l}}^{M_{l, u}} \frac{N_{o}}{M_{o}} \exp \left[-\frac{M}{M_{o}} e^{-\alpha t}-\alpha t\right]\left[\prod_{i=1, \neq j}^{s-1}\left(1-\exp \left[-\frac{M}{m_{i o}} e^{-\alpha_{i} t}\right]\right)\right] \\
& \times\left[\left(1-\exp \left[-\frac{M}{m_{j o}} e^{-\alpha_{j} t}\right]\right) m_{j o} e^{\alpha_{j} t}-M \exp \left[-\frac{M}{m_{j o}} e^{-\alpha_{j} t}\right]\right] d M
\end{aligned}
$$

For the log-normal initial distribution problem, the total mass concentration of the $l$-th section is

$$
\begin{aligned}
Q_{l}(t)= & \int_{M_{l, l}}^{M_{l, u}} \frac{N_{o}}{(2 \pi)^{1 / 2} \ln \sigma} \exp \left[-\frac{(\ln M-\ln \bar{M}-\alpha t)^{2}}{2 \ln ^{2} \sigma}\right] \\
& \times \prod_{i=1}^{s-1} \frac{1}{2}\left[1 \pm \operatorname{erf}\left(\frac{\left(\ln M-\ln \bar{m}_{i}-\alpha_{i} t\right)}{\sqrt{2} \ln \sigma_{i}}\right] d M\right.
\end{aligned}
$$

The mass concentration of the $j$ th component in the $l$-th section is

$$
\begin{aligned}
Q_{l, j}(t)= & \int_{M_{l, l}}^{M_{l, u}} \frac{N_{o}}{(2 \pi)^{1 / 2} M \ln \sigma} \exp \left[-\frac{(\ln M-\ln \bar{M}-\alpha t)^{2}}{2 \ln ^{2} \sigma}\right] \\
& \times\left[\prod _ { i = 1 , \neq j } ^ { s - 1 } \frac { 1 } { 2 } \left[1 \pm \operatorname{erf}\left(\frac{\left(\ln M-\ln \bar{n}_{i}-\alpha_{i} t\right)}{\sqrt{2} \ln \sigma_{i}}\right]\right.\right. \\
& \times \frac{1}{2} \exp \left[\ln \bar{m}_{j}+\alpha_{j} t+\frac{\ln ^{2} \sigma_{j}}{2}\right] \\
& \times\left[1 \pm \operatorname{erf}\left(\frac{\left(\ln M-\ln \bar{m}_{j}-\alpha_{j} t-\ln ^{2} \sigma_{j}\right)}{\sqrt{2} \ln \sigma_{i}}\right)\right] d M
\end{aligned}
$$

By using any appropriate numerical integration method, such as Gaussian quadrature, one can evaluate $Q_{l}(t)$ and $Q_{l, j}(t)$.

\section{NUMERICAL SOLUTIONS OF}

\section{MULTICOMPONENT AEROSOL CONDENSATION/EVAPORATION}

For generality and ease of calculation, non-dimensionalization will be used henceforth. Also, because of the wide range of magnitudes of the independent variables, a logarithmic transformation similar to that used by Gelbard and Seinfeld (2) is used:

$$
\begin{aligned}
& X=\ln \left(\frac{M}{M_{o}}\right) \\
& x_{i}=\ln \left(\frac{m_{i}}{M_{o}}\right) \quad i=1, \ldots, s-1
\end{aligned}
$$


The parameters chosen for the numerical simulations for the exponential initial distribution are $m_{1 o}=0.3, m_{2 o}=0.2$. For the log-normal initial distribution, the parameters employed are, for total number concentration, number median dimensionless mass of 3.0 and geometric standard deviation of 3.0. For components 1 and 2, number median dimensionless masses are 0.1 and 0.3 , and geometric standard deviations are 5.0 and 5.0 , respectively.

Two cases of condensation/ evaporation rates were solved for the model problems. Case 1 is a pure condensation process with condensation rate coefficient chosen as $\alpha=1.0 \mathrm{~s}^{-1}, \alpha_{1}=0.9 \mathrm{~s}^{-1}$ and $\alpha_{2}=1.1 \mathrm{~s}^{-1}$. The second case is one of mixed condensation/ evaporation. The evaporation rate coefficient of the first component is chosen as $\alpha_{1}=-0.1 \mathrm{~s}^{-1}$ and the condensation rates of total mass and component 2 are $\alpha=1.0 \mathrm{~s}^{-1}$ and $\alpha_{2}=1.7 \mathrm{~s}^{-1}$. The dimensionless time $\tau$ is defined as $\tau=\alpha t$ for these linear cases.

Ten sections were used for both the moving and fixed sectional method. The dimensionless calculation domain in terms of $\frac{M}{M_{0}}$ is $\left[10^{-2}, 10^{2}\right]$ for the exponential initial distribution and $\left[10^{-2}, 10^{5}\right]$ for the log-normal initial distribution.

The fixed sectional results were obtained according to the algorithm of Warren and Seinfeld (22). The average density of particles was $10^{6} \mathrm{~g} \mathrm{~m}^{-3}$ and the reference total mass was that of a particle of size $1 \mu \mathrm{m}$.

To assess the numerical errors due to the moving sectional method and to compare the results of the moving sectional method to those of the fixed sectional method, error sources from the time integration and the sectional method were carefully reduced or eliminated as much as possible. With linear condensation/ evaporation rates are assumed, the logarithmically transformed rate equations become zeroth-order ordinary differential equations. In such a case, the Euler method produces no numerical error due to time integration. Initial conditions calculated from the analytical expression were used for both analytical and numerical calculations to reduce numerical errors due to the assumptions of the sectional model.

Figure 1 shows a comparison between the analytical solution and the numerical 
solutions using sectional methods of total mass concentration for case 1 (all species condense) with exponential initial size distribution at dimensionless time 1. Excellent agreement between the moving sectional solution and the exact solution is obtained. The fixed sectional method with 10 sections gives numerical solutions that exhibit the effect of numerical diffusion errors. Increasing the number of sections by a factor of two increased somewhat the accuracy of numerical solution. Note the underpredictions at the peak and overpredictions at the larger particle end of the spectrum that are characteristics of numerical diffusion errors. Figures 2 and 3 show the results for case 1 and the exponential initial size distribution for dimensionless mass concentrations of components 1 and 2, respectively. Again, the moving sectional method shows excellent results, and the fixed sectional method exhibits numerical diffusion errors.

Figures 4,5 and 6 show the results for case 2 (component 1 evaporates, but others condense and the overall effect is condensation) with the exponential initial size distribution, As above, for the moving sectional method, good agreement with the exact solution is obtained though the numerical solutions underpredict at the smaller particle end of the spectrum. Doubling the number of sections did not appreciably increase the accuracy of the numerical solution using fixed sections.

To compare the results of the various approaches quantitatively, the median relative error,

$$
\text { Median Relative Error }=\operatorname{median}\left[\frac{\operatorname{abs}\left(\left.Q_{l}\right|_{\text {analytical }}-\left.Q_{l}\right|_{\text {numerical }}\right)}{\left.Q_{l}\right|_{\text {analytical }}} \times 100\right]
$$

the value of relative error below which one half of values of relative values lies, was computed. Since the dimensionless mass concentration varies widely (more than a factor of 10 in the computational domain), to use maximum absolute and therefore maximum relative errors among sections is not appropriate because a small shift of the numerical solution profile at the far right side of the domain results in a large error. Table II gives the median relative errors of the different methods for both cases.

In Table III, the ratio of computing times between the fixed and moving sectional methods is shown. The moving sectional method requires slightly more computing 
time than the fixed sectional method for the same number of sections, but less computing time than the fixed sectional method with twice the number of sections.

Since the moving sectional method incorporates a number conserving condition and uses condensation/ evaporation rates as the characteristics of the section boundaries movement, it retains the advantages of both the fixed sectional method and the finite element method incorporating characteristics, being free from numerical dispersion error while conserving number concentration. This method also has the advantage that it can be developed to treat multicomponent aerosols and both condensation and evaporation.

The sectional method (irrespective of whether the sections are moving or fixed) is not without limitations. The first assumption inherent in the method that total mass size distribution is uniform in a section is the simplest form of approximation of the size distribution and introduces numerical diffusion errors. Of course, such errors are inevitable in a fixed grid approach, even with higher order functional forms of approximation. Thus the first assumption in the sectional method can be viewed as a useful mathematical approximation.

The second assumption of the sectional method is that the compositions of each component are constant in a section, that is, particles of the same mass all have the same composition. This approximation introduces not only numerical errors but also may not be physically realistic. The moving sectional method can produce particles of the same size but different composition since sections can overlap each other and/or holes in the calculation domain may develop between sections. This property of the moving sectional method alleviates the limitations arising from the second assumption of the method.

As a final point, while moving elements offer many advantages for simulating condensation/ evaporation, they are not desirable for coagulation. It is therefore unlikely that a multicomponent coagulation and condensation/ evaporation method will involve moving sections. If one seeks to reduce potential numerical diffusion and at the same time to relax the assumption that all particles of the same size have the 
same composition, it may be necessary to attack the multicomponent GDE as a true multivariable equation.

\section{CONCLUSION}

The moving sectional method has been extended to multicomponent aerosol condensation/ evaporation processes with multiple condensible species using a Lagrangian approach and a mass conservation condition. This method accurately simulates model multicomponent condensation process for which analytical solutions are available. Limitations and applicability of the sectional method are discussed.

\section{ACKNOWLEDGMENT}

This work was supported by National Science Foundation grant ATM-8503103. 


\section{REFERENCES}

1. Bassett, M., Gelbard, F. and Seinfeld, J.H., Atmos. Environ. 15, 2395(1981).

2. Gelbard, F. and Seinfeld, J.H., J. Comp. Phys. 28, 357(1978).

3. Gelbard, F. and Seinfeld, J.H., J. Colloid Interface Sci. 68, 363(1979).

4. Gelbard, F., Tambour, Y. and Seinfeld, J.H., J. Colloid Interface Sci. 76, 541(1980).

5. Gelbard, F. and Seinfeld, J.H., J. Colloid Interface Sci. 78, 485(1980).

6. Gelbard, F., "Modeling Multicomponent Aerosol Particle Growth by Vapor Condensation," Aerosol Sci. Technol. (in press).

7. Lapidus, L. and Pinder, G.F., " Numerical Solution of Partial Differential Equations in Science and Engineering," John Wiley \& Sons, New York, 1982.

8. Middleton, P. and Brock, J., J. Colloid Interface Sci. 54, 249(1976).

9. Neiburger, M. and Chien, C.W., in "Physics of Precipitation" (Weickmann Ed.), p.194. American Geophysical Union, Washington, 1960.

10. Neuman, S.P., J. Comp. Phys. 41, 270(1981).

11. O'Neill, K., Water Resources Research 17, 1665(1981).

12. Oran, E.S. and Boris, J.P., " Numerical Simulation of Reactive Flow," Elsevier, New York, 1987.

13. Seigneur, C., Hudischewskyj, A.B., Seinfeld, J.H., Whitby, K.T., Whitby, E.R., Brock, J.R. and Barnes, H.M., Aerosol Sci. Technol. 5, 205(1986). 
14. Seinfeld, J.H., "Atmospheric Chemistry and Physics of Air Pollution," John Wiley \& Sons, New York, 1986.

15. Smolarkiewicz, P.K., J. Comp. Phys. 54, 325(1984).

16. Suck, S.H. and Brock, J.R., J. Aerosol Sci. 10, 581(1979).

17. Tsang, T.H. and Brock, J.R., Aerosol Sci. Technol. 2, 311(1983).

18. Tsang T.H. and Hippe, J.N., Aerosol Sci. Technol. 8, 265(1988).

19. Tsang T.H. and Korgaonkar, N., Aerosol Sci. Technol. 7, 317(1987).

20. Tsang, T.H. and Rao, J.R., Aerosol Sci. Technol. 9, 271(1988).

21. Varog̃lu, E. and Liam Finn, W.D., J. Comp. Phys. 34, 371(1980).

22. Warren, D.R. and Seinfeld, J. H., Aerosol Sci. Technol. 4, 31(1985).

23. Wu, J.J. and Flagan, R.C., J. Colloid Interface Sci. 123, 339(1988).

24. Zalesak, S.T., J. Comp. Phys. 31, 335(1979). 


\section{APPENDIX. ANALYTICAL SOLUTION FOR}

\section{MULTICOMPONENT AEROSOL CONDENSATION/EVAPORATION}

The general multicomponent condensation equation is given by Eq. [1]. The analytical solution of Eq. [1] can be expressed as

$$
n(m, M, t)=\exp \left[-\sum_{i=1}^{s-1} \alpha_{i} t-\alpha t\right] n_{o}\left(m_{1} e^{-\alpha_{1} t}, \ldots, m_{s-1} e^{-\alpha_{s-1} t}, M e^{-\alpha t}\right) \quad[A-1]
$$

The exponential initial distribution is given by

$$
n_{o}(m, M)=\frac{N_{o}}{M_{o}} \exp \left[-\frac{M}{M_{o}}\right] \prod_{i=1}^{s-1} \frac{1}{m_{i o}} \exp \left[-\frac{m_{i}}{m_{i o}}\right]
$$

and the analytical solution for the exponential initial distribution is

$$
n(m, M, t)=\frac{N_{o}}{M_{o}} \exp \left[-\frac{M}{M_{o}} e^{-\alpha t}-\alpha t\right] \prod_{i=1}^{s-1} \frac{1}{m_{i o}} \exp \left[-\frac{m_{i}}{m_{i o}} e^{-\alpha_{i} t}-\alpha_{i} t\right] \quad[A-3]
$$

Then the total mass concentration of the $l$-th section is

$$
\begin{aligned}
Q_{l}(t)= & \int_{M_{l, l}}^{M_{l, u}} \int_{0}^{M} \cdots \int_{0}^{M} \frac{N_{o}}{M_{o}} M \exp \left[-\frac{M}{M_{o}} e^{-\alpha t}-\alpha t\right] \\
& \times \prod_{i=1}^{s-1} \frac{1}{m_{i o}} \exp \left[-\frac{m_{i}}{m_{i o}} e^{-\alpha_{i} t}-\alpha_{i} t\right] d m d M \\
= & \int_{M_{l, l}}^{M_{l, u}} \frac{N_{o}}{M_{o}} M \exp \left[-\frac{M}{M_{o}} e^{-\alpha t}-\alpha t\right] \prod_{i=1}^{s-1}\left(1-\exp \left[-\frac{M}{m_{i o}} e^{-\alpha_{i} t}\right]\right) d M[A-4]
\end{aligned}
$$

The mass concentration of $j$-th component in the $l$-th section is

$$
\begin{aligned}
Q_{l, j}(t)= & \int_{M_{l, l}}^{M_{l, u}} \frac{N_{o}}{M_{o}} \exp \left[-\frac{M}{M_{o}} e^{-\alpha t}-\alpha t\right] \\
& \times\left[\int_{0}^{M} \cdots \int_{0}^{M} \prod_{i=1}^{s-1} \frac{m_{j}}{m_{i o}} \exp \left[-\frac{m_{i}}{m_{i o}} e^{-\alpha_{i} t}-\alpha_{i} t\right] d m\right] d M
\end{aligned}
$$

If $i \neq j$, the integration is the same as Eq. [A-4]. If $i=j$, using integration by parts,

$$
\begin{aligned}
& \int_{0}^{M} \frac{m_{j}}{m_{j o}} \exp \left[-\frac{m_{j}}{m_{j o}} e^{-\alpha_{j} t}-\alpha_{j} t\right] d m_{j} \\
= & -\left[m_{j} \exp \left[-\frac{m_{j}}{m_{j o}} e^{-\alpha_{j} t}\right]_{o}^{M}+\int_{0}^{M} \exp \left[-\frac{m_{j}}{m_{j o}} e^{-\alpha_{j} t}\right] d m_{j}\right. \\
= & -M \exp \left[-\frac{M}{m_{j o}} e^{-\alpha_{j} t}\right]+m_{j o} e^{\alpha_{j} t}\left(1-\exp \left[-\frac{M}{m_{j o}} e^{-\alpha_{j} t}\right]\right)
\end{aligned}
$$


Therefore,

$$
\begin{aligned}
Q_{l, j}(t)= & \int_{M_{l, l}}^{M_{l, u}} \frac{N_{o}}{M_{o}} \exp \left[-\frac{M}{M_{o}} e^{-\alpha t}-\alpha t\right]\left[\prod_{i=1, \neq j}^{s-1}\left(1-\exp \left[-\frac{M}{m_{i o}} e^{-\alpha_{i} t}\right]\right)\right] \\
& \times\left[\left(1-\exp \left[-\frac{M}{m_{j o}} e^{-\alpha_{j} t}\right]\right) m_{j o} e^{\alpha_{j} t}-M \exp \left[-\frac{M}{m_{j o}} e^{-\alpha, t}\right]\right] d M[A-7]
\end{aligned}
$$

The log-normal initial distribution is given by

$$
\begin{aligned}
n_{o}(m, M)= & \frac{N_{o}}{(2 \pi)^{1 / 2} M \ln \sigma} \exp \left[-\frac{(\ln M-\ln \bar{M})^{2}}{2 \ln ^{2} \sigma}\right] \\
& \times \prod_{i=1}^{s-1} \frac{1}{(2 \pi)^{1 / 2} m_{i} \ln \sigma_{i}} \exp \left[-\frac{\left(\ln m_{i}-\ln \bar{m}_{i}\right)^{2}}{2 \ln ^{2} \sigma_{i}}\right]
\end{aligned}
$$

where $\bar{M}$ and $\bar{m}_{i}$ are number median masses and $\sigma$ and $\sigma_{i}$ are geometric standard deviations

For the log-normal initial distribution problem, the analytical solution is

$$
\begin{aligned}
n(m, M, t)= & \frac{N_{o}}{(2 \pi)^{1 / 2} M \ln \sigma} \exp \left[-\frac{(\ln M-\ln \bar{M}-\alpha t)^{2}}{2 \ln ^{2} \sigma}\right] \\
& \times \prod_{i=1}^{s-1} \frac{1}{(2 \pi)^{1 / 2} m_{i} \ln \sigma_{i}} \exp \left[-\frac{\left(\ln m_{i}-\ln \bar{m}_{i}-\alpha_{i} t\right)^{2}}{2 \ln ^{2} \sigma_{i}}\right] \quad[A-9]
\end{aligned}
$$

Then the total mass concentration of the $l$ th section is

$$
\begin{aligned}
Q_{l}(t)= & \int_{M_{l, l}}^{M_{l, u}} \frac{N_{o} M}{(2 \pi)^{1 / 2} M \ln \sigma} \exp \left[-\frac{(\ln M-\ln \bar{M}-\alpha t)^{2}}{2 \ln ^{2} \sigma}\right]\left[\int_{0}^{M} \ldots \int_{0}^{M}\right. \\
& \left.\prod_{i=1}^{s-1} \frac{1}{(2 \pi)^{1 / 2} m_{i} \ln \sigma_{i}} \exp \left[-\frac{\left(\ln m_{i}-\ln \bar{m}_{i}-\alpha_{i} t\right)^{2}}{2 \ln ^{2} \sigma_{i}}\right] d m\right] d M \quad[A-10]
\end{aligned}
$$

By changing variables, $u_{i}=\ln m_{i}$, and using the definition of the error function,

$$
\begin{aligned}
& \int_{0}^{M} \cdots \int_{0}^{M} \prod_{i=1}^{s-1} \frac{1}{(2 \pi)^{1 / 2} m_{i} \ln \sigma_{i}} \exp \left[-\frac{\left(\ln m_{i}-\ln \bar{m}_{i}-\alpha_{i} t\right)^{2}}{2 \ln ^{2} \sigma_{i}}\right] d m \\
= & \int_{-\infty}^{\ln M} \cdots \int_{-\infty}^{\ln M} \prod_{i=1}^{s-1} \frac{1}{(2 \pi)^{1 / 2} \ln \sigma_{i}} \exp \left[-\frac{\left(u_{i}-\ln \bar{m}_{i}-\alpha_{i} t\right)^{2}}{2 \ln ^{2} \sigma_{i}}\right] d u \\
= & \prod_{i=1}^{s-1} \frac{1}{2}\left[1 \pm \operatorname{erf}\left(\frac{\left(\ln M-\ln \bar{m}_{i}-\alpha_{i} t\right)}{\sqrt{2} \ln \sigma_{i}}\right]\right.
\end{aligned}
$$

where the plus sign is used if $\ln M \geq 0$, and the minus sign is used if $\ln M<0$. The error function is defined as

$$
\operatorname{erf}(z)=\frac{2}{\sqrt{\pi}} \int_{0}^{z} \exp \left[-t^{2}\right] d t
$$


So $Q_{l}(t)$ is

$$
\begin{aligned}
Q_{l}(t)= & \int_{M_{l, l}}^{M_{l, u}} \frac{N_{o}}{(2 \pi)^{1 / 2} \ln \sigma} \exp \left[-\frac{(\ln M-\ln \bar{M}-\alpha t)^{2}}{2 \ln ^{2} \sigma}\right] \\
& \times \prod_{i=1}^{s-1} \frac{1}{2}\left[1 \pm \operatorname{erf}\left(\frac{\left(\ln M-\ln \bar{m}_{i}-\alpha_{i} t\right)}{\sqrt{2} \ln \sigma_{i}}\right] d M\right.
\end{aligned}
$$

The mass concentration of the $j$-th component in the $l$-th section is

$$
\begin{aligned}
Q_{l, j}(t)= & \int_{M_{l, l}}^{M_{l, u}} \frac{N_{o}}{(2 \pi)^{1 / 2} M \ln \sigma} \exp \left[-\frac{(\ln M-\ln \bar{M}-\alpha t)^{2}}{2 \ln ^{2} \sigma}\right]\left[\int_{0}^{M} \cdots \int_{0}^{M}\right. \\
& \left.\prod_{i=1}^{s-1} \frac{m_{j}}{(2 \pi)^{1 / 2} m_{i} \ln \sigma_{i}} \exp \left[-\frac{\left(\ln m_{i}-\ln \bar{m}_{i}-\alpha_{i} t\right)^{2}}{2 \ln ^{2} \sigma_{i}}\right] d m\right] d M \quad[A-14]
\end{aligned}
$$

If $i \neq j$, the integration is the same as Eq. [A-11]. If $i=j$, expanding the exponential term and completing the square,

$$
\begin{aligned}
& \int_{0}^{M} \frac{m_{j}}{(2 \pi)^{1 / 2} m_{j} \ln \sigma_{j}} \exp \left[-\frac{\left(\ln m_{j}-\ln \bar{m}_{j}-\alpha_{j} t\right)^{2}}{2 \ln ^{2} \sigma_{j}}\right] d m_{j} \\
= & \int_{-\infty}^{\ln M} \frac{e^{u_{j}}}{(2 \pi)^{1 / 2} \ln \sigma_{j}} \exp \left[-\frac{\left(u_{j}-\ln \bar{m}-\alpha_{i} t\right)^{2}}{2 \ln ^{2} \sigma_{j}}\right] d u_{j} \\
= & \int_{-\infty}^{\ln M} \frac{1}{(2 \pi)^{1 / 2} \ln \sigma_{j}} \exp \left[\ln \bar{m}+\alpha_{i} t+\frac{\ln ^{2} \sigma_{j}}{2}\right] \exp \left[-\frac{\left(u_{j}-\left(\ln \bar{m}+\alpha_{i} t+\ln ^{2} \sigma_{j}\right)\right)^{2}}{2 \ln ^{2} \sigma_{j}}\right] d u_{j} \\
= & \frac{1}{2} \exp \left[\ln \bar{m}_{j}+\alpha_{j} t+\frac{\ln ^{2} \sigma_{j}}{2}\right]\left[1 \pm \operatorname{erf}\left(\frac{\left(\ln M-\ln \bar{m}_{j}-\alpha_{j} t-\ln ^{2} \sigma_{j}\right)}{\sqrt{2} \ln \sigma_{i}}\right)\right][A-15]
\end{aligned}
$$

where the plus sign is used if $\ln M \geq 0$, and the minus sign is used if $\ln M<0$.

Thus $Q_{l, j}(t)$ is,

$$
\begin{aligned}
Q_{l, j}(t)= & \int_{M_{l, l}}^{M_{l, u}} \frac{N_{o}}{(2 \pi)^{1 / 2} M \ln \sigma} \exp \left[-\frac{(\ln M-\ln \bar{M}-\alpha t)^{2}}{2 \ln ^{2} \sigma}\right] \\
& \times\left[\prod _ { i = 1 , \neq j } ^ { s - 1 } \frac { 1 } { 2 } \left[1 \pm \operatorname{erf}\left(\frac{\left(\ln M-\ln \bar{m}_{i}-\alpha_{i} t\right)}{\sqrt{2} \ln \sigma_{i}}\right]\right.\right. \\
& \times \frac{1}{2} \exp \left[\ln \bar{m}_{j}+\alpha_{j} t+\frac{\ln ^{2} \sigma_{j}}{2}\right] \\
& \times\left[1 \pm \operatorname{erf}\left(\frac{\left(\ln M-\ln \bar{m}_{j}-\alpha_{j} t-\ln ^{2} \sigma_{j}\right)}{\sqrt{2} \ln \sigma_{i}}\right)\right] d M
\end{aligned}
$$


Table I. Approaches to the Numerical Simulation of Aerosol Dynamics

Process Single component Multicomponent

Condensation/ Lagrangian calculation (9)

evaporation

only

Finite element method incorporating characteristics

and combined Eulerian-

Lagrangian scheme (17)

Galerkin finite element method

and Smolarkiewicz repeated

upwinding scheme (19)

Coagulation

only

Condensation/ evaporation

and

Coagulation

$\mathrm{J}$-space transformation with cubic spline method (16)

Sectional method (4)

J-space transformation with cubic spline method (8)

Orthogonal collocation and cubic spline on finite element method (2)

Combine (16) and (17) (18)

Sectional method $(22,23)$
Moving sectional method

(6 and this work)

Sectional method (5)

Sectional method (1) 
Table II. Comparison of Median Relative Error, Eq. [21 $]^{1}$ of Total Dimensionless Mass Concentration, at $\tau=1$

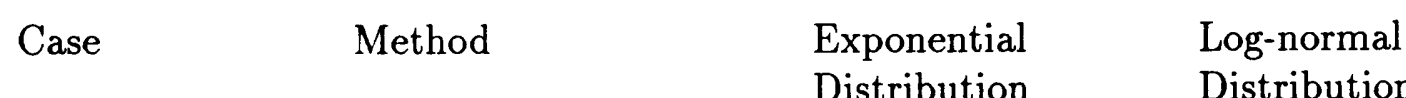

Moving sectional $\quad 0.214 \times 10^{\circ} \quad 0.166 \times 10^{\circ}$
method

$\begin{array}{llll}\text { Case } 1^{2} & \text { Fixed sectional } & 0.640 \times 10^{3} & 0.354 \times 10^{4}\end{array}$ method

$\begin{array}{lll}\text { Fixed sectional } & 0.305 \times 10^{3} & 0.203 \times 10^{4}\end{array}$ method (20 sections)

Moving sectional $\quad 0.560 \times 10^{0} \quad 0.195 \times 10^{1}$ method
Case $2^{3}$
Fixed sectional
$0.445 \times 10^{3}$
$0.425 \times 10^{4}$ method

Fixed sectional

$0.200 \times 10^{3}$

$0.215 \times 10^{4}$

method

(20 sections)

\footnotetext{
${ }^{1}$ Median Relative Error $=\operatorname{median}\left[\frac{\mathrm{abs}\left(\left.Q_{l}\right|_{\text {analytical }}-\left.Q_{l}\right|_{\text {numerical }}\right)}{\left.Q_{l}\right|_{\text {analytical }}} \times 100\right]$

2 all components condense

${ }^{3}$ component one evaporates, but others condense
} 
Table III. Comparison of Ratio of Computing Times at $\tau=1$

\begin{tabular}{llll} 
Case & Method & $\begin{array}{l}\text { Exponential } \\
\text { Distribution }\end{array}$ & $\begin{array}{l}\text { Log-normal } \\
\text { Distribution }\end{array}$ \\
\hline & $\begin{array}{l}\text { Moving sectional } \\
\text { method }\end{array}$ & 1 & 1 \\
Case $1^{1}$ & $\begin{array}{l}\text { Fixed sectional } \\
\text { method }\end{array}$ & 0.852 & 0.897 \\
& $\begin{array}{l}\text { Fixed sectional } \\
\text { method } \\
\text { (20 sections) }\end{array}$ & 1.356 & 1.402 \\
\hline Case $2^{2}$ & $\begin{array}{l}\text { Moving sectional } \\
\text { method }\end{array}$ & 1 & 1 \\
& $\begin{array}{l}\text { Fixed sectional } \\
\text { method }\end{array}$ & 0.916 & 1.769 \\
& $\begin{array}{l}\text { Fixed sectional } \\
\text { method } \\
\text { (20 sections) }\end{array}$ & 1.583 & 0.923 \\
\hline
\end{tabular}

\footnotetext{
${ }^{1}$ all components condense

${ }^{2}$ one component evaporates, but others condense
} 


\section{FIGURE CAPTIONS}

Figure 1. Comparison of analytical solution (solid line, left one is the initial size distribution) and sectional solutions of dimensionless total mass concentration for case 1 with exponential initial size distribution at dimensionless time 1. ( MS(10): moving sectional method with 10 sections, FS(10): fixed sectional method with 10 sections, $\mathrm{FS}(20)$ : fixed sectional method with 20 sections).

Figure 2. Comparison of analytical solution (solid line, left one is the initial size distribution) and sectional solutions of dimensionless mass concentration of component 1 for case 1 with exponential initial size distribution at dimensionless time 1 . ( MS(10): moving sectional method with 10 sections, FS(10): fixed sectional method with 10 sections, $\mathrm{FS}(20)$ : fixed sectional method with 20 sections).

Figure 3. Comparison of analytical solution (solid line, left one is the initial size distribution) and sectional solutions of dimensionless mass concentration of component 2 for case 1 with exponential initial size distribution at dimensionless time 1 . ( MS(10): moving sectional method with 10 sections, FS(10): fixed sectional method with 10 sections, $\mathrm{FS}(20)$ : fixed sectional method with 20 sections).

Figure 4. Comparison of analytical solution (solid line) and sectional solutions of dimensionless total mass concentration for case 2 with exponential initial size distribution at dimensionless time 1. (MS(10): moving sectional method with 10 sections, FS(10): fixed sectional method with 10 sections, FS(20): fixed sectional method with 20 sections).

Figure 5. Comparison of analytical solution (solid line) and sectional solutions of dimensionless mass concentration of component 1 for case 2 with exponential initial size distribution at dimensionless time 1. ( MS(10): moving sectional method with 10 sections, FS(10): fixed sectional method with 10 sections, FS(20): fixed sectional method with 20 sections). 
Figure 6. Comparison of analytical solution (solid line) and sectional solutions of dimensionless mass concentration of component 2 for case 2 with exponential initial size distribution at dimensionless time 1. ( MS(10): moving sectional method with 10 sections, $\mathrm{FS}(10)$ : fixed sectional method with 10 sections, $\mathrm{FS}(20)$ : fixed sectional method with 20 sections). 
68

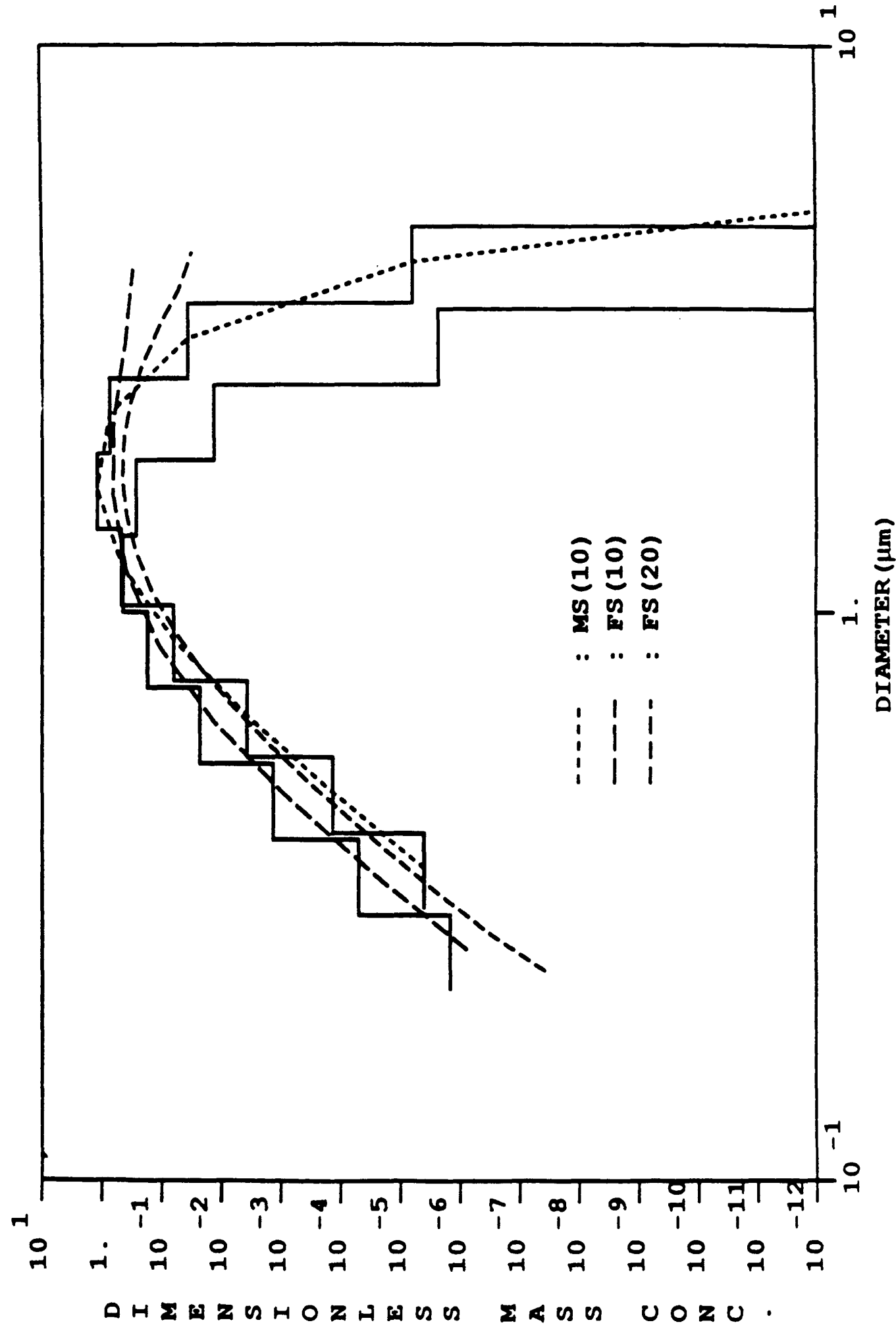


69

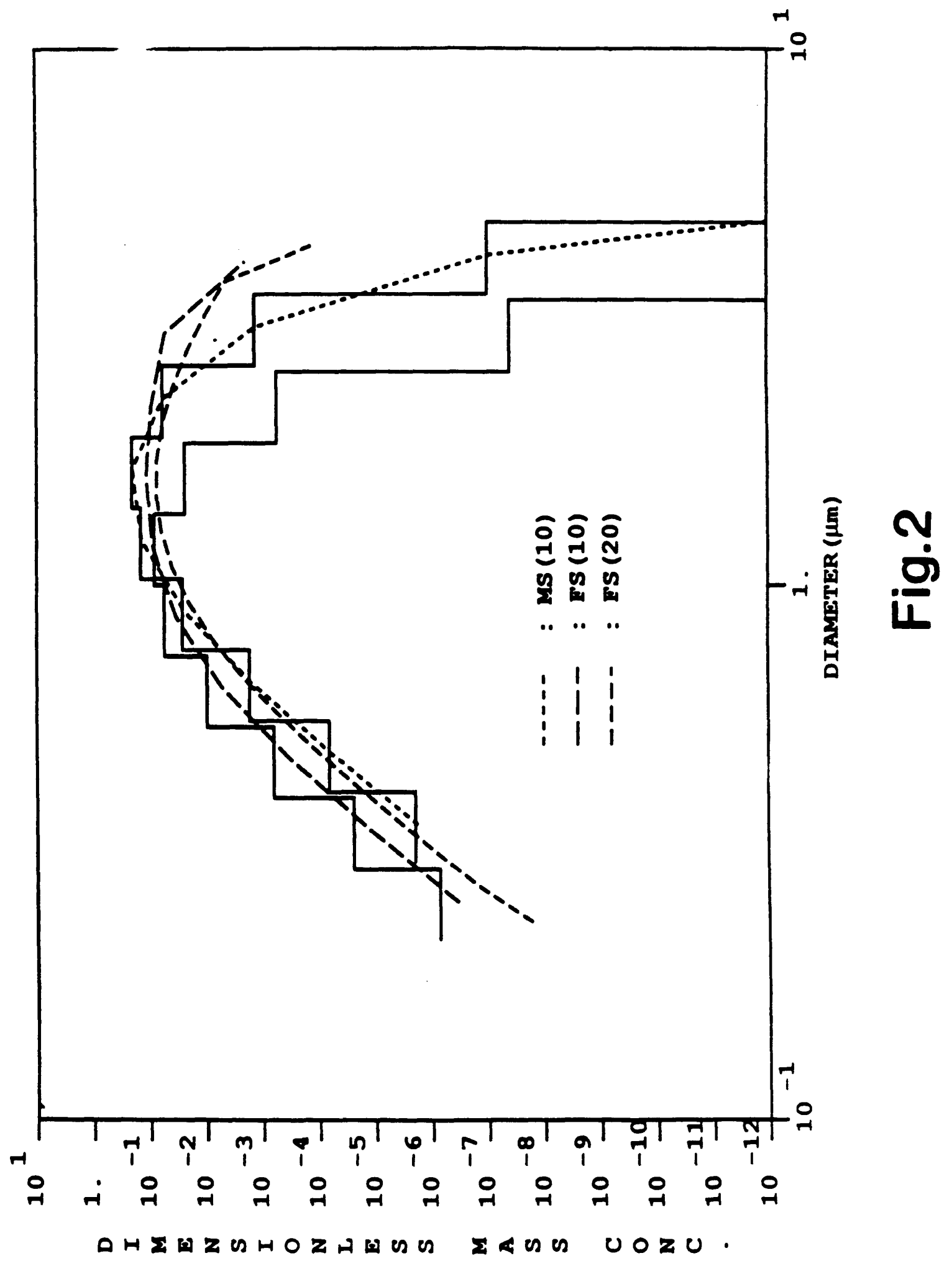




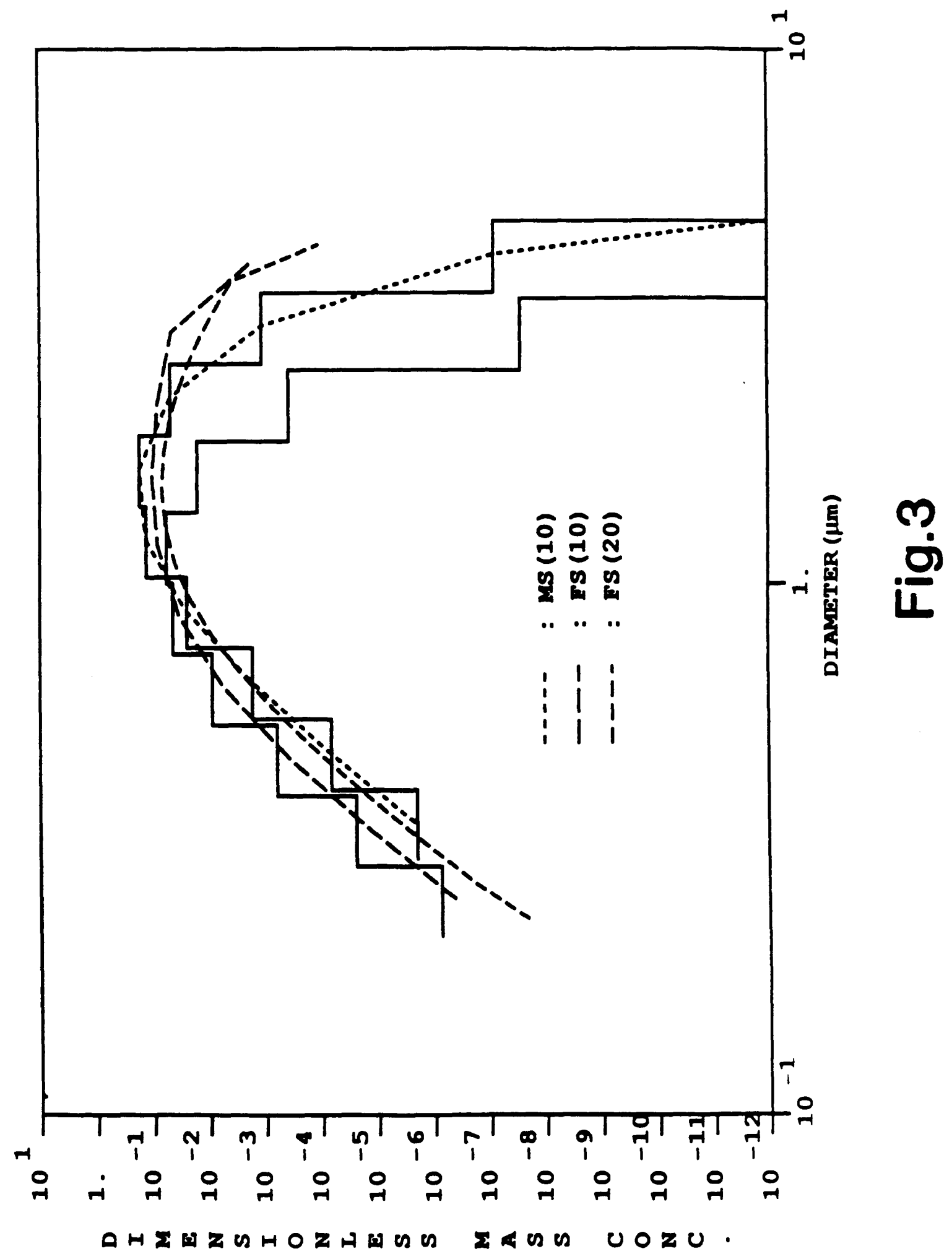


71

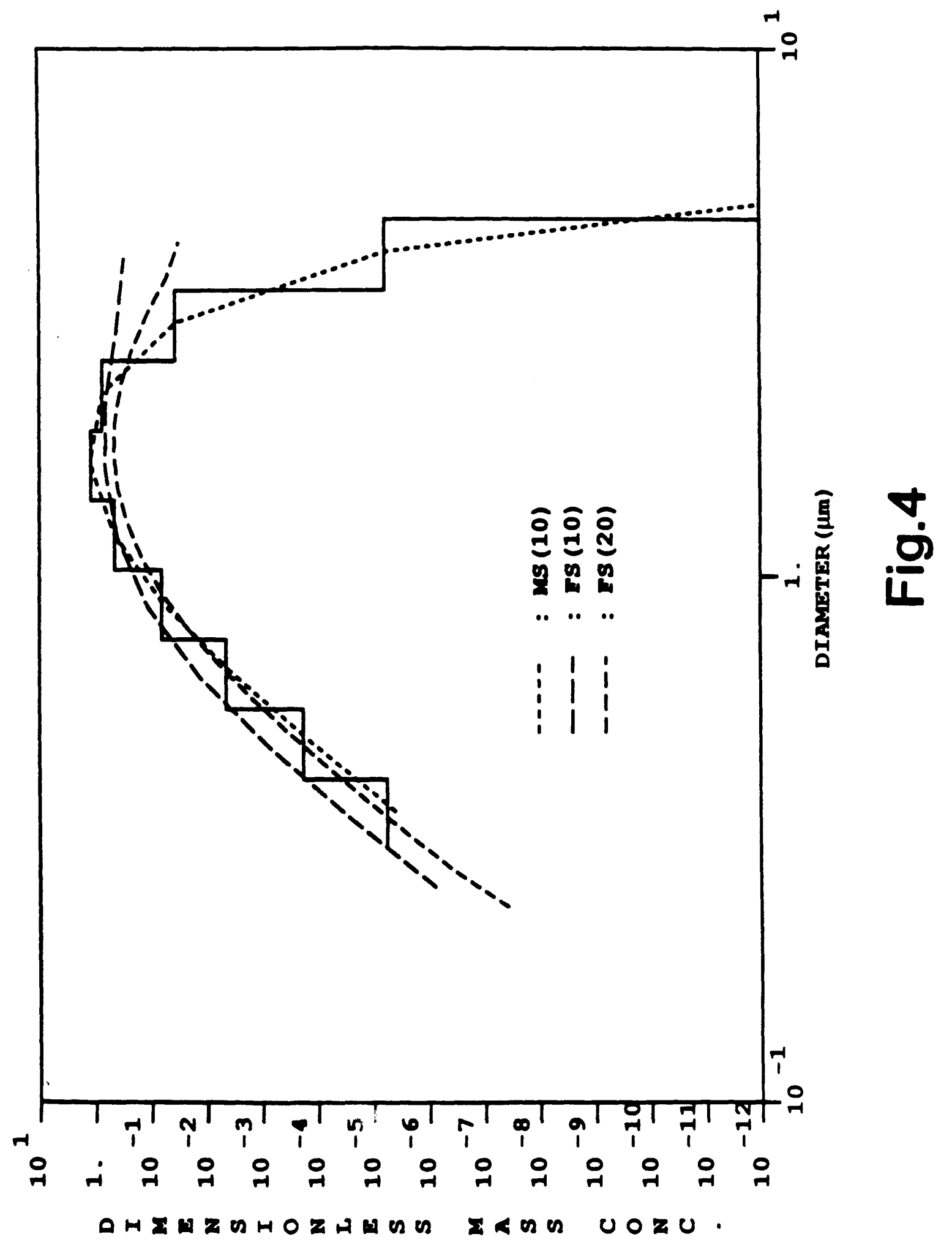


72

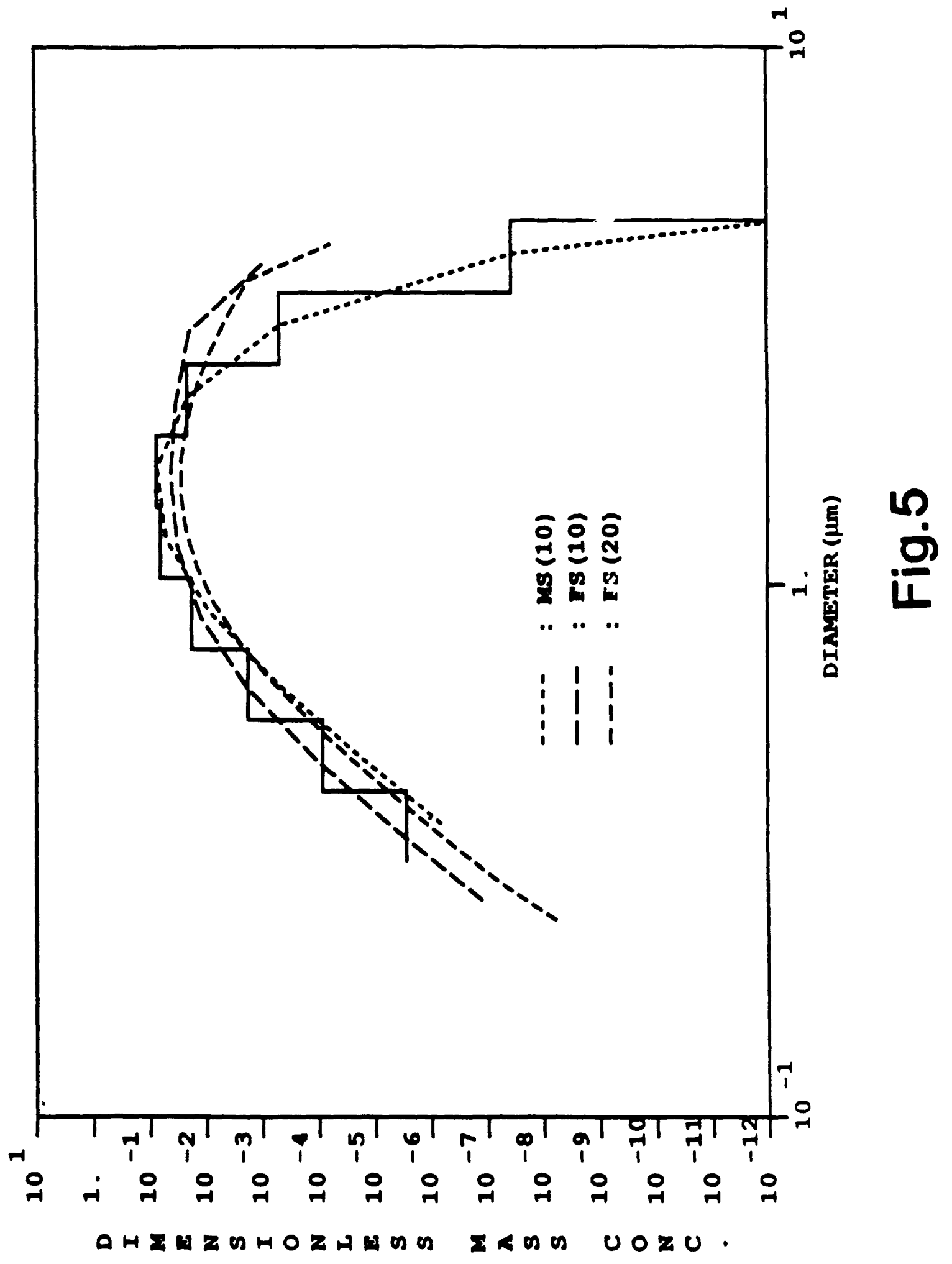




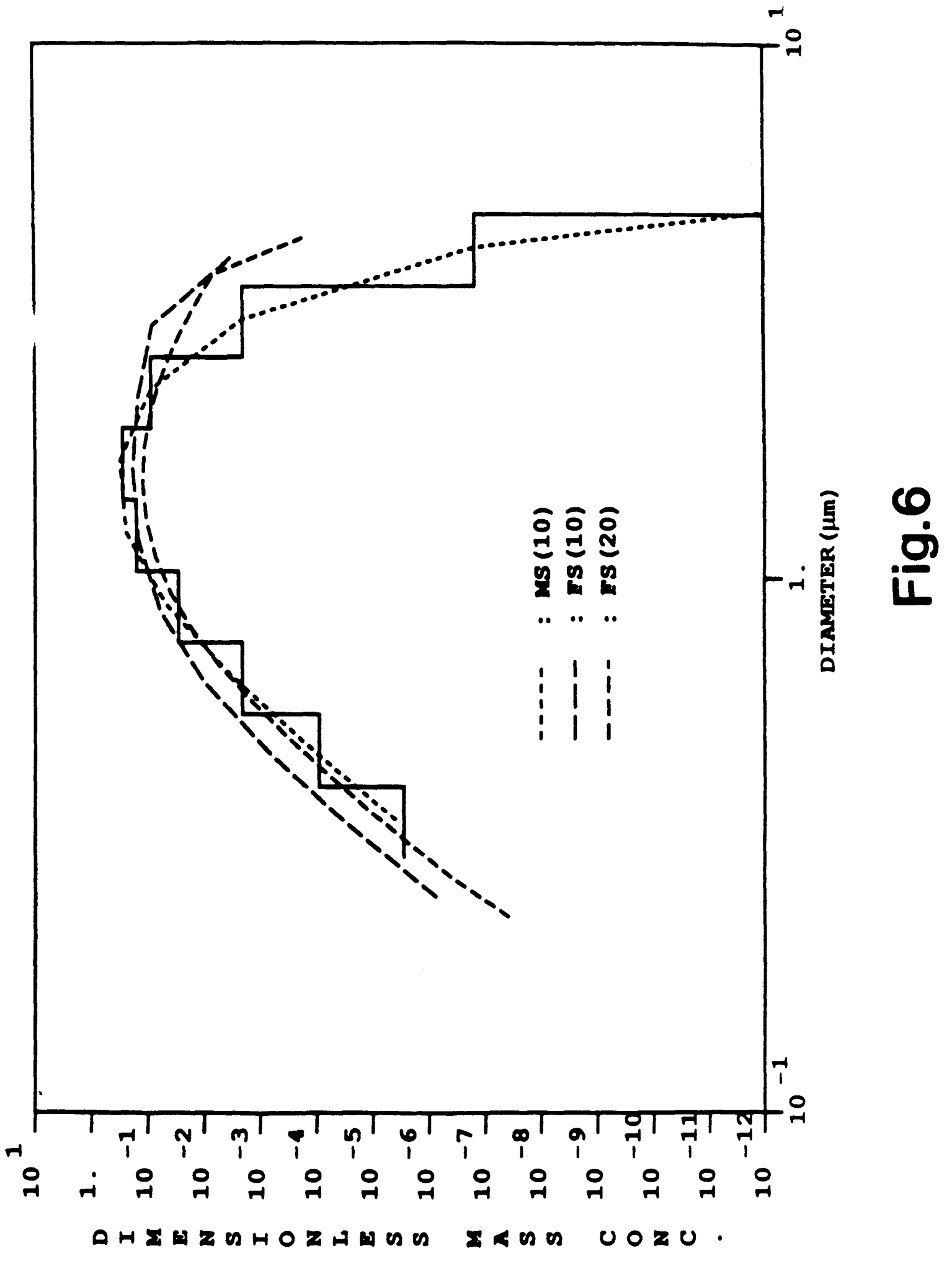


Chapter III

\section{SIMULATION OF \\ MULTICOMPONENT AEROSOL DYNAMICS}




\section{INTRODUCTION}

Multicomponent aerosols arise in diverse cases: in the atmosphere $(2,15,23$, $24)$; in indoor air (19); in material synthesis (8); and in nuclear reactor containment environments $(9,25)$. A multicomponent aerosol is characterized by a sizecomposition distribution function and that function is governed by the aerosol general dynamic equation (GDE) (13). The GDE includes terms that represent condensation (growth/evaporation), coagulation, source, nucleation, and removal processes. Numerically solving the full multicomponent aerosol GDE presents several computational challenges because of the inherently different mathematical nature of condensation and coagulation processes and the multidimensional nature of the size-composition distribution. Also particle size typically varies by three to five orders of magnitude in particle diameter or nine to fifteen orders of magnitude in particle mass, and the distribution itself frequently varies by several orders of magnitude.

Relatively few solutions of the multicomponent aerosol GDE exist, either analytical $(11,25)$ or numerical $(3,9,10,14,16,21,22,31,35)$. Presently two numerical methods are available for simulating multicomponent aerosol dynamics, the sectional method (14) and the moving finite element method (31). In the sectional method, the size distribution is divided into a finite number of sections, the size distribution in a section is assumed to be uniform, and the mean mass fractions of species of all particles in a section are assumed to be identical. While the assumption that the size distribution in a section is uniform itself introduces numerical errors (35), such errors exist even in higher order approximations, so this assumption is best viewed as a necessary mathematical approximation. The assumption that all particles in a given section have identical mean mass fractions, however, is advantageous because it eliminates the need to know the composition distributions in the sections to determine the component mass concentrations in the sections. Although a composition distribution in a section is permitted in the sectional method, it is neither calculated nor used in the sectional method simulation.

The sectional method with moving grids has several advantages over the fixed 
grid sectional method. In general, a numerical method based on the Lagrangian approach, the moving grid method, can be highly accurate and efficient for simulating condensation processes since there is no need to discretize the growth term. Furthermore, since sections can overlap each other, this method can simulate particles of the same size but different composition. Treating coagulation or sources, however, becomes difficult due to the deformation of the calculational domain that results when the sections are allowed to move (16). Thus, for aerosol dynamics consisting only of condensation a numerical method based on the Lagrangian approach is the preferred choice. Unfortunately, this approach loses its advantages when simulating simultaneous condensation and coagulation. There have been several efforts to extend the attributes of the Lagrangian approach $(20,36)$. One approach is to generate new grids or elements whenever they are needed. For a system of fast condensation and sources of small particles the number of new grids required can be quite large. Another approach is to remap or reinitialize the distribution based on the moving grids to fixed grids, but this remapping also introduces numerical errors. Tsang and Rao (31) extended the one-dimensional moving finite element method (FEM) along the characteristics $(32)$ to simulating aerosol condensation in two dimensions. While the method is highly accurate, it is only applicable to cases in which all components grow, not to general condensation processes, due to the loss of grid points (29). Also, the method is unsuitable for simultaneous processes, such as condensation, coagulation, and sources due to the deformation of the computational domain.

Condensation is a number conserving process (as long as particles do not completely evaporate), while coagulation is a mass conserving process. Since the numbercomposition distribution cannot be negative, negative values are unacceptable. $\mathrm{Nu}$ merical methods that conserve total number concentration during condensation (for evaporation number conservation may be applied to particles larger than the critical size) and conserve total mass concentration during coagulation are preferred. Non-negativity and proper conservation of number or mass are especially important characteristics a numerical method should possess since their absence results in phys- 
ically unacceptable solutions. Since we are interested in solution of the full GDE with multiple processes, we seek a method that is able to simulate the spectrum of processes that can occur. Numerical methods that perform well for simulating one process but cannot be used with other methods to simulate simultaneous processes are not suitable. If one has a process of pure condensation, then a Lagrangian method is known to be best. To develop a robust method for simultaneous coagulation and condensation, one must inevitably compromise accuracy in order to accommodate the simultaneous processes.

Our goals in this work are twofold: (1) to develop a technique to numerically solve the multicomponent aerosol GDE directly without introducing any physical assumptions about the nature of the size-composition distribution; and (2) to develop a technique applicable for simultaneous coagulation and condensation. Three different cases, pure condensation, pure coagulation, and simultaneous condensation and coagulation will be studied numerically. Model problems of multicomponent aerosol dynamics for which analytical solutions have been obtained are solved numerically to evaluate the accuracy of the numerical scheme. The effects of choice of condensation simulation scheme, rate form, and boundary condition are discussed. In addition, the direct numerical solution is compared to that of the sectional method. Finally, several aspects of the direct solution, including its applicability and limitations, are discussed.

\section{MULTICOMPONENT AEROSOL GENERAL DYNAMIC EQUATION}

The multicomponent aerosol general dynamic equation (GDE) governing the sizecomposition distribution function, $n(\mathbf{m}, t)$, is

$$
\frac{\partial n(\mathbf{m}, t)}{\partial t}+\sum_{i=1}^{s} \frac{\partial}{\partial m_{i}}\left[I_{i}(\mathbf{m}, t) n(\mathbf{m}, t)\right]
$$




$$
\begin{aligned}
& =\frac{1}{2} \int_{0}^{m_{1}} \cdots \int_{0}^{m_{s}} \beta(\mathbf{u}, \mathbf{m}-\mathbf{u}) n(\mathbf{m}-\mathbf{u}, t) n(\mathbf{u}, t) d \mathbf{u} \\
& -n(\mathbf{m}, t) \int_{0}^{\infty} \cdots \int_{0}^{\infty} \beta(\mathbf{u}, \mathbf{m}) n(\mathbf{u}, t) d \mathbf{u} \\
& +R[\mathbf{m}, t, n(\mathbf{m}, t)]+S[\mathbf{m}, t, n(\mathbf{m}, t)]
\end{aligned}
$$

where $m_{i}$ is the mass of the $i$-th component in a particle, and $\mathbf{m}$ is a vector of compositions $\left(m_{1}, \ldots, m_{s}\right)$, where $s$ is the total number of components. $n(\mathbf{m}, t) d \mathbf{m}$ is the number of particles having mass of component $i$, in the range $\left[m_{i}, m_{i}+d m_{i}\right]$ at time $t . I_{i}$ is the time rate change of the mass concentration of the $i$-th component from condensation process. $\beta(\mathbf{u}, \mathbf{m})=\beta(\mathbf{m}, \mathbf{u})$ is the binary coagulation coefficient. $R$ and $S$ are removal and source terms, respectively. The initial condition is

$$
n(\mathbf{m}, 0)=n_{o}(\mathbf{m})
$$

One possible boundary condition is that there is no particles below the critical size, $\mathrm{m}^{*}$

$$
n\left(\mathbf{m}^{*}, t\right)=0
$$

Since the range of values of $\mathrm{m}$ is quite large (usually several orders of magnitude), a logarithmic transformation is useful $(12,14)$,

$$
x_{i}=\ln \left(\frac{m_{i}}{M_{o}}\right) \quad i=1, \ldots, s
$$

where $M_{o}$ is the mean mass of a particle in the initial distribution,

$$
M_{\circ}=\sum_{i=1}^{s} \frac{1}{N_{o}} \int_{0}^{\infty} \ldots \int_{0}^{\infty} u_{i} n_{o}(\mathbf{u}) d \mathbf{u}
$$

where $N_{o}$ is the total number concentration of the initial distribution. Also by applying a property of the density function (24),

$$
n(\mathbf{m}) d \mathbf{m}=n(\mathbf{x}) d \mathbf{x}
$$

the resultant transformed GDE in a computational domain $\left[\mathbf{x}^{\alpha}, \mathbf{x}^{\beta}\right]$ is,

$$
\frac{\partial n(\mathbf{x}, t)}{\partial t}+\sum_{i=1}^{s} \frac{\partial}{\partial x_{i}}\left[I_{i}(\mathbf{x}, t) n(\mathbf{x}, t)\right]
$$




$$
\begin{aligned}
& =\frac{1}{2} \int_{x_{1}^{\alpha}}^{x_{1}} \cdots \int_{x_{s}^{\alpha}}^{x_{s}} \beta(\mathbf{q}, \mathbf{y}) n(\mathbf{q}, t) n(\mathbf{y}, t) \prod_{i=1}^{s}\left(\frac{e^{x_{i}}}{e^{x_{i}}-e^{y_{i}}}\right) d \mathbf{y} \\
& -n(\mathbf{x}, t) \int_{x_{1}^{\alpha}}^{x_{1}^{\beta}} \cdots \int_{x_{s}^{\alpha}}^{x_{s}^{\beta}} \beta(\mathbf{x}, \mathbf{y}) n(\mathbf{y}, t) d \mathbf{y} \\
& +R[\mathbf{x}, t, n(\mathbf{x}, t)]+S[\mathbf{x}, t, n(\mathbf{x}, t)]
\end{aligned}
$$

where $q$ is given by,

$$
\begin{aligned}
q_{i} & =\ln \left(\frac{m_{i}}{M_{o}}-\frac{u_{i}}{M_{o}}\right) \\
& =\ln \left(\exp \left[x_{i}\right]-\exp \left[y_{i}\right]\right) \quad i=1, \ldots, s .
\end{aligned}
$$

The transformed initial condition is,

$$
\begin{aligned}
n(\mathbf{x}, 0) & =n_{o}(\mathbf{x}) \\
& =n_{o}(\mathbf{m}) \prod_{i=1}^{s} m_{i}
\end{aligned}
$$

and an appropriate boundary condition is required either at $x_{i}=x_{i}^{\alpha}$, if the $i$-th component grows, or at $x_{i}=x_{i}^{\beta}$, if the $i$-th component evaporates. Removal and source terms are not considered here. To include these terms in a numerical scheme is not difficult once the numerical scheme treating condensation and coagulation is established.

If the characteristic times of all or several processes are the same order of magnitude, the processes have to be calculated simultaneously. But, if the characteristic time of one process is significantly larger than those of other processes, that process can be regarded as in a pseudo-steady state with respect to other processes. The problem of characteristic time difference is well exemplified by the following: Consider the vapor phase synthesis of ceramic particles at high supersaturation ratio. At first, particles are rapidly produced by nucleation and other processes are negligible. After the initial particle burst, both nucleation and condensation will occur, competing for the available condensible gas phase species. During this, coagulation can be neglected. After the condensible gas phase species is depleted and the number concentration of particles increases, coagulation and deposition will be important while nucleation and condensation are negligible. 


\section{NUMERICAL METHODS FOR AEROSOL DYNAMICS}

\subsection{Simulation of the Condensation Term in the Multicomponent GDE}

The evolution of an aerosol number-composition distribution as the result of condensation processes is described by a first-order hyperbolic partial differential equation, of the form that also describes general advection processes. The difficulties of solving this equation numerically both accurately and efficiently are well known $(17,20,36)$. In particular, discrete approximation of the spatial derivatives of the hyperbolic equation may lead to significant numerical dispersion and diffusion errors (16). Another specific problem associated with condensation is that the rates may vary quite widely, often over several orders of magnitude. Therefore, a numerical scheme that works well for other advection process may not be suitable for aerosol condensation processes.

A numerical scheme for the condensation process should possess following properties: (1) It should keep the distribution positive definite; (2) It should conserve the total number concentration; (3) It should be accurate; and (4) It should be efficient. If condensation only is occurring, then an appropriate Lagrangian method can satisfy the above four conditions $(10,16,31)$. However, as we noted earlier, a numerical method based on the Lagrangian approach is not suitable for simultaneous condensation and coagulation, and we seek here a method that can be used as part of a numerical approach to solve the fully multicomponent GDE.

Two numerical methods, the repeated upwind difference method (RUD) $(26,27)$ and the Taylor-Galerkin method (TGFEM) (7) are chosen here to treat condensation term in the GDE. The RUD was developed to reduce excessive numerical diffusion errors associated with the first-order upwind difference method. The RUD satisfies properties 1 and 2 but is only second-order accurate. Furthermore, the RUD is known to smear sharp peaks in the distribution $(5,28,29)$. The TGFEM was developed to reduce the numerical dispersion errors associated with the Galerkin method. While the TGFEM satisfies properties 3 and 4, it does not satisfy properties 1 and 2 . 


\subsubsection{The Repeated Upwind Difference Method (RUD)}

The first-order accurate upwind difference method for numerical evaluation of Eq. [7] without coagulation can be written in the form

$n_{i}^{l+1}=n_{i}^{l}-\sum_{k=1}^{s}\left[F^{k}\left(n_{i}^{l}, n_{i+e_{k}}^{l}, I_{i+(1 / 2) e_{k}}^{k}\right)-F^{k}\left(n_{i-e_{k}}^{l}, n_{i}^{l}, I_{i-(1 / 2) e_{k}}^{k}\right)\right]$

where $n_{i}^{l}$ is a numerical approximation of the solution of Eq. [7] (without coagulation), defined at time $l \Delta t$ and size $\left(i^{1} \Delta x^{1}, \ldots, i^{s} \Delta x^{s}\right)$, and where $e_{k}$ is a unity vector in the $k$-th component, $I_{i+(1 / 2) e_{k}}^{k}$ is the $k$-th component condensation rate at the midpoints of the size domain. $F^{k}$ is the condensation flux of the $k$-th component evaluated at the same midpoints as the $k$-th component condensation rate and is defined as follows:

$F^{k}\left(n_{i}, n_{i+e_{k}}, I\right) \equiv\left[(I+|I|) n_{i}+(I-|I|) n_{i+e_{k}}\right] \frac{\Delta t}{2 \Delta x^{k}}$.

The first-order upwind difference methods have several desirable properties; they maintain positivity, are stable and free from numerical dispersion errors, conserve total number concentration during the simulation of condensation processes, are easy to formulate and require less computing time than higher-order methods. A drawback is that these methods suffer from excessive numerical diffusion errors. The rate of the excessive diffusion in the case of a constant condensation rate may be estimated by expanding $n_{i}^{l+1}, n_{i+e_{k}}^{l}$, and $n_{i-e_{k}}^{l}$ in a second-order Taylor series about the point $\left(t^{l}, x_{i}\right)$. Doing so, the numerical method may be expressed as

$$
\begin{aligned}
\left.\frac{\partial n}{\partial t}\right|_{i} ^{l}= & -\left.\sum_{k=1}^{s} \frac{\partial}{\partial x^{k}}\left(I^{k} n\right)\right|_{i} ^{l}+\sum_{k=1}^{s} \frac{\partial}{\partial x^{k}}\left(0.5\left[\left|I^{k}\right| \Delta x^{k}-\Delta t\left(I^{k}\right)^{2}\right] \frac{\partial n}{\partial x^{k}}\right. \\
& \left.-\sum_{j=1, \neq k}^{s} 0.5 \Delta t I^{k} I^{j} \frac{\partial n}{\partial x^{j}}\right)\left.\right|_{i} ^{l} .
\end{aligned}
$$

The first term on the right hand side (RHS) of above equation represents the original condensation term, while the second term on the RHS represents the induced excessive numerical diffusion that should be removed to get an exact solution.

The repeated upwind difference method developed by Smolarkiewicz $(26,27)$ is selected as the one of the two numerical schemes. The strengths of this scheme are: 
(1) It has the desirable properties of the upwind difference methods; (2) It is a fully multidimensional method and can be used without adopting the spatial fractional time steps scheme; (3) It is easy to formulate; and (4) In comparisons with the SHASTA method, a type of flux-corrected transport (FTC) method (4) that has been widely tested and used, for the simulation of single component aerosol dynamics with simultaneous coagulation and condensation, the repeated upwind difference method has been found to be both more accurate and more computationally efficient than the SHASTA method by the authors.

The basic idea of the RUD is to subtract the excessively diffused amount from the transported quantity and add that amount back to the original grid cell by defining a so-called antidiffusion velocity and using the first-order upwind difference method, repeatedly if necessary. The reversion of the numerical diffusion can be written as,

$$
\frac{\partial n}{\partial t}=-\sum_{k=1}^{s} \frac{\partial}{\partial x^{k}}\left(\tilde{I}^{k} n\right)
$$

in the next corrective step. $\tilde{I}^{k}$ is the antidiffusive rates defined as follows:

$$
\begin{aligned}
\tilde{I}^{k} & =0.5\left[\left|I^{k}\right| \Delta x^{k}-\Delta t\left(I^{k}\right)^{2}\right] \frac{1}{n} \frac{\partial n}{\partial x^{k}}-\sum_{j=1, \neq k}^{s} 0.5 \Delta t I^{k} I^{j} \frac{1}{n} \frac{\partial n}{\partial x^{j}} \quad \text { if } \quad n>0 \\
& =0 \quad \text { if } \quad n=0
\end{aligned}
$$

which, in fact, is the second term on the RHS of Eq. [12] if $n>0$. Based on this concept, the corrective step (the repeated upwind difference step) is

$n_{i}^{l+1}=n_{i}^{*}-\sum_{k=1}^{s}\left[F^{k}\left(n_{i}^{*}, n_{i+e_{k}}^{*}, \tilde{I}_{i+(1 / 2) e_{k}}^{k}\right)-F^{k}\left(n_{i-e_{k}}^{*}, n_{i}^{*}, \tilde{I}_{i-(1 / 2) e_{k}}^{k}\right)\right]$

where $n^{*}$ is given by the RHS of Eq. [10] and

$$
\begin{aligned}
\tilde{I}_{i+(1 / 2) e_{k}}^{k}= & {\left[\left|I_{i+(1 / 2) e_{k}}^{k}\right| \Delta x^{k}-\Delta t\left(I_{i+(1 / 2) e_{k}}^{k}\right)^{2}\right] \frac{n_{i+e_{k}}^{*}-n_{i}^{*}}{\left(n_{i+e_{k}}^{*}+n_{i}^{*}+\varepsilon\right) \Delta x^{k}} } \\
& -\sum_{j=1, \neq k}^{s} 0.5 \Delta t I_{i+(1 / 2) e_{k}}^{k} \bar{I}_{i+(1 / 2) e_{k}}^{j} \\
& \times \frac{n_{i+e_{k}+e_{j}}^{*}+n_{i+e_{j}}^{*}-n_{i+e_{k}-e_{j}}^{*}-n_{i-e_{j}}^{*}}{\left(n_{i+e_{k}+e_{j}}^{*}+n_{i+e_{j}}^{*}+n_{i+e_{k}-e_{j}}^{*}+n_{i-e_{j}}^{*}+\varepsilon\right) \Delta x^{k}}
\end{aligned}
$$


where

$\bar{I}_{i+(1 / 2) e_{k}}^{j}=0.25\left(I_{i+e_{k}+(1 / 2) e_{j}}^{j}+I_{i+(1 / 2) e_{j}}^{j}+I_{i+e_{k}-(1 / 2) e_{j}}^{j}+I_{i-(1 / 2) e_{j}}^{j}\right)$

and $\varepsilon$ is a small value, e.g., $10^{-15}$, to ensure $\tilde{I}^{k}=0$ when all functional values of $n$ of Eq. [16] are equal to zero. The corrective step, Eq. [15], is again the upwind difference scheme and contains numerical diffusion errors that can be reversed by the next corrective step. The number of corrective steps, denoted by $\operatorname{IORD}$, is optional. Note that $I O R D=1$ implies only the original upwind difference method. Smolarkiewicz (27) reported that using $I O R D>4$ only negligibly improves the accuracy of the numerical solution over using $\operatorname{IORD}=4$. If the condensation rates are in divergent form, the above corrective formula assumes the form

$$
\begin{aligned}
\tilde{I}_{i+(1 / 2) e_{k}}^{k}=\ldots- & 0.25 \Delta t I_{i+(1 / 2) e_{k}}^{k}\left(I_{i+(3 / 2) e_{k}}^{k}-I_{i-(1 / 2) e_{k}}^{k}\right) / \Delta x^{k} \\
- & 0.25 \Delta t I_{i+(1 / 2) e_{k}}^{k} \sum_{j=1, \neq k}^{s}\left(I_{i+(1 / 2) e_{j}}^{j}+I_{i+(1 / 2) e_{j}+e_{k}}^{j}\right. \\
& \left.-I_{i-(1 / 2) e_{j}}^{j}+I_{i-(1 / 2) e_{j}+e_{k}}^{j}\right) / \Delta x^{j}
\end{aligned}
$$

where the terms omitted are the same as those in Eq. [16]. The method is secondorder accurate in both time and space (27).

The repeated upwind difference method is not without drawbacks. First, the method is generally less accurate than other methods, such as the Galerkin methods, for simulating advection, or the moving FEM for simulating condensation of single and two-component aerosol systems $(5,30,31)$. However, both the Galerkin method and the moving FEM do not guarantee non-negativity and number conservation which are deemed essential in aerosol simulation. And as mentioned earlier, a numerical method based on the Lagrangian approach is not suitable for simultaneous condensation and coagulation. Therefore, while the RUD is not as accurate as one might wish, it has reasonable accuracy and several desirable properties that are essential for the simulation of multicomponent aerosol dynamics. The stability of the RUD has been proved only up to three dimensions (27). For the simulation of a multicomponent aerosol that may consist of more than three components, stability is 
of course essential. We will subsequently demonstrate by numerical experiments the stability of this method for cases of more than three dimensions.

Computing time increases with the number of repeated computation steps. Smolarkiewicz (27) reported that increasing the number of iteration steps enhances the accuracy of the scheme up to $\operatorname{IOR} D=4$, but the computing time required for four iterations is similar to that of a FCT method. Smolarkiewicz $(26,27)$ suggested a compromise between accuracy and computing time by increasing the antidiffusive velocity, $\tilde{I}^{k}$, by multiplying by a factor $S c$,

$$
\tilde{I}^{k}=\left(\tilde{I}^{k}\right)_{E q \cdot[16] \operatorname{or}[18]} \times S c
$$

He reported that a small increase of $S c$ over unity $($ e.g., $S c=1.06)$ significantly improves the solution for the IORD $=2$ scheme (one iteration). We have carried out numerical experiments to choose the optimal values of two parameters, the number of iterations, $I O R D$, and the scale factor, $S c$, and these will be described subsequently.

\subsubsection{The Taylor-Galerkin Method (TGFEM)}

In the Galerkin method, a distribution is approximated by a finite series

$$
n(\cdot) \approx \hat{n}(\cdot)=\sum_{j=1}^{m} n_{j} \phi_{j}(\cdot)
$$

where $n_{j}$ is an undetermined coefficient and $\phi_{j}(\cdot)$ is the basis function or shape function. Let the governing equation be represented by

$\operatorname{Ln}(\cdot)-f=0$

where $\mathrm{L}$ is an operator and $f$ is an arbitrary function. But the approximation, $\hat{n}$ does not satisfy the above governing equation exactly, so there exists so-called the residual error, $R(\cdot)$,

$$
R(\cdot)=\mathrm{L} \hat{n}(\cdot)-f .
$$


A class of numerical schemes, called the method of weighted residuals, has been developed to minimize the residual error by selecting the undetermined coefficients $n_{j}$ that satisfy the following integral relation;

$$
\int R(\cdot) w_{i}(\cdot) d V=0 \quad i=1,2, \ldots, m
$$

where $w_{i}(\cdot)$ is called the weighting function. In the Galerkin methods, the weighting function is chosen to be the basis function, so

$$
\int R(\cdot) \phi_{i}(\cdot) d V=0 \quad i=1,2, \ldots, m .
$$

If a piecewise linear function (a chapeau function) is used as the basis function then the Galerkin method is also called as the chapeau function method or the linear finite element method (17).

The Galerkin method has several advantages: (1) It is highly accurate; (2) It can retain a sharp peak distribution without smearing. But the method has the serious disadvantage of numerical dispersion errors. Numerical dispersion errors may shift the peak position and/or produce spurious oscillations in the numerical distribution that frequently can cause negative values in distribution (16).

There are some approaches to eliminate or at least reduce the undesirable nimerical dispersion errors. One approach is to add the upwind term to the condensation term (or in general term, on the advection term), so the numerical dispersion errors are suppressed by the numerical diffusion. Usually in the numerical schemes, the upwind effect is added to the weighting function so this kind of approach is called the asymmetric weighting function Galerkin method or the Petrov-Galerkin method (PGFEM) (17).

A new approach (7) was proposed in introducing the upwind effect into the Galerkin method in which the forward-time Taylor series expansion up to the thirdorder time derivative is applied to the governing equation and the second-order time derivative is replaced by a spatial form and the third-order term by a mixed spatialtemporal form. By doing so, the usual Galerkin method approach can be used, so this 
method is called the Taylor-Galerkin method (TGFEM). Depending on the original time discretization scheme, one can obtain the Euler TGFEM, the leap-frog TGFEM, or the Crank-Nicolson TGFEM (7). The Euler TGFEM with time splitting $(5,7)$ is chosen here as the one of the two condensation simulation schemes. It was shown as a method superior to the RUD and the PGFEM with high-degree weighting for simulating a rotating cosine hill by Chock (5). For a model problem presented by Donea (7) and Chock (5) the RUD predicted about $51 \%$ while the TGFEM predicted $105 \%$ of the peak value. It has no adjustable parameters while the RUD or the PGFEM has one or two. It is straightforward and easy to implement. But as with the other Galerkin methods, this method does not totally eliminate numerical dispersion errors. Thus, this method also does not guarantee non-negativity or number conservation even with a nonlinear filter (5), so the original form with the time splitting method is used (7).

\subsection{Simulation of the Coagulation Term in the Multicomponent GDE}

To represent the coagulation integrals accurately and efficiently is a demanding problem even in the case of a single component. Due to the logarithmic transformation, the numerical value of $q_{i}$ does not coincide with that at the grid points even if both values $x_{i}$ and $y_{i}$ as shown in Eq. [8] are at grids. The cubic spline method has been widely used for interpolation and integration. In single component coagulation systems, the cubic spline method has been used to calculate the coagulation terms (18). It is applicable in principle to multidimensional cases but rarely has been applied to cases of more than two dimensions (1). Furthermore, the computing time required to calculate coefficients of the third-order polynomial for each dimension is sufficiently excessive that it does not appear to be attractive to use the multidimensional cubic spline method to represent the multicomponent coagulation integrals.

Gaussian quadrature with a product rule is selected here to perform the multiple 
integrals. The Gaussian quadrature has been well proved and extensively tested (6, 28 ) and this method is already used in the multicomponent sectional method to evaluate double integrals (34).

The one-dimensional integration can be approximated by

$$
\int_{a}^{b} w(x) n(x) d x \approx \sum_{k=1}^{m} w_{k} n\left(x_{k}\right)
$$

If $w(x)$ is the non-negative weight function with corresponding orthonormal polynomials and ..: are the weights represented by the specified relation of the orthonormal polynomials, and $x_{k}$ 's are the zeros of the orthonormal polynomial satisfying, $a<x_{1}<\ldots<x_{m}<b$, then the above integration approximation is of Gaussian type (6). In this work, a Jacobi polynomial is used as the orthonormal polynomial in the transformed domain of $[0,1](33)$. The polynomial used here is

$$
p_{N}(x)=\left[x-g_{N}(N, a, b)\right] p_{N-1}(x)-h_{N}(N, a, b) p_{N-2}
$$

where $a=0$ if the boundary point $x=1$ is not included in the grid point, and $b=0$ if the boundary point $x=0$ is not included in the grid point. Also

$$
\begin{aligned}
& g_{1}=\frac{b+1}{a+b+2} \\
& g_{N}=\frac{1}{2}\left[1-\frac{a^{2}-b^{2}}{(2 N+a+b-1)^{2}-1}\right] \quad N>1 \\
& h_{1}=0 \quad \\
& h_{2}=\frac{(a+1)(b+1)}{(a+b+2)^{2}(a+b+3)} \\
& h_{N}=\frac{(N-1)(N+a-1)(N+b-1)(N+a+b-1)}{(2 N+a+b-1)(2 N+a+b-2)^{2}(2 N+a+b-3)} \quad N>2 .
\end{aligned}
$$

The weight function and the weights are

$$
\begin{aligned}
& w(x)=x^{b}(1-x)^{a} \\
& w_{k}=\int_{0}^{1} l_{k}(x)(1-x)^{a} x^{b} d x
\end{aligned}
$$

respectively. $l_{k}(x)$ is

$$
l_{k}(x)=\frac{p_{N}(x)}{\left(x-x_{k}\right) p^{(1)}\left(x_{i}\right)} .
$$


Since the grids do not contain boundaries, both $a$ and $b$ are set to zero.

In the multidimensional case, a product rule of a one-dimensional scheme is usually used (6). A two-dimensional integration product rule is shown as an example,

$$
\int_{a}^{b} d x \int_{c(x)}^{d(x)} n(x, y) d y \approx \sum_{k=1}^{m} w_{k} \sum_{j=1}^{p} v_{j k} n\left(x_{k}, y_{j}\right)
$$

The log-linear approximation method is used for the interpolation of each component. For example, the two-dimensional linear interpolation formula is,

$$
\begin{aligned}
\ln n^{*}= & \left(\frac{\Delta x_{i}-h_{i}}{\Delta x_{i}}\right)\left(\frac{\Delta y_{j}-h_{j}}{\Delta y_{j}}\right) \ln n\left(x_{i}, y_{j}\right) \\
+ & \left(\frac{\Delta x_{i}-h_{i}}{\Delta x_{i}}\right)\left(\frac{h_{j}}{\Delta y_{j}}\right) \ln n\left(x_{i}, y_{j+1}\right) \\
+ & \left(\frac{h_{i}}{\Delta x_{i}}\right)\left(\frac{\Delta y_{j}-h_{j}}{\Delta y_{j}}\right) \ln n\left(x_{i+1}, y_{j}\right) \\
+ & \left(\frac{h_{i}}{\Delta x_{i}}\right)\left(\frac{h_{j}}{\Delta y_{j}}\right) \ln n\left(x_{i+1}, y_{j+1}\right)
\end{aligned}
$$

where $n^{*}$ is the approximated functional value at $\left(x_{i}+h_{i}, y_{j}+h_{j}\right)$ and $\Delta x_{i}$ and $\Delta y_{j}$ are the grid spacings of the $i$-th and $j$-th component directions, respectively. Though less accurate than higher order interpolation methods, this selection represents a compromise in computing time. As shown later, even the linear approximation method for the coagulation integrals consumes the largest portion of the total computing time of the full GDE.

\subsection{Simulation of Simultaneous Condensation and Coagulation.}

The operator splitting method (20) is applied for simultaneous condensation and coagulation. In the operator splitting method, if the values of the size-composition distribution do not change too rapidly over a time step as a result of any of the individual processes, one can add the changes occurring as a result of the individual processes to calculate the total change in $n$,

$$
\left.n\right|_{t+\Delta t}=\left.n\right|_{t}+\left.\Delta n\right|_{\text {condensation }}+\left.\Delta n\right|_{\text {coagulation }}
$$


The validity of the above relation is determined by the ratios of $\left.\Delta n\right|_{\text {condensation }}$ and $\left.\Delta n\right|_{\text {coagulation }}$ to $\left.n\right|_{t}$; both $\left.\Delta n\right|_{\text {condensation }} /\left.n\right|_{t}$ and $\left.\Delta n\right|_{\text {coagulation }} /\left.n\right|_{t}$ must be much smaller than the order of one. If the characteristic times of condensation and coagulation differ, the choice of the computing time step $\Delta t$ is determined by the smaller characteristic time. We have checked the validity of this method by changing the values of $\Delta t$ and calculating $\left(\Delta n /\left.n\right|_{t}\right)$ 's and the results presented in Section 4.1. confirm the validity of using the time splitting method.

\section{NUMERICAL SOLUTION OF}

\section{MULTICOMPONENT AEROSOL DYNAMICS}

In this section the proposed numerical scheme is applied to model problems. Three problems are studied, pure condensation, pure coagulation, and simultaneous condensation and coagulation. Systems of up to four components have been simulated and compared to the analytical solutions. Also numerical solutions of the three-component system are compared to the those from the sectional method. The cases studied are summarized in Table I. The model problems having analytical solutions are employed so as to evaluate the accuracy of the numerical scheme.

\subsection{Exact Solutions for Special Cases}

For reasonably complicated expressions for condensation rates and coagulation coefficient, it is impossible to obtain analytical solutions for simultaneous processes. To obtain the analytical solutions for simultaneous processes, linear condensation rates and constant coagulation coefficient are assumed. These are

$$
\begin{aligned}
& I_{i}=\alpha_{i} m_{i} \quad i=1, \ldots, s \\
& \beta(m, u)=\beta_{0} .
\end{aligned}
$$


Note that if the value of $\alpha_{i}$ is negative the $i$-th component evaporates. Though simplified, linear condensation rates such as in Eq. [35] represent a class of condensation mechanisms arising from volume reaction-controlled gas-to-particle conversion (24). The rate of coagulation is dependent on the sizes of the two particles irrespective of the mechanism except for the early stage of coagulation of a monodisperse aerosols in the continuous regime (24), so a constant coagulation coefficient is an idealized simplification needed to obtain the analytical solutions.

The pure condensation process with one-third order rate is also studies to check the performance of the numerical methods for various condensation rate formulae.

$$
I_{i}=\alpha_{i} \sum_{j=1}^{s}\left(m_{j}\right)^{1 / 3} \quad i=1, \ldots, s .
$$

These rates represent a class of condensation mechanisms arising from gas diffusioncontrolled gas-to-particle conversion, also known as the Maxwell relation (24).

An exponential initial distribution is considered:

$$
n_{o}(m)=N_{o} \prod_{i=1}^{s} \frac{1}{m_{i o}} \exp \left[-\frac{m_{i}}{m_{i o}}\right]
$$

where $N_{o}$ and $m_{i o}$ are the total number concentration and the mean mass of the $i$-th component in a particle, respectively, and are given by

$$
\begin{aligned}
& N_{o}=\int_{0}^{\infty} \ldots \int_{0}^{\infty} n_{o}(m) d m \\
& m_{i o}=\frac{1}{N_{o}} \int_{0}^{\infty} \cdots \int_{0}^{\infty} m_{i} n_{o}(m) d m .
\end{aligned}
$$

The exact solution of Eq. [7] for pure condensation processes subject to Eqs. [35] and [38] is obtained by the method of characteristics and the result is,

$$
n(m, t)=N_{o} \prod_{i=1}^{s} \frac{1}{m_{i o}} \exp \left[-\frac{m_{i}}{m_{i o}} \epsilon^{-\Lambda_{i} \tau}-\Lambda_{i} \tau\right]
$$

where $\Lambda_{i}$ is dimensionless condensation rate, $\Lambda_{i}=\frac{\alpha_{i}}{\alpha_{o}}$, and $\tau$ is the dimensionless time defined as, $\tau=t \alpha_{o}$, where $\alpha_{o}$ is an arbitrary condensation rate, but usually $\sum_{j=1}^{s}\left|\alpha_{j}\right|$. 
The exact solution of Eq. [7] for pure condensation processes subject to Eqs. [37] and [38] is also obtained by the method of characteristics and the result is,

$$
\begin{aligned}
n(m, t)= & N_{o} \prod_{i=1}^{s} \frac{1}{m_{i o}} \exp \left[-\frac{1}{m_{i o}}\left(m_{i}-\frac{\alpha_{i}}{\sum_{j=1}^{s} \alpha_{j}}\left(\sum_{j=1}^{s} m_{j}-\left[\left(\sum_{j=1}^{s} m_{j}\right)^{2 / 3}-\frac{2}{3} \sum_{j=1}^{s} \alpha_{j} t\right]^{3 / 2}\right)\right)\right] \\
& \times \frac{\left[\left(\sum_{j=1}^{s} m_{j}\right)^{2 / 3}-\frac{2}{3} \sum_{j=1}^{s} \alpha_{j} t\right]^{1 / 2}}{\left(\sum_{j=1}^{s} m_{j}\right)^{1 / 3}} .
\end{aligned}
$$

The exact solution of Eq. [7] for simultaneous condensation and coagulation processes subject to Eqs. [35]-[36], and [38] was obtained by Gelbard and Seinfeld (11),

$$
\begin{aligned}
n(m, \tau)= & \frac{4 N_{o}}{(\tau+2)^{2}} \prod_{i=1}^{s} \frac{1}{m_{i o}} \exp \left[-\frac{m_{i}}{m_{i o}} e^{-\Lambda_{i} \tau}-\Lambda_{i} \tau\right] \\
& \times \sum_{k=0}^{\infty} \frac{1}{(k !)^{s}}\left[\frac{\tau}{\tau+2} \prod_{j=1}^{s} \frac{m_{j}}{m_{j o}} e^{-\Lambda_{j} \tau}\right]^{k}
\end{aligned}
$$

where $\tau$ is the dimensionless time and is defined with a characteristic coagulation time, $\tau=\beta_{o} N_{o} t$, and $\Lambda_{i}$ is the ratio of the characteristic time for coagulation to the characteristic time for condensation of the $i$-th component of a particle, $\Lambda_{i}=\frac{\alpha_{i}}{\beta_{o} N_{o}}$. The analytical solution for pure coagulation is a special case of the above equation with all $\Lambda_{i}=0$.

The initial size-composition distribution is characterized by the initial total number concentration $\left(N_{\circ}\right)$ of $1 \times 10^{6} \mathrm{~cm}^{-3}$, mean particle mass $\left(M_{o}\right)$ of $5.2 \times 10^{-16} \mathrm{~g}$, which is equivalent to the mean mass diameter of $0.1 \mu \mathrm{m}$ if the particle density is $1 \mathrm{gcm}^{-3}$. The coagulation rate is $1 \times 10^{-7} \mathrm{~cm}^{3} \mathrm{~s}^{-1}$ and the condensation rate coefficients are $1 \times 10^{-1} \mathrm{~s}^{-1}$ for Eq. [35] and $1 \times 10^{-10} \mathrm{~g}^{2 / 3} \mathrm{~s}^{-1}$ for Eq. [37]. The dimensionless physical parameters, $m_{i o}$ and $\Lambda_{i}$, used in the test cases for up to four components are given in Table II. These parameters represent a system of aerosol components of similar initial mass concentrations and similar condensation rates.

To compare the numerical and analytical solutions quantitatively, two errors are defined: (1) The median relative error is that value of the relative error below which one half of the values of relative errors at all grid points and for all components lie; and (2) The maximum relative error is the maximum value of relative error over all 
grid points and for all components. These errors are defined as:

$$
\begin{aligned}
& \text { Median Relative Error } \\
& =\operatorname{Median}\left[\frac{\operatorname{abs}\left(\left.n\left(m_{j k}, t\right)\right|_{\text {analytical }}-\left.n\left(m_{j k}, t\right)\right|_{\text {numerical }}\right)}{\left.n\left(m_{j k}, t\right)\right|_{\text {analytical }}} \times 100\right] \\
& \quad j=1, \ldots, s \quad k=1, \ldots, n s_{j} \\
& \text { Maximum Relative Error } \\
& =\operatorname{Max}\left[\frac{\operatorname{abs}\left(\left.n\left(m_{j k}, t\right)\right|_{\text {analytical }}-\left.n\left(m_{j k}, t\right)\right|_{\text {numerical }}\right)}{\left.n\left(m_{j k}, t\right)\right|_{\text {analytical }}} \times 100\right] \\
& \quad j=1, \ldots, s \quad k=1, \ldots, n s_{j}
\end{aligned}
$$

where $j$ stands for the $j$-th component and $n s_{j}$ is the number of grids of the $j$-th component. The reason for using the relative error as the measure of accuracy is that the size-composition distribution varies by several orders of magnitude. Among the two measures of errors, the median relative error is indicative of the overall performance of the numerical method, while the maximum relative error tends to give the error only at the steepest portion of the profile. Also the changes of the total number and mass concentrations of the analytical and numerical solutions are obtained and compared to check the conservation performance of the numerical technique.

The calculational parameters summarized in Table III are employed. In dimensional terms, for example, the computational domain is $[0.0046 \mu \mathrm{m}, 0.27 \mu \mathrm{m}]$ for a single-component system and $[0.01 \mu \mathrm{m}, 0.22 \mu \mathrm{m}]$ for a two-component system. An equally spaced grid system is used. In most cases, 21 grids per component are employed, but for the four-component system, due to excessive computing time and memory requirements, the number of grids per component is reduced to 13 for pure condensation and to 7 for the cases including coagulation. The computational domain for the four-component system including coagulation is also reduced to account for the reduction of the number of grids. Also the computational domain of three-component system including coagulation is reduced without the change of the number of grids to study the effect of the number of grids. The changes of the analytical total number and mass concentrations have been calculated in order to check the performance of 
the numerical method. A forward Euler method is used for the time integration of the coagulation portion of the equation. Since a linear interpolation scheme is used, a higher order time integration method is unnecessary. A five-point Gaussian quadrature is employed. The choice of the number of quadrature points will be addressed later.

The numerical results to be presented here are those after one characteristic time. For the given conditions, the numerical values of the characteristic times are $10 \mathrm{~s}$ for condensation with linear rates, $7 \mathrm{~s}$ for condensation with one-third order rates, and $10 \mathrm{~s}$ for coagulation. The dimensionless time step is chosen as 0.01 except in the cases 4-6, and 9 in which cases the dimensionless time step is chosen as $2 \times 10^{-6}$ due to the Courant number restriction. The changes of the size distribution at each time step are generally within $2 \%$. A SUN386i and a SPARC station were used. When comparing computing times, results from the same computer were compared.

In calculating the numerical solution, the analytical expression is used for the inflow boundary condition except in cases 4-6, and 9 where a numerical boundary condition is used to eliminate the effect of the boundary condition on the performance of the numerical scheme. Also, to calculate the antidiffusive velocity in the repeated upwind difference method, a boundary condition at the outflow boundary is needed. Good results are obtained with a logarithmic-linear extrapolation, $w_{i, 1}=w_{i, 2}^{2} / w_{i, 3}$ for a two-component system, for the lower boundary and a symmetry condition, $w_{i, N 1}=w_{i, N-1}$, for the upper boundary. Usually the values at the boundary are much smaller than the peak values. As will be shown later, numerical solutions with this numerical boundary condition are in good agreement with those obtained with the analytical boundary condition.

\subsection{Pure Condensation}

We remind the reader that if one knows a priori that only condensation is occur- 
ring, then an appropriate Lagrangian method should be selected. In this subsection we will test the methods considered here for treating the condensation term in the full GDE, which, as described above, are not Lagrangian methods. The numerical tests to be presented are necessarily a severe test of the condensation portion of the overall method.

In this subsection, several cases of condensation are considered:

1. Linear rates.

- Case 1: Pure growth of up to a four-component system.

- Case 2: Pure evaporation of a two-component system.

- Case 3: Mixed growth/evaporation of a two-component system, one component evaporates and the other grows.

2. One-third order rates.

- Case 4: Pure growth of a two-component system.

- Case 5: Pure evaporation of a two-component system.

- Case 6: Mixed growth/evaporation of a two-component system, one component evaporates and the other grows.

While the second to the sixth cases are simulated only for a two-component system, they can readily be extended to a general multicomponent system. Also the effects of the choice of the two parameters of the RUD are studied. The TGFEM is tested for cases 1 and 4 .

Figure 1 shows the initial size-composition distribution assumed for a two-component system. Note that the distribution is dimensionless, the number distribution divided by the total number concentration. Figure 2 shows the analytical and numerical sizecomposition distributions for case 1, growth of a two-component system with linear condensation rates by the RUD. The effect of growth is to shift the size-composition 
distribution to larger sizes according to the characteristics, in this case linear condensation rates, of each component. The result of the TGFEM are indistinguishable from those by the RUD and no graphical presentation is therefore given.

Table IV gives the computing times and the numerical errors associated with case 1 for the both numerical methods. It is demonstrated that the RUD can be applied to more than three-dimensional cases. The overall performance of the RUD can be visualized from Figure 2 and the median relative errors are seen to be acceptable for situations of this type (Table IV). For example, the median relative error of the firstorder upwind difference method for a single component system is $24.2 \%$ compared to $2.79 \%$ for the RUD for the same system. There is no numerical dispersion error. While the values of the maximum relative errors appear to be large, they are in fact quite reasonable in view of the wide variations in magnitude of the size-composition distribution. For example, the maximum relative error for a single component system is $130 \%$. However the numerical solution at that maximum relative error is $5.15 \times 10^{-2}$ and the analytical solution is $2.24 \times 10^{-2}$. This numerical error is within an acceptable range of accuracy given the wide spread in the values in the solution. As another example, in Figure 2, the maximum relative error which occurs at the largest particle grid is $23 \%$. As mentioned earlier, the maximum relative error should be considered as the error of the solution at the point of the steepest gradient.

The overall performance of the TGFEM is also given in Table IV. Generally, the TGFEM performs moderately better than the RUD for both the median and maximum relative errors. During the simulation of single component dynamics by the TGFEM, negative distributions at the larger end were observed, but these negative numbers did not affect the overall performance of the scheme.

The changes of the total number and mass concentrations for pure condensation with linear rates are given in Table V. Ideally, the number ratio, ratio of the total number concentration at dimensionless time 1.0 to that at time zero, should be 1.0 , but due to the finite domain error (12), the analytical number ratios are not one. The changes of the numerical calculation are close to those of the analytical solution 
for the both methods. Though the TGFEM generally gives higher values than the analytical solutions, it is slightly better than the RUD. Another point is that during the simulation time, the total mass concentration increases by about a factor of two. Thus the system has undergone a significant change in its size-composition distribution.

A point to note is that the computing time increases substantially with increase of the number of components. This is due to the geometric increase of computational requirements with $n^{s}$.

Systems including evaporation with linear rates (cases 2 and 3) are also studied by the RUD. The results of pure evaporation and mixed condensation of a twocomponent system are summarized in Table VI, with the parameter values used and the resultant errors. Again a good agreement between numerical and exact solutions is obtained.

The effects of the choice of two parameters for the RUD, the scale factor of antidiffusive velocity and the number of iterations, have been tested by increasing the number of iterations up to two $(I O R D=3)$ and changing the scale factor from 0.9 to 1.10. Contrary to Smolarkiewicz's reports $(26,27)$, variation of these two parameters is found not to significantly enhance the performance of the numerical scheme consistently. By increasing IORD from 2 to 3 , the accuracy of single and three-component calculations slightly increases but the accuracy of two- and four-component calculations slightly decreases. The accuracy of the four-component calculation slightly increases with decrease of $S c$ from 1 to 0.9 while the accuracy of other component calculations slightly increased with increase of $S c$ from 1 to 1.1. Thus, the numerical results for the RUD with only one iteration and with the original antidiffusion rates are already greatly enhanced over those for the first-order upwind difference method and further iteration or/and ' change of the scale factor does not significantly improve the numerical results. One possible explanation for this insensitivity of the numerical results to the parameters after one iteration is that since the size-composition distribution changes by several orders of magnitude, the antidiffused amount at the 
first iteration step successfully subtracts the diffused amount, while Smolarkiewicz's examples exhibited small relative changes of the solution $(26,27)$.

Furthermore, the scale factor does not change the basic algorithm of the numerical scheme, Thus, the effects of the two parameters will not be studied further for pure condensation with one-third order rates.

Two-component aerosol dynamics with one-third order rates (diffusion-controlled condensation) is also studied (cases 4-6, pure growth, pure evaporation, and mixed growth/evaporation) to compare the two methods to a range of rate formulae. One characteristic of the one-third order rates is that the variation of the condensation rates with respect to the particle sizes is much larger than that of the linear rates. The analytical and numerical solutions by the RUD for pure condensation (case 4) are shown in Figures 3 and 4, respectively. Since the condensation rates of small particles are much larger than those of large particles, the size-composition distribution tends to sharpen, and the both the analytical and the numerical solutions clearly show this trend. The analytical and the numerical solutions for pure evaporation of a two-component aerosol system are shown in Figures 5 and 6 , respectively. The effect of evaporation is to shift the size-composition distribution to smaller sizes according to the characteristics of each component. The effect of mixed growth/evaporation is growth of the condensing component and shrinkage of the evaporating component, and the result is shown in Figures 7 and 8 for the analytical and the numerical solutions, respectively. The simulation results, computing times and errors, are given in Table VII. Note that the required computing time increases drastically since the time step is very small compared to that of case 1 due to the Courant number constraint which arises from the large variation of condensation rates. The numerical solutions are generally in fair agreement with the analytical solutions, though there are evident signs of numerical diffusion errors, spreading of the distribution and smearing peak values out.

The numerical diffusion errors develop noticeably because the size-composition distribution is very steep. The numerical simulation by the TGFEM produced nega- 
tive distribution values, and the number of grids having the negative values increased with time with the scheme becoming unstable about the dimensionless time 0.07 . Tsang and Rao (29) reported similar instability of the linear Galerkin method for single component aerosol condensation. Numerical dispersion errors are the cause of this instability. If the TGFEM with a nonlinear filter, for example Forester's filter, is used (5), there is still no guarantee of the non-negativity and therefore of stability.

\subsection{Pure Coagulation}

In this subsection, numerical results for pure coagulation (case 7) are studied. Since the coagulation process is the most time consuming part of the total solution, efforts are made to determine optimal computational parameters for this portion of the simulation.

In Figure 9, numerical and analytical solutions for a two-component coagulation system are shown. Good agreement between the numerical solutions and the analytical solutions was obtained. Contrary to the case of pure condensation, the sizecomposition distribution in Figure 9 shows only the shift of the peak to the largest particle size domain. This can be explained by the characteristics of coagulation. Table VIII also shows excellent performance of the numerical technique.

In Table IX, number and mass concentrations during coagulation are shown. A decrease of about $1 / 3$ of the particles occurs during the coagulation process. Generally the total mass concentrations are well conserved during the simulation except when the number of grids is small. Note that for a three-component system, the agreement between the analytical and the numerical ratio is better than those for other systems. This is due to the decrease of the computational domain for a three-component system, which is equivalent to increasing the number of grids.

Even with the use of the lower order accuracy schemes to minimize computing time, the computing time is still considerable (see Table VIII). By comparing the 
computing time required for calculating pure conderisation and pure coagulation (Tables IV and VIII), one can see that the computing time for pure coagulation far exceeds that for pure condensation and the differences increase with the number of components. The integration of the first term on the RHS of Eq. [7] is mainly responsible for these effects on the computing time. Integration of that term requires linear interpolation of a size-composition distribution at every Gaussian quadrature point for every grid point. Thus both the interpolation and the integration require substantial operations and consequently long computing time. Also both operations increase geometrically with the number of components For an $s$ component system with $n$ grids for each component, using a $q$ point product type Gaussian quadrature and the linear interpolation, $\left(n^{s} \times q^{s}+q^{s}\right)$ point calculations and $\left(2 \times s \times n^{s} \times q^{s}+s \times q^{s}\right)$ interpolations are required for each time step.

Several parameters are studied to find the optimal conditions for computing time and accuracy. Increasing the number of grids per component from 21 to 31 for twoand three-component cases gives a slight increase in accuracy but not enough to justify the subsequent increase of the computing time. The number of Gaussian quadrature points was also studied. A five-point Gaussian quadrature was found to represent a good compromise between accuracy and computing time; a three-point scheme gave results with poor accuracy while a seven-point scheme took too much computing time. Decreasing the time integration step by half produced a slight increase of accuracy for all cases but not sufficient to justify the computing time increase.

\subsection{Simultaneous Condensation and Coagulation}

We now address simultaneous condensation and coagulation processes (cases 8 and 9). Systems in which the changes of the size-composition distribution from both condensation and coagulation processes are of similar magnitude are studied here. Systems in which the magnitudes of these two processes are different are discussed in 
the next section.

Figure 10 presents the numerical and analytical results of a two-component condensation and coagulation system with linear condensation rates (case 8). Table $\mathrm{X}$ presents the computing time and the errors. The numerical solutions are in good agreement with the analytical solutions. Table XI gives the total number and mass concentration ratios. During simultaneous coagulation and condensation, neither total number nor total mass concentration is conserved as shown in Table XI. Comparing Figures 2, 9, and 10, we see that the size-composition distribution is primarily determined by condensation, but from Tables IV, VIII, and X, the computing time is dominated by the calculation of coagulation.

The effects of the choice of the two parameters used in the repeated upwind difference method were investigated since the size-composition distribution is primarily affected by condensation. As in the case of pure condensation, changing IORD did not affect the accuracy noticeably. Decreasing the values of $S c$ from 1 to 0.9 gave slight increased accuracy for all cases. Again, these two parameters have no apparent effect on the performance of the numerical scheme.

Figure 11 presents the numerical solution of a two-component simultaneous condensation and coagulation with one-third order rates (case 9). For this case, no analytical solution is available. The effect of coagulation is to move particles to larger sizes. Thus, the distribution has lower peak value than that of pure condensation.

\section{DISCUSSION}

The most significant attribute of the direct numerical solution of the multicomponent aerosol GDE is that for particles of the same size, the composition distribution is determined directly. The physical significance of this advantage can be illustrated by a simple example. 
Consider a two-component aerosol-gas system, with component A condensible and component $\mathrm{B}$ non condensible. There are $N$ particles of the same size but of two different compositions. The gas phase is supersaturated with $\mathrm{A}$ and only condensation occurs. After some time, there should be particles of two different sizes corresponding to growth of the two sets of initial particles. Say, for example, that there are initially equal numbers of monodisperse particles at dimensionless total mass of 0.930 with compositions of $88.1 \%$ and $11.9 \%$ of $\mathrm{A}$, respectively. The dimensionless condensation rate of $\mathrm{A}$ is 2 and that of $\mathrm{B}$ is 0 . At dimensionless time 1, the particles are of two different dimensionless total mass sizes, 6.161 and 1.638 , respectively. This example illustrates the importance of the composition distribution effect on the evolution of the size-composition distribution. Heintzenberg and Covert (15) also presented some examples for atmospheric aerosol particles that demonstrate the importance of the composition distribution in one size range.

An important difference between the method developed here and the sectional method is that the latter calculates moments within the sections, usually the mass moment, while the direct method calculates the size-composition distribution itself. Therefore, the difference between the direct method and the sectional method is also reflected in the nature of the numerical results, in addition to the accuracy or the computing time, since the methods compute different quantities.

Integration of the size-composition distribution results in the mass concentration as in the sectional method. But during the integration, information about the composition distribution is lost. Thus, even if the two methods present comparable results with integration, it does not mean that the additional cost of computing time cannot be justified since one may want to know not only the mass concentration but also the composition distribution. Including sources and removal processes in the proposed numerical scheme is straightforward. This is done by including these processes with the coagulation calculation.

The effect of the numerical boundary condition has been studied by comparing numerical solutions obtained with the analytical boundary condition to those with the 
numerical boundary condition for pure condensation with linear rates and the RUD. As shown in Table XII, the two solutions exhibit almost no difference. Therefore, the use of the chosen numerical boundary conditions can be justified for the simulation of cases 4-6 and 9 (cases with one-third order condensation rates).

In Table XIII, a comparison of the computing times of direct solutions and sectional solutions is given. The computing times of the direct solution are less than those of sectional solutions for the single component system. But the computing times of the direct solution increase with the number of components and are about a factor of 10 and a factor of 1000 longer than those of sectional solutions for two- and threecomponent systems, respectively. For many real systems of two-component aerosols, considering the quality of the solution obtained, 20 times longer computing time is not considered as a major disadvantage. The direct solution of the three-component GDE is the order of 100 times longer than the sectional method. The ratio of the computing time of the direct solution method to that of the moving grid sectional method for pure condensation is 473 . Note that in the moving grid sectional method the computing time is linearly related to the number of grids.

The ratio of computing time of the direct method to that of the fixed grid sectional method for pure coagulation is much larger than that for the cases of condensation processes, namely for the example considered here, 1168. In both the sectional method and direct method, simulation of the coagulation process is the most time consuming part as already shown in Table XIII. Double integrals must be evaluated in the sectional method regardless of the number of components while triple integrals must be evaluated in the direct method for the three-component GDE ( $n$ integrals for an $n$ component system). Note that the computing time increases about five times for a doubling of the number of grids in the sectional method. With ten sections, one can simplify the double integrals by imposing the so-called geometric constraint, $m_{i, j+1} \leq 2 m_{i, j}(14)$. But with 21 sections, the geometric constraint is no longer applicable.

For the cases of simultaneous condensation and coagulation, the computing times 
of both methods are determined, in principle, by both the coagulation and the condensation processes as shown in Table $\mathrm{X}$, but the ratio of computing time for the coagulation portion over that of the condensation portion is about 45 . Thus the use of the linear interpolation method for the coagulation simulation is basically a compromise between accuracy and computing time.

With respect to the required computer memory for an $s$-component GDE with $n$ grids per each component, $n^{s}$ functional values represent the size distribution, while in the sectional method, only $s \times n$ functional values represent the moment distribution.

One interesting problem is whether the integrated results of the direct solution and those of the sectional solution are comparable. For a single component case, it is expected that the two methods produce comparable results and that is the case. For a two-component case, the pure condensation result is shown in Figure 12; both results are accurate and comparable. This similarity is due to the system itself. Since the chosen system has similar condensation rates for each component and similar initial component mass, the mean mass fractions represent well the real situations and there is no severe difference between results as the example given before in which the mean mass fractions misrepresented the real situation. Also, still one can see the difference of mass concentrations just after the peak.

For the pure coagulation case, the two results are almost equivalent. Gelbard (private communication) suggested that since the coagulation process is composition independent (with composition independent coagulation coefficients), the two methods may produce similar results.

The computational demands of the direct solution may not always be so severe. In many cases, the time variation of only one species is of special interest. One notable example is the dynamics of water in a nuclear reactor containment system where the condensation rate of water is much larger than that of other species. In such cases one can specify a large number of grids for the component of interest while maintaining a rather smaller number of grids for other components. Table XIV illustrates one example in such a case, where in a two-component system, one 
component condenses nine times faster than the other while coagulation proceeds simultaneously. By reducing the number of grids of the less condensible component from 21 to 7 , the computing time decreased from 546.2 to 171.0 seconds with only a slight decrease in accuracy.

In some cases, $\Lambda_{i}$, the ratio of the characteristic time for coagulation to that for condensation of the $i$-th component may be very large. In such a case, one can simulate several condensation steps over one coagulation step. One such example is presented in Table XV. Two condensation steps were calculated per one coagulation step and the results show the computing time is reduced to about half of the original calculation with modest loss of accuracy. Also this example illustrates well the difficulty in applying the time splitting method to simultaneous processes. Since the characteristic time of condensation is smaller than that of coagulation by a factor of about 5.5, using the same time step as when the characteristic times for the two processes are equal gives erroneous results (see the two-component results in Table VI and Table IX). Thus, the time step should be reduced in accord with the smallest characteristic time.

\section{CONCLUSIONS}

A numerical scheme is developed to simulate the evolution of the number sizecomposition distribution of a multicomponent aerosol system with special attention to simultaneous condensation and coagulation. The scheme does not adopt any assumptions on the size-composition distribution. Smolarkiewicz's repeated upwind difference method and the Taylor-Galerkin method are used to simulate the condensation process, a product type Gaussian quadrature with linear interpolation method is used to simulate the coagulation process, and the operator splitting method is used to combine the two methods. 


\section{ACKNOWLEDGMENT}

This work was supported by National Science Foundation grant ATM9003186.

Also we thank Dr. F. Gelbard for helpful discussions. 


\section{REFERENCES}

1. Ahlberg, J.H. Nilsen, E.N. and Walsh, J.L., "The Theory of Spline and Their Applications". Academic Press, New York, 1967.

2. Artaxo, P, Maenhaut, W. Storms, H. and Van Grieken, R., J. Geo. Res. 95, 16971 (1990).

3. Bassett, M., Gelbard, F. and Seinfeld, J.H., Atmos. Environ. 15, 2395 (1981).

4. Boris, J.P. and Book, D.L., J. Comp. Phys. 1138 (1973).

5. Chock, D.P., Atmos. Environ. 24A, 1 (1990).

6. Davis, P.J. and Rabinowitz, R., "Methods of Numerical Integration". Academic Press, New York, 1975.

7. Donea, J., Int'l J. Numer. Meth. in Eng. 20, 101 (1984).

8. Flagan, R.C., in "Aerosols: Science, Industry, Health and Environment", Masuda, S. and Takahashi, K. Ed., pp 50-54. Pergamon Press, Oxford, 1990.

9. Gelbard, F., "Proceedings of American Association for Aerosol Research", Seattle, 1987.

10. Gelbard, F., Aerosol Sci. Technol. 12, 399 (1990).

11. Gelbard, F. and Seinfeld, J.H., J. Colloid Interface Sci. 63, 472 (1978).

12. Gelbard, F. and Seinfeld, J.H., J. Comp. Phys. 28, 357 (1978).

13. Gelbard, F. and Seinfeld, J.H., J. Colloid Interface Sci. 68, 363 (1979).

14. Gelbard, F. and Seinfeld, J.H., J. Colloid Interface Sci. 78, 485 (1980).

15. Heintzenberg, J. and Covert, D.S., J. Atmos. Chem. 10, 383 (1990).

16. Kim, Y.P. and Seinfeld, J.H., J. Colloid Interface Sci. 135, 185 (1990).

17. Lapidus, L. and Pinder, G.F., "Numerical Solution of Partial Differential Equation in Science and Engineering”. John Wiley \& Sons, New York, 1982.

18. Middleton, P. and Brock, J., J. Colloid Interface Sci. 54, 249 (1976).

19. Nazaroff, W.W. and Cass, G.R., J. Environ. Sci. Technol. 23, 157 (1989).

20. Oran, E.S. and Boris, J.P., "Numerical Simulation of Reactive Flow". Elsevier, New York, 1987.

21. Pilinis, C. and Seinfeld, J.H., Atmos. Environ. 22, 1985 (1988). 
22. Pilinis, C., Seinfeld, J.H., and Seigneur, C., Atmos. Environ. 21, 943 (1987).

23. Russell, A.G. and Cass, G.R., Atmos. Environ. 18, 1815 (1984).

24. Seinfeld, J.H., "Atmospheric Chemistry and Physics of Air Pollution". John Wiley \& Sons, New York, 1986.

25. Simons, S., Annual Nuclear Energy 9, 473 (1982).

26. Smolarkiewicz, P.K., Mon. Wea. Rev. 111, 479 (1983).

27. Smolarkiewicz, P.K., J. Comp. Phys. 54, 325 (1984).

28. Stroud, A.H., "Approximate Calculation of Multiple Integrals". Prentice-Hall, Englewood Cliffs, 1971.

29. Tsang, T.H. and Brock, J.R., Aerosol Sci. Technol. 2, 311 (1983).

30. Tsang, T.H. and Rao, A., Aerosol Sci. Technol. 9, 271 (1988).

31. Tsang, T.H. and Rao, A., Int'l J. Numer. Meth. in Fluids 10, 753 (1990).

32. Varoḡlu, E and Liam Finn, W.D., J. Comp. Phys. 34, 371 (1980).

33. Villadsen, J. and Michelsen, M.L., "Solution of Differential Equation Models by Polynomial Approximation”. Prentice-Hall. Englewood Cliffs, 1978.

34. Warren, D.R., "Ph. D. Thesis". California Institute of Technology, Pasadena, 1986.

35. Warren, D.R. and Seinfeld, J.H., Aerosol Sci. Technol. 4, 31 (1985).

36. Rood, R.B., Rev. of Geophys. 25, 71 (1987). 
Table I. Cases Studied in Multicomponent Aerosol Dynamics

\begin{tabular}{llll} 
Case & Process (es) & Rate form & No. Comp. \\
\hline 1 & Growth & $I_{i}=\alpha_{i} m_{i}$ & $1-4$ \\
2 & Evaporation & $I_{i}=\alpha_{i} m_{i}$ & 2 \\
3 & Grow./Evap. & $I_{i}=\alpha_{i} m_{i}$ & 2 \\
4 & Growth & $I_{i}=\alpha_{i}\left(\sum_{j=1}^{s} m_{j}\right)^{1 / 3}$ & 2 \\
5 & Evaporation & $I_{i}=\alpha_{i}\left(\sum_{j=1}^{s} m_{j}\right)^{1 / 3}$ & 2 \\
6 & Grow./Evap. & $I_{i}=\alpha_{i}\left(\sum_{j=1}^{s} m_{j}\right)^{1 / 3}$ & 2 \\
7 & Coagulation & $\beta=\beta_{o}$ & $1-4$ \\
8 & Condensation & $I_{i}=\alpha_{i} m_{i}$ & $1-4$ \\
& and & \\
9 & Coagulation & $\beta=\beta_{o}$ \\
& Condensation & $I_{i}=\alpha_{i}\left(\sum_{j=1}^{s} m_{j}\right)^{1 / 3}$ & 2 \\
& and & \\
\hline
\end{tabular}

${ }^{1}$ No analytical solution is available. 
Table II. Parameters for the Simulations

\begin{tabular}{llcc} 
Case & $\begin{array}{l}\text { Number of } \\
\text { Components }\end{array}$ & $m_{i o} / M_{o}$ & $\Lambda_{i}^{1}$ \\
\hline 1,8 & 1 & 1.0 & 1.0 \\
$1,4,8,9$ & 2 & $0.5,0.5$ & $0.9,1.1$ \\
1,8 & 3 & $0.33,0.33,0.34$ & $0.9,1.0,1.1$ \\
1,8 & 4 & $0.25,0.25,0.25,0.25$ & $0.9,0.8,1.3,1.0$ \\
\hline
\end{tabular}

${ }^{1}$ Case 1 (pure condensation, linear rates): $\Lambda_{i}=\frac{\alpha_{i}}{\alpha_{0}}$.

Case 4 (pure condensation, one-third order rates): $\Lambda_{i}=\frac{\alpha_{i}}{\alpha_{0}}$.

Case 7 (linear condensation rates, coagulation): $\Lambda_{i}=\frac{\alpha_{i}}{\beta_{o} N_{o}}$. 
Table III. Summary of Calculational Parameters Used ${ }^{1}$

\begin{tabular}{llll} 
Case & $\begin{array}{l}\text { Number of } \\
\text { Components }\end{array}$ & $\begin{array}{l}\text { Number of Grids per } \\
\text { Each Components }\end{array}$ & $\begin{array}{l}\text { Computational } \\
\text { domain }\left(\frac{m_{i}}{M_{o}}\right)\end{array}$ \\
\hline $1,7,8$ & 1 & 21 & $1 \times 10^{-4}, 2 \times 10^{1}$ \\
$1-9$ & 2 & 21 & $5 \times 10^{-4}, 5 \times 10^{0}$ \\
1 & $3^{2}$ & 21 & $5 \times 10^{-4}, 3 \times 10^{0}$ \\
7,8 & $3^{3}$ & 21 & $5 \times 10^{-3}, 3 \times 10^{0}$ \\
1 & $4^{2}$ & 13 & $5 \times 10^{-4}, 2 \times 10^{0}$ \\
7,8 & $4^{3}$ & 7 & $5 \times 10^{-3}, 2 \times 10^{0}$ \\
\hline
\end{tabular}

${ }^{1}$ Dimensionless time step $\Delta t$ is 0.01 for all cases except cases 4,5 , and 8 for which $\Delta t$ is $2 \times 10^{-5}$.

${ }^{2}$ For the cases of pure condensation/evaporation.

${ }^{3}$ For the cases including coagulation. 
Table IV. Summary of Condensation Simulations (Case 1)

\begin{tabular}{llll}
$\begin{array}{l}\text { Number of } \\
\text { Components }\end{array}$ & $\begin{array}{l}\text { Computing } \\
\text { Time }(\mathrm{s})^{1}\end{array}$ & $\begin{array}{l}\text { Median Relative } \\
\text { Error }(\%)^{2}\end{array}$ & $\begin{array}{l}\text { Maximum Relative } \\
\text { Error }(\%)^{3}\end{array}$ \\
\hline
\end{tabular}

The Repeated Upwind Difference Method

$\begin{array}{llll}1 & 0.6 & 2.79 & 130 \\ 2 & 22.9 & 5.36 & 23.0 \\ 3 & 1341 & 4.55 & 36.9 \\ 4 & 9462 & 15.1 & 28.7\end{array}$

The Taylor-Galerkin Method

\begin{tabular}{llll}
1 & 0.8 & 0.39 & 50.2 \\
2 & 36.6 & 3.14 & 12.2 \\
3 & 1827 & 5.55 & 11.4 \\
4 & 10475 & 12.1 & 22.6 \\
\hline
\end{tabular}

${ }^{1}$ SUN SPARC station.

${ }^{2}$ Median Relative Error $(\%)=\operatorname{median}\left[\frac{\operatorname{abs}\left(\left.n(m, t)\right|_{\text {analytical }}-\left.n(m, t)\right|_{\text {numerical }}\right)}{\left.n(m, t)\right|_{\text {analytical }}} \times 100\right]$

${ }^{3}$ Maximum Relative Error $(\%)=\operatorname{Max}\left[\frac{\operatorname{abs}\left(\left.n(m, t)\right|_{\text {analytical }}-\left.n(m, t)\right|_{\text {numerical }}\right)}{\left.n(m, t)\right|_{\text {analytical }}} \times 100\right]$ 
Table V. Total Number ${ }^{1}$ and Mass ${ }^{2}$ Concentration Ratios for Pure Condensation (Case 1)

$\begin{array}{llll}\text { Number of } & \text { Analytical } & \text { Numerical } & \text { Numerical } \\ \text { Components } & \text { Ratio } & \text { Ratio } & \text { Ratio } \\ & & \left(\text { RUD }^{3}\right) & \left(\text { TGFEM }^{4}\right)\end{array}$

\begin{tabular}{lllll}
\hline & & & & \\
Number & 1 & 0.999 & 0.982 & 1.001 \\
& 2 & 0.949 & 0.903 & 0.953 \\
& 3 & 0.894 & 0.835 & 0.831 \\
& 4 & 0.790 & 0.682 & 0.809 \\
\hline \multirow{4}{*}{ Mass } & & & & \\
& 1 & 2.70 & 2.72 & 2.75 \\
& 3 & 2.33 & 2.11 & 2.34 \\
& 4 & 2.64 & 1.89 & 2.14 \\
& 4 & 1.79 & 1.42 & 1.80 \\
\hline
\end{tabular}

${ }^{1}$ Ratio of total number concentration at $\tau=1$ to that at $\tau=0$;

$$
\int_{m_{1}^{\alpha}}^{m_{1}^{\beta}} \cdots \int_{m_{s}^{\alpha}}^{m_{s}^{\beta}} n(m, \tau=1) d m / \int_{m_{1}^{\alpha}}^{m_{1}^{\beta}} \cdots \int_{m_{s}^{\alpha}}^{m_{s}^{\beta}} n(m, \tau=0) d m
$$

${ }^{2}$ Ratio of total mass concentration at $\tau=1$ to that at $\tau=0$;

$$
\sum_{j=1}^{s} \int_{m_{1}^{\alpha}}^{m_{1}^{\beta}} \cdots \int_{m_{s}^{\alpha}}^{m_{s}^{\beta}} m_{j} n(m, \tau=1) d m / \sum_{j=1}^{s} \int_{m_{1}^{\alpha}}^{m_{1}^{\beta}} \cdots \int_{m_{s}^{\alpha}}^{m_{s}^{\beta}} m_{j} n(m, \tau=0) d m
$$

${ }^{3}$ RUD: The repeated upwind difference method.

${ }^{4}$ TGFEM: The Taylor-Galerkin method. 
Table VI. Summary of Growth/Evaporation Simulations

(Cases 2 and 3, Two-Component)

\begin{tabular}{lllll} 
Process & $\begin{array}{l}\text { Dimensionless } \\
\text { Rates, } \Lambda_{i}\end{array}$ & $\begin{array}{l}\text { Computing } \\
\text { Time }(\mathrm{s})^{\mathbf{1}}\end{array}$ & $\begin{array}{l}\text { Median } \\
\text { Relative } \\
\text { Error(\%) }\end{array}$ & $\begin{array}{l}\text { Maximum } \\
\text { Relative } \\
\text { Error }(\%)^{3}\end{array}$ \\
\hline $\begin{array}{l}\text { Pure Evapora- } \\
\text { tion (case 2) }\end{array}$ & $-0.1,-0.1$ & 27.2 & 1.37 & 92.3 \\
$\begin{array}{l}\text { Mixed } \\
\text { (case 3) }\end{array}$ & $-0.1,1.1$ & 27.5 & 2.26 & 43.9 \\
\hline
\end{tabular}

\section{${ }^{1}$ SUN $386 i$.}

${ }^{2}$ Median Relative Error $(\%)=\operatorname{median}\left[\frac{\mathrm{abs}\left(\left.n(m, t)\right|_{\text {lnalytical }}-\left.n(m, t)\right|_{\text {numerical }}\right)}{\left.n(m, t)\right|_{\text {analytical }}} \times 100\right]$

${ }^{3}$ Maximum Relative Error $(\%)=\operatorname{Max}\left[\frac{\text { abs }\left(\left.n(m, t)\right|_{\text {analytical }}-\left.n(m, t)\right|_{\text {numerical }}\right)}{\left.n(m, t)\right|_{\text {analytical }}} \times 100\right]$ 
Table VII. Summary of Condensation Simulations (Cases 4-6, Two-Component)

\begin{tabular}{lllll} 
Process & $\begin{array}{l}\text { Dimensionless } \\
\text { Rates, } \Lambda_{i}\end{array}$ & $\begin{array}{l}\text { Computing } \\
\text { Time }(\mathrm{s})^{1}\end{array}$ & $\begin{array}{l}\text { Median } \\
\text { Relative } \\
\text { Error(\%) }\end{array}$ & $\begin{array}{l}\text { Maximum } \\
\text { Relative } \\
\text { Error (\%) }\end{array}$ \\
\hline $\begin{array}{l}\text { Pure } \\
\begin{array}{l}\text { Condensa- } \\
\text { tion (case 4) }\end{array}\end{array}$ & $0.9,1.1$ & 8450 & 92 & 100 \\
$\begin{array}{l}\text { Pure Evapora- } \\
\text { tion (case 5) }\end{array}$ & $-0.9,-1.1$ & 9640 & 71 & 147 \\
$\begin{array}{l}\text { Mixed } \\
\text { (case 6) }\end{array}$ & $-0.9,1.1$ & 9100 & 93 & 393 \\
\hline
\end{tabular}

${ }^{1}$ SUN $386 i$ i.

${ }^{2}$ Median Relative Error $(\%)=\operatorname{median}\left[\frac{\operatorname{abs}\left(\left.n(m, t)\right|_{\text {analytical }}-\left.n(m, t)\right|_{\text {numerical }}\right)}{\left.n(m, t)\right|_{\text {analytical }}} \times 100\right]$

${ }^{3}$ Maximum Relative Error $(\%)=\operatorname{Max}\left[\frac{\text { abs }\left(\left.n(m, t)\right|_{\text {analytical }}-\left.n(m, t)\right|_{\text {numerical }}\right)}{\left.n(m, t)\right|_{\text {analytical }}} \times 100\right]$ 
Table VIII. Summary of Coagulation Simulations (Case 7)

\begin{tabular}{llll}
$\begin{array}{l}\text { Number of } \\
\text { Components }\end{array}$ & $\begin{array}{l}\text { Computing } \\
\text { Time }(\mathrm{s})^{1}\end{array}$ & $\begin{array}{l}\text { Median Relative } \\
\text { Error }(\%)^{2}\end{array}$ & $\begin{array}{l}\text { Maximum Relative } \\
\text { Error }(\%)^{3}\end{array}$ \\
\hline 1 & 4.4 & 8.17 & 95.9 \\
2 & 408 & 4.81 & 16.1 \\
3 & 40541 & 1.95 & 25.2 \\
4 & 59908 & 8.19 & 67.4 \\
\hline
\end{tabular}

${ }^{1}$ SUN 386 i.

${ }^{2}$ Median Relative Error $(\%)=\operatorname{median}\left[\frac{\mathrm{abs}\left(\left.n(m, t)\right|_{\text {lanalytical }}-\left.n(m, t)\right|_{\text {numerical }}\right)}{\left.n(m, t)\right|_{\text {analytical }}} \times 100\right]$

${ }^{3}$ Maximum Relative Error $(\%)=\operatorname{Max}\left[\frac{\text { abs }\left(\left.n(m, t)\right|_{\text {lnalytical }}-\left.n(m, t)\right|_{\text {numerical }}\right)}{\left.n(m, t)\right|_{\text {analytical }}} \times 100\right]$ 
Table IX. Total Number ${ }^{1}$ and Mass ${ }^{2}$ Concentration Ratios for Pure Coagulation (Case 7)

\begin{tabular}{llll} 
& $\begin{array}{l}\text { Number of } \\
\text { Components }\end{array}$ & $\begin{array}{l}\text { Analytical } \\
\text { Ratio }\end{array}$ & $\begin{array}{l}\text { Numerical } \\
\text { Ratio }\end{array}$ \\
\hline \multirow{3}{*}{ Number } & 1 & & \\
& 2 & 0.667 & 0.595 \\
& 3 & 0.666 & 0.622 \\
& 4 & 0.672 & 0.676 \\
& & 0.674 & 0.527 \\
& & & \\
Mass & & & 0.972 \\
& 2 & 1.00 & 0.910 \\
& 3 & 0.988 & 0.988 \\
& 4 & 0.989 & 0.810 \\
\hline
\end{tabular}

${ }^{1}$ Ratio of total number concentration at $\tau=1$ to that at $\tau=0$;

$$
\int_{m_{1}^{\alpha}}^{m_{1}^{\beta}} \ldots \int_{m_{s}^{\alpha}}^{m_{s}^{\beta}} n(m, \tau=1) d m / \int_{m_{1}^{\alpha}}^{m_{1}^{\beta}} \ldots \int_{m_{s}^{\alpha}}^{m_{s}^{\beta}} n(m, \tau=0) d m
$$

${ }^{2}$ Ratio of total mass concentration at $\tau=1$ to that at $\tau=0$;

$$
\sum_{j=1}^{s} \int_{m_{1}^{\alpha}}^{m_{1}^{\beta}} \ldots \int_{m_{s}^{\alpha}}^{m_{s}^{\beta}} m_{j} n(m, \tau=1) d m / \sum_{j=1}^{s} \int_{m_{1}^{\alpha}}^{m_{1}^{\beta}} \ldots \int_{m_{s}^{\alpha}}^{m_{s}^{\beta}} m_{j} n(m, \tau=0) d m
$$


Table X. Summary of Simultaneous Condensation and Coagulation Simulations (Case 8)

\begin{tabular}{llll}
$\begin{array}{l}\text { Number of } \\
\text { Components }\end{array}$ & $\begin{array}{l}\text { Computing } \\
\text { Time }(\mathrm{s})^{1}\end{array}$ & $\begin{array}{l}\text { Median Relative } \\
\text { Error }(\%)^{2}\end{array}$ & $\begin{array}{l}\text { Maximum Relative } \\
\text { Error }(\%)^{3}\end{array}$ \\
\hline 1 & 4.9 & 2.62 & 27.8 \\
2 & 431 & 3.34 & 34.6 \\
3 & 43035 & 1.54 & 34.2 \\
4 & 60217 & 24.0 & 66.6 \\
\hline
\end{tabular}

1 SUN 386i.

${ }^{2}$ Median Relative Error $(\%)=\operatorname{median}\left[\frac{\operatorname{abs}\left(\left.n(m, t)\right|_{\text {lnalytical }}-\left.n(m, t)\right|_{\text {numerical }}\right)}{\left.n(m, t)\right|_{\text {analytyical }}} \times 100\right]$

${ }^{3}$ Maximum Relative Error $(\%)=\operatorname{Max}\left[\frac{\text { abs }\left(\left.n(m, t)\right|_{\text {analytical }}-\left.n(m, t)\right|_{\text {numerical }}\right)}{\left.n(m, t)\right|_{\text {analytycal }}} \times 100\right]$ 
Table XI. Total Number ${ }^{1}$ and Mass $^{2}$ Concentration Ratios for Simultaneous Condensation and Coagulation (Case 8)

\begin{tabular}{|c|c|c|c|}
\hline & $\begin{array}{l}\text { Number of } \\
\text { Components }\end{array}$ & $\begin{array}{l}\text { Analytical } \\
\text { Ratio }\end{array}$ & $\begin{array}{l}\text { Numerical } \\
\text { Ratio }\end{array}$ \\
\hline Number & $\begin{array}{l}1 \\
2 \\
3 \\
4\end{array}$ & $\begin{array}{l}0.662 \\
0.566 \\
0.514 \\
0.432\end{array}$ & $\begin{array}{l}0.597 \\
0.542 \\
0.521 \\
0.357\end{array}$ \\
\hline Mass & $\begin{array}{l}1 \\
2 \\
3 \\
4\end{array}$ & $\begin{array}{l}2.60 \\
1.71 \\
1.41 \\
1.07\end{array}$ & $\begin{array}{l}2.30 \\
1.55 \\
1.40 \\
0.829\end{array}$ \\
\hline
\end{tabular}

${ }^{1}$ Ratio of total number concentration at $\tau=1$ to that at $\tau=0$;

$$
\int_{m_{1}^{\alpha}}^{m_{1}^{\beta}} \cdots \int_{m_{s}^{\alpha}}^{m_{s}^{\beta}} n(m, \tau=1) d m / \int_{m_{1}^{\alpha}}^{m_{1}^{\beta}} \cdots \int_{m_{s}^{\alpha}}^{m_{s}^{\beta}} n(m, \tau=0) d m
$$

${ }^{2}$ Ratio of total mass concentration at $\tau=1$ to that at $\tau=0$;

$$
\sum_{j=1}^{s} \int_{m_{1}^{\alpha}}^{m_{1}^{\beta}} \cdots \int_{m_{s}^{\alpha}}^{m_{s}^{\beta}} m_{j} n(m, \tau=1) d m / \sum_{j=1}^{s} \int_{m_{1}^{\alpha}}^{m_{1}^{\beta}} \cdots \int_{m_{s}^{\alpha}}^{m_{s}^{\beta}} m_{j} n(m, \tau=0) d m
$$


Table XII. The Effect of Numerical Boundary Condition on the Numerical Solution (Two-Component System) ${ }^{1}$

\begin{tabular}{lllll} 
Case & $\begin{array}{l}\text { Number } \\
\text { Ratio }^{2}\end{array}$ & $\begin{array}{l}\text { Mass } \\
\text { Ratio }^{3}\end{array}$ & $\begin{array}{l}\text { Median Relative } \\
\text { Error(\%) }\end{array}$ & $\begin{array}{l}\text { Maximum Relative } \\
\text { Error (\%) }\end{array}$ \\
\hline 1 & 0.901 & 2.19 & 3.24 & 32.4 \\
& $(0.901)$ & $(2.11)$ & $(8.00)$ & $(23.0)$ \\
7 & 0.621 & 0.909 & 4.79 & 16.1 \\
& $(0.622)$ & $(0.910)$ & $(4.81)$ & $(16.1)$ \\
8 & 0.541 & 1.55 & 3.22 & 34.6 \\
& $(0.542)$ & $(1.55)$ & $(3.34)$ & $(34.6)$ \\
\hline
\end{tabular}

${ }^{1}$ Numbers in parenthesis are values using the analytical boundary condition.

${ }^{2}$ Ratio of total number concentration at $\tau$ to that at $\tau=0$;

$$
\int_{m_{1}^{\alpha}}^{m_{1}^{\beta}} \cdots \int_{m_{s}^{\alpha}}^{m_{s}^{\beta}} n(m, \tau) d m / \int_{m_{1}^{\alpha}}^{m_{1}^{\beta}} \cdots \int_{m_{s}^{\alpha}}^{m_{s}^{\beta}} n(m, \tau=0) d m
$$

${ }^{3}$ Ratio of total mass concentration at $\tau$ to that at $\tau=0$;

$$
\sum_{j=1}^{s} \int_{m_{1}^{\alpha}}^{m_{1}^{\beta}} \cdots \int_{m_{s}^{\alpha}}^{m_{s}^{\beta}} m_{j} n(m, \tau) d m / \sum_{j=1}^{s} \int_{m_{1}^{\alpha}}^{m_{1}^{\beta}} \ldots \int_{m_{s}^{\alpha}}^{m_{s}^{\beta}} m_{j} n(m, \tau=0) d m
$$

${ }^{4}$ Median Relative Error $(\%)=\operatorname{median}\left[\frac{\operatorname{abs}\left(\left.n(m, t)\right|_{\text {analytical }}-\left.n(m, t)\right|_{\text {numerical }}\right)}{\left.n(m, t)\right|_{\text {analytical }}} \times 100\right]$

${ }^{5}$ Maximum Relative Error $(\%)=\operatorname{Max}\left[\frac{\operatorname{abs}\left(\left.n(m, t)\right|_{\text {analytical }}-\left.n(m, t)\right|_{\text {numerical }}\right)}{\left.n(m, t)\right|_{\text {analytical }}} \times 100\right]$ 
Table XIII. Comparison of Computing Times

between the Direct Solution and the Sectional Solution

\begin{tabular}{|c|c|c|c|c|c|c|c|c|}
\hline$\frac{\text { Process }}{\text { Number of }}$ & \multirow[t]{2}{*}{$\begin{array}{l}\text { Numerical } \\
\text { Method }\end{array}$} & \multirow{2}{*}{$\begin{array}{l}\text { Number } \\
\text { of } \\
\text { Grids }^{1}\end{array}$} & \multicolumn{3}{|c|}{$\begin{array}{c}\text { Computing } \\
\text { Time }(s)^{2}\end{array}$} & \multicolumn{3}{|c|}{$\begin{array}{l}\text { Computing } \\
\text { Time Ratio }\end{array}$} \\
\hline Components & & & 1 & 2 & 3 & 1 & 2 & 3 \\
\hline Condensation/ & $\mathrm{MSM}^{3}$ & 10 & 0.3 & 1.0 & 0.9 & 0.50 & 0.83 & 0.47 \\
\hline evaporation & MSM & 21 & 0.6 & 1.2 & 1.9 & 1 & 1 & 1 \\
\hline only (case 1 ) & $\mathrm{DM}^{4}$ & 21 & 0.4 & 24.2 & 899 & 0.67 & 20.2 & 473 \\
\hline Coagulation & $\mathrm{FSM}^{5}$ & 10 & 2.5 & 4.8 & 5.8 & 0.16 & 0.14 & 0.17 \\
\hline only & FSM & 21 & 15.3 & 33.3 & 34.7 & 1 & 1 & 1 \\
\hline (case 7$)$ & DM & 21 & 4.4 & 408 & 40541 & 0.29 & 12.3 & 1168 \\
\hline $\begin{array}{l}\text { Simultaneous } \\
\text { condensation } \\
\text { and } \\
\text { coagulation } \\
\text { (case } 8 \text { ) }\end{array}$ & $\begin{array}{l}\text { FSM } \\
\text { FSM } \\
\text { DM }\end{array}$ & $\begin{array}{l}10 \\
21 \\
21\end{array}$ & $\begin{array}{r}2.4 \\
15.1 \\
4.9\end{array}$ & $\begin{array}{r}4.9 \\
32.7 \\
431\end{array}$ & $\begin{array}{r}5.4 \\
33.6 \\
43035\end{array}$ & $\begin{array}{r}0.16 \\
1 \\
0.31\end{array}$ & $\begin{array}{r}0.16 \\
1 \\
13.2\end{array}$ & $\begin{array}{r}0.16 \\
1 \\
1281\end{array}$ \\
\hline
\end{tabular}

${ }^{1}$ Direct method: number of grids per each component.

Sectional method: total number of sections.

2 SUN 386i.

${ }^{3}$ Moving grid sectional method.

${ }^{4}$ Direct Solution.

${ }^{5}$ Fixed grid sectional method. 
Table XIV. A Case of Reducing Computing Time:

Two-Component Condensation with Different Condensation Rates ${ }^{1}$ (Case 1)

\begin{tabular}{llllll} 
Compo- & $\Lambda_{i}$ & Number & Computing & Median Relative & Maximum Relative \\
nents & & of Grids & Time $(\mathrm{s})^{2}$ & Error $(\%)^{3}$ & Error $(\%)^{4}$ \\
\hline & & & & & \\
1 & 1.8 & 21 & 171.0 & 9.42 & 32.1 \\
2 & 0.2 & 7 & & & \\
\hline
\end{tabular}

1 With 21 grids per component,

Computing time : 546.2 seconds

Median relative error : $5.79 \%$

Maximum relative error : $31.3 \%$

2 SUN 386i.

${ }^{3}$ Median Relative Error $(\%)=\operatorname{median}\left[\frac{\operatorname{abs}\left(\left.n(m, t)\right|_{\text {analytical }}-\left.n(m, t)\right|_{\text {numerical }}\right)}{\left.n(m, t)\right|_{\text {analytical }}} \times 100\right]$

${ }^{4}$ Maximum Relative Error $(\%)=\operatorname{Max}\left[\frac{\operatorname{abs}\left(\left.n(m, t)\right|_{\text {analytical }}-\left.n(m, t)\right|_{\text {numerical }}\right)}{\left.n(m, t)\right|_{\text {analytical }}} \times 100\right]$ 
Table XV. A Case of Reducing Computing Time:

Two-Component Condensation and Coagulation with Fast Condensation ${ }^{1}$ (Case 8)

Compo- $\Lambda_{i}$ Number Computing Median Relative Maximum Relative nents $\quad$ of Grids $\quad$ Time $(\mathrm{s})^{2} \quad$ Error $(\%)^{3} \quad$ Error $(\%)^{4}$

$\begin{array}{llllll}1 & 5.0 & 21 & 287.8 & 38.8 & 363\end{array}$

$2 \quad 6.0 \quad 21$

${ }^{1}$ Two condensation steps calculation per coagulation step calculation.

With usual simulation of one condensation step calculation per one coagulation step calculation,

Computing time : 554 seconds

Median relative error : $39.2 \%$

Maximum relative error : $283 \%$

${ }^{2}$ SUN $386 i$.

${ }^{3}$ Median Relative Error $(\%)=\operatorname{median}\left[\frac{\text { abs }\left(\left.n(m, t)\right|_{\text {lnalytical }}-\left.n(m, t)\right|_{\text {numerical }}\right)}{\left.n(m, t)\right|_{\text {analytical }}} \times 100\right]$

${ }^{4}$ Maximum Relative Error $(\%)=\operatorname{Max}\left[\frac{\operatorname{abs}\left(\left.n(m, t)\right|_{\text {analytical }}-\left.n(m, t)\right|_{\text {numerical }}\right)}{\left.n(m, t)\right|_{\text {analytical }}} \times 100\right]$ 


\section{FIGURE CAPTIONS}

Figure 1. Initial exponential dimensionless number distribution of a two-component aerosol system.

Figure 2. Analytical solution (solid line) and numerical solution (broken line) of dimensionless number distribution of a two-component aerosol system with pure growth following linear rates at dimensionless time 1.

Figure 3. Analytical solution of dimensionless number distribution of a twocomponent aerosol system with pure growth following one-third order rates at dimensionless time 1 .

Figure 4. Numerical solution of dimensionless number distribution of a twocomponent aerosol system with pure growth following one-third order rates at dimensionless time 1 .

Figure 5. Analytical solution of dimensionless number distribution of a twocomponent aerosol system with pure evaporation following one-third order rates at dimensionless time 1.

Figure 6. Numerical solution of dimensionless number distribution of a twocomponent aerosol system with pure evaporation following one-third order rates at dimensionless time 1.

Figure 7. Analytical solution of dimensionless number distribution of a twocomponent aerosol system with mixed growth/evaporation following one-third order rates at dimensionless time 1 .

Figure 8. Numerical solution of dimensionless number distribution of a twocomponent aerosol system with mixed growth/evaporation following one-third order rates at dimensionless time 1 .

Figure 9. Analytical solution (solid line) and numerical solution (broken line) of dimensionless number distribution of a two-component aerosol system with pure coagulation at dimensionless time 1. (Hidden lines are removed). 
Figure 10 Analytical solution (solid line) and numerical solution (broken line) of dimensionless number distribution of a two-component aerosol system with simultaneous condensation and coagulation at dimensionless time 1 .

Figure 11. Numerical solution of dimensionless number distribution of a twocomponent aerosol system with simultaneous condensation and coagulation following one-third order condensation rates at dimensionless time 1 .

Figure 12. Comparison of direct solution, sectional solution, and analytical solution of a two-component aerosol system with pure condensation at dimensionless time 1. 


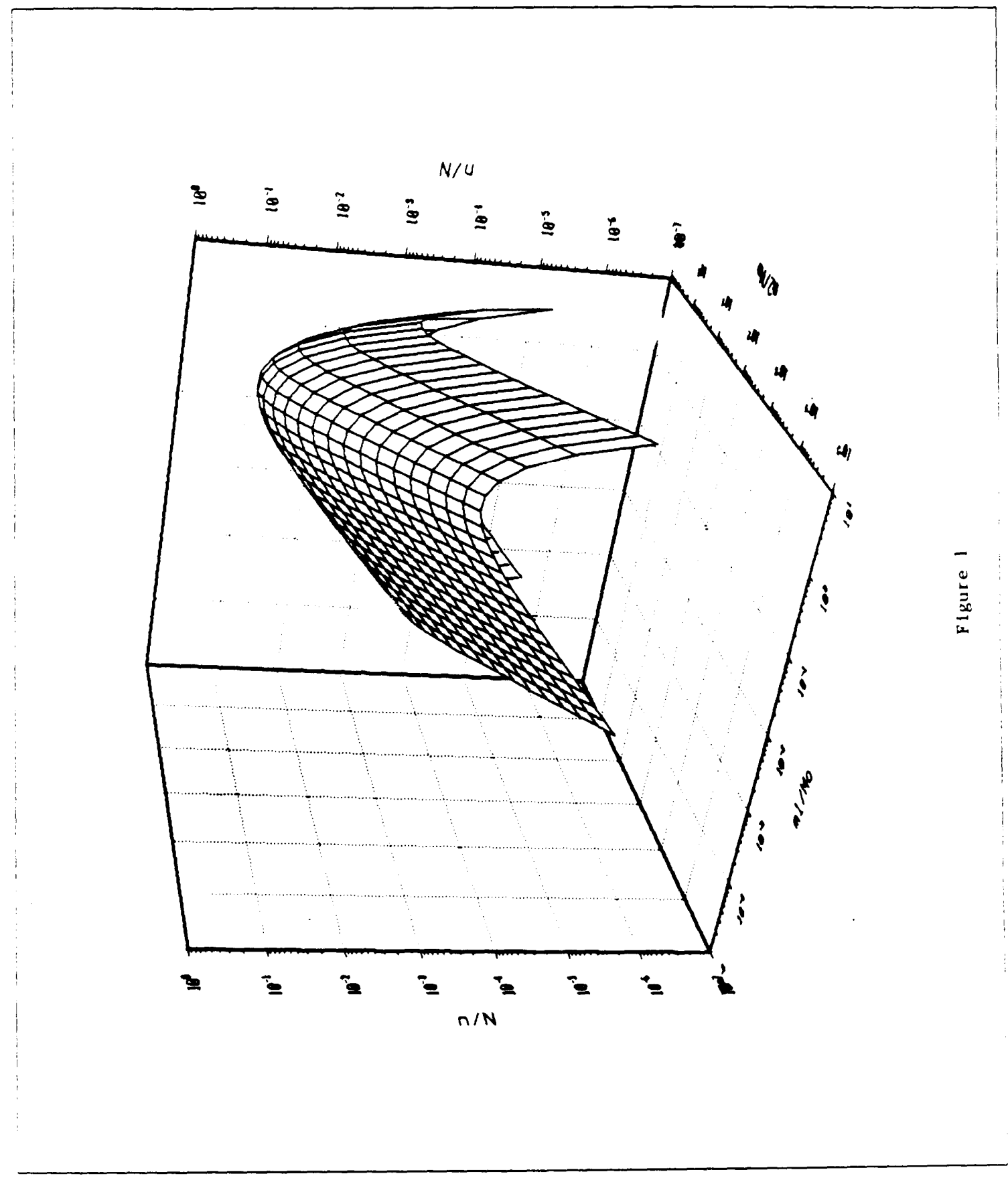




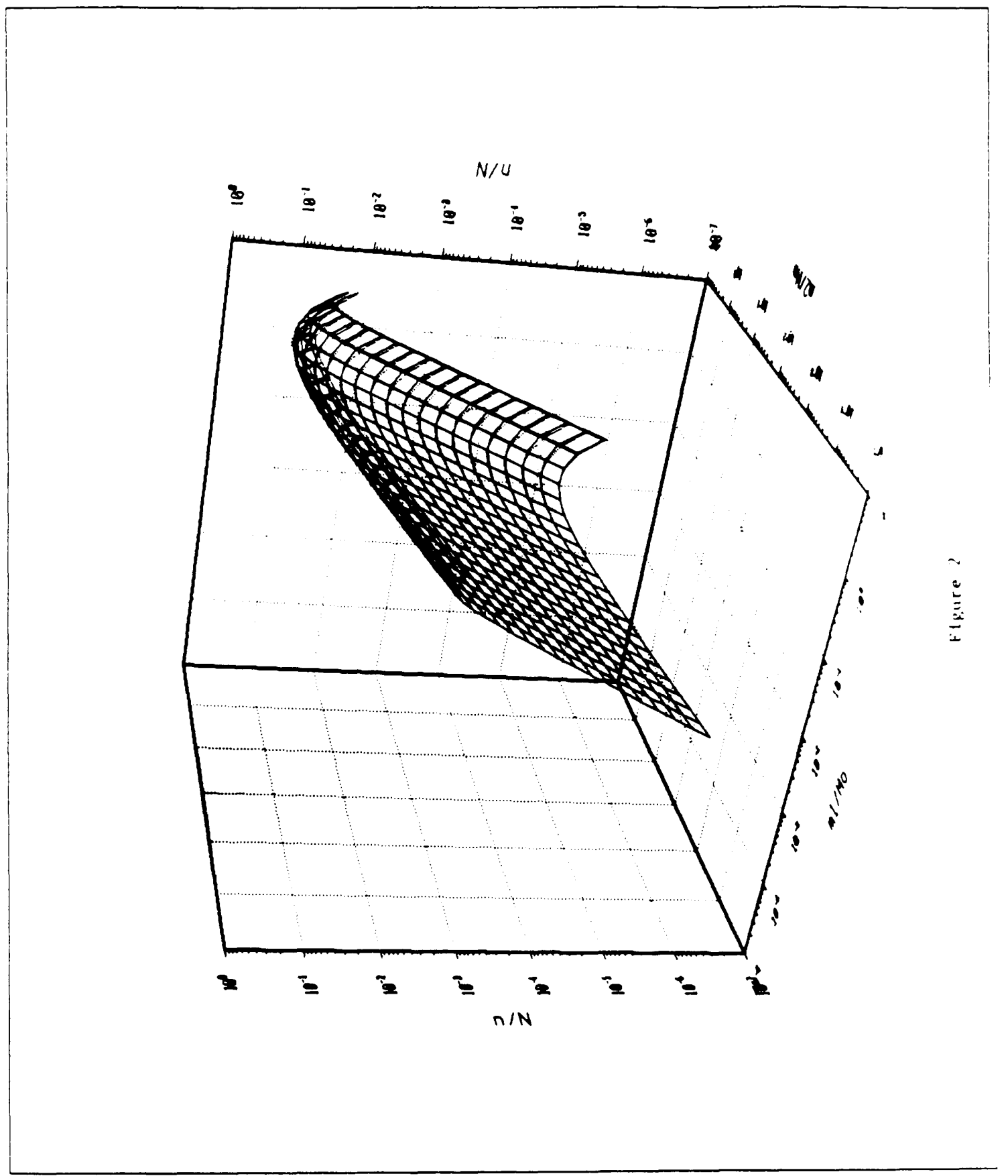




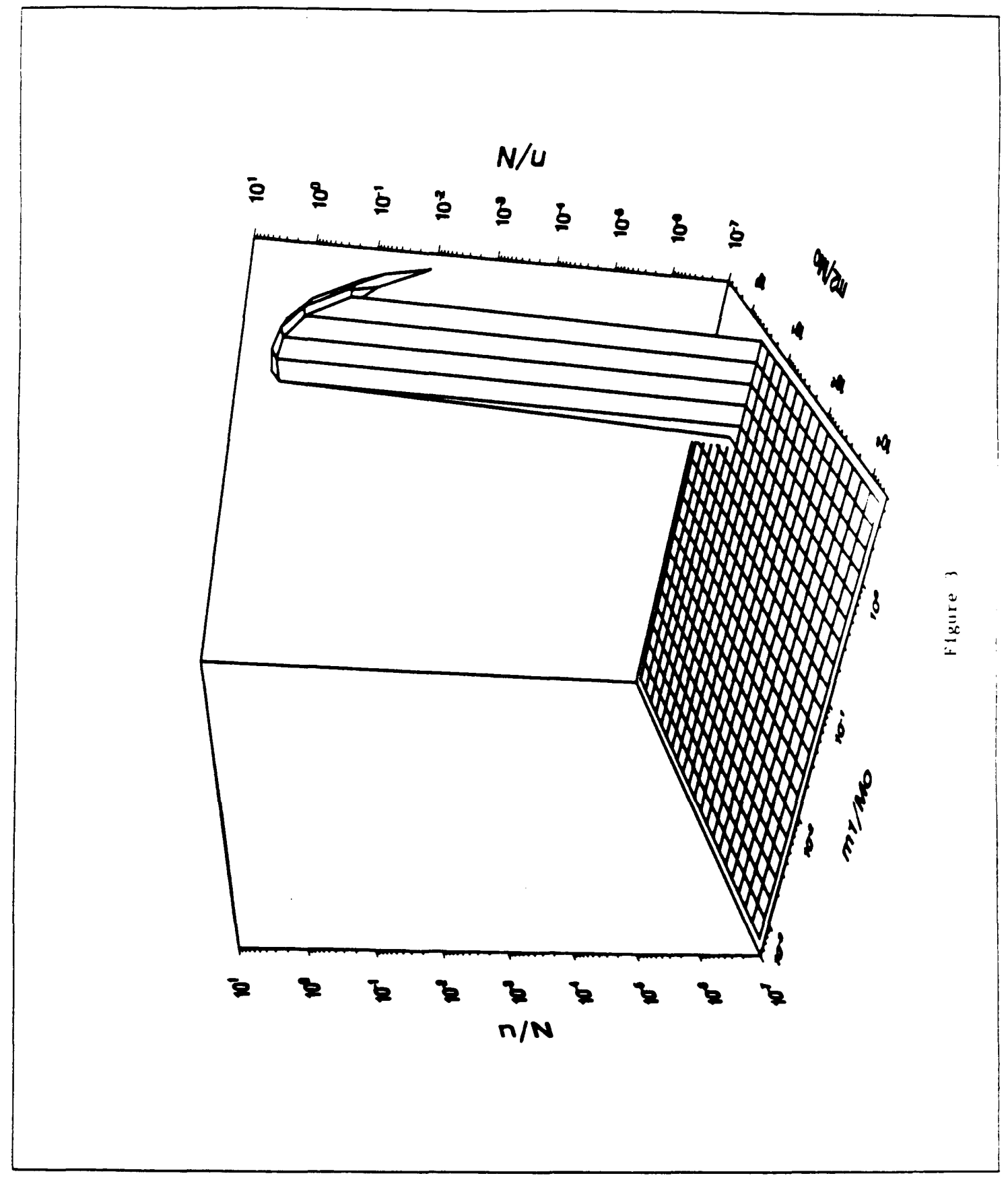




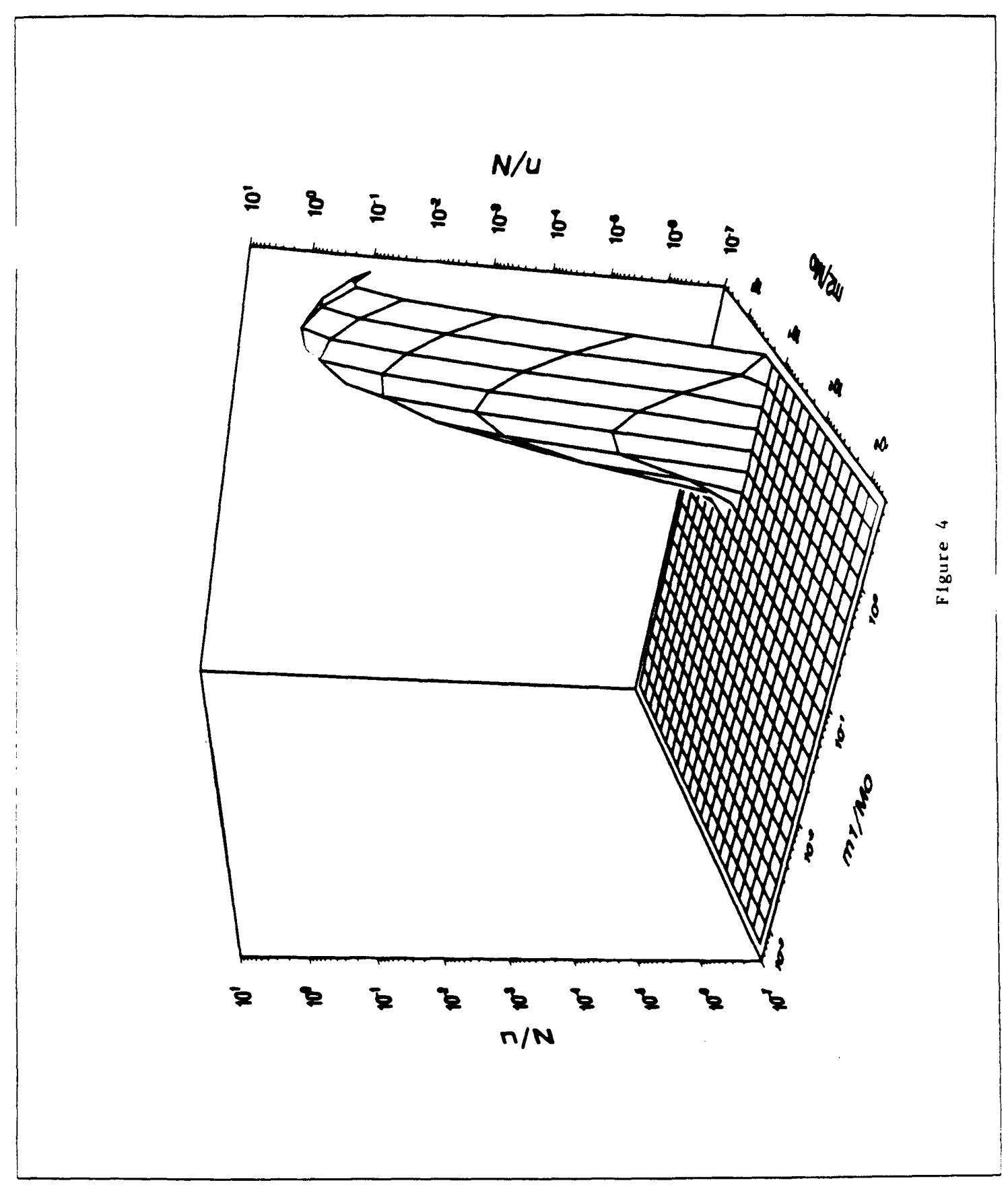




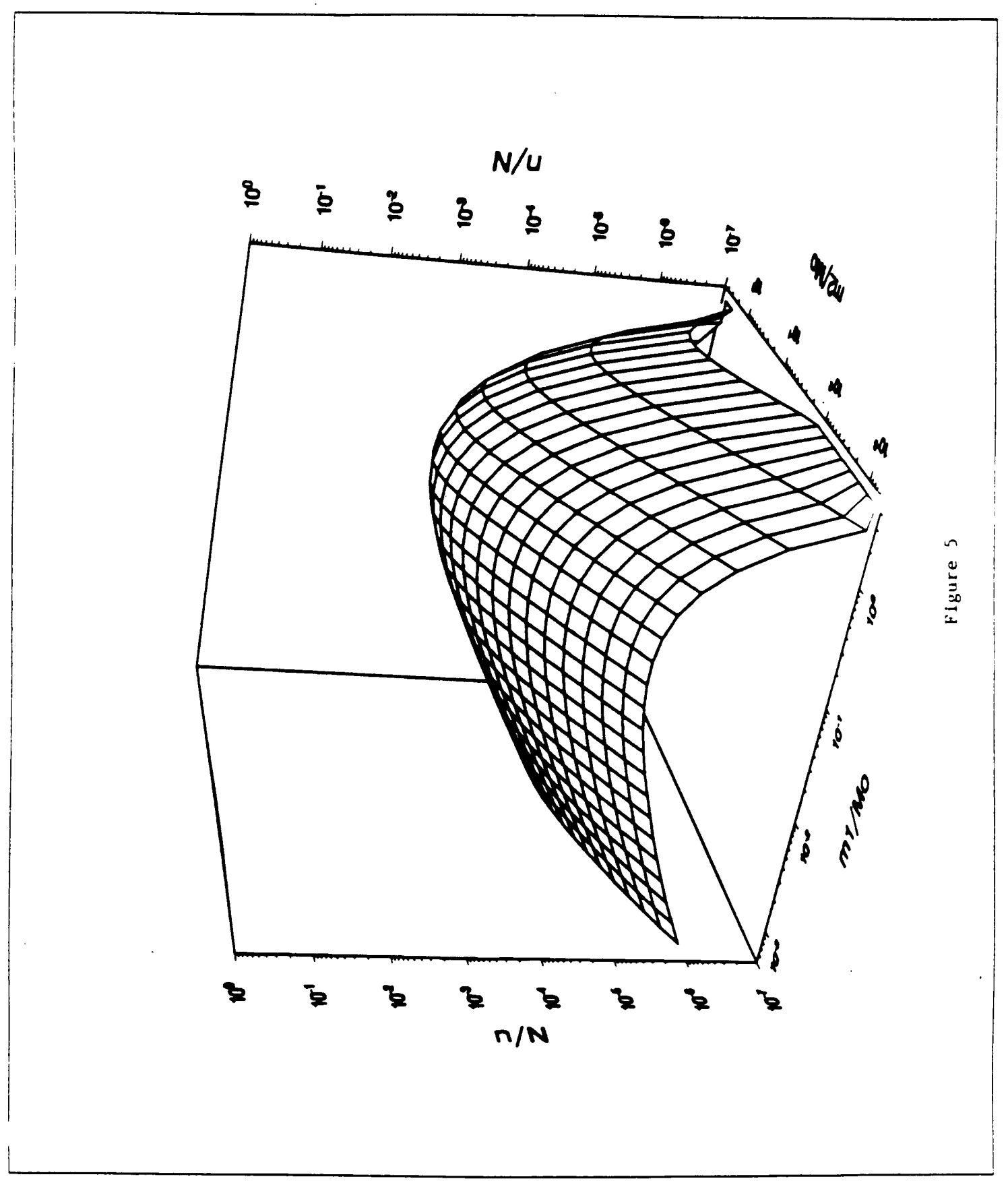




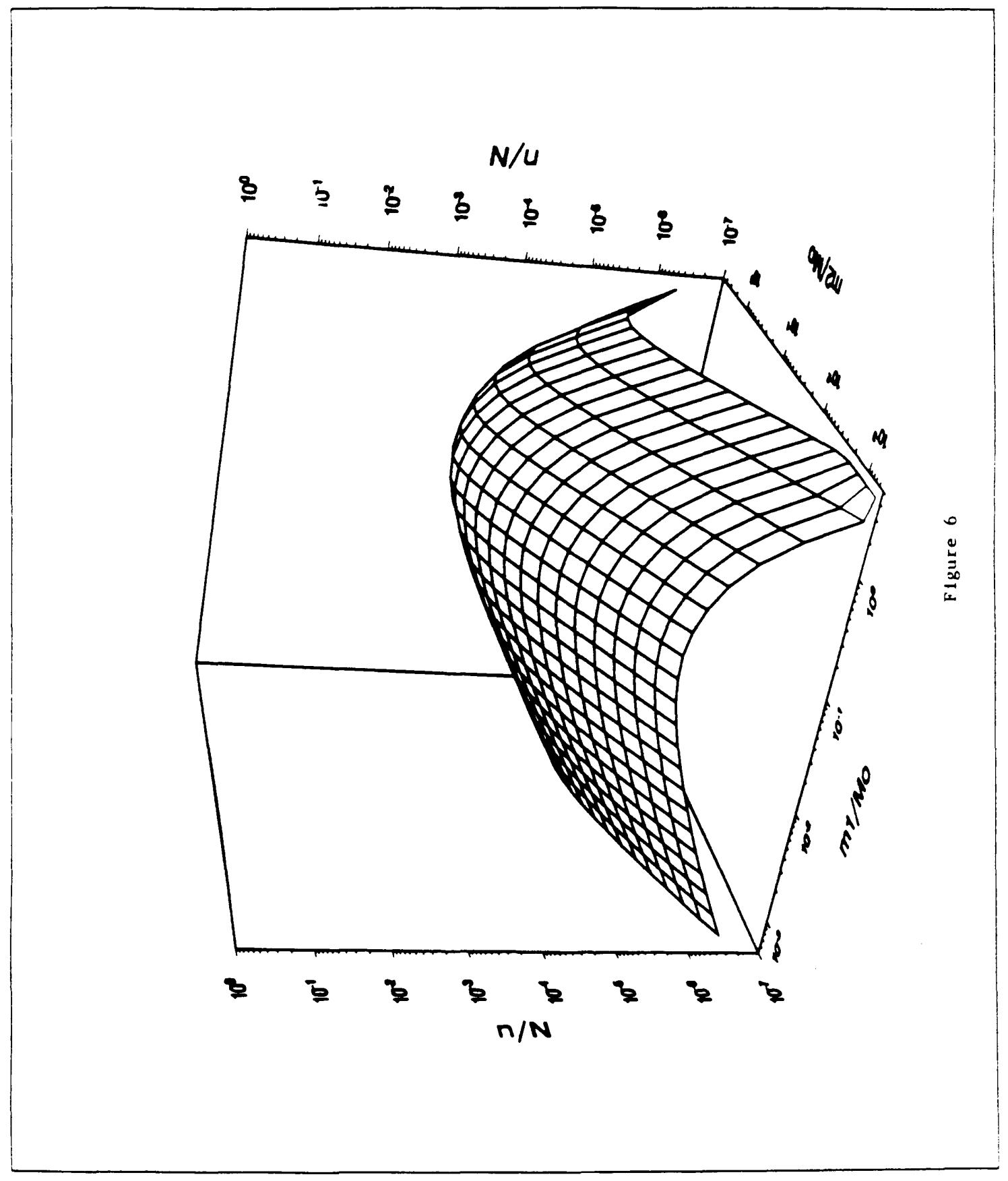




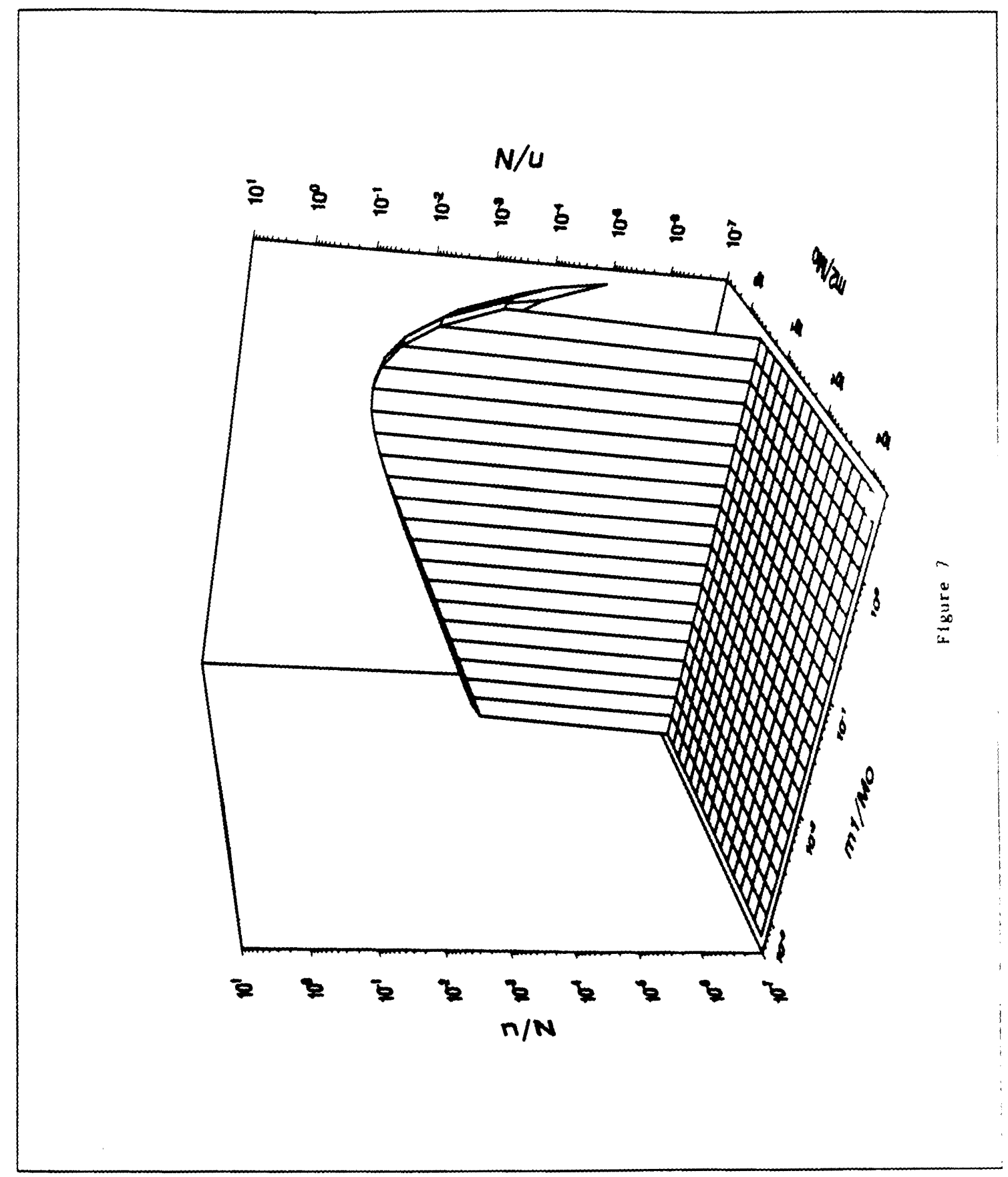




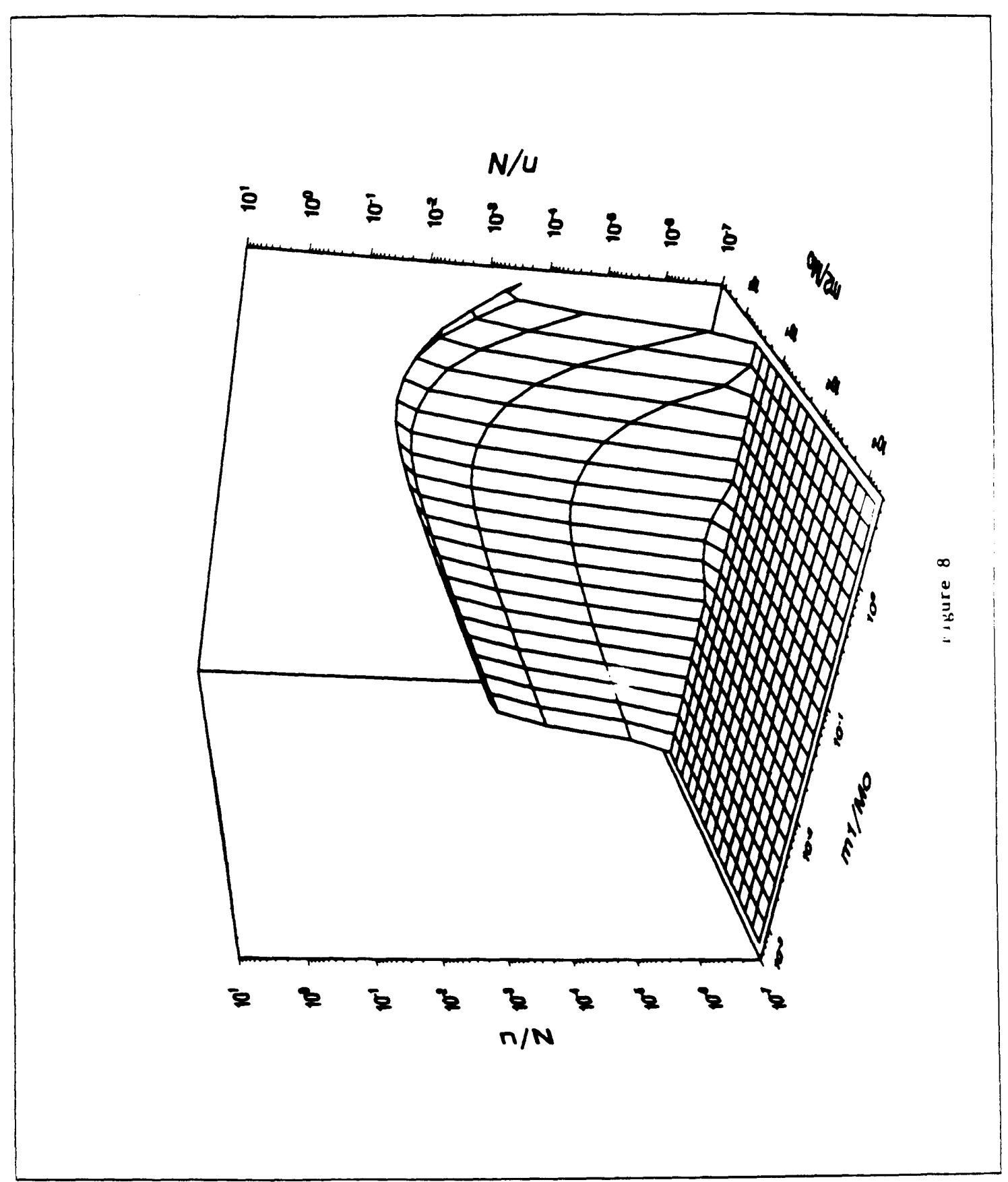




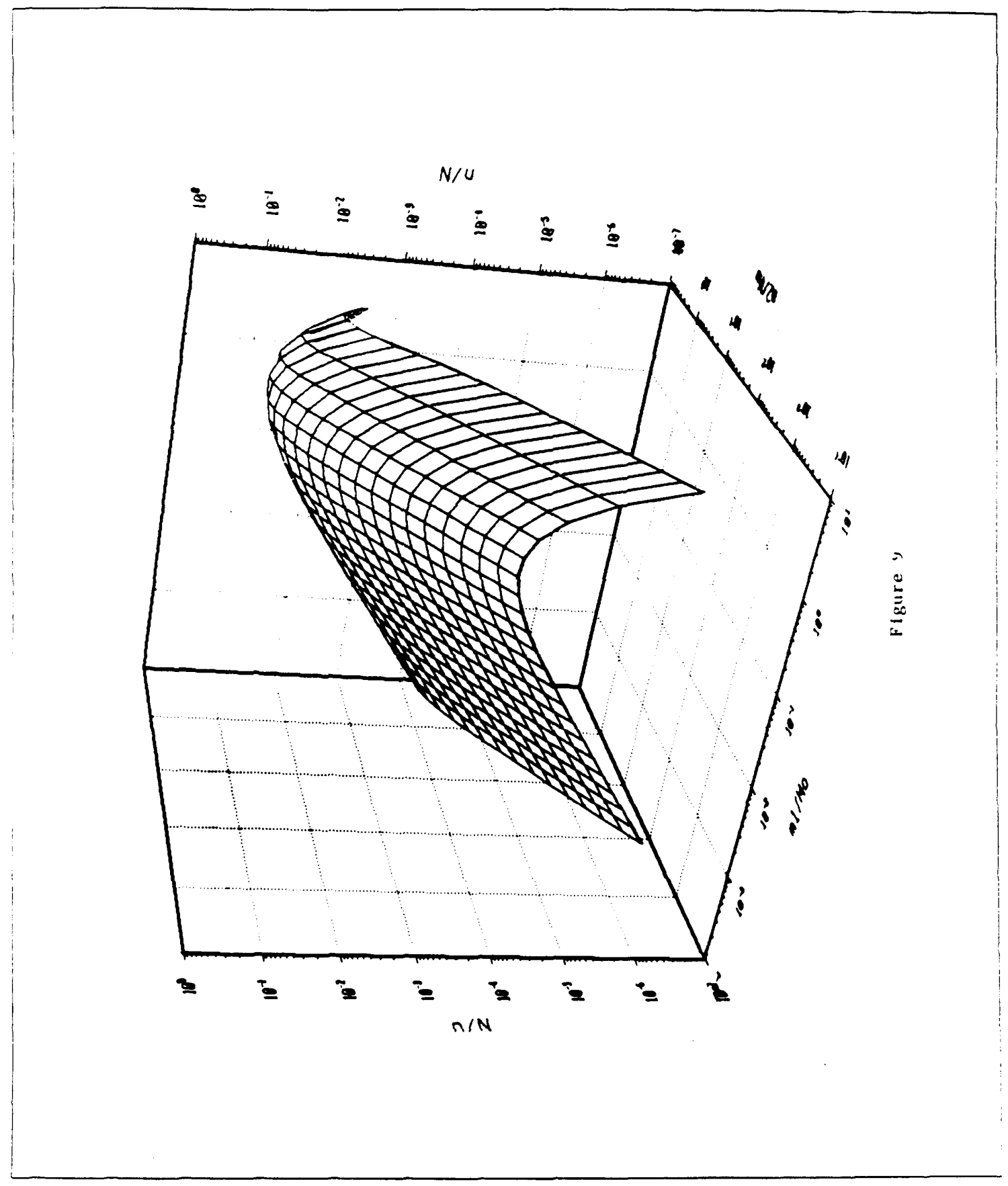




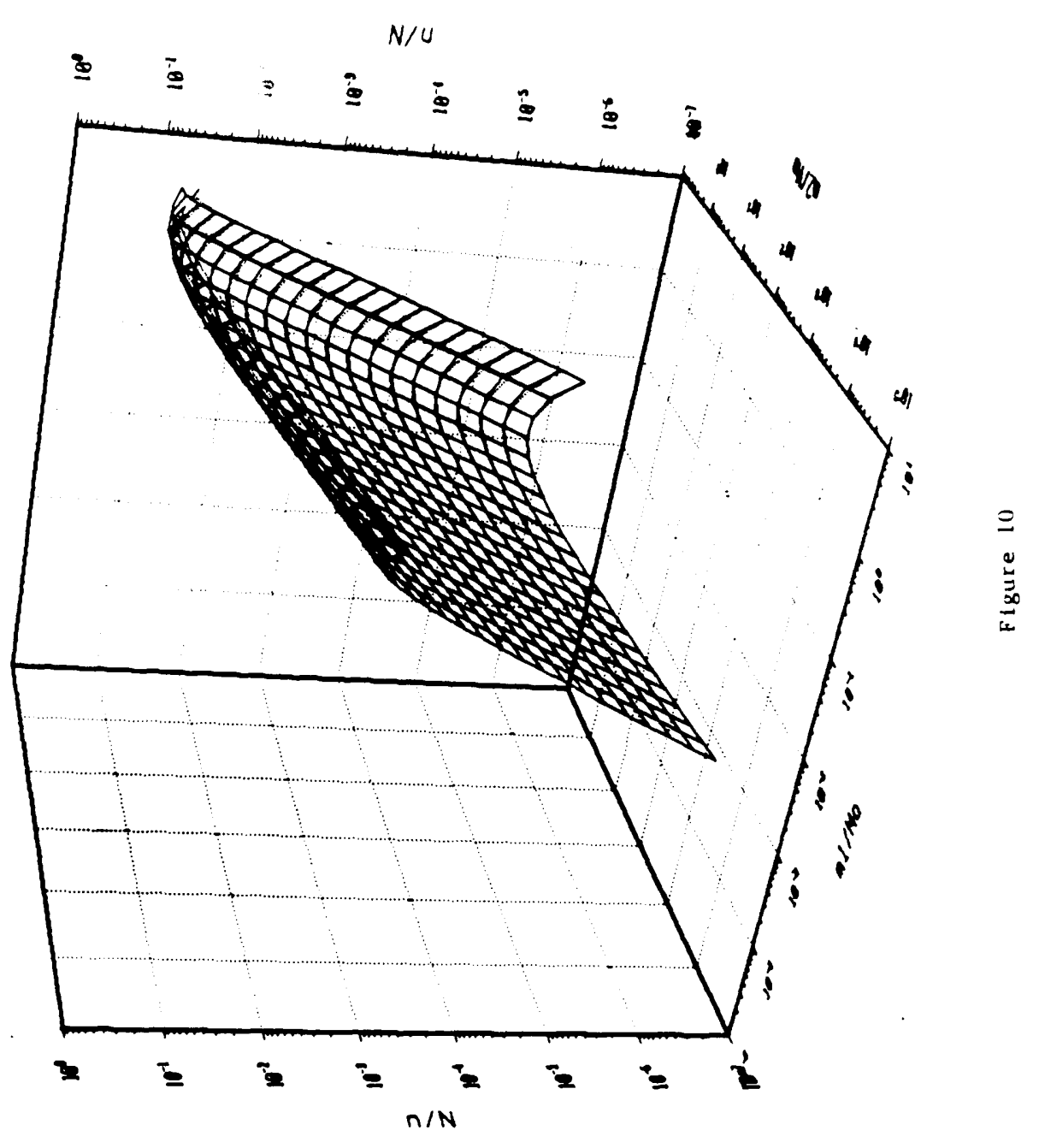




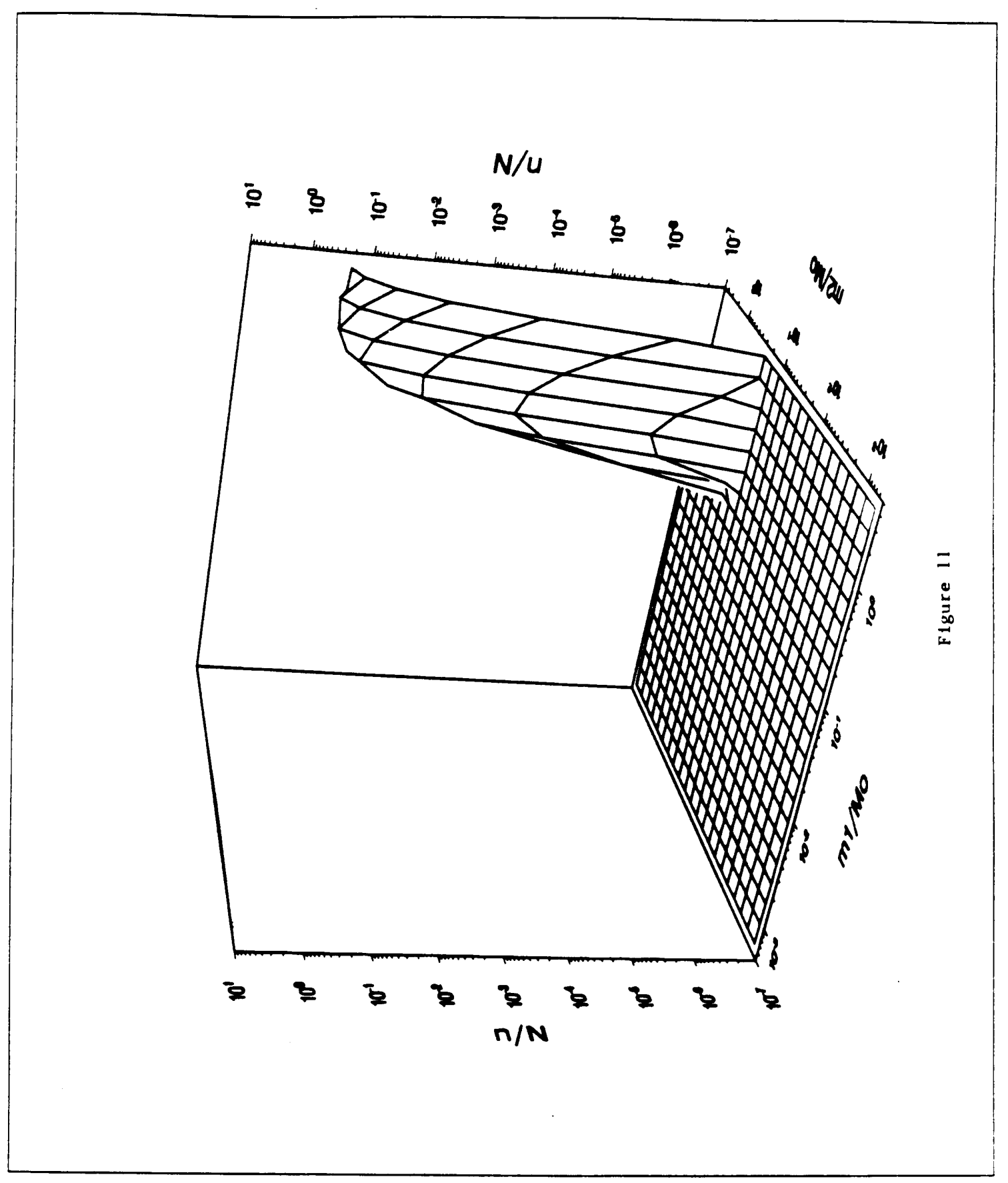




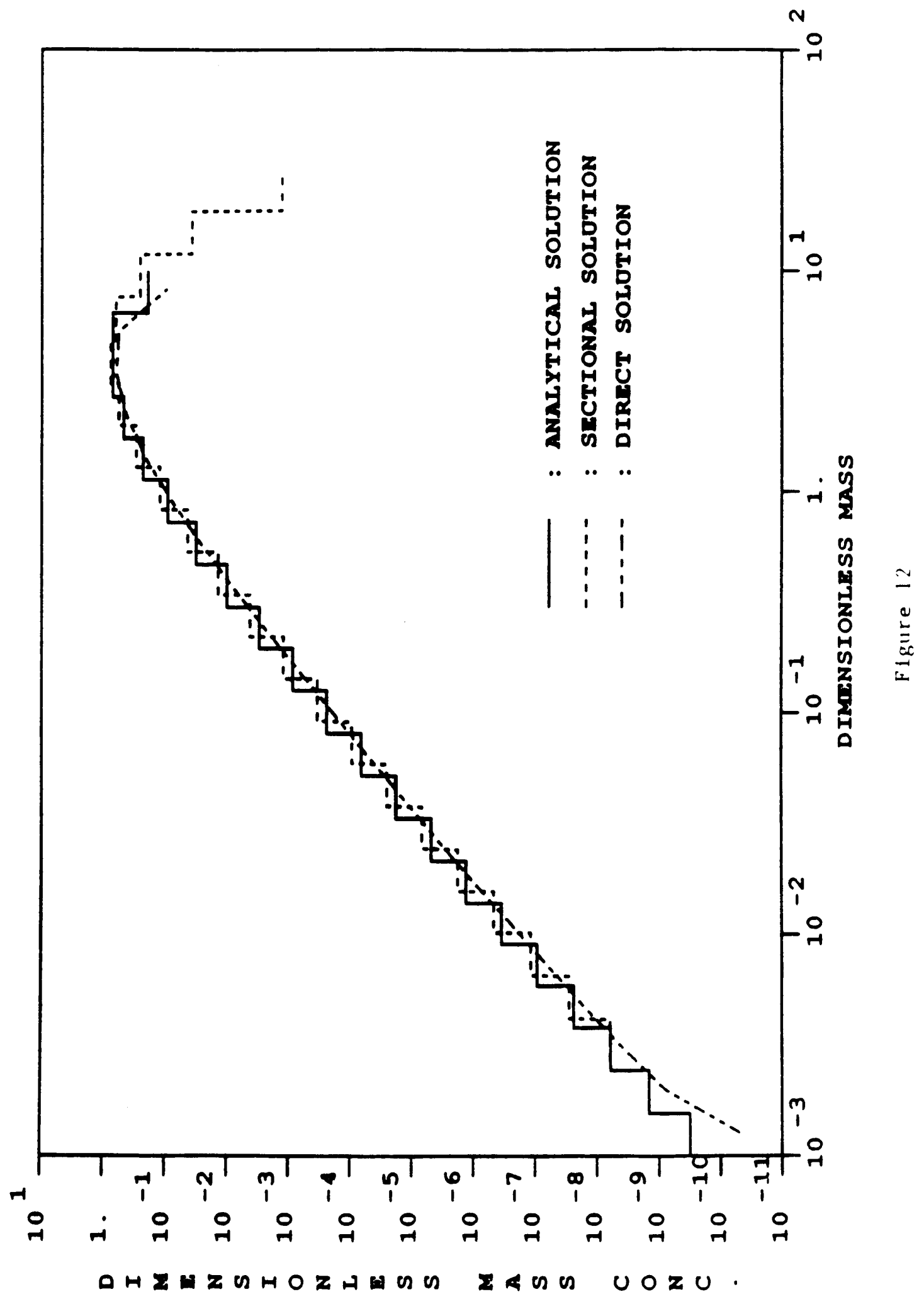




\section{Chapter IV}

USE OF

THE TANDEM DIFFERENTIAL MOBILITY ANALYZER TO EVALUATE THE DEGREE OF MIXING OF AEROSOL PARTICLES 


\section{INTRODUCTION}

Composition and/or property variations among aerosol particles of the same size can lead to different dynamical behavior. Junge (1963) introduced the concept of mixed particles, and Winkler (1973) described the two extremes of internal and external mixing. In an internally mixed aerosol all particles consist of the same relative mixture of substances, while in an externally mixed aerosol all particles consist individually of pure compounds. (See, for example, Heintzenberg and Covert, 1990). As an example, Figure 1 shows the effect of the degree of particle mixing on the dynamics of hygroscopic and non-hygroscopic particles of the same size. If the particles are internally mixed, all particles of the same size will grow equally when exposed to water vapor, while for an external mixture the non-hygroscopic and the hygroscopic particles exhibit different growth behavior. Another example is that with a refractive index difference among aerosol particles the sizes of equally sized light-absorbing and non-absorbing particles as inferred from an optical particle counter may be different (Covert et al., 1990).

The Tandem Differential Mobility Analyzer (TDMA), developed by Liu et al. (1978), (also called the aerosol mobility chromatograph) is an instrument consisting of two differential mobility analyzers (DMAs) separated by an aerosol conditioner. The essential feature of the TDMA is depicted in Figure 2. The size and/or the concentration of monodisperse particles screened by the first DMA (DMA1) from polydisperse feed particles can be changed in the conditioner and then measured by the second DMA (DMA2) and an aerosol concentration sensor. The TDMA is an ideal system to investigate the degree of mixedness of particles. Liu et al. (1978) and later McMurry and Stolzenburg (1989) demonstrated that particles of the same size can be separated according to their hygroscopicity difference. The TDMA has also been used to measure the evaporation rates of aerosol particles (Rader and McMurry, 1987), vapor pressure and surface free energy (Tao and McMurry, 1989), and in other applications in which aerosol size change can be induced (Stolzenburg and McMurry, 1988). 
Rader and McMurry (1986) developed a theoretical treatment of the TDMA system, which Stolzenburg and McMurry (1988) extended to include cases of more than one peak in the distribution and a spectrum of growth rates. In that treatment, the DMA1 was analyzed by the number-mobility distribution and the transfer function concept developed by Knutson and Whitby (1975) with approximations to describe the DMA1 exit distribution. To simulate the behavior of the particles in the conditioner, a least squares fitting procedure with three parameters for each distribution peak at the conditioner exit was proposed, one parameter for the particle wall loss and two others to describe the shape change of a peak inside the conditioner. If there are multiple peaks, the relative concentrations of each peak and the size changes can be calculated. A limitation of the geometric growth model is that it does not model the fundamental aerosol dynamic processes occurring in the conditioner.

An expanded approach to analyze the TDMA system is proposed here in which aerosol behavior in the conditioner is simulated by a recently developed routine for solving the multicomponent aerosol general dynamic equation (GDE) directly (Kim and Seinfeld, 1991). The GDE governing the number-composition distribution function, $n(\mathbf{m}, t)$, is

$$
\begin{aligned}
& \frac{\partial n(\mathbf{m}, t)}{\partial t}+\sum_{i=1}^{s} \frac{\partial}{\partial m_{i}}\left[I_{i}(\mathbf{m}, t) n(\mathbf{m}, t)\right] \\
& =\frac{1}{2} \int_{0}^{m_{1}} \cdots \int_{0}^{m_{\mathbf{s}}} \beta(\mathbf{u}, \mathbf{m}-\mathbf{u}) n(\mathbf{m}-\mathbf{u}, t) n(\mathbf{u}, t) d \mathbf{u} \\
& -n(\mathbf{m}, t) \int_{0}^{\infty} \cdots \int_{0}^{\infty} \beta(\mathbf{u}, \mathbf{m}) n(\mathbf{u}, t) d \mathbf{u} \\
& +R[\mathbf{m}, t, n(\mathbf{m}, t)]+S[\mathrm{~m}, t, n(\mathbf{m}, t)]
\end{aligned}
$$

where $m_{i}$ is the mass of the $i$-th component in a particle, and $\mathbf{m}$ is a vector of compositions $\left(m_{1}, \ldots, m_{s}\right)$, where $s$ is the total number of components. $n(\mathbf{m}, t) d \mathbf{m}$ is the number of particles having mass of component $i$, in the range $\left[m_{i}, m_{i}+d m_{i}\right]$ at time t. $I_{i}$ is the time rate change of the mass concentration of the $i$-th component from condensation/evaporation processes. $\beta(\mathbf{u}, \mathbf{m})=\beta(\mathbf{m}, \mathbf{u})$ is the binary coagulation coefficient. $R$ and $S$ are removal and source terms, respectively. 
In the numerical routine cited above no assumptions on the nature of the distribution are made, therefore, it is possible to simulate aerosol dynamics for arbitrarily mixed particles. Processes and mechanisms treated in the numerical routine are shown in Table 1.

The TDMA analysis approach is presented in the next section. Then, as an example, the experimental data of Liu et al. (1978) are simulated to demonstrate the approach.

\section{TDMA ANALYSIS}

The Differential Mobility Analyzer (DMA) (Knutson and Whitby, 1975) can select particles of a very narrow size range (practically monodisperse) from a polydisperse aerosol sample. Several inversion procedures to interpret DMA measurements have been proposed (Liu and Pui, 1974; Knutson, 1976; Hoppel, 1978; Haaf, 1980; Alofs and Balakumar, 1982; Kousaka et al., 1985; Wolfenbarger and Seinfeld, 1990; Reischl, 1991).

The behavior of a DMA is described by a so-called transfer function, the probability that a particle will leave via the sampling slit, which may be expressed in the form (Knutson and Whitby, 1975),

$$
\Omega=\left(\frac{1}{q_{a}}\right) \max \left[0, \min \left(q_{a}, q_{s},\left[\frac{1}{2}\left(q_{a}+q_{s}\right)-\left|\frac{2 \pi L V}{\ln \left(R_{2} / R_{1}\right)} Z_{p}+\frac{1}{2}\left(q_{m}+q_{c}\right)\right|\right]\right)\right]
$$

where $Z_{p}$ and $\Delta Z_{p}$ are the mean mobility and half-width of the transfer function,

$$
\begin{gathered}
Z_{p}=\frac{q_{c}+q_{m}}{4 \pi L V} \ln \left(\frac{R_{2}}{R_{1}}\right), \\
\Delta Z_{p}=\frac{\left(q_{a}+q_{s}\right)}{4 \pi L V} \ln \left(\frac{R_{2}}{R_{1}}\right)
\end{gathered}
$$

$L$ is the length of the DMA, $V$ is the applied rod voltage, $q_{c}, q_{m}, q_{s}$, and $q_{a}$ are the clean air, main outlet, sampling, and aerosol flow rates, respectively, and $R_{1}$ and $R_{2}$ are the inner radius of the DMA housing and the outer radius of the DMA center 
rod, respectively. The relation between mobility, $Z_{p}$, and diameter of a particle with $i$ elementary charges, $D_{p i}$, is

$$
\begin{gathered}
Z_{p}=\frac{i C_{d} e}{3 \pi \mu D_{p i}} \\
C_{d}=1+\mathrm{Kn}[1.37+0.4 \exp (-1.1 / \mathrm{Kn})]
\end{gathered}
$$

where $\mathrm{Kn}=2 \lambda / D_{p i}, C_{d}$ is the slip correction factor, $e$ is the charge of an electron, $1.6021 \times 10^{-19} \mathrm{C}, \mu$ is the gas viscosity, and $\lambda$ is the mean free path of the suspending gas. For a given applied voltage the mobility is single valued and fixed but the corresponding particle diameters are not. One can define a probability, $p\left(D_{p i}\right)$, of a particle having $i$ elementary charges with the diameter $D_{p i}$.

If the DMA 1 inlet number distribution is $\frac{d N_{o}}{d \ln D_{p}}$ or $n_{o}\left(\ln D_{p}\right)$, then the total number concentration, $N_{1}$, of the DMA1 output for a fixed voltage, $V$, can be represented by

$$
N_{1}=\int_{-\infty}^{\infty} \frac{q_{a 1}}{q_{s 1}} \sum_{i=1}^{\infty} p\left(D_{p i}\right) \Omega_{1} \frac{d N_{o}}{d \ln D_{p}} d \ln D_{p}
$$

For a sufficiently narrow transfer function, both $p\left(D_{p i}\right)$ and $\left.\frac{d N_{o}}{d \ln D_{p}}\right|_{D_{p}=D_{p i}}$ can be approximated as constants, then

$$
N_{1}=\frac{q_{a 1}}{q_{s 1}} \sum_{i=1}^{\infty}\left[p\left(D_{p i}\right) \frac{d N_{o}}{d \ln D_{p i}}\right] \int_{-\infty}^{\infty} \Omega_{1} d \ln D_{p}
$$

By changing the integration variable in Eq. (8),

$$
\int_{-\infty}^{\infty} \Omega_{1} d \ln D_{p}=\int_{0}^{\infty} \frac{\Omega_{1}}{Z_{p 1}}\left(\frac{d D_{p i} / D_{p i}}{d Z_{p 1} / Z_{p 1}}\right) d Z_{p}
$$

From Eq. (5),

$$
\begin{aligned}
\left(\frac{d D_{p i} / D_{p i}}{d Z_{p 1} / Z_{p 1}}\right) & =\left(\frac{1}{D_{p i}} \frac{C_{d}}{D_{p i}}\right) /\left(\frac{d\left(C_{d} / D_{p i}\right)}{d D_{p i}}\right) \\
& \equiv \Phi_{1}
\end{aligned}
$$

Thus, Eq. (7) becomes

$$
N_{1}=\frac{q_{a 1}}{q_{s 1}} \sum_{i=1}^{\infty}\left[p\left(D_{p i}\right) \frac{d N_{o}}{d \ln D_{p i}}\right] \int_{0}^{\infty} \frac{\Omega_{1}}{Z_{p 1}} \Phi_{1} d Z_{p} .
$$

In the conventional DMA analysis, the entire number distribution, $\frac{d N_{o}}{d \ln D_{p}}$, is obtained, so the accuracy of the constant approximations can be evaluated. Also the values of 
$\frac{d N_{o}}{d \ln D_{p i}}$ are obtained and thus the multiple charging effect can be assessed. Since, however, in the DMA1 analysis, $\frac{d N_{o}}{d \ln D_{p i}}$ is not known, the singly charged particle sizes can be calculated but the total number concentration and the relative contribution of the multiple charging effect are not known.

The effect of multiple charging and the accuracy of the approximation that the DMA1 inlet distribution is constant over a narrow transfer function are important issues, both of which can be addressed if the initial distribution is known. For some cases, however, this is not possible. As will be shown in Section 3, the effect of multiple charging for small particles (usually less than $0.1 \mu \mathrm{m}$ ) can be negligible when $D_{p 2}$ is on the right side of a unimodal peak. If the particles screened by the DMA1 lie near the peak of the distribution, the constant distribution assumption is a reasonable approximation.

The distribution at the conditioner exit is determined by the DMA2 and the subsequent aerosol concentration sensor. Following the same approach used in the DMA1 analysis, let the DMA2 inlet or the conditioner outlet number distribution be $\frac{d N_{r}}{d \ln D_{p}}$ or $n_{r}\left(\ln D_{p}\right)$, then the total number concentration, $N_{2}$, of the DMA2 output aerosol particles for a voltage, $V$, is

$$
N_{2}=\int_{-\infty}^{\infty} \frac{q_{a 2}}{q_{s 2}} \sum_{i=1}^{\infty} p\left(D_{p i}\right) \Omega_{2} \frac{d N_{r}}{d \ln D_{p}} d \ln D_{p}
$$

Then Eq. (12) finally becomes

$$
N_{2}=\frac{q_{a 2}}{q_{s 2}} \sum_{i=1}^{\infty}\left[p\left(D_{p i}\right) \frac{d N_{r}}{d \ln D_{p i}}\right] \int_{0}^{\infty} \frac{\Omega_{2}}{Z_{p 2}} \Phi_{2} d Z_{p} .
$$

Now, both the inlet and outlet conditions between the two DMAs are fully described except for the total number concentration of the conditioner inlet particles and the effect of multiple charging. The differences in size and/or concentration between the two distributions depend on the dynamic process(es) in the conditioner. The multicomponent aerosol GDE (Kim and Seinfeld, 1991) will be used to simulate the process(es) occurring in the conditioner.

As inherent feature of the TDMA is that the aerosol number concentration at the conditioner inlet is generally not known. One cannot use the number concentration at 
the conditioner exit obtained by the DMA2 as that at the conditioner inlet since nonnumber conserving process(es), such as coagulation, deposition, or nucleation, may be occurring inside the conditioner. Since there may be particle losses that cannot be modeled, an equipment specific deposition parameter will be retained.

\section{APPLICATION OF THE TDMA TO DETERMINE AEROSOL MIXEDNESS}

The TDMA application on which we focus here is to evaluate the degree of mixedness of an aerosol sample. Liu et al. (1978) demonstrated that the TDMA can separate particles of the same size but different composition according to their hygroscopicity differences. Aqueous solution droplets of $\mathrm{K}_{2} \mathrm{SO}_{4}$ and $\mathrm{H}_{2} \mathrm{SO}_{4}$ were dried at a relative humidity (R.H.) of about $8 \%$, introduced into DMA1, and then humidified to about $53 \%$ R.H. in the conditioner. At this relative humidity, the $\mathrm{H}_{2} \mathrm{SO}_{4}$ solution particles grew while those of $\mathrm{K}_{2} \mathrm{SO}_{4}$ solution did not. The post-condensation size distribution was measured by the DMA2. The measured number distributions are shown in Figure 3. The solid curve corresponds to the case of no humidification between the two DMAs and the broken curve is that of humidification. Therefore, the solid curve represents the screened narrow size distribution of the DMA1 modified by the attenuation due to particle losses. The experimental data are chosen as a model example to be simulated by the new TDMA analysis approach.

\subsection{Data Inversion}

Since Liu et al.'s (1978) experimental data are represented as electric current vs. DMA2 rod voltage, the data must be first inverted to a widely used format, i.e., number distribution vs. diameter or mass.

STEP 1: Convert the applied DMA2 rod voltage to mobility and finally to diameter.

The relation between the mobility transfer function and operating conditions is given in Eq. (5) and the relation between mobility and particle diameter is given in 
Eq. (7). The dimension of the DMAs and the operating conditions are those of Liu and Pui (1974). Since the length of the DMA was not stated in Liu and Pui (1974), the value reported in Knutson and Whitby (1975) is used in the data inversion. All other dimensions of the DMA in the two reports are the same. Since particles of different sizes can have the same mobility due to multiple charging, a spectrum of diameters of particles with the same mobility was calculated.

STEP 2: Convert the electric current to particle number concentration.

If all particles are assumed to be singly charged, the relation between the current and the apparent number concentration is $N_{s}=\frac{I}{q e}$, where $q$ is the aerosol flow rate entering the electrometer current sensor. Liu et al. (1978) considered all particless detected as being singly charged based on the fact that for particles in Boltzmann equilibrium multiple charging does not become a significant factor until the particl. diameters exceed $0.1 \mu \mathrm{m}$. Since Kousaka et al. (1985) subsequently showed that the Fuchs equilibrium satisfies experimental data better than the Boltzmann equilibrium, a further analysis of the possibility of multiple charging is warranted.

Let $N_{i}$ be the number concentration of particles with $i$ elementary charges and mobility, $Z_{p}$, then the true number concentration is

$$
N=\sum_{i=1}^{\infty} N_{i}
$$

The apparent number concentration, $N_{s}$, is

$$
N_{s}=\sum_{i=1}^{\infty} i N_{i} .
$$

Combining the above two equations,

$$
\frac{N}{N_{s}}=\frac{\sum_{i=1}^{\infty} N_{i}}{\sum_{i=1}^{\infty} i N_{i}} .
$$

The relation between $N_{i}$ and $N_{1}$ can be expressed as,

$$
N_{i}=p\left(D_{p i}\right) f_{i} \frac{1}{p\left(D_{p 1}\right)} N_{1}
$$


where $p\left(D_{p i}\right)$ is defined in section 2 in the text before Eq. (7) and $f_{i}$ is the ratio of the number distribution at $D_{p i}$ to that at $D_{p 1}$. Then Eq. (16) becomes

$$
\frac{N}{N_{s}}=\frac{p\left(D_{p 1}\right)+\sum_{i=2}^{\infty} p\left(D_{p i}\right) f_{i}}{p\left(D_{p 1}\right)+\sum_{i=2}^{\infty} i p\left(D_{p i}\right) f_{i}}
$$

The values of $p\left(D_{p 1}\right)$ and $p\left(D_{p i}\right)$ can be obtained from the Fuchs theory. From Kousaka et al. (1985), the ranges of the numerical values in the size range of interest are $2 \times 10^{-3}-1.5 \times 10^{-2}$ for $p\left(D_{p 2}\right)$ and $0.1-0.18$ for $p\left(D_{p 1}\right)$. The probability of a particle being more than doubly charged diminishes drastically, so is not considered here. Therefore, unless the values of $f_{2}$ are very large, the assumption of Liu et al. (1978) that all the particles in this size range of interest are singly charged is a valid one. The difficulty is that $\frac{d N_{0}}{d \ln D_{p i}}$ and the $f_{i}$ are unknown since the DMA1 inlet distribution was not obtained. So let us consider under what condition $f_{2}$ is a large number. For a unimodal distribution, the usual case, $f_{2}$ is large only if $D_{p 2}$ is on the left side of the peak, and multiply charged particles of $\mathrm{K}_{2} \mathrm{SO}_{4}$ solution would not grow, not affecting the overall dynamics. On the other hand, multiply charged particles of $\mathrm{H}_{2} \mathrm{SO}_{4}$ solution would affect the dynamics; some grow beyond the measuring range, while others grow but remain in the measuring range but cannot be detected since there is no information about the initial distribution.

Although we cannot assess the effect of multiple charging quantitatively, the effect is expected to be small and the number concentration was calculated based on this approximation.

STEP 3: Convert the number concentration to number distribution.

Based on the above calculations, the actual number distribution vs. the particle diameter is calculated. Since the size range is very narrow, it is reasonable to assume the number distribution in the narrow size range is constant (Stolzenburg and McMurry, 1988; Reischl, 1991). With this assumption, the number distribution is obtained by dividing the number concentration in one size by the size range.

\subsection{Thermodynamic Properties}


The aerosol particles in Liu et al.'s experiment (1978) consist of three species, $\mathrm{K}_{2} \mathrm{SO}_{4}, \mathrm{H}_{2} \mathrm{SO}_{4}$, and $\mathrm{H}_{2} \mathrm{O}$. It is assumed that $\mathrm{K}_{2} \mathrm{SO}_{4}$ and $\mathrm{H}_{2} \mathrm{SO}_{4}$ particles are externally mixed. From the data, it is deduced that about $32 \%$ of the initial aerosol particles consisted of $\mathrm{K}_{2} \mathrm{SO}_{4}-\mathrm{H}_{2} \mathrm{O}$ with the remainder being $\mathrm{H}_{2} \mathrm{SO}_{4}-\mathrm{H}_{2} \mathrm{O}$. It is assumed that at the final stage the particles in the larger peak consist of $\mathrm{H}_{2} \mathrm{SO}_{4}-\mathrm{H}_{2} \mathrm{O}$ and the particles in the smaller peak consist of $\mathrm{K}_{2} \mathrm{SO}_{4}-\mathrm{H}_{2} \mathrm{O}$. It is also assumed that the ambient temperature and pressure are $298 \mathrm{~K}$ and $1 \mathrm{~atm}\left(1.01325 \times 10^{5} \mathrm{~Pa}\right)$. The effect of these assumptions will be discussed later.

The relation between the partial pressure of $i$-th component in the gas phase and the composition in the aqueous aerosol phase is given by,

$$
\begin{aligned}
p_{i, b} & =\gamma_{i} x_{i} p_{i}^{o} \\
& =a_{i} p_{i}^{o}
\end{aligned}
$$

where the subscript $i$ denotes the $i$-th component. $\gamma_{i}$ is the activity coefficient. $a_{i}$ is the activity, $x_{i}$ is the mole fraction in the aqueous aerosol phase, and $p_{i, b}$ and $p_{i}^{0}$ are the partial pressure on the bulk flat surface and the saturated vapor pressure (equilibrium pressure), respectively. The Kelvin effect may be significant for ultrafine particles,

$$
\frac{p_{i}}{p_{i, b}}=\ln \left(\frac{4 \pi \sigma \overline{v_{i}}}{R T D_{p}}\right)
$$

where $p_{i}$ and $p_{i, b}$ are the vapor pressures over the droplet and over a flat bulk surface, respectively. $\sigma$ is the surface tension, $\overline{v_{i}}$ is the molar liquid volume of species $i, R$ is the gas constant $(8.314 \mathrm{~J} /$ mole $\mathrm{K})$, and $I$ is the absolute temperature.

The thermodynamic properties needed to calculate the initial and the final particle compositions of an $\mathrm{H}_{2} \mathrm{SO}_{4}-\mathrm{H}_{2} \mathrm{O}$ system were obtained from a numerical routine by Kreidenweis-Dandy (1989). The saturation vapor pressure of sulfuric acid was calculated from the relation given by Ayers et al. (1980) and properties of $\mathrm{K}_{2} \mathrm{SO}_{4}-\mathrm{H}_{2} \mathrm{O}$ system were obtained from Lobo (1984). The initial and the final states of the $\mathrm{H}_{2} \mathrm{SO}_{4}-\mathrm{H}_{2} \mathrm{O}$ particles are assumed to be in equilibrium with the ambient conditions. This enables the calculation of the mass fractions of the initial and the final 


\section{$\mathrm{H}_{2} \mathrm{SO}_{4}-\mathrm{H}_{2} \mathrm{O}$ aerosols.}

Since thermodynamic property data for a ternary systems are scarce, and therefore the approach used here is to assume the system is an ideal solution.

\subsection{Data Preparation for the Numerical Routine}

The experimental data were presented as number concentration $(N)$ vs. DMA voltage, equivalent to particle diameter $\left(D_{p}\right)$. Thus, one can calculate the numbersize distribution $\frac{d N}{d \log D_{p}}$, or $n\left(\log D_{p}, t\right)$ directly from the expc. imental data. Since the numerical routine is based on multiple composition variables, the required number distribution must be in the form of $\frac{d N}{d \ln m_{1} \ldots d \ln m_{s}}$, or $n\left(\ln m_{1}, \ldots, \ln m_{s}\right)$, where $s$ is the number of components. To obtain the required distribution, it is assumed that the number distribution inside each grid is uniform.

During the experiment, the aerosol particles consist of either $\mathrm{K}_{2} \mathrm{SO}_{4}-\mathrm{H}_{2} \mathrm{O}$ or $\mathrm{K}_{2} \mathrm{SO}_{4}-\mathrm{H}_{2} \mathrm{O}$, but not a combination. In the numerical routine, the logarithmic transformation precludes two-component aerosol particles in an three-component system. A practical solution is to assume an infinitesimal amount of the third component. The amount of $\mathrm{K}_{2} \mathrm{SO}_{4}$ and $\mathrm{H}_{2} \mathrm{SO}_{4}$ assumed present in the $\mathrm{H}_{2} \mathrm{SO}_{4}-\mathrm{H}_{2} \mathrm{O}$ and $\mathrm{K}_{2} \mathrm{SO}_{4}-\mathrm{H}_{2} \mathrm{O}$ aerosols, respectively, was $0.1 \%$ of the minimum amount of $\mathrm{K}_{2} \mathrm{SO}_{4}$ and $\mathrm{H}_{2} \mathrm{SO}_{4}$ in the existing particles. That amount of $\mathrm{H}_{2} \mathrm{SO}_{4}$ in a $\mathrm{K}_{2} \mathrm{SO}_{4}-\mathrm{H}_{2} \mathrm{O}$ particle is too small to affect the thermodynamic properties of the particle since the activity of $\mathrm{H}_{2} \mathrm{SO}_{4}$ in a water solution of that concentration is about $1.9 \times 10^{-16}$. Likewise the amount of $\mathrm{K}_{2} \mathrm{SO}_{4}$ assumed in a $\mathrm{H}_{2} \mathrm{SO}_{4}-\mathrm{H}_{2} \mathrm{O}$ particle does not change the thermodynamic properties. The mass contribution of the artificially added compounds was less than $0.02 \%$ of the total mass concentration.

\subsection{Numerical Results}

The DMA2 outlet without humidification is used as the DMA1 outlet distribution since both total number concentrations differ by only about $2 \%$; the dominant process is hygroscopic growth. Also by doing so, the parameter that accounts for the particle 
depositional loss can be set as 1.0 since both data were measured at the DMA2.

Figure 4 shows the initial distributions obtained by the experiment and the numerical inputs with and without the Kelvin effect. Note that the numerical distribution compared in the figures is based on a one-dimensional size variable and therefore is obtained by integrating the distribution based on the multidimensional composition variables

$$
\begin{aligned}
& n\left(D_{p}\right)=\sum_{i, j, k} \Theta \int_{m_{i, 1}} \int_{m_{2, j}} \int_{m_{3, k}} n\left(m_{1}, m_{2}, m_{3}\right) d m_{3} d m_{2} d m_{1} \\
\Theta= & 1 \quad \text { if } \quad D_{p}-\Delta D_{p} \leq D_{p}\left(m_{1, i}+m_{2, j}+m_{3, k}\right) \leq D_{p}+\Delta D_{p} \\
= & 0 \quad \text { otherwise } \quad i, j, k=1, \ldots, n s
\end{aligned}
$$

where $n s$ is the number of grids for each component. The numerical distributin... clearly represent the experimental data well. Also, note that the Kelvin effect can \&... neglected.

The simulation is terminated when the ratio of the mass concentration of the simulated result to that of the initial distribution becomes the same as that measured. Without the Kelvin effect, the required time for the particles to reach the final state was about $3.75 \mu$ s increasing to $4.1 \mu$ s with the Kelvin effect. This is expected since the Kelvin effect increases the water concentration at the particle surface increase, decreasing the concentration difference.

Figures 5 and 6 show the predicted size distributions calculated with two different numerical methods, the repeated upwind difference method (RUD) and the TaylorGalerkin method (TGFEM), respectively. Both methods predict the peak heights accurately. For the larger peak, the RUD predicted about 95 and $97 \%$ of the experimental peak height while the TGFEM predicted about 96 and $101 \%$ of that depending on whether the Kelvin effect is included. The TGFEM is slightly better than the RUD in predicting the peak height accurately here, but the RUD has been found to perform better than previous applications (Tsang and Rao, 1988, 1990; Chock, 1990). 
Note that both the methods overpredicted the smaller peak height. Also note that the position of the simulated peaks lie at slightly smaller sizes than those measured by about $0.001 \mu \mathrm{m}$ for the particles of $\mathrm{K}_{2} \mathrm{SO}_{4}$ solution (smaller peak) and slightly larger than that by about $0.001 \mu \mathrm{m}$ for the particles of $\mathrm{H}_{2} \mathrm{SO}_{4}$ solution (larger peak). Though small, the discrepancies of the peak positions are real. The overprediction of the smaller peak may be caused by the assumption that the two sulfur compounds were totally unmixed. Apparently, particles of the two solutions were mixed, dried, and then sent through the aerosol charger. If some coagulation might have occurred, a simple analysis (Pui, private communication) shows that the residence time before DMA1 was comparable to the characteristic time of coagulation. These mixed particles are classified either as pure $\mathrm{K}_{2} \mathrm{SO}_{4}$ solution which could not grow numerically but grows experimentally, or particles of pure $\mathrm{H}_{2} \mathrm{SO}_{4}$ solution which grew faster numerically than the real particles. The overall effect of a fraction of the particles that may be intermediately mixed in the numerical simulation is the undergrowth of the assumed pure $\mathrm{K}_{2} \mathrm{SO}_{4}$ solution particles and the overgrowth of the assumed pure $\mathrm{H}_{2} \mathrm{SO}_{4}$ solution particles. The measured distributions are consistent with this explanation. In Figure 2, one can see that the smaller peak position with the hygroscopic growth is larger than that without the growth. Thus, even particles of pure $\mathrm{K}_{2} \mathrm{SO}_{4}$ (assumed) were observed to grow somewhat which can be reasonably explained by the mixed particles concept.

The effect of other processes, evaporation, coagulation, and deposition was also studied. A slight evaporation of $\mathrm{H}_{2} \mathrm{SO}_{4}$ was predicted but the amount was negligible. The characteristic time of coagulation in the conditioner was about $5 \times 10^{4} \mathrm{~s}$. Also, the rate of mass removal by deposition was about thirteen orders of magnitude less than that by condensation. Therefore, it is concluded that condensation was the only substantial process occurring in the conditioner.

\section{CONCLUSION}


An analysis of the TDMA system is presented based on the multicomponent aerosol GDE allowing the simulation of an arbitrarily mixed aerosol. The data of Liu et al. (1978) are simulated to demonstrate the ability of the new TDMA analysis approach.

\section{ACKNOWLEDGMENTS}

The authors thank R. C. Flagan, B. Wyslouzil, and D. Y.H. Pui for helpful discussions. This work was supported by National Science Foundation Grant ATM-9003186. 


\section{REFERENCES}

Alofs, D.J., and Balakumar, P. (1982). J. Aerosol Sci. 13:513-527.

Ayers, G.P., Gillett, R.W., and Gras, J.L. (1980). Geophys. Res. Lett. 7:433-436.

Chock, D.P. (1990). Atmos. Environ., 24A:1-19.

Covert, D.S., Heintzenberg, J., and Hansson, H.-C. (1990). Aerosol Sci. Technol. 12:446-456.

Haaf, W. (1980). J. Aerosol Sci. 11:189-200.

Heintzenberg, J., and Covert, D.S. (1990). J. Atmos. Chem. 10:383-397.

Hoppel, W.A. (1978). J. Aerosol Sci. 9:41-54.

Junge, C.E. (1963). Air Chemistry and Radioactivity. Academic Press, New York.

Kim, Y.P., and Seinfeld, J.H. (1991). J. Colloid Interface Sci. submitted.

Knutson, E.O. (1976). In Fine Particles (B.Y.H. Liu ed.). Academic Press, New York, pp 740-762.

Knutson, E.O., and Whitby, K.T. (1975). J. Aerosol Sci. 6:443-451.

Kousaka, Y., Okuyama, K., and Adach, M. (1985).Aerosol Sci. Technol. 4:209225.

Kreidenweis-Dandy, S. (1989). Ph. D. Thesis. California Institute of Technology, Pasadena.

Liu, B.Y.H., and Pui, D.Y.H. (1974). J. Colloid Interface Sci. 47:155-171.

Liu, B.Y.H., Pui, D.Y.H., Whitby, K.T., Kittelson, D.B., Kousaka, Y., and McKenzie, R.L. (1978). Atmos. Environ. 12:99-104.

Lobo, V.M.M. (1984). Electrolyte Solutions: Literature Data on Thermodynamics and Transport Properties. Coimbra.

McMurry, P.H., and Stolzenburg, M.R. (1989). Atmos. Environ. 23;497-507.

Rader, D.J., and McMurry, P.H. (1986). J. Aerosol Sci. 17:771-787.

Rader, D.J., and McMurry, P.H. (1987). Aerosol Sci. Technol. 6:247-260.

Reischl, G.P. (1991). Aerosol Sci. Technol. 14:5-24.

Stolzenburg, M.R., and McMurry, P.H. (1988). TDMAFIT User's Manual, PTL 
Publication No. 653. University of Minnesota, Minneapolis.

Tao, Y., and McMurry, P.H. (1989). Environ. Sci. Technol. 23:1519-1523.

Tsang, T.H., and Rao, A. (1988). Aerosol Sci. Technol. 9:271-277.

Tsang, T.H., and Rao, A. (1990). Int'l J. Numer. Meth. in Fluids. 10:753-769.

Winkler, P. (1973). J. Aerosol Sci. 4:373-387.

Wolfenbarger, J.K., and Seinfeld, J.H. (1990), J. Aerosol Sci. 21:227-247. 
Table 1. Processes and Mechanisms Treated in Numerical Routine

$\begin{array}{lll}\text { Process } & \text { Mechanism }\end{array}$

Scheme

Condensation/

Evaporation

Coagulation

Deposition

Source

Simultaneous

Processes
Diffusion-controlled

(Maxwell relation),

Volume reaction-controlled

gas-to-particle conversion

Brownian coagulation

Linear deposition

User given

Pre-specified

User given

Product type

Gaussian quatiature

With Coagulution

With coagulatwn

With coagulation

Operator splitting
Repeated upwind

difference,

Taylor-Galerkin 


\section{FIGURE CAPTION}

Figure 1. An illustration of the effect of particle mixedness on aerosol dynamics: Hygroscopicity difference affects internally and externally mixed particles differently.

Figure 2. Schematic diagram of the TDMA system.

Figure 3. Measured size distributions of Liu et al. (1978), converted to number distribution vs. particle diameter. Solid line; without growth, broken line; with growth.

Figure 4. Initial distribution. Solid line; measured size distribution without growth, single dotted line; the numerical initial distribution without the Kelvin effect. double dotted line; the numerical initial distribution with the Kelvin effect.

Figure 5. Final distribution, the numerical results using the repeated upwind difference method. Solid line; measured size distribution with growth, single dotted line; the numerical result without the Kelvin effect, double dotted line; the numerical result with the Kelvin effect.

Figure 6. Final distribution, the numerical results using the Taylor-Galerkin method. Solid line; measured size distribution with growth, single dotted line; the numerical result without the Kelvin effect, double dotted line; the numerical result with the Kelvin effect. 
HYGROSCOPICITY: INTERNAI VS. EXTERNAI MIXTURE

INTERNAI MIXTURT
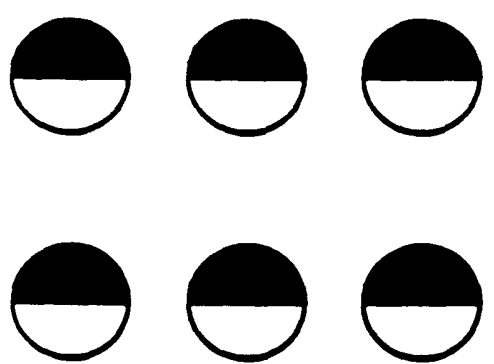

EXTERALI MIXTURS
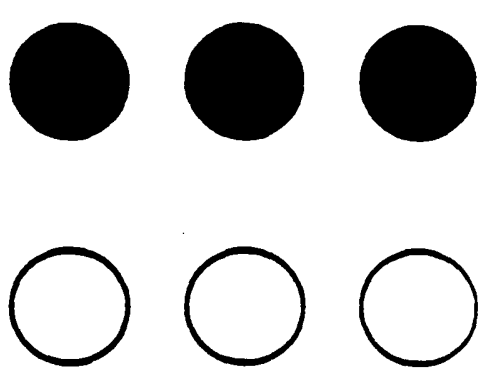
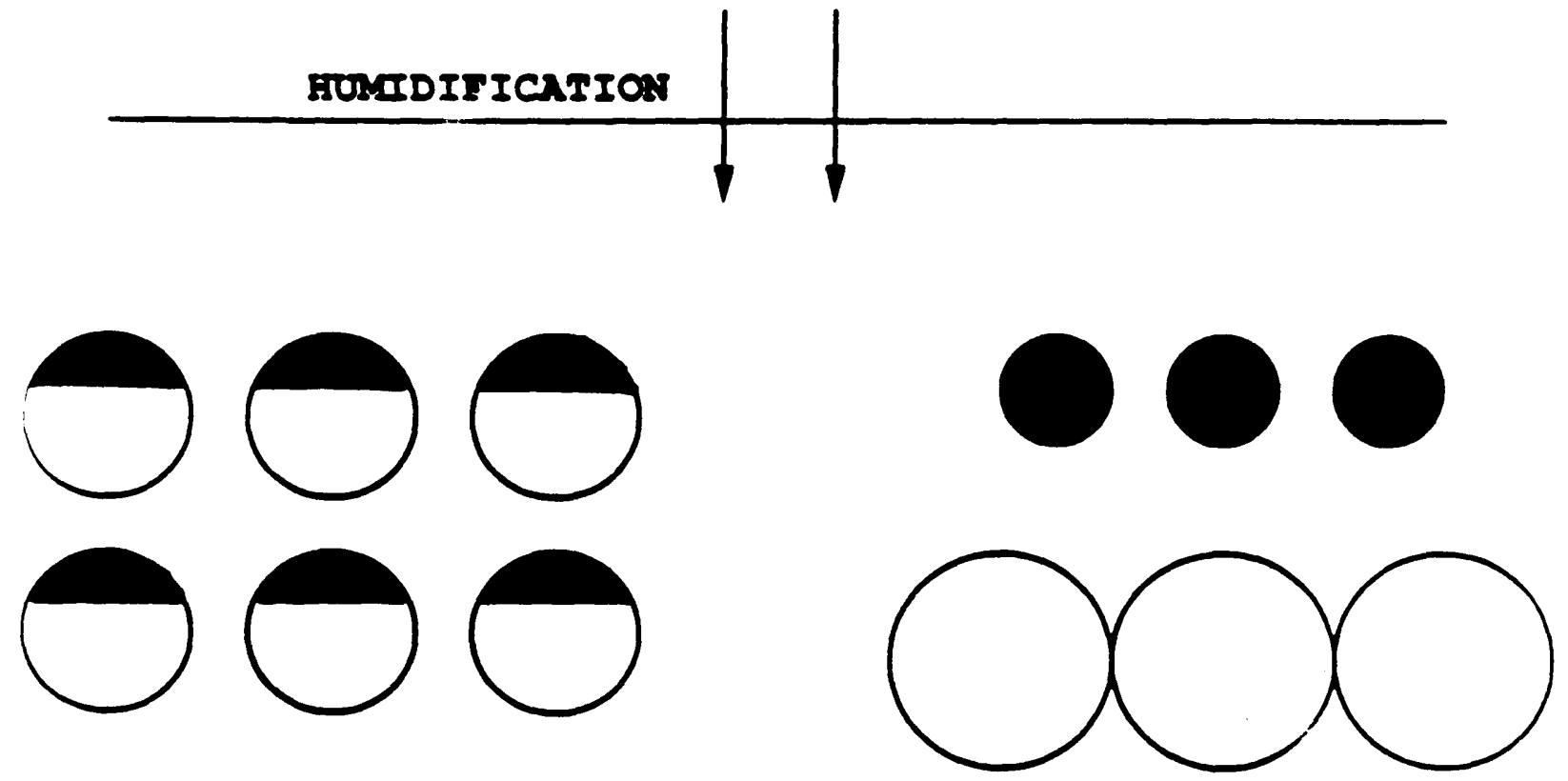

Figure 1 


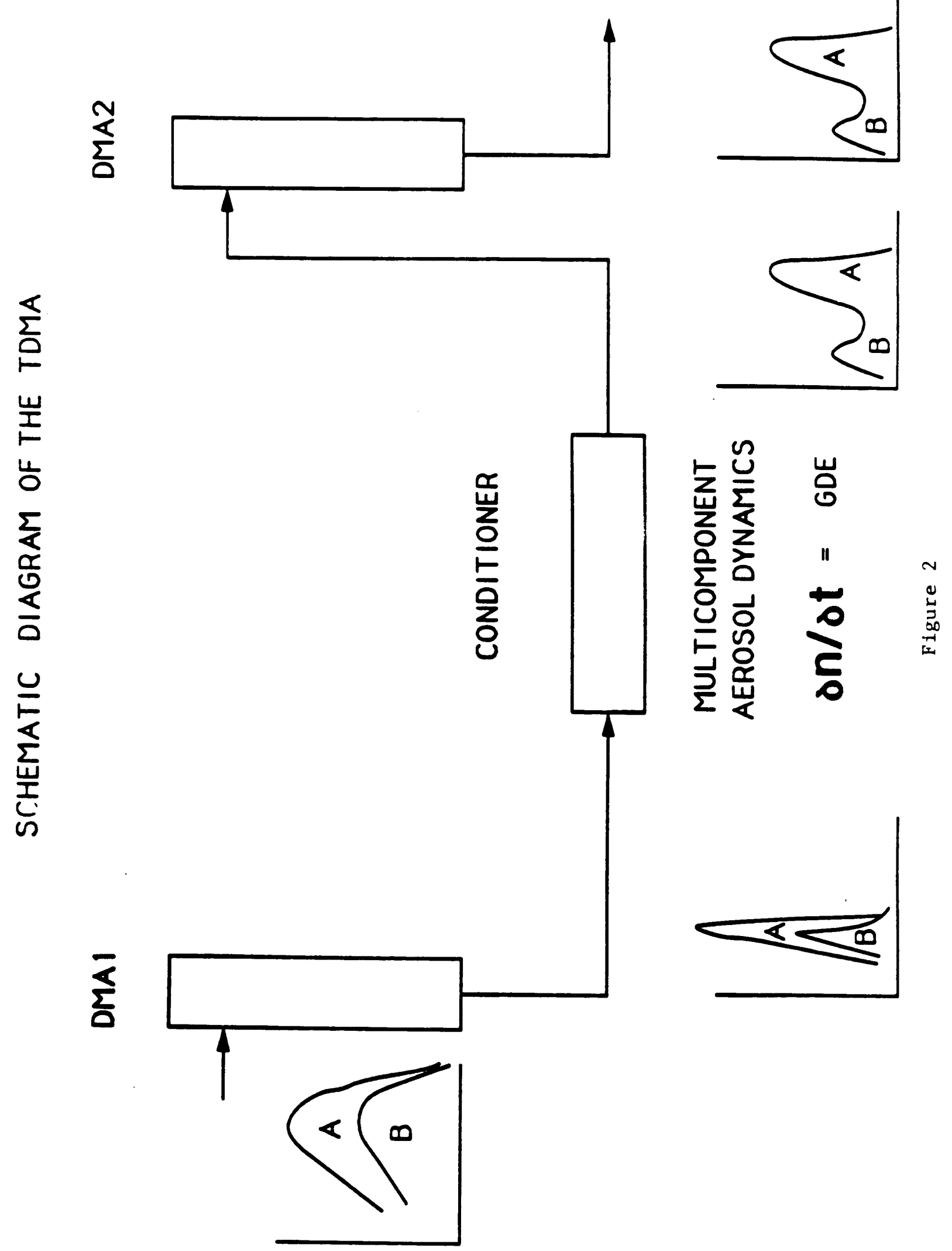




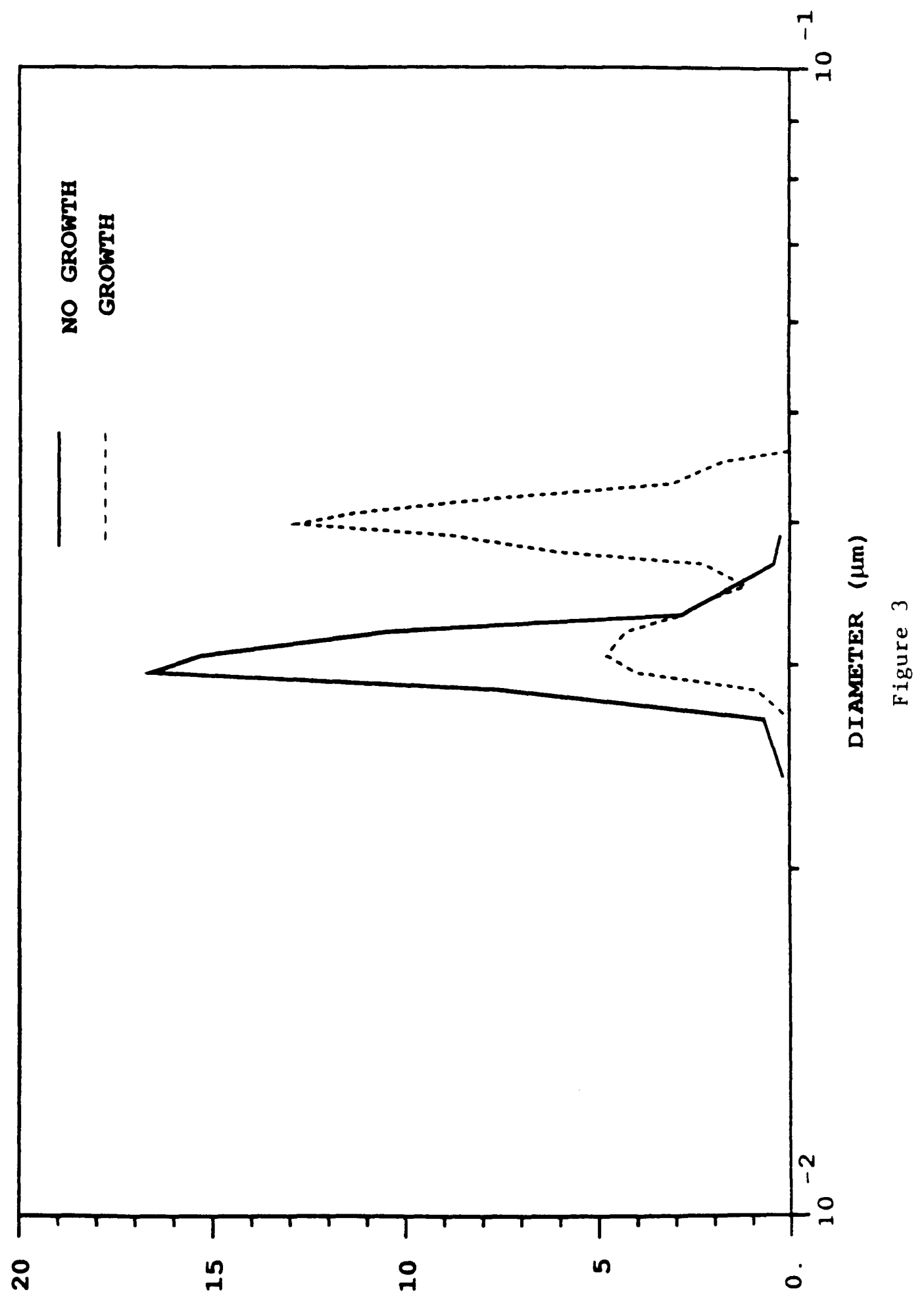

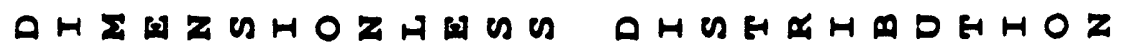




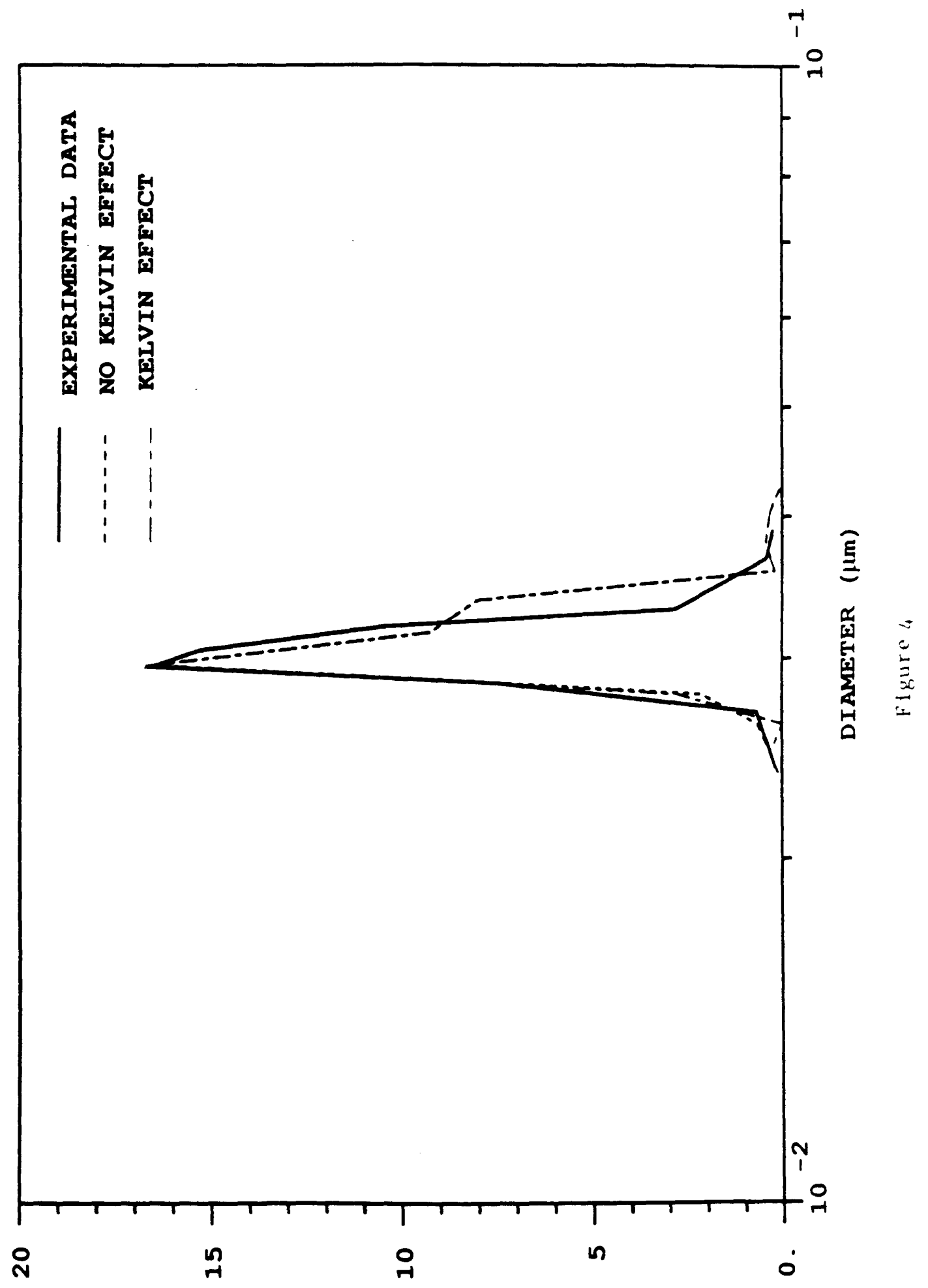

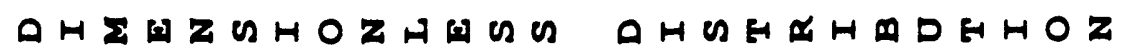




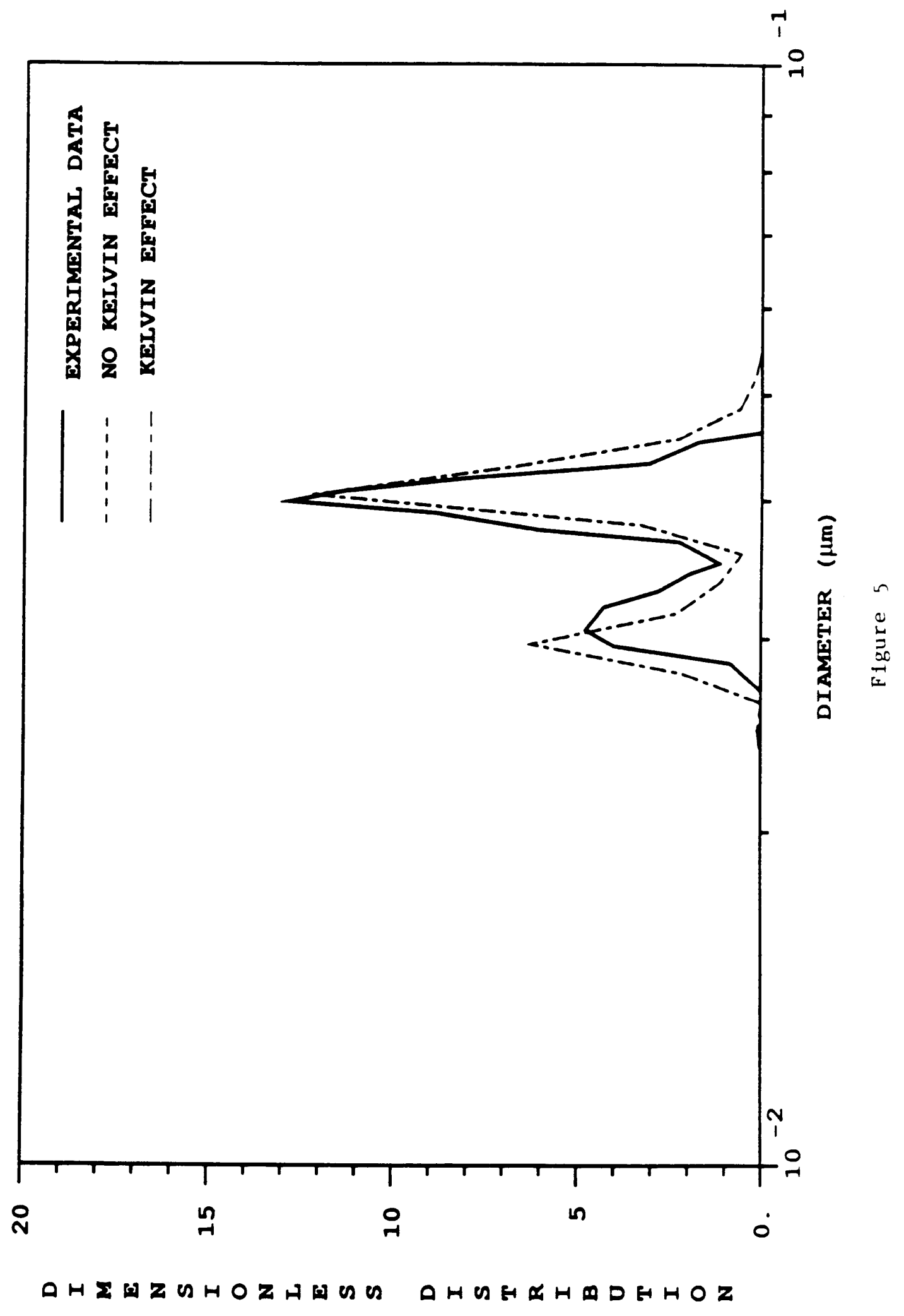




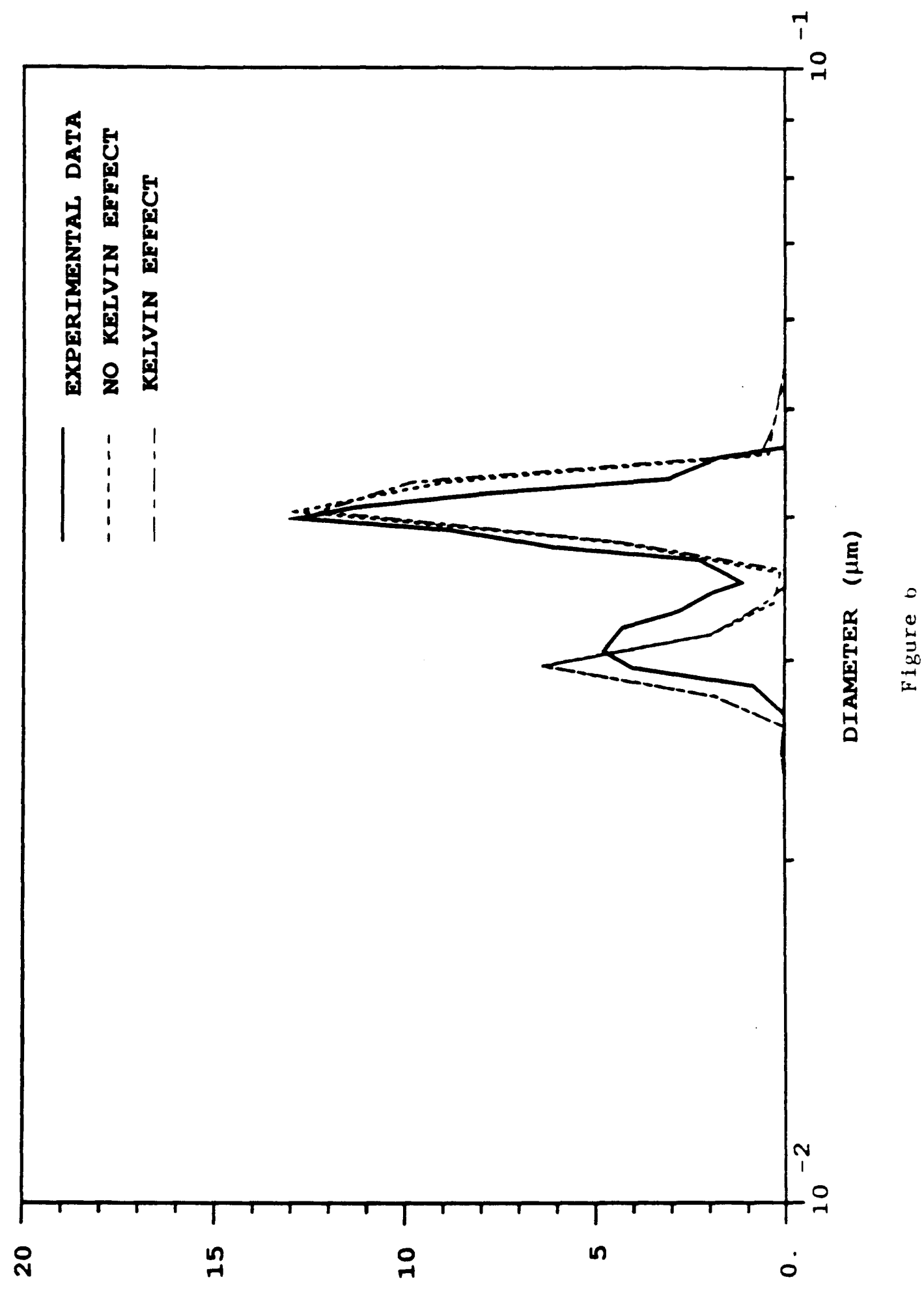

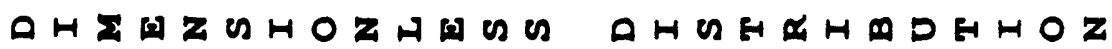


Chapter V

CONCLUSIONS 


\section{SUMMARY}

The concept of mixed particles, particles of the same size but different chemical composition, is not well defined and understood. Recent measurements show that the number-chemical composition distribution is important to understand aerosol properties. Several issues of the distribution are discussed in Chapter I: importance, definition, measurement techniques and data, numerical routines, and further research needs. The sectional representation has been successful in simulating multicomponent aerosol dynamics since it is based on the one-dimensional computational domain, size, though only mean mass fractions of individual species in a section are obtained. A numerical scheme which can simulate mixed particles while having the advantages of the sectional representation, the extended moving sectional method, is presented in Chapter II in which sections move with the characteristics of condensation/evaporation rates. While accurate and efficient, it is only suitable for simulating condensation/evaporation. A numerical method that can simulate mixed particles with simultaneous processes is developed in Chapter III. In this method, contrary to the sectional methods, no assumption is made on the nature of the distribution and a multidimensional hyperbolic partial differential integral equation is directly solved numerically. The routine is applied to simulate the experimental data by Liu et al. (1) and the numerical results agree with the experimental data well.

The numerical methods presented, the extended moving sectional method and the fully multidimensional method, should have a significant impact on the aerosol research area. These can simulate the number-chemical composition distribution that has been a major goal of the aerosol research community. The extended moving sectional method is ideally suited for simulating condensation/evaporation with several unique advantages: (1) it can simulate mixed particles on the one-dimensional computational domain; (2) it can simulate combination of condensation and evaporation; 
and (3) it has no limitation on the :umber of chemical species. The fully multidimensional scheme also has a unique advantage; it can simulate simultaneous processes. These features are a big improvements over other numerical routines.

\section{RECOMMENDATIONS FOR FUTURE RESEARCH}

There are clear areas for improvement in developing numerical routines for simulating aerosol dynamics. First, the required computing time for simulating arbitrarily mixed particles is quite long even considering the current progress in computer development. Thus, ways that can reduce the necessary computing time are neederi. Application of promising numerical schemes is in progress, for example, the meth ods of weighted residuals on coagulation simulation (2) have been tried. Second. it is desirable to incorporate other effects such as thermal (2-5) and equilibrium (6.i) effects between the gas phase and the aerosol phase and the spatial inhomogeneity of aerosols. 


\section{REFERENCES}

(1) Liu, B.Y.H., Pui, D.Y.H., Whitby, K.T., Kittelson, D.B., Kousaka, Y., and McKenzie, R.L. Atmos. Environ. 1987, 12, 99-104.

(2) Wheatley, C.J. CHARM User Manual; NUREG/CR-5162, SAND88-0745, R7, Sandia National Laboratories, 1988.

(3) Gelbard, F. Aerosol Sci. Technol. 1990, 12, 390.112.

(4) Gelbard, F. MGA User Manual; Sandia Nationaı Laboratories, 1990.

(5) Tsang, T.H.; Cook, S.M.; Marra, M.E. Aerosol Sci. Technol. 1990, 12, 386398.

(6) Simons, S. J. Phys. D: Appl. Phys. 1984, 17, 1797-1805.

(7) Park, J.W.; Loyalka, S.K. Nucl. Sci. Eng. 1989, 101, 269-279. 
Appendix I

\title{
NUMERICAL ISSUES IN GRID-BASED PHOTOCHEMICAL AIR QUALITY MODELS
}

\author{
Report to
}

Southern California Edison Company

Rosemead, California

In 1989 


\section{PART A NUMERICAL ASPECTS OF GAS-PHASE PHOTOCHEMICAL AIR QUALITY MODELS}

\section{A.1 Advection}

Because of the infeasibility of simultaneously solving for all three directions in a three-dimensional grid-based photochemical air quality model, an operator splitting algorithm is generally used in which the urizontal and vertical transport steps are computed separately. The two-dimensional horizontal advection operator is the solution of the advection portion of the problem,

$$
\frac{\partial c}{\partial t}=-\frac{\partial(u c)}{\partial x}-\frac{\partial(v c)}{\partial y}
$$

where $c$ denotes the concentration of a species and $u, v$ are the wind velocities in the $x, y$ directions.

Eq. (1) is a first-order hyperbolic partial differential equation, the numerical solution of which has received a great deal of attention (see, for example, [34]). Numerical dispersion and/or diffusion errors are the particular problems associated with the discrete approximation of partial derivatives of Eq. (1). Since these errors play an important role in choosing the best schemes, they require a brief explanation.

For simplicity, consider a one dimensional constant velocity advection system,

$$
\frac{\partial c}{\partial t}=-u \frac{\partial c}{\partial x} .
$$

Let $c_{j}^{n}$ be a discretized value of $c$ at time $t_{n}$ and grid point $x_{j}$ and $T$ denote a specific numerical method that advances the solution from time step $t_{n-1}$ to time step $t_{n}$. Then the relation

$$
c_{j}^{n}=T\left(c_{l}^{n-1}\right) \quad l=1, \ldots, j, \ldots, n s
$$

describes the advance of the numerical solution one time step, where $n s$ is the number of spatial grid points. 
A widely used error analysis technique for numerical methods is the Fourier expansion analysis $[34,40]$. In this approach the numerical solution can be expressed by a finite Fourier series expansion, each Fourier component of which is,

$$
c_{j}^{n}=c^{n}(k) e^{i h j \Delta x}
$$

where $c^{n}(k)$ is the amplitude function at time level $n, i=\sqrt{-1}, k$ is wave number and $\Delta x$ is distance between two grid points. Each Fourier component is a convenient test function to see how the numerical solution for a specific numerical method evolves. The amplification factor for each $k$ is defined as,

$$
A(k)=\frac{c_{j}^{n}}{c_{j}^{n-1}}=\frac{T\left(c_{l}^{n-1}\right)}{c_{j}^{n-1}} .
$$

Usually, $A(k)$ is a complex number and can be written

$$
A(k)=|A(k)| e^{i \phi(k)}
$$

where $|A(k)|$ is the amplitude ratio and $\phi(k)$ is the phase shift of wave number $k$ over one time step.

Since a specific numerical method $T$ gives only an approximate solution of the exact equation, the phase shift of Eq. (6) is generally different from that of the exact solution. This discrepancy between the two phase shifts gives rise to the numerical dispersion errors. If solutions of each wave number propagate at speeds different from those of the exact solution, the result is spurious oscillations around the true solution peak and/or negative values and these phenomena are called numerical dispersion errors. Numerical dispersion errors often cause instability since these errors generally increase with time.

If the amplitude ratio is less than one, the magnitude of the numerical solution is dampened compared to the exact solution. This dissipation induces so-called numerical diffusion errors since the peak in the solution appears to be diffusing away in time. Note that this diffusion error arises from numerical errors, and is not a physical phenomenon. Also note that when the amplitude ratio is larger than one, the numerical solution becomes unstable. This boundary is called the von Neumann stability 
criterion [40]. If the amplitude ratio is exactly one, there are no numerical diffusion errors. Numerical dispersion and diffusion errors are schematically shown in Figure 1.

Numerous numerical schemes have been developed to solve Eq.(1) and their performance has been compared by many researchers. The aim of this section is to perform an objective assessment of the published comparisons of numerical schemes to solve Eq. (1) and to recommend the best schemes for use in grid-based photochemical air quality models.

The abbreviations of some well known numerical methods for the advection equation are given in Table 1. Papers that compare the performance of these methorts are listed in Table 2. Table 3 presents properties of the numerical methods shww in Table 2. Some numerical methods not compared in the papers listed in Table ?. but widely regarded as important schemes, are also given in Table 3. In Tablen ! and 5, quantitative results of comparisons of some numerical methods from Tahle 3 are given. For reference, the advection schemes being used in current grid-based photochemical air quality models are indicated in Table 6 .

The desirable properties of a good numerical advection scheme are in descending priority:

1. Positivity.

2. Accuracy.

3. Mass conservation.

4. Minimum memory size.

5. Fast computing time.

There is a need to comment on the priority list. The advection operator is a part of the full grid-based photochemical air quality model, which includes a gas-phase chemical kinetics operator which will be addressed later. Negative concentrations are not 
allowed. By the same token, mass conservation is an important desirable property. But in some numerical methods, such as a first-order upwind difference method, even though positivity and mass conservation are guaranteed overall accuracy is unacceptably poor. Memory size and speed of computers have increased markedly. Since a two-dimensional space does not require substantial memory size, the minimum memory size required for the calculation of the two-dimensional advection step is not the major criterion for choice of a method. In addition, the computing time required for the advection portion of the overall grid-based air quality model solution is quite small compared to that required for the chemical kinetics. Thus, the computing time required for the advection step is not the major criterion for choice of a method.

An important point to remember is that the above properties of a good advection scheme are not independent but are interrelated. In many cases, to maintain one property one has to sacrifice other properties to some extent. But, at the least. a good advection numerical method should satisfy the first three properties.

Other evaluations of numerical methods have used different criteria and priorities. For example, Chock and Dunker [8] and Chock [7] selected numerical methods based on the same criteria as in this report but put accuracy and computing time as high priorities.

One caution is that the performance of a numerical method is often dependent on the model problem and perhaps other factors like the time integration method and/or the type of computer used. Therefore, one should be aware that a numerical scheme judged best on the basis of one test case may not necessarily be the best method for all cases.

The pseudospectral method has been reported as the most accurate numerical method among those tested by Chock and Dunker [8] and Hov et al. [26]. While Hov et al. [26] recommended the pseudospectral method as the best scheme, Chock and Dunker [8] did not recommend this method because of several limitations such as a small Courant number, a long computing time and a limitation on temporal integration scheme. Also the pseudospectral method requires a periodic boundary 
condition which is difficult to implement in an real atmospheric model. Therefore, the pseudospectral method is not considered further here as a candidate for use in grid-based air quality models.

In the Galerkin method (G-FEM), a kind of finite element method, the numerical solution is approximated by the linear expansion of basis functions. This method requires the residual (error) be orthogonal to the basis functions. The simplest choice of the basis functions is a hatshaped piecewise linear function (chapeau function) [29]. This method has been widely used since it is accurate and its performance is well proven for many cases. G-FEM, however, exhibits difficulties in solving stably a hyperbolic partial differential equation such as Eq. (1) due to the properties of the hyperbolic equation, the propagation of waves, which result in the numerical dispersion errors [29]. Several improvements have been proposed to cope with this difficulty. In asymmetric G-FEM [5, 21], an upwinding term is added to the basis functions to increase the numerical diffusion effects to dampen the dispersion effects. Another approach is to add a numerical filter to remove the numerical dispersion effects [12]. These improvements can reduce the numerical dispersion errors, but at the cost of increasing numerical diffusion errors.

The flux-corrected transport (FCT) method is a technique developed to maintain both the positivity and accuracy of the numerical solution. The idea of the SHASTA method [2, 3], a basic one-dimensional version of FCT, is to transport strongly diffused convective quantities and then to use a nonlinear filter to subtract the diffused amounts from the transported quantities with the constraint of keeping the solution monotone. Zalesak [61] developed a generalized fully multidimensional FCT (G-FCT). These methods have been widely used since they keep the solution positive and reasonably accurate. Also mass is conserved in these methods.

Smolarkiewicz $[43,44]$ developed the repeated upwind difference method (SMO). This method is similar to G-FCT except for the numerical advection scheme and the form of nonlinear filter. A first-order upwind difference method was used as the advection scheme. It has several desirable properties; it is stable and free from 
numerical dispersion errors, conserves total number concentration during condensation/evaporation processes, maintains positivity, is easy to formulate and requires less computing time than higher-order methods. But it produces excessive numerical diffusion errors. The excessively diffused amount is subtracted from the transported quantity and added back to the original grid cell by defining a so-called . idiffusion velocity from a Taylor expansion analysis. The first order upwind difference method is applied repeatedly if necessary. The strengths of this scheme are: (1) It has the desirable properties of the upwind difference methods; (2) It is a fully multidimensional method and can be used without adopting the spatial fractional time steps scheme; (3) It is easy to formulate; and (4) It gives reasonably accurate results with relatively short computing time.

A two-dimensional rotating cosine hill problem has been widely used as a model problem to test numerical advection methods. Comparisons by Chock [7] and Chock and Dunker [8] and Smolarkiewicz [43,44] are shown in Tables 4 and 5, respectively. The finite element method with Forester's method (F-FEM) gives better results (accuracy, computer time and memory) than either the generalized flux-corrected transport method (G-FCT) or the repeated upwind difference method (SMO). F-FEM predicts more accurate peak values than G-FCT and SMO with two iterations. SMO with four iterations gives slightly better results than the F-FEM but requires about two times more computing time. Also F-FEM needs smaller memory than G-FCT. But in F-FEM, one has to solve a matrix system though the matrix is usually sparse and banded while SMO does not require matrix operation.

Yamartino et al. [57] modified a F-FEM method developed by McRae et al. [31] for the CIT air quality model and formulated it for an arbitrary grid system. They reported that for the two-dimensional rotating cosine hill case, the modified F-FEM and the original F-FEM gave almost the same results. Aside from the transport of a point source case, the modified F-FEM performed better than the original one. FFEM occasionally gives negative concentrations when the nonlinear filter of Forester's method does not work well. Forester's method smooths out ripples of a certain 
wavelength. To maintain the positivity, one has to smooth out a broader range of ripples and the peak values are also smeared out as shown in Table 4. The G-FCT and SMO give similarly good results and guarantee positivity. For the same accuracy the G-FCT requires more computing time than SMO.

Based on the above criteria and considerations, we recommend the finite element method with Forester's method and the Smolarkiewicz's repeated upwind difference method as the best numerical advection schemes for grid-based photochemical air quality models. Both methods can be implemented easily. Quite interestingly, these two methods are currently used in grid-based air models, F-FEM in the CIT model, the modified F-FEM in the State of California Air Resources Board model (CALGRID) and SMO in the Systems Applications Inc. UAM. Thus, further effort on the development of methods for the advection portion of photochemical models does not seem warranted at this time.

\section{A.2 Chemical Kinetics}

The chemical kinetics operator is the solution of the gas-phase chemical rate reaction equations (ODEs),

$$
\frac{d c_{i}}{d t}=f_{i}\left(c_{1}, \ldots, c_{s}, t\right) \quad i=1, \ldots, s
$$

with an appropriate set of initial conditions, where $c_{i}$ denotes the concentration of a species $i, s$ is the number of components, and $f_{i}$ is the temperature dependent chemical reaction rate of species $i$.

The difficulties in solving Eq. (7) for atmospheric mechanisms are well documented [31]. One difficulty arises from the nonlinearity of the kinetic forms; another is a result of the fact that the number of species and the number of reactions are usually large and the reaction rates must be calculated at every grid point. The most serious difficulty, however, arises because the characteristic time scales of the reactions differ by orders of magnitude, i.e., the chemical kinetic system is stiff $[9,31]$. Eq. (7) is called stiff if 
1. $\operatorname{Re}\left(\lambda_{i}\right)>0 \quad i=1, \ldots, s$.

2. $\left(\max \left|\operatorname{Re}\left(\lambda_{i}\right)\right|\right) /\left(\min \left|\operatorname{Re}\left(\lambda_{i}\right)\right|\right)=\mathrm{R} \gg 1 \quad i=1, \ldots, s[31]$,

where $\mathrm{R}$ is the so-called stiffness ratio and $\lambda_{i}$ are the eigenvalues of the Jacobian matrix $\bar{J}=\frac{\partial \bar{f}}{\partial \bar{c}}$, where upper bars denote vectors. The concept of stiffness can be also explained physically. If we rewrite Eq. (7) as

$$
\begin{aligned}
\frac{d c_{i}}{d t} & =Q_{i}(t)-\frac{c_{i}(t)}{\tau_{i}(t)} \\
& =f_{i}\left(c_{1}, \ldots, c_{s}, t\right) \quad i=1, \ldots, s
\end{aligned}
$$

where $Q_{i}(t)$ is the generation rate and $\frac{c_{i}(t)}{\tau_{i}(t)}$ is the loss rate of species $i, \tau_{i}(t)$ is $t_{1}$. characteristic relaxation time describing how quickly species $i$ reaches its equilibrinu value. If the values of $\tau_{i}(t), i=1, \ldots, s$ vary by many orders of magnitude. the system is called stiff. In other words, some species reach their equilibrium value, very quickly while others reach their equilibrium values very slowly. The difficult! with stiff problems is that for conventional numerical methods, the time step size is determined by the minimum $\tau_{i}(t)$ due to consideration of stability, while the total simulation time is governed by the maximum $\tau_{i}(t)$. Thus, if the small step sizes dictated by stability are used, the computing time is prohibitively long. Due to the considerable spread in time scales of the species, the calculation of chemical kinetics in a photochemical air quality model requires the major portion of the computing time.

Many methods have been developed to solve stiff ODEs. The aim of this section is to assess those published stiff ODEs solvers to solve Eq. (7) and to recommend the best schemes for use in grid-based photochemical models. In Table 7, several numerical methods for stiff ODEs are listed. Particular integration packages that can handle stiff ODEs and the numerical methods on which they are based are given in Table 8. For reference, the stiff ODEs solvers used in current grid-based photochemical air quality models are summarized in Table 9. 
The desirable properties of a good numerical stiff ODEs solver are in descending order:

1. Computing time.

2. Memory size.

\section{Accuracy.}

Since the chemical kinetics calculation consumes the predominant part of the air quality model computing time, a stiff ODE solver with short computing time has a significant advantage over other solvers with longer computing time even at the expense of some accuracy. Since the chemical kinetics has to be calculated at every three-dimensional grid point, the memory size requirement is also important. In that sense, a single step method is preferable to a multistep method since only one previous time level of information is stored. With respect to accuracy, as pointed out by Young and Boris [60], there is no need that the errors in the calculation of chemical kinetics should be significantly less than those from the calculation of other processes.

Integration packages such as GEAR [23], EPISODE [25] and LSODE [24], which use the backward differentiation formulae (BDF), have been tested extensively and are regarded as some of the most accurate and rigorous methods for stiff ODEs. But the BDF are implicit multistep methods, in which information from several previous time steps must be stored for later use. Also in implicit methods, a nonlinear alg. raic system must be solved which requires matrix operations, although sometimes one can take advantage of the fact that the matrix is sparse and/or banded. Implicit or semiimplicit Runge-Kutta methods have been widely tested and well proven, but these methods again require matrix operations and long computing times [18, 34].

LARKIN [11] uses a semi-implicit midpoint rule, a kind of extrapolation method, and has shown results comparable to GEAR. Deufhand et al. [11] compared GEAR, a BDF solver which is not effective at solving very stiff ODEs [25], GRKF4, a semi-implicit Runge-Kutta method and METAS1, the main stiff ODEs suver in the 
LARKIN on several examples. They reported that METAS1 was comparable to GEAR in computing at the same accuracies, while GRKF4 spent a longer computing time than two other methods at the same accuracies. These methods, either multistep or/and implicit methods, have severe disadvantages of long computing time and large memory size.

In CHEMEQ [59, 60], an asymptotic integration method [34] is used to solve the stiff ODEs and a normal second-order accurate predictor-corrector method is used for the nonstiff ODEs. The asymptotic method best treats the stiff problem when the solution is slowly changing, but the time constants are prohibitively small. This occurs when both the generation rate $\left(Q_{i}\right)$ and loss rate $\left(c_{i} / \tau_{i}\right)$ are large but nearly. equal, and there is strong coupling among the equations. This method is stable and tends to damp out small oscillations caused by very small time constants. The secondorder predictor-corrector method is employed to continue the integration process. $A$ crucial part of the method is to identify the stiff equations and to determine the initial time stepsize. The initial time stepsize is determined by

$$
\Delta t=\epsilon \min \left[\frac{c_{i}}{f_{i}}\right]
$$

or if $Q_{i}>>c_{i} / \tau_{i}$, then

$$
\Delta t=\epsilon \min \left[\tau_{i}\right]
$$

where $\epsilon$ is. a scale factor, typically the same as the convergence criterion that will be discussed shortly. If the stepsize is greater than a specified value, the equation is considered stiff throughout the integration process and is integrated according to the asymptotic method.

The predictor part of the step is

$$
\begin{array}{ll}
c_{i}(1)=c_{i}(0)+\Delta t f_{i}(0) & \text { (normal) } \\
c_{i}(1)=c_{i}(0)+\frac{c_{i}(0)\left[2 \tau_{i}(0)-\Delta t\right]+2 \Delta t \tau_{i}(0) Q_{i}(0)}{2 \tau_{i}(0)+\Delta t} & \text { (stiff) }
\end{array}
$$

where $f_{i}(0)=f_{i}\left[t(0), c_{i}(0)\right]$ and $c_{i}(k)$ is the $k$-th iterated value of $c_{i}$, or an approximation to $c_{i}(t(0)+\Delta t)$. Also $f_{i}(k)=f_{i}\left[t(0)+\Delta t, c_{i}(k)\right]$. The correction part of the 
step is

$$
\begin{array}{rlr}
c_{i}(k+1)= & c_{i}(0)+\frac{\Delta t}{2}\left[f_{i}(0)+f_{i}(k)\right] & \text { (normal) } \\
c_{i}(k+1)= & c_{i}(0) & \text { (stiff) } \\
& +\frac{c_{i}(0)\left[\tau_{i}(0)+\tau_{i}(k)-\Delta t\right]+\frac{\Delta t}{2}\left[\tau_{i}(0)+\tau_{i}(k)\right]\left[Q_{i}(0)+Q_{i}(k)\right]}{\tau_{i}(0)+\tau_{i}(k)+\Delta t} .
\end{array}
$$

By comparing $c_{i}(k+1)$ with $c_{i}(k)$ for all of the $s$ equations using the relative error criterion,

$$
\max \left[\frac{\left|c_{i}(k+1)-c_{i}(k)\right|}{c_{i}(k+1)}\right] \leq \epsilon,
$$

convergence of each of the individual equations can be determined. A fixed, small number of iterations are carried out each step, and whether or not convergence is achieved determines how the step size will change in the next time step. The method is stable, efficient, and generally accurate. Since it is self-starting and is a one-step method, it requires small memory size. Also it does not require matrix operations. One problem of the method is that it is not inherently conservative. Conservation is controlled by adjusting the convergence criterion.

Young and Boris [60] compared several numerical schemes, GEAR, a second order predictor-corrector method, KREGEL (an implicit linear multistep predictorcorrector method [34]) and CHEMEQ. They reported that both CHEMEQ and KREGEL had about the same order of accuracy (second order) and CHEMEQ spent less computing time than others for the same accuracies. Also McRae et al. [31] compared EPISODE and CHEMEQ. They reported that the maximum relative error after 120 minutes simulation was about six percent (using EPISODE as a reference) and CHEMEQ required only one-third the computing time of EPISODE.

CHEMEQ satisfies most of the criteria discussed earlier. Contrary to other multistep and/or implicit methods mentioned above, BDF, (semi-)implicit Runge-Kutta methods and (semi-)implicit extrapolation methods, the method requires information from only the previous time step and does not require matrix operations. Thus, computing time and memory size problems are reduced to manageable sizes. Further- 
more, this method is reasonably accurate as compared above. Therefore, CHEMEQ is favorable to other methods mentioned above.

CREK1D, developed by Pratt and Radhakrishnan [37], uses an exponentially fitted trapezoidal method. They reported that the CREK1D has a polynomial order accuracy of at least two, but the general performance of the exponential fitting methods is similar to that of BDF [34]. Therefore, there is no merit to use an exponentially fitted extrapolation method over the asymptotic integration method.

Carmichael et al. [6] used a semi-implicit Euler method developed by Preussener and Brand [38]. This method is a one-step method and fast but its accuracy is of first order which is usually lower than those of the numerical methods for other processes.

In a quasi-steady state approximation (QSSA) method [22], a kind of locallyexact solution method, all coefficients of the ODEs in one time step are assumed to be constant and the exact solutions of the approximate equations are calculated for some species determined before each integration step. The criteria and the integration scheme are as follows: (1) If $\tau_{i}<\Delta t / 10$,

$$
c_{i}(t+\Delta t)=Q_{i}(t+\Delta t) \tau_{i}(t+\Delta t) .
$$

(2) If $\Delta t / 10 \leq \tau_{i} \leq 100 \Delta t$,

$$
c_{i}(t+\Delta t)=c_{i e}+\left[c_{i}(t)-c_{i e}\right] \exp \left[-\frac{\Delta t}{\tau_{i}}\right],
$$

where $c_{i e}=Q_{i} \tau_{i}$ and assumed as a constant. If $\tau_{i}>\Delta t$,

$$
c_{i}(t+\Delta t)=c_{i}(t)+\left[Q_{i}(t)-\frac{c_{i}(t)}{\tau_{i}(t)}\right] \Delta t .
$$

Yamartino et al. [57] tested Gear's method, the asymptotic method, and the QSSA method for some model problems. They reported that all three methods gave similar results while the computing time ratio was 10:1.5:1 in order of Gear's method, the asymptotic method, and the QSSA method. While fast and accurate in some cases, the QSSA method has a severe time step limitation and a problem of choosing a appropriate time step since the QSSA is usually valid only with a very small time 
step. Also this method is basically of first-order accuracy since it uses the Euler method for species with longer photochemical lifetimes. As mentioned by the authors [22], this method is views as an alternate method.

The Urban Airshed Model (UAM) developed by Systems Applications, Inc. uses Crank-Nicholson method to convert the kinetics ODEs to nonlinear algebraic equations and uses Newton's iteration method to solve the algebraic equations [39]. While stable, it is a conventional implicit finite difference method and suffers from numerical errors, especially numerical dispersion errors [34]. Also it is less suitable for stiff ODEs than several of the other methods cited above. In the CIT model, some species are approximated to be in pseudo steady state (PSSA) to reduce the computing burdens [31]. The chemical kinetics of other species are simulated by CHEMEQ. In the CALGRID, the PSSA is also applied and both QSSA and CHEMEQ are installent and can be chosen by the users to do temporal integration of chemical kinetics.

Based on the above comparisons, we recommend for grid-based air quality morlets CHEMEQ, a hybrid predictor-corrector method of the asymptotic integration form. coupled with the Euler method.

\section{A.3 Summary of Numerical Issues in}

Gas-Phase Photochemical Air Quality Models

Our analysis of the numerical elements of current gas-phase photochemical air quality models indicates that the numerical techniques have generally been optimized for the particular structure of these models. Therefore, we do not recommend any further projects aimed at fine-tuning the methods that are currently incorporated (see Table 10 for the methods). On the other hand, with the advent of massively parallel computer architecture, one could envision eventually re-programming these models for concurrent computers. This could be a major undertaking, with the possible benefit of greatly decreased computer execution time. 


\section{PART B. NUMERICAL ASPECTS OF}

\section{ATMOSPHERIC AEROSOL MODELS}

In the second half of this report we turn our attention to numerical issues in aerosol and $\mathrm{PM}_{10}$ models. Although such models, at least from the point of view of atmospheric applications, are less well developed than those for gas-phase species, the importance of $\mathrm{PM}_{10}$ modeling dictates that such models will assume an ever expanding role in the near future. We begin with a consideration of the aerosol general dynamic equation which is, in principle, the basis for a $\mathrm{PM}_{10}$ model. We first discuss the numerical solution of the general dynamic equation and then proceed to episodic $\mathrm{PM}_{10}$ models, which have their special requirements relative to numerical aspects.

\section{B.1 The General System}

In its most general form, the multicomponent general dynamic equation (GDE) governing the size-composition distribution function of an aerosol, $n(m, t)$, is (spatial dependence is omitted)

$$
\begin{aligned}
& \frac{\partial n(m, t)}{\partial t}+\sum_{i=1}^{s} \frac{\partial}{\partial m_{i}}\left[I_{i}(m, t) n(m, t)\right] \\
& =\frac{1}{2} \int_{0}^{m_{1}} \cdots \int_{0}^{m_{s}} \beta(u, m-u) n(m-u, t) n(u, t) d u \\
& -n(m, t) \int_{0}^{\infty} \cdots \int_{0}^{\infty} \beta(u, m) n(u, t) d u
\end{aligned}
$$

where $m_{i}$ is the mass of the $i$-th component in a particle, and $m$ is a vector of compositions $\left(m_{1}, \ldots, m_{s}\right)$, where $s$ is the total number of components. $n(m, t) d m$ is the number of particles having mass of component $i$, in the range $\left[m_{i}, m_{i}+d m_{i}\right]$ at time $t$. $I_{i}$ is the time race change of the mass concentration of the $i$-th component from condensation/evaporation processes. $\beta(u, m)=\beta(m, u)$ is the binary coagulation coefficient. The initial condition is

$$
n(m, 0)=n_{o}(m)
$$


The boundary condition specifies no particles of zero size,

$$
n(0, t)=0
$$

The multicomponent aerosol GDE presents several computational challenges because of the inherently different nature of condensation/evaporation and coagulation processes, the wide range of magnitudes of the dependent and independent variables, and because of the multidimensional nature of the size-composition distribution function. Condensation/evaporation are number conserving processes, while coagulation is a mass conserving process. The characteristic times of the processes in Eq. (17) may differ by several orders of magnitude. Numerical methods that conserve the total number concentration during condensation/evaporation (for evaporation number conservation may be applied to particles larger than the critical size) and conserve the total mass concentration during coagulation are preferred. To simulate processes not explicitly indicated in Eq. (17), such as deposition or nucleation, is not difficult once coagulation and condensation/evaporation are adequately handled.

Considerable effort has been devoted to the development of numerical techniques for solving the aerosol GDE [28, 42]. These efforts can be classified according to whether they address single component or multicomponent aerosols, which processes. notably coagulation and/or condensation/evaporation, are occurring, and whether the methods have been applied to real or hypothetical situations. Table 11 summarizes many of the previous approaches organized by these criteria.

From Table 11, following observations can be made:

- Single component aerosol systems have been mainly treated in the literature.

- The only numerical method currently available for multicomponent systems is the sectional method.

- In many cases, hypothetical situations have been treated in the literature and the numerical methods used in those studies are not generally 
applicable to an episodic $\mathrm{PM}_{10}$ model.

\section{B.2 Numerical Solution of the Single Component}

Aerosol General Dynamic Equation

Simulation of the single component aerosol GDE has been an interesting and important topic since the early 1970s and several numerical schemes have been developed. Seigneur et al. [42] compared several of those schemes for the simulation of atmospheric aerosol dynamics. Simulation of single component aerosol dynamics with a single process occurring (condensation and evaporation are considered as the same process since both processes are described by the same mathematical form) is easier than the simulation of simultaneous processes because of the conflicting demands imposed by condensation/evaporation versus coagulation. In this section, three cases are discussed; pure coagulation, pure condensation/evaporation, and simultaneous condensation/evaporation and coagulation.

The so-called moment methods are aimed at obtaining moments of the size distribution, such as the total number, the total mass (or volume), or the total surface area concentration by assuming either the form of the size distribution $[4,42]$ or other properties to describe the evolution of the aerosol size distribution. Though these moment methods are relatively easy to formulate and need short computing time, the accuracy of these methods is generally poor and thus their use is restricted to special cases and/or crude estimates and will not be considered further here.

To represent the coagulation integrals in Eq. (17) is a demanding problem even in the single component case. Since a logarithmic transformation is usually used to reduce the range of independent variables in Eq. (17), a problem immediately arises because the transformed value of $(m-u)$ in the first term on the right hand side of Eq. (17) does not coincide with the value at the grid points even though both the transformed values of $m$ and $u$ are at grid points. Thus, an interpolation scheme 
must be used in the evaluation of the coagulation integrals.

Gelbard et al. [15] developed the sectional method to treat coagulation. The essential idea of the sectional method is to divide the aerosol size spectrum into a number of sections and assume that the total particle mass size distribution is uniform in each section. The resultant quantities obtained are moments, usually the number or the mass concentration, of each section. Thus this method can be regarded as a moment method on finite elements. The sectional method uses a kind of bookkeeping technique to account for inflows and outflows of particles in each section and transfer between sections to conserve the total mass concentration. The sectional method requires evaluation of the double integrals to calculate the sectional coagulation coefficients and this integration takes the largest portion of the computing time. With the use of the so-called geometric constraint $v_{l} \geq 2 v_{l-1}$, where $v_{l}$ is the upper and $v_{l-1}$ is the lower bound of the particle volume of the $l$-th section, the integration becomes simpler and requires less computing time than without this constraint. The sectional method has been extended to the multicomponent case as will be discussed later.

Brock and coworkers $[32,46]$ used the cubic spline method to calculate coagulation dynamics. The method is rigorous and accurate but is difficult to use it in the multicomponent case due to the excessive computing time.

Seigneur et al. [42] applied several numerical schemes for coagulation to a number of examples of atmospheric aerosol dynamics and compared the results. They observed that COAGUL, a program based on the cubic spline method [46], and ESMAP, a program based on the sectional method [14], gave similar results but that ESMAP needed more computing time for a comparable level of accuracy. Therefore, for single component aerosol dynamics involving coagulation, a numerical scheme based on the cubic spline method [46] is preferred.

The evolution of an aerosol size distribution as a result of condensation/evaporation processes is described by a first-order hyperbolic partial differential equation which also describes advection processes. Numerical difficulties in solving this equation 
accurately and efficiently are described above, especially the problems of numerical dispersion and diffusion. Conventional higher-order accuracy numerical advection methods, such as the Galerkin finite element method, give accurate solutions and have small numerical diffusion errors. Yet these methods usually suffer from numerical dispersion [29]. On the contrary, lower-order accuracy numerical advection methods, such as the first-order upwind difference method, are free from numerical dispersion, but suffer from excessive numerical diffusion.

In general, in numerical methods that calculate the transport of conserved quantities at fixed grids, i.e., using the Eulerian approach, numerical errors arising from the discrete approximation of first-order spatial derivatives (advection terms) cannot l,e eliminated, only reduced. In Lagrangian methods, grids and computational cells mos.. with the conserved quantities along the characteristics thus eliminating numerical in rors due to the discrete approximation of spatial derivatives. The most significant difficulty of applying a Lagrangian approach to aerosol condensation/ evaporation in the distortion of the grids and cells that occurs as particles of different sizes grow or shrink at different rates. Some numerical schemes remap the functional values from the distorted Lagrangian grids onto the regular Eulerian grids by interpolation at each time step. During interpolation, however, new numerical errors are introduced.

Tsang and Brock $[49,50]$ simulated condensation/evaporation by using the finite element method with characteristics [53] and then remapping to a fixed grid b! ubic spline interpolation. This is a kind of Lagrangian-Eulerian approach [33] and can be quite accurate. It can, in principle, be extended to the multicomponent case. Seigneur et al. [42] applied several schemes for condensation. CONFEMM, a program based on the finite element method with characteristics [53], gave globally accurate results though, in some cases, the scheme did not conserve the total number concentration. ESMAP, a program which uses the sectional method developed by Warren and Seinfeld [55] to simulate condensation/evaporation, conserved the total number concentration, but suffered from numerical diffusion errors.

We conclude that for single component aerosol dynamics with condensation/evaporation, 
a numerical scheme based on the finite element method with characteristics $[49,50]$ is preferred.

Simulation of simultaneous condensation/evaporation and coagulation is more difficult than that of either single process. Ideally, both processes should be treated by a single numerical method. But, in practice, the method of fractional steps $[34,58]$ is usually used. In this approach, each process is treated by a different numerical scheme and then the individual results are combined under certain constraints. Fractionation errors are introduced, but this kind of error is an inevitable price one has to pay to solve the simultaneous processes. A more severe limitation arises if the characteristic times of each process differs by several orders of magnitude, and the temporal step size must be limited by the process of which the characteristic time is the shortest.

Tsang and Hippe [51] used the cubic spline method [46] and the finite element method with characteristics [49] with the time splitting method [58] for the case of simultaneous condensation/evaporation and coagulation. The results were accurate but required relatively long computing time. Warren and Seinfeld [55] developed the code ESMAP, based on the sectional method $[15,55]$. This method does not require the time splitting method, but as mentioned earlier, the numerical scheme for condensation/evaporation is only first-order accurate and, as a result, it suffers from numerical diffusion errors.

We conclude that for single component aerosol dynamics with simultaneous condensation/evaporation and coagulation, the approach of Tsang and Hippe [51] is recommended.

\section{B.3 Numerical Solution of the Multicomponent}

Aerosol General Dynamic Equation

Multicomponent aerosol dynamics, a multidimensional problem from the mathematical point of view, presents serious numerical challenges. Simulation of multicomponent aerosol dynamics will be constrained by the computer memory size and computing time for even a modest number of components. For example, calcula- 
tion of multicomponent condensation/evaporation processes can require a substantial computer memory and a long computing time [28]. For an $s$ component system, a finite difference method with $n$ grids for each component needs, for example, $n^{s}$ grid points and a finite element method with $n$ cells for each component requires an $n^{s} \times n^{s}$ matrix system although the matrix is usually banded and/or sparse. Also one has to calculate $s$ condensation rate equations at each grid point $\left(s \times n^{s}\right.$ calculations for a finite difference method). By its nature, the coagulation process also requires lengthy computing times. For an $s$ component system with $n$ grids for each component, using a $q$-point product type Gaussian quadrature and linear interpolation, $\left(n^{s} \times q^{s}+q^{s}\right)$ point calculations and $\left(2 \times s \times n^{s} \times q^{s}+s \times q^{s}\right)$ interpolations are required for each time step.

The method of fractional steps (or time splitting method) [58] has been used in some cases, such as in grid-based air quality models [31], to circumvent the dimensionality problem. In this approach as applied to aerosol dynamics, a multicomponent condensation/evaporation problem is divided into several single component condensation/evaporation problems, each solved respectively, and then combined under some restrictions. While requiring less memory than attacking the full problem directly, errors due to the fractionation are introduced. Thus, if there were a numerical method to solve multicomponent dynamics directly, this direct scheme is preferable to those based on the method of fractional steps.

Relatively few numerical solutions of the multicomponent aerosol GDE exist as shown is Table 11. Presently the only numerical method available for simulating multicomponent aerosol dynamics is the sectional method [14]. In the multicomponent form of the sectional method, in addition to the single component sectional method assumptions, the mean mass fraction of all particles in a section are assumed to be identical. Bassett et al. [1] and Pilinis et al. [35] have applied the multicomponent sectional method to atmospheric aerosol simulations.

In the original fixed grid sectional method [14], the total mass concentration is conserved as in the single component sectional method. Thus the sectional method 
with fixed grids is most suitable for the simulation of coagulation. In the fixed grid sectional method, condensation/evaporation processes are simulated by the method developed by Warren and Seinfeld [55]. As explained in the previous section, while this scheme conserves the total number concentration, it suffers from numerical diffusion errors.

Gelbard $[16,17]$ combined the sectional approach with the Lagrangian concept of a movable grid and developed a moving sectional method for simulating aerosol condensation/evaporation of a single condensable species. In that method the boundaries of each particle size section move with time in accordance with the growth or shrinkage of particles at those boundaries. In that way numerical diffusion errors resulting from the need to apportion the particle size distribution over a set of fixed sections are avoided. Also, numerical dispersion errors resulting from the convection term are avoided since the convection term is automatically handled by solving the characteristic equations. Kim and Seinfeld [28] extended the concept of the moving sectional method to treat multiple condensable species. In the moving sectional method, the deformation associated with the Lagrangian approach provides the advantage that the moving sectional method can simulate particles of the same total mass but different compositions. Since compositions of adjacent sections are discontinuous at the section boundary, condensation/evaporation rates of each section. the boundary are different. As a result, sections can in principle overlap each other or actual vacancies in the calculation domain may develop between sections. To treat coagulation with the moving sectional method is difficult due to the computational domain deformations, overlapping and possible vacancies.

While the assumption that the size distribution in a section is uniform itself introduces numerical diffusion errors, such errors exist even in higher order approximations, so this assumption is best viewed as a necessary mathematical approximation. The use of the same mean mass fraction in a section composition, however, imposes a possibly undesirable physical constraint, namely that particles of the same size should have same mean mass compositions (so-called internally mixed particles) since the 
result of the sectional method is not the size distribution but the moment of the size distribution.

To alleviate the above limitations of the sectional method, a new technique has been developed to numerically solve the multicomponent aerosol GDE directly without introducing any physical assumptions about the nature of the size-composition distribution [27]. The advantages of a direct numerical solution over the sectional method are: (1) The direct solution can simulate arbitrarily mixed multicomponent particles, while the sectional method simulates only internally mixed particles; and (2) The technique gives the size distribution directly, while the sectional method gives moments of the size distribution, integrated quantities over the section. The penalty one pays for these advantages of the direct numerical solution is, of course, in increased computing time and memory size.

The direct solution technique uses the repeated upwind difference method [43, 44] for the simulation of condensation/evaporation and the product type Gaussian quadrature with linear interpolation for the simulation of coagulation, and a time splitting method to combine both methods. The Euler method is used to integrate the coagulation process in time. In a comparison with the SHASTA method, a type of flux-corrected transport (FTC) method [3] that has been widely tested and used, for the simulation of single component aerosol dynamics with simultaneous coagulation and condensation, the repeated upwinding method has been found to be both more accurate and more computationally efficient than the SHASTA method.

Gaussian quadrature with a product rule is selected to perform the multiple integrals. The Gaussian quadrature has been well proved and extensively tested $[10,45]$, and this method is already used in the multicomponent sectional method to evaluate double integrals [54]. As a compromise between the accuracy and the computing burden, linear interpolation is used. Since the interpolation scheme is linear, a higherorder time integration method is unnecessary.

Numerical solutions have been compared to analytical solutions for model problems of pure condensation/evaporation, pure coagulation, and simultaneous conden- 
sation/evaporation and coagulation and good agreement has been obtained. That this direct solution method can simulate, for the first time, particles of same size but different composition is the most important advantage of the direct solution method over the sectional method. Another important difference between the direct solution method and the sectional method is that the latter calculates moments, usually the mass moment, while the direct method calculates the size distribution itself. The advantages of this direct solution are not obtained without a price, namely the computing time of the direct method is considerably longer than that of the sectional method. The ratios of computing time of the direct solution method to that of the sectional method for a three component system are 500 for pure condensation/evaporation. 2202 for pure coagulation, and 1979 for simultaneous processes.

A final note is that the numerical schemes for multicomponent aerosol dynamic are also applicable to the single component aerosol dynamics. For example. the. moving sectional method $[16,17,28]$ is directly applicable to the single componen: case and give very accurate results efficiently.

\section{B.4 Numerical Issues in Episodic $\mathrm{PM}_{10}$ Models.}

A $\mathrm{PM}_{10}$ model refers to an air quality model capable of predicting the quantity and chemical composition of airborne particles of diameters less than $10 \mu \mathrm{m}$. While such a model may have additional size resolution beyond simply one size class from zero to $10 \mu \mathrm{m}$ diameter, it is generally recognized that size distribution is less critical than chemical composition discrimination, from the point of view of air quality maintenance planning, for example, nitrate, ammonium, sulfate, carboneous material, etc. Any $\mathrm{PM}_{10}$ model is based in principle on the aerosol general dynamic equation, but if size resolution is to be integrated out, the model variable will be just the mass concentrations, in $\mu \mathrm{gm}^{-3}$, of each of the components of interest. Coagulation processes then no longer play a role in the model and the principal physical processes that are included are gas-to-particle conversion by condensation/evaporation, together with transport, diffusion and dry deposition. As a result, many of the numerical issues 
discussed above relating to solving the aerosol GDE are not immediately relevant to such episodic $\mathrm{PM}_{10}$ models. On the other hand, it is anticipated that future operational $\mathrm{PM}_{10}$ models will incorporate both size and composition resolution (the model of Pilinis and Seinfeld [36] does so already), and therefore the numerical issues addressed earlier will be highly relevant. 


\section{References}

[1] Bassett, M., Gelbard, F. and Seinfeld, J.H., Atmos. Environ. 15, 2395(1981).

[2] Book, D.L., Boris, J.P. and Hain, K., J. Comp. Phys. 18, 248(1975).

[3] Boris, J.P. and Book, D.L., J. Comp. Phys. 11, 38(1973).

[4] Brock, J.R. and Oates,J., J. Aerosol Sci. 1, 59(1987).

[5] Brooks, A.N. and Hughes, T.J.R., J. Comp. Methods in Appl. Mech. Eng. 32, 199(1982).

[6] Carmichael, G.R., Peters, L.K. and Kitade, T., Atmos. Environ. 20, 173(1986).

[7] Chock, D.P., Atmos. Environ. 19, 571(1985).

[8] Chock, D.P. and Dunker, A.M., Atmos. Environ. 17, 11(1983).

[9] Curtiss, C.F. and Hirschfelder, J.O., Proc. Natl. Acad. Sci. 38, 235(1952).

[10] Davis, P.J. and Rabinowitz, R., Methods of Numerical Integration, Academic Press, New York(1975).

[11] Deufhand, P., Bader, G. and Nowak, J., in Modelling of Chemical Reaction Systems, ed. Ebert, K.H., Deufhand, P. and Jaeger, W., SpringerVerlag, Berlin(1981).

[12] Forester, C.K., J. Comp. Phys. 23, 1(1977).

[13] Gelbard, F. and Seinfeld, J.H., J. Comp. Phys. 28, 357(1978).

[14] Gelbard, F. and Seinfeld, J.H., J. Colloid Interface Sci. 78, 485(1980). 
[15] Gelbard, F., Tambour, Y. and Seinfeld, J.H., J. Colloid Interface Sci. 76, 541(1980).

[16] Gelbard, F., in "Proceedings of American Association for Aerosol Research" Seattle, (1987).

[17] Gelbard, F., "Modeling Multicomponent Aerosol Particle Growth by Vapor Condensation," Aerosol Sci. Technol. (in press).

[18] Hairer, E., Norsett, S.P. and Wanner, G., Solving Ordinary Differential Equations I Nonstiff Problems, Springer-Verlag, Berlin(1987).

[19] Harten, A., J. Comp. Phys. 49, 357(1983).

[20] Harten, A. and Osher, S., SIAM J. Numer. Anal. 24, 279(1987).

[21] Heinrich, J.C., Huyakorn, P.S., Zienkiewicz, O.C. and Mitchell, A.R., Int. J. Numer. Meth. Eng. 11, 131(1977).

[22] Hesstvedt, E., Hov, $\emptyset$. and Isaksen, I.S.A., Int. J. Chem. Kinet. X, 971(1978).

[23] Hindmarsh., A.C., Lawrence Livermore Laboratory Report UCID30001, Rev. 3 (1982).

[24] Hindmarsh., A.C., Lawrence Livermore Laboratory Report UCRL88007 (1982).

[25] Hindmarsh., A.C. and Byrne, G.D., Lawrence Livermore Laboratory Report UCID-30112, Rev. 1 (1977).

[26] Hov, Ø., Zaltev, Z., Berkowicz, R., Eliassen, A. and Prahm, L.P., Atmos. Environ. 23, 967(1989).

[27] Kim, Y.P. and Seinfeld, J.H., J. Aerosol Sci. submitted (1990). 
[28] Kim, Y.P. and Seinfeld, J.H., J. Colloid Interface Sci. in press, (1990).

[29] Lapidus, L. and Pinder, G.F., Numerical Solution of Partial Differential Equations in Science and Engineering, John Wiley \& Sons, New York(1982).

[30] Long,Jr., P.F. and Pepper, D.W., J. Appl. Met. 20, 146(1981).

[31] McRae, G.J., Goodin, W.R. and Seinfeld, J.H., J. Comp. Phys. 45, $1(1982)$.

[32] Middleton, P. and Brock, J., J. Colloid Interface Sci. 54, 249(1976).

[33] Neuman, S.P., J. Comp. Phys. 41, 270(1981).

[34] Oran, E.S. and Boris, J.P., Numerical Simulation of Reactive Flow, Elsevier, New York(1987).

[35] Pilinis, C., Seinfeld, J.H. and Seigneur, C., Atmos. Environ. 21, 943(1987).

[36] Pilinis, C., Seinfeld, J.H. and Seigneur, C., Atmos. Environ. 22, 1985(1988).

[37] Pratt., D.T. and Radhakrishnan, K., NASA Technical Memorandum 83806 (1984).

[38] Preussener., P.R. and Brand, K.P., Chem. Eng. Sci. 36, 1633(1981).

[39] Reynolds, S.D., Ames, J., Hecht, T.A., Meyer, J.P., and Whitney, D.C., EPA-600/4-76-016B, (1976).

[40] Roache, P.J., Computational Fluid Dynamics, Hermosa Publishers, Albuquerque(1972).

[41] Russell, A.G. and Cass, G.R. (1984) Atmos. Environ. 18, 1815. 
[42] Seigneur, C., Hudischewskyj, A.B., Seinfeld, J.H., Whitby, K.T., Whitby, E.R., Brock, J.R. and Barnes, H.M., Aerosol Sci. Technol. 5, 205(1986).

[43] Smolarkiewicz, P.K., Mon. Wea. Rev. 111, 479(1983).

[44] Smolarkiewicz, P.K., J. Comp. Phys. 54, 325(1984).

[45] Stroud, A.H., Approximate Calculation of Multiple Integrals, PrenticeHall, Englewood Cliffs(1971).

[46] Suck, S.H. and Brock, J.R., J. Aerosol Sci. 10, 581(1979).

[47] Tesche, T.W., Mathl Modelling 9, 507(1987).

[48] Tsang, T.H. and Brock, J.R., Atmos. Environ. 16, 2229(1982).

[49] Tsang, T.H. and Brock, J.R., Aerosol Sci. Technol. 2, 311(1983).

[50] Tsang, T.H. and Brock, J.R., Aerosol Sci. Technol. 3, 283(1984).

[51] Tsang T.H. and Hippe, J.N., Aerosol Sci. Technol. 8, 265(1988).

[52] Tsang T.H. and Korgaonkar, N., Aerosol Sci. Technol. 7, 317(1987).

[53] Varog̃lu, E. and Liam Finn, W.D., J. Comp. Phys. 34, 371(1980).

[54] Warren, D.R., Ph. D. Thesis, California Institute of Technology, Pasadena(1986).

[55] Warren, D.R. and Seinfeld, J. H., Aerosol Sci. Technol. 4, 31(1985).

[56] Wu, J.J. and Flagan, R.C., J. Colloid Interface Sci. 123, 339(1988).

[57] Yamartino, R.J., Scire, J.R., Hanna, S.R., Carmichael, G.R and Chang, Y.S., California Air Resources Board Report No. A049-1 (1989). 
[58] Yanenko, N.N., The Method of Fractional Steps, Springer-Verlag, New York(1971).

[59] Young., T.R., Navel Research Laboratory Memorandum Report 40912 (1980).

[60] Young, T.R. and Boris, J.P., J. Phys. Chem. 81, 2424(1977).

[61] Zalesak, S.T., J. Comp. Phys. 31, 335(1979). 
Table 1. Abbreviations of Numerical Methods

for the Advection Equation

Method

Abbreviation

Galerkin Finite Element Method

G-FEM

or Chapeau function method [29]

Finite Element Method with Forester's Method [12, 31] F-FEM

One Dimensional Flux-Corrected Transport

SHASTA

(FCT) Method [2, 3]

Generalized FCT Method [61]

G-FCT

Repeated Upwind Difference Method [43, 44]

SMO

Total Variation Diminishing Method [19, 20]

TVD 
Table 2. Recent Tests of Advection Schemes

\begin{tabular}{|c|c|c|c|}
\hline Authors & $\begin{array}{l}\text { Number of } \\
\text { methods } \\
\text { compared }\end{array}$ & $\begin{array}{l}\text { Model } \\
\text { problems }\end{array}$ & $\begin{array}{l}\text { Suggested } \\
\text { best } \\
\text { methods }\end{array}$ \\
\hline $\begin{array}{l}\text { Long and } \\
\text { Pepper[30] }\end{array}$ & 6 & $\begin{array}{l}2 \mathrm{D} \text { rotating } \\
\text { cosine hill }\end{array}$ & $\begin{array}{l}\text { Cubic spline method } \\
\text { Second moment method } \\
\text { G-FEM }\end{array}$ \\
\hline $\begin{array}{l}\text { McRae } \\
\text { et al. }[31]\end{array}$ & 6 & $\begin{array}{l}\text { 1D square } \\
\text { triangle } \\
\text { Gaussian }\end{array}$ & F-FEM \\
\hline $\begin{array}{l}\text { Chock and } \\
\text { Dunker }[8]\end{array}$ & 6 & $\begin{array}{l}2 \mathrm{D} \text { rotating } \\
\text { cosine hill }\end{array}$ & $\begin{array}{l}\text { G-FEM } \\
\text { G-FCT }\end{array}$ \\
\hline $\begin{array}{l}\text { Smolarkiewicz } \\
{[44]}\end{array}$ & 3 & $\begin{array}{l}2 \mathrm{D} \text { rotating } \\
\text { cosine hill } \\
3 \mathrm{D} \text { rotating } \\
\text { sphere }\end{array}$ & SMO \\
\hline Chock [7] & 5 & $\begin{array}{l}2 \mathrm{D} \text { rotating } \\
\text { cosine hill }\end{array}$ & F-FEM \\
\hline $\begin{array}{l}\text { Oran and } \\
\text { Boris [34] }\end{array}$ & 8 & $\begin{array}{l}\text { 1D square } \\
\text { Gaussian } \\
\text { Half dome }\end{array}$ & No conclusion \\
\hline Tesche [47] & 2 & $\begin{array}{l}\text { Urban air } \\
\text { data }\end{array}$ & G-FCT \\
\hline Hov et al. [26] & 4 & $\begin{array}{l}\text { Hypothetical } \\
\text { air data }\end{array}$ & $\begin{array}{l}\text { Pseudospectral method } \\
\text { G-FEM }\end{array}$ \\
\hline $\begin{array}{l}\text { Yamartino } \\
\text { et al. [57] }\end{array}$ & 4 & $\begin{array}{l}2 \mathrm{D} \text { rotational } \\
\text { cosine hill } \\
\text { A point source }\end{array}$ & Modified F-FEM \\
\hline
\end{tabular}


Table 3. Properties of Numerical Methods for Advection

\begin{tabular}{|c|c|c|}
\hline Method & Strengths & Limitations \\
\hline G-FEM [29] & Accuracy & $\begin{array}{l}\text { Dispersion errors } \\
\text { (negativity) }\end{array}$ \\
\hline F-FEM $[12,31]$ & $\begin{array}{l}\text { Positivity } \\
\text { Accuracy }\end{array}$ & $\begin{array}{l}\text { Ad hoc method } \\
\text { Diffusion errors }\end{array}$ \\
\hline Asymmetric G-FEM [29] & $\begin{array}{l}\text { Upwind property } \\
\text { (positivity) } \\
\text { Accuracy }\end{array}$ & Diffusion errors \\
\hline SHASTA $[2,3]$ & $\begin{array}{l}\text { Positivity } \\
\text { Easy to use }\end{array}$ & $\begin{array}{l}\text { Diffusion errors } \\
\text { One dimensional scheme }\end{array}$ \\
\hline $\mathrm{G}-\mathrm{FCT}[61]$ & $\begin{array}{l}\text { Positivity } \\
\text { Accuracy }\end{array}$ & $\begin{array}{l}\text { Diffusion errors } \\
\text { Slow speed }\end{array}$ \\
\hline $\mathrm{SMO}[43,44]$ & $\begin{array}{l}\text { Positivity } \\
\text { Accuracy } \\
\text { Easy to use }\end{array}$ & Diffusion errors \\
\hline $\operatorname{TVD}[19,20]$ & $\begin{array}{l}\text { Positivity } \\
\text { Accuracy }\end{array}$ & Diffusion errors \\
\hline
\end{tabular}


Table 4. Comparison of Numerical Methods on a Model Problem:

Two-Dimensional Rotating Cosine Hill $[7,8]$

\begin{tabular}{lllll} 
Items $^{1}$ & SHASTA & G-FCT $^{2}$ & G-FEM $^{2}$ & F-FEM $^{2}$ \\
\hline & & & & \\
Peak (100) & 27 & $50 / 58$ & $87 / 91$ & $76 / 71$ \\
Minimum(0) & 0 & $0 / 0$ & $-5 /-20$ & $-1 / 0$ \\
$\begin{array}{l}\text { Mass distribution } \\
\text { ratio (1) }\end{array}$ & 0.33 & $0.51 / 0.61$ & $0.8 / 1.0$ & $0.74 / 0.51$ \\
$\begin{array}{l}\text { Average absolute } \\
\text { error (0) }\end{array}$ & 1.3 & $0.8 / 0.6$ & $0.7 / 1.1$ & $0.65 / 0.8 .5$ \\
& & & & \\
$\begin{array}{l}\text { Computing } \\
\text { Time(s) }\end{array}$ & 44 & $140 / 135$ & $44 / 51$ & $65 / i 1$ \\
& & & & \\
Memory size (2D) & 4 & $8 / 12$ & $3 / 3$ & $3 / 3$ \\
\hline
\end{tabular}

${ }^{1}$ Numbers in parentheses are values of the exact solution.

${ }^{2}$ Different values mean different time integration schemes. 
Table 5. Comparison of Numerical Methods on a Model Problem:

Two-Dimensional Rotating Cosine Hill $[43,44]$

\begin{tabular}{lllll} 
Items $^{1}$ & Upwind & G-FCT & TVD $^{2}$ & SMO $^{3}$ \\
\hline & & & & \\
Peak(1) & 0.07 & 0.79 & $0.41 / 0.38$ & $0.54 / 0.81$ \\
Minimum(0) & 0 & $-1 \times 10^{-15}$ & $0 / 0$ & $0 / 0$ \\
Computing & 1 & 7.7 & $3 / 3$ & $2.9 / 7$ \\
Time(ratio) & & & & \\
& & & & \\
Conservation & 0.95 & 0.29 & $0.52 / 0.60$ & $0.52 / 0.14$ \\
Error $(0)^{4}$ & & & & \\
& & & & \\
\hline
\end{tabular}

1 Numbers in parentheses are values of the exact solution.

${ }^{2}$ First values: time splitting, second values: combined form.

${ }^{3}$ First values: two iterations, second values: four iterations.

${ }^{4}$ Conservation Error is defined as:

$$
1-\frac{\int\left(\text { sizedistribution }\left.\right|_{t=t}\right)^{2} d V+\int(\text { outflow }) d t}{\int\left(\text { sizedistribution }\left.\right|_{t=0}\right)^{2} d V} .
$$


Table 6. Numerical Methods for Advection Processes Used in Current Grid-Based Photochemical Air Quality Models

Air Quality Model Method

UAM (Systems Application, Inc.) SMO

CIT (California Institute of Technology F-FEM and Carnegie-Mellon University)

CALGRID (State of California Air Resources

Modified F-FF:H

Board and Sigma Corp.) 
Table 7. Numerical I'ethods for Stiff ODEs

\begin{tabular}{|c|c|c|}
\hline Method & Strengths & Limitations \\
\hline $\begin{array}{l}\text { Backward differentiation } \\
\text { formulae (BDF) }[18,34]\end{array}$ & $\begin{array}{l}\text { Widely tested } \\
\text { Rigorous } \\
\text { Accurate }\end{array}$ & $\begin{array}{l}\text { Multistep method } \\
\text { Matrix operations } \\
\text { Slow speed }\end{array}$ \\
\hline $\begin{array}{l}\text { Asymptotic integration } \\
\text { methods }[34,59,60]\end{array}$ & $\begin{array}{l}\text { No matrix operation } \\
\text { One step method } \\
\text { Fast }\end{array}$ & $\begin{array}{l}\text { Second order accurate } \\
\text { No conservation of mass }\end{array}$ \\
\hline $\begin{array}{l}\text { (Semi-)Implicit } \\
\text { Runge-Kutta } \\
\text { methods [18] }\end{array}$ & $\begin{array}{l}\text { Widely tested } \\
\text { Rigorous } \\
\text { Accurate }\end{array}$ & $\begin{array}{l}\text { Matrix operatiori- } \\
\text { Slow speed }\end{array}$ \\
\hline $\begin{array}{l}\text { Exponentially-fitted } \\
\text { methods }[34,37]\end{array}$ & $\begin{array}{l}\text { One step method } \\
\text { Comparable to BDF }\end{array}$ & $\begin{array}{l}\text { Usually needs } \\
\text { matrix operations } \\
\text { Slow }\end{array}$ \\
\hline $\begin{array}{l}\text { (Semi-)Implicit } \\
\text { extrapolation } \\
\text { methods }[11,18,34]\end{array}$ & Accurate & Matrix operations \\
\hline $\begin{array}{l}\text { Quasi-steady state } \\
\text { approximation } \\
\text { methods }[22,57]\end{array}$ & $\begin{array}{l}\text { One step method } \\
\text { Fast speed }\end{array}$ & $\begin{array}{l}\text { QSSA } \\
\text { Step size limitations } \\
\text { Low accuracy }\end{array}$ \\
\hline $\begin{array}{l}\text { (Semi-)Implicit } \\
\text { Euler methods [38] }\end{array}$ & $\begin{array}{l}\text { One step method } \\
\text { Fast speed } \\
\text { No matrix operations }\end{array}$ & Low accuracy \\
\hline
\end{tabular}


Table 8. Integration Packages for Stiff ODEs

Package Numerical methods Remark

EPISODE [25], Implicit Adams method (nonstiff) Rigorous

GEAR [23], BDF (stiff) Well tested

LSODE [24] Slow speed

CHEMEQ Hybrid predictor-corrector method Fast

$[34,59,60] \quad$ Euler method (nonstiff) Second-order accuracy

Asymptotic method (stiff)

LARKIN [11] Semi-implicit mid-point rule Comparable to GEAR

(Extrapolation method)

CREK1T [37] Exponentially fitted Especially for trapezoidal method combustion 
Table 9. Numerical Methods for Chemical Kinetics Used in Grid-Based Photochemical Air Quality Models

Air Quality Model

UAM (Systems Application, Inc.)

CIT (California Institute of Technology and Carnegie-Mellon University)

CALGRID (State of California Air Resources

Board and Sigma Corp.)
Method

Crank-Nicolson method

CHEMEQ

CHEMEQ, QSSA 
Table 10. Summary of Numerical Technique in Current Grid-Based Photochemical Air Quality Models

\begin{tabular}{llll} 
Component & UAM & CALGRID & CIT \\
\hline $\begin{array}{lll}\text { Operator } \\
\text { Splitting }\end{array}$ & Yes & Yes & yes \\
Advection & SMO & Modified F-FEM & F-FEM \\
$\begin{array}{l}\text { Vertical } \\
\text { Diffusion }\end{array}$ & Crank-Nicholson & $\begin{array}{l}\text { Crank-Nicholson } \\
\text { and Implicit }\end{array}$ & $\begin{array}{l}\text { G-FEM with } \\
\text { Crank-Nicholson } \\
\text { finite difference }\end{array}$ \\
time integration \\
Chemistry & Crank-Nicholson & $\begin{array}{l}\text { PSSA } \\
\text { Either QSSA } \\
\text { or CHEMEQ }\end{array}$ & $\begin{array}{l}\text { PSSA } \\
\text { QSSA }\end{array}$ \\
& & &
\end{tabular}


Table 11. Existing Aerosol Model Based on the General Dynawic Equation

\begin{tabular}{|c|c|c|c|c|c|}
\hline Author & Process ${ }^{1}$ & $\begin{array}{l}\text { No. of } \\
\text { comp. }^{2}\end{array}$ & Application ${ }^{3}$ & $\begin{array}{l}\text { Numerical } \\
\text { method }\end{array}$ & $\begin{array}{l}\text { ity for } \\
\text { deling }\end{array}$ \\
\hline $\begin{array}{l}\text { Middleton and } \\
\text { Brock[32] }\end{array}$ & $\begin{array}{l}\mathrm{CD} \\
\mathrm{CG} \\
\mathrm{N}\end{array}$ & S & $\mathrm{H}$ & $\begin{array}{l}\text { J-space transform, } \\
\text { Cubic spline, } \\
\text { Runge-Kutta }\end{array}$ & $\mathrm{N} / \mathrm{A}$ \\
\hline $\begin{array}{l}\text { Gelbard and } \\
\text { Seinfeld[13] }\end{array}$ & $\begin{array}{l}\mathrm{CD} \\
\mathrm{CG} \\
\mathrm{S}, \mathrm{R}\end{array}$ & $S$ & $\mathrm{H}$ & $\begin{array}{l}\text { Finite element } \\
\text { with orthogonal } \\
\text { collocation and } \\
\text { cubic spline }\end{array}$ & $\because / A$ \\
\hline $\begin{array}{l}\text { Suck and } \\
\text { Brock[46] }\end{array}$ & CG & S & $\mathrm{H}$ & $\begin{array}{l}\text { J-space transiorm. } \\
\text { Cubic spline, } \\
\text { Adams-Moulton }\end{array}$ & $\because / \mathrm{A}$ \\
\hline $\begin{array}{l}\text { Gelbard } \\
\text { et al.[15] }\end{array}$ & $\mathrm{CG}$ & $S$ & Plumes & Sectional method & $\therefore / \mathrm{A}$ \\
\hline $\begin{array}{l}\text { Gelbard and } \\
\text { Seinfeld[14] }\end{array}$ & $\begin{array}{l}\text { CG, CD, } \\
\mathrm{S}, \mathrm{R}\end{array}$ & $\mathrm{M}$ & $\begin{array}{l}\text { Urban } \\
\text { plume }\end{array}$ & Sectional method & AIP \\
\hline $\begin{array}{l}\text { Bassett } \\
\text { et al.[1] }\end{array}$ & $\begin{array}{l}\text { CD, CG, } \\
\text { CR, S }\end{array}$ & $\mathrm{M}$ & Plumes & Sectional method & AIP \\
\hline $\begin{array}{l}\text { Tsang and } \\
\text { Brock[48] }\end{array}$ & CG & $S$ & Plumes & $\begin{array}{l}\text { Methods of Suck } \\
\text { and Brock[46] }\end{array}$ & $\mathrm{N} / \mathrm{A}$ \\
\hline $\begin{array}{l}\text { Tsang and } \\
\text { Brock }[49,50]\end{array}$ & $\mathrm{CD} / \mathrm{EV}$ & $\mathrm{S}$ & $\mathrm{H}$ & $\begin{array}{l}\text { Finitc element with } \\
\text { characteristics, } \\
\text { Cubic spline }\end{array}$ & $\mathrm{N} / \mathrm{A}$ \\
\hline $\begin{array}{l}\text { Warren and } \\
\text { Seinfeld[55] }\end{array}$ & $\begin{array}{l}\mathrm{CD} / \mathrm{EV}, \\
\mathrm{CG}, \\
\mathrm{N}, \mathrm{D}\end{array}$ & S & $\mathrm{H}$ & Sectional method & $\mathrm{N} / \mathrm{A}$ \\
\hline
\end{tabular}


Table 11. (continued)

\begin{tabular}{|c|c|c|c|c|c|}
\hline Author & Process $^{1}$ & $\begin{array}{l}\text { No. of } \\
\text { comp. }^{2}\end{array}$ & Application $^{3}$ & $\begin{array}{l}\text { Numerical } \\
\text { method }\end{array}$ & $\begin{array}{l}\text { ility for } \\
\text { odeling }\end{array}$ \\
\hline $\begin{array}{l}\text { Pilinis } \\
\text { et al. }[35,36]\end{array}$ & $\begin{array}{l}\mathrm{CD} / \mathrm{EV}, \\
\mathrm{CG}, \mathrm{R}, \\
\mathrm{S}(\mathrm{N})\end{array}$ & M & $\begin{array}{l}\text { Air } \\
\text { trajectory } \\
\text { model }\end{array}$ & Sectional method & DA \\
\hline Gelbard[16] & $\begin{array}{l}\mathrm{CD} / \mathrm{EV}, \\
\mathrm{CG}, \mathrm{S}\end{array}$ & M & $\begin{array}{l}\text { Nuclear } \\
\text { reactor }\end{array}$ & $\begin{array}{l}\text { Moving and } \\
\text { fixed } \\
\text { sectional method }\end{array}$ & AIP \\
\hline $\begin{array}{l}\text { Brock and } \\
\text { Oates[4] }\end{array}$ & $\mathrm{EV}$ & S & $\mathrm{H}$ & Moment method & $\therefore A$ \\
\hline $\begin{array}{l}\text { Tsang and } \\
\text { Korgaonkar[52] }\end{array}$ & EV & $\mathrm{S}$ & $\mathrm{H}$ & $\begin{array}{l}\text { Galerkin finite } \\
\text { element and } \\
\text { Smolarkiewicz } \\
\text { method }\end{array}$ & $\therefore / A$ \\
\hline $\begin{array}{l}\text { Wu and } \\
\text { Flagan[56] }\end{array}$ & $\begin{array}{l}\mathrm{CR}, \\
\mathrm{CG}, \\
\mathrm{CD} / \mathrm{EV}\end{array}$ & $\mathrm{S}$ & $\begin{array}{l}\text { Aerosol } \\
\text { generating } \\
\text { experiment }\end{array}$ & $\begin{array}{l}\text { Discrete } \\
\text { sectional } \\
\text { method }\end{array}$ & $\mathrm{N} / \mathrm{A}$ \\
\hline $\begin{array}{l}\text { Tsang and } \\
\text { Hippe[51] }\end{array}$ & $\begin{array}{l}\mathrm{CD} / \mathrm{EV} \\
\mathrm{CG}\end{array}$ & $\mathrm{S}$ & $\mathrm{H}$ & $\begin{array}{l}\text { Methods of } \\
{[46] \text { and [49] }}\end{array}$ & $\mathrm{N} / \mathrm{A}$ \\
\hline Gelbard[17] & $\mathrm{CD} / \mathrm{EV}$ & $\mathrm{M}$ & $\mathrm{H}$ & $\begin{array}{l}\text { Moving sectional } \\
\text { method }\end{array}$ & AIP \\
\hline $\begin{array}{l}\text { Kim and } \\
\text { Seinfeld[28] }\end{array}$ & $\mathrm{CD} / \mathrm{EV}$ & M & $\mathrm{H}$ & $\begin{array}{l}\text { Moving sectional } \\
\text { method }\end{array}$ & AIP \\
\hline
\end{tabular}

${ }^{1} \mathrm{CD}$ : condensation, EV: evaporation, CG: coagulation, S: source, $\mathrm{R}$ : removal, $\mathrm{N}$ : nucleation, $\mathrm{D}$ : deposition, $\mathrm{CR}$ : chemical reaction.

${ }^{2} \mathrm{~S}$ : single component, $\mathrm{M}$ : multicomponent.

${ }^{3} \mathrm{H}$ : hypothetical case.

${ }^{4} \mathrm{~N} / \mathrm{A}$ : not applicable, API: applicable in principle, DA: directly applicable. 


\section{FIGURE CAPTION}

Figure 1. Schematic showings of the numerical diffusion and dispersion errors. Solid lines: exact solutions, broken lines: numerical solutions. 
NUMERICAL DIFFUSION

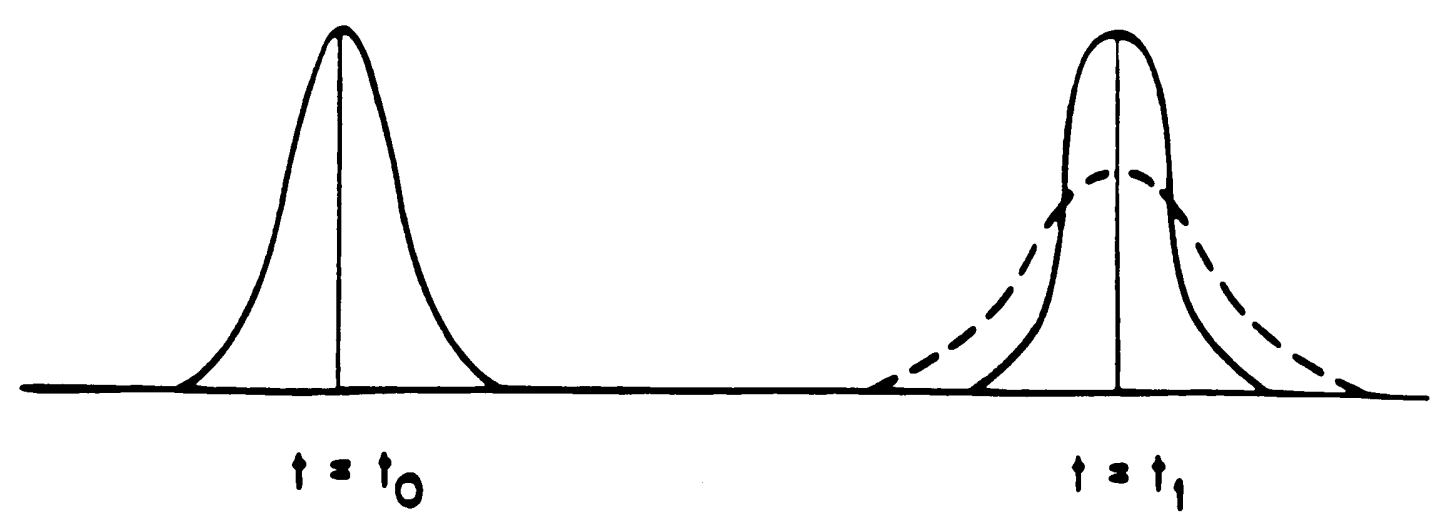

NUMERICAL DISPERSION

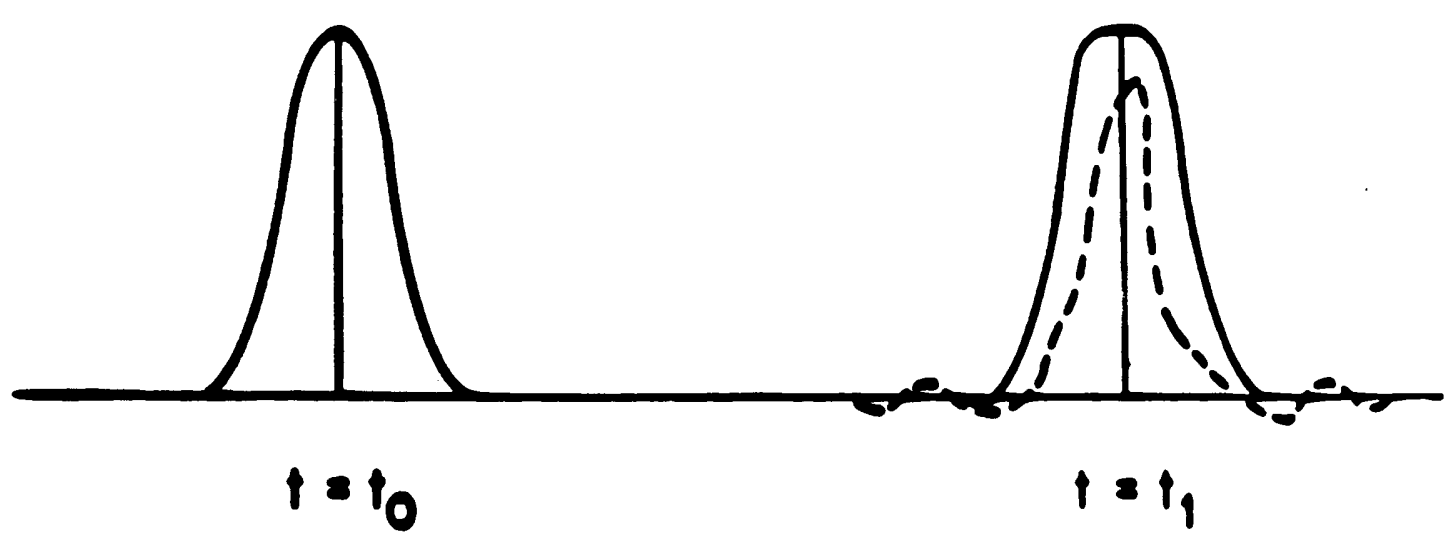

Figure 1 


\title{
APPENDIX II
}

\section{AMPS VERSION 1.0 USER'S MANUAL}

\author{
Yong Pyo Kim
}

Department of Chemical Engineering

California Institute of Technology

Pasadena, CA 91125

July, 1991 


\section{INTRODUCTION}

The main objective of AMPS (arbitrarily mixed particles simulator) is to simulate multicomponent aerosol dynamics with simultaneous processes without any assumpton on tile nature of the distribution. Therefore, it can simulate the number-chemical composition distribution. For a detailed description of the routine, see Kim and Seinfeld (1).

This program is written by Yong Pyo Kim in CALTECH during 1989-1990. The language used is FORTRAN used at SUN SPARC station but this routine can be used in other computers with slight modifications. Since the program is written wi! a modular approach, a use can modify the source program easily.

It can simulate following processes: (1) coagulation, (2) condensation/evaporat .... (3) depositional loss, and (4) time varying source and nucleation.

In Section 2, processes, mechanisms, and numerical schemes are describer $1: 1$ Secion 3, input data file is explained and an example is given.

Main variables are explained in main program, main.f and brief descriptions fi, individual programs are also given in each program.

\section{PROCESSES AND MECHANISMS}

At present, following options are available for processes and formulae for some mechanisms are shown (options in parenthesis will be added later). Also, numerical schemes used are summarized in Table I.

- Coagulation

1. Constant coefficeint

2. Brownian diffusion coefficient

3. (Turbulence coagulation coefficient)

4. (Gravity coagulation coefficient) 
- Condensation/evaporation

1. Diffusion-controlled or Maxwell relation

2. (Surface reaction-controlled)

3. (Volume reaction-controlled)

4. Volume reaction-controlled with a constant

- Source

1. User given data file

2. Exponential distribution

3. Log-normal distribution

4. User given subroutine

- Deposition

1. Gravity settling deposition

2. (Phoretic effects)

- Nucleation: User given time dependent nucleation rates and location

Also, the inital distribution has following options:

1. User given data file

2. Exponential distribution

3. Log-normal distribution

4. User given subroutine

2.1 Condensation/Evaporation 
Two mechanisms are considered in the routine, one is the diffusion-controlled gas-to-particles conversion, also known as the Maxwell relation and the other is the volume reaction-controlled gas-to-particles conversion (2). The Maxwell relation is the most widely used relation and is in the form;

$$
I_{i}=\frac{d m_{i}}{d t}=2 \pi D_{p} D_{i}\left(c_{i}-c_{i e}\right) \beta_{F}
$$

where $I_{i}$ is the condensation/evaporation rate of the $i$-th component, $m_{i}$ and $D_{i}$ the mass and the diffusivity of $i$-th component, respectively. $D_{p}$ is the diameter of a particle, $c_{i}$ and $c_{i e}$ the mass concentration of $i$-th component in the gas phase and in equilibrium state, respectively, and $\beta_{F}$ is the correction factor by Fuchs and Stugin (2) and given by

$$
\begin{gathered}
\beta_{F}=\frac{1+\mathrm{Kn}}{1+1.71 \mathrm{Kn}+1.33 \mathrm{Kn}^{2}} \\
\mathrm{Kn}=2 \lambda / \mathrm{D}_{\mathrm{p}}
\end{gathered}
$$

where $\mathrm{Kn}$ is the Knudsen number and $\lambda$ is the mean free path of the air.

For aerosol particles of submicron size, the Kelvin effect ( 2 may be significant. Due to the curvature of a particle, the partial pressure on the aerosol surface is higher than that on the flat bulk surface of the same composition. The relation in concentration form is

$$
\frac{c_{i e}}{c_{i e, b}}=\ln \left(\frac{4 \pi \sigma \overline{v_{i}}}{R T D_{p}}\right)
$$

where $c_{i e}$ and $c_{i e, b}$ are the equilibrium concentration on the aerosol surface and the flat bulk surface, respectively. $\sigma$ is the surface tension of an aerosol, $\overline{v_{i}}$ is the molar liquid volume of the species $i, R$ is the gas constant $(8.314 \mathrm{~J} /$ mole $\mathrm{K})$, and $T$ is the absolute temperature.

The volume reaction-controlled gas-to-particles conversion is expressed as,

$$
I_{i}=\frac{d m_{i}}{d t}=\sigma_{i} m_{i}
$$

where $\sigma_{i}$ is the rate constant of $i$-th component. In this case, no detailed calculation of $\sigma_{i}$ is done. The purposes of this option are twofold, firsty to simulate the 
volume reaction-controlled gas-to-particles conversion mechanism in a simplified way, secondly to check the performance of the routine since analytical solutions for this rate form can be obtained for many cases including for simultaneous processes.

\subsection{Coagulation}

Coagulation due to the Brownian diffusion (the Fuchs form) is used (2),

$$
\begin{gathered}
\beta_{12}=2 \pi\left(D_{1}+D_{2}\right)\left(\gamma_{1} D_{p 1}+\gamma_{2} D_{p 2}\right)\left[\frac{D_{p 1}+D_{p 2}}{D_{p 1}+D_{p 2}+2 g_{12}}+\frac{8\left(D_{1}+D_{2}\right)}{\bar{c}_{12}\left(D_{p 1}+D_{p 2}\right)}\right]^{-1} \\
D_{i}=\frac{k T}{3 \pi \mu D_{p i}}\left[\frac{5+4 \mathrm{Kn}_{\mathrm{i}}+6 \mathrm{Kn}_{\mathrm{i}}{ }^{2}+18 \mathrm{Kn}_{\mathrm{i}}{ }^{3}}{5-\mathrm{Kn}_{\mathrm{i}}+(8+\pi) \mathrm{Kn}_{\mathrm{i}}{ }^{2}}\right] \\
g_{12}=\left(g_{1}^{2}+g_{2}^{2}\right)^{1 / 2} \\
g_{i}=\left(1 /\left(3 D_{p i} l_{i}\right)\right)\left[\left(D_{p i}+l_{i}\right)^{3}-\left(D_{p i}^{2}+l_{i}^{2}\right)^{3 / 2}\right]-D_{p i} \\
l_{i}=8 D_{i} / \pi \bar{c}_{i} \\
\bar{c}_{12}=\left(\bar{c}_{1}^{2}+\bar{c}_{2}^{2}\right)^{1 / 2} \\
\bar{c}_{i}=\left(8 k T / \pi m_{i}\right)^{1 / 2}
\end{gathered}
$$

where $\beta_{i j}$ is the coagulation coefficient between particles $i$ and $j, \gamma_{i}$ is the agglomeration shape factor, $\mu$ is the gas viscosity, and $k$ is the Boltzmann's constant.

Constant coagulation coefficient is useful for model validation and a quick process assessment.

\subsection{Deposition}

The linear deposition model due to gravity which is similar to that used in MAEROS (3) but the density difference correction is added, is used here.

$$
\begin{gathered}
R[\mathbf{m}, t, n(\mathbf{m}, t)]=\frac{\text { floor area }}{\text { volume }} v_{d} n(\mathbf{m}, t) \\
v_{d}=\frac{\left(\rho_{p}-\rho_{g}\right) g D_{p}^{2} C_{d}}{18 \chi \mu}
\end{gathered}
$$


where $v_{\boldsymbol{d}}$ is the deposition velocity, $\rho_{p}$ and $\rho_{g}$ the density of a particle and gas, respectively, $\mathrm{g}$ is the gravity constant, $C_{d}$ is the Cunningham correction factor,

$$
C_{d}=1+\mathrm{Kn}[1.37+0.4 \exp (-1.1 / \mathrm{Kn})]
$$

and $\chi$ is a shape correction factor to account for the non-spherical effects.

\subsection{Source and Nucleation}

The source rates and distributions should be given by a user. Both exponential and log-normal distribution are installed in the routine as a choice of the source distribution or a user can input a distribution. Since the existing multicomponent nucleation theory is not well established to predict accurate nucleation rates and/or size, and composition, these should be also given by a user.

\section{INPUT DATA FILE}

Input data file description is given and some explanation is needed; $i$ and $e$ in bracket stand for an integer and a real values, respectively; and the expression $i=1, n x x x x$ stands for the repeated reading of (nxxxx) times. Also, an example of the input data file is given in Table II.

- line 1 : title, up to 79 characters

- line 2 : blank ncomp[i]: number of component

- line 3 : blank $\mathrm{i}=1$, ncomp

1. ngrid[i] : number of grid

2. ngaus[i] : number of Gaussian quadrature point

3. da $[\mathrm{e}]$ : lower calculational domain $(\mathrm{kg})$ 
4. $\mathrm{db}[\mathrm{e}]$ : upper calculational domain $(\mathrm{kg})$

5. dens[e] : density of $\mathrm{i}$-th component $(\mathrm{kg} / \mathrm{m} 3)$

6. wtmol[e] : molecular weight $(\mathrm{kg} /$ mole $)$

7. congas[e]: initial gas concentration $(\mathrm{kg} / \mathrm{m} 3)$

- line 4 : blank

1. $\operatorname{totm}[\mathrm{e}]$ : initial total mass concentration $(\mathrm{kg} / \mathrm{m} 3)$

2. $\operatorname{totn}[\mathrm{e}]:$ initial total number concentration $(1 / \mathrm{m} 3)$

- line 5 : blank

1. $\operatorname{dt}[\mathrm{e}]$ : suggested temporal $\operatorname{step}(\mathrm{s})$, in nuint.f, this value is compared to the characteristic times of processes and the minimum value is used as the final temporal step

2. $\operatorname{tmax}[\mathrm{e}]:$ maximum time(s) for simulation

3. eps[e] : relative error bound

- line 6 : blank

1. iop[i] : option flag for condensation/evaportion and coagulation, if set zero, default value of 31 is used

- 11: pure cond/evap. with limited amount of condensable species

- 12: pure cond/evap. with unlimited amount of condensable species

- 20: pure coagulation

- 31: case of 11 and coagulation

- 32: case of 12 and coagulation. 
2. iini[i] : initial size distribution data,if set zero, default value of 3 is used

- 1: user given data

- 2: exponential initial distribution

- 3: log-normal initial distribution

- 4: user given subroutine.

3. ibound[i] : boundary condition, if set zero, default value of 2 is used

- 1: log-linear/symmetry

- 2: all set zero

- 3: user given subroutine.

4. icond[i] : condensation/evaporation rate, if set zero, default value of 1 is used

- 1: the repeated upwind difference method

- 2: the Taylor-Galerkin method

- 31: same as 1 but constant rates

- 41: same as 2 but constant rates

5. icoag[i] : coagulation rate, if set zero, default value of 2 is used

- 1: constant

- 2: Fucks relation.

6. isource[i]: source rate data, if set zero, default value of 3 is used

- 1: user given rate data

- 2: exponential source rate

- 3: log-normal source rate

- 4: user supplied soubourine.

7. inucl[i] : nucleation option flag 
- 0: no nucleation

- 1: nucleation

8. idepo[i] : deposition process

- 0: no deposition

- 1: deposition.

9. ikel[i] : Kelvin effect, if set zero, default value of 1 is used

- 1: no effect calculation

- 2: effect calculation.

- line 7 : blank

1. npt[i] : number of times results are printed.

2. npx[i] : number of grids results are printed.

3. nseg[i] : number of sections in sectional representation

4. iprint[i]: print option

- 1: print initial data, but mo number and mass moment calculation

- 2: print inital data and calculate number and mass moment by grid calculation, it takes lagre computing time

- 3: no initial data and no calculation

- 4: no initial data but calculation.

5. idebug[i]: report the status of the program

- 1: no report

- 2: basic report, mainly calling sequences

- 3: detailed report

- 4: in addition to 3 , report contributions of each process.

6. ntp[i] : number of temp. and pres. input, $0 ; i 21$ 
7. nsource[i]: number of source term input, $0=i 21$.

- line 8 : balnk

1. $s c[e]$ : scaling factor for the repeated upwind difference method.

2. iord[i] : number of iteration for the repeated upwind difference method.

- line 9 : blank

1. volume[e]: reactor volume $(\mathrm{m} 3)$

2. arce[e]g : ratio of ceiling area to volume $(1 / \mathrm{m})$

3. $\operatorname{arf}[\mathrm{e}] \mathrm{g}$ : ratio of floor area to volume $(1 / \mathrm{m})$

4. arwa[e]g : ratio of wall area to vlume $(1 / \mathrm{m})$

$* * * * * * * * * * * * * * * *$ arce and arwa are not used in this version

- line 10: blank

1. iplot[i] : plot sectional concentration

- -2: log-scale, user given boundaries

-1 : log-scale, automatic boundaries

- 10: no plot

- +1: linear scale, user given boundaries

- +2: linear scale, automatic boundaries.

2. cplot[i] : plot each component

-1 : no

-2 : yes.

3. nrow[i] : number of rows

4. ncol[i] : number of columns 
5. $x p l m i n[e]:$ minimum concentration $(\mathrm{kg} / \mathrm{m} 3)$

6. xplmax [e]: maximum concentration $(\mathrm{kg} / \mathrm{m} 3)$

$* * * * * * * * * * * * * * * * * *$ this line is not used in this version

- line 11: blank

$\mathrm{i}=1, \mathrm{ncomp}$

1. $x \operatorname{dm}[\mathrm{e}]: \mathrm{i}$-th component mass of mean diameter $(\mathrm{kg})$

2. $\operatorname{stdev}[\mathrm{e}]$ : standard deviation(meaningful only if iini $=3$ )

- line 12: blank

$\mathrm{i}=1, \mathrm{ntp}$

1. timetp[e]: time sequence(s)

2. temp[e]: temperature $(\mathrm{K})$

3. $\operatorname{pres}[\mathrm{e}]:$ pressure $(\mathrm{Pa}, 1 \mathrm{~Pa}=1 \mathrm{~N} / \mathrm{m} 2)$

4. $x$ nucl $[\mathrm{e}]$ : nucleation $\mathrm{rate}(1 / \mathrm{m} 3 / \mathrm{s})$, meaningful only if inucl $=1$

- line 13: this line is a part of line 12 , thus, (ncomp) lines of line 13 entry are needed for each entry of line 12 , and theie is no blank line $\mathrm{j}=1, \mathrm{ncomp}$

1. eqgas[e] : equilibrium gas concentration $(\mathrm{kg} / \mathrm{m} 3)$

2. $\operatorname{diff}[\mathrm{e}]:$ gas diffusivity $(\mathrm{m} 2 / \mathrm{s})$

3. $x n s[\mathbf{e}]$ : nucleation location in grid point $(\mathrm{kg}, \mathrm{kg}, \mathrm{kg}, \mathrm{kg}$ got 4 - component case)

- line 14:blank $\mathrm{i}=1$, nsource

1. timeso[e]: time of source data are given(s) 
2. sonum[e] : total number concentration of source $(1 / \mathrm{m} 3)$

- line 15: this line is a part of line 14, thus, (ncomp) lines of line 15 entry are needed for each entry of line 14 and there is no blank line $\mathrm{j}=1$, ncomp

1. $\operatorname{somam}[\mathrm{e}]: \mathrm{j}$-th component mass of mean diameter particle $(\mathrm{kg})$

2. $\operatorname{sodev}[\mathrm{e}]$ : standard deviation(meaningful only if isource $=3$ )

- line 16: blank this line is meaningful only if ikel $=1$ $\mathrm{i}=1$, ncomp

1. $\operatorname{sigma}[e]:$ sueface tension $(j / m)$

2. volliq[e]: liquid molar volume(m3/mole)

- line 17 :blank

this line is meaningful only if icoag $=1$ beta[e] : constant coagulation coefficeint $(\mathrm{m} 3 / \mathrm{s})$

- line 18:******* this is temporary input for constants of volume reactioncontrolled condensation/evaporation and has no blank line $\mathrm{i}=1$, ncomp $\operatorname{am}[e]: \operatorname{constant}(1 / \mathrm{s})$ 


\section{REFERENCES}

(1) Kim, Y.P.; Seinfeld, J.H. Aerosols: Science, Industry, Health and Environment; pp 138-141, S. Masuda; K. Takahashi Ed.; Pergamon Press: Oxford, 1990.

(2) Seinfeld, J.H. Atmospheric Chemistry and Physics of Air Pollution; John Wiley \& Sons: New York, 1986.

(3) Gelbard, F. MAEROS User Manual; NUREG/CR-1391, SAND80-0822, Sandia National Laboratories, 1982.

(3) Smolarkiewicz, P.K. Mon. Wea. Rev. 1983, 111, 479-486.

(4) Smolarkiewicz, P.K. J. Comp. Phys. 1984, 54, 325-362.

(5) Smolarkiewicz, P.K.; Clark, T.L. J. Comp. Phys. 1986, 67, 396-438.

(6) Donea, J. Int'l J. Numer. Meth. in Eng. 1984, 20, 101-119.

(7) Selmin, V.; Donea, J.; Quartrapelle, L. Compt. Meth. Appl. Mech. Eng. $1985,52,817-845$.

(8) Donea, J.; Quartapelle, L.; Selmin, V. J. Comp. Phys. 1987, 70, 463-499. 
Table I. Numerical Schemes Used in AMPS

\begin{tabular}{ll} 
Process & $\begin{array}{l}\text { Numerical } \\
\text { Scheme }\end{array}$ \\
\hline $\begin{array}{l}\text { Condensation/ } \\
\text { Evaporation }\end{array}$ & $\begin{array}{l}\text { Repeated upwind } \\
\text { difference (4-6), } \\
\text { Taylor-Galerkin (7-9) }\end{array}$ \\
Coagulation & $\begin{array}{l}\text { Product type } \\
\text { Gaussian quadrature }\end{array}$ \\
Deposition & With Coagulation \\
Source & With coagulation \\
Nucleation & With coagulation \\
Simultaneous & Operator splitting \\
Processes & \\
\hline
\end{tabular}


Table II. An Input Data File for AMPS

this is a test case for a two-comp. system

number of component

2 No. grid, Gaussian pt.,da,db, density, M.W.,gas conc.

$21,5,2.6 \mathrm{e}-19,2.6 \mathrm{e}-15,1 . \mathrm{e} 3,18 . \mathrm{e}-3,1 . e-5$

$21,5,2.6 \mathrm{e}-19,2.6 \mathrm{e}-15,1 . \mathrm{e} 3,28 . e-3,2 . e-5$

total mass and number

$5.2 \mathrm{e}-11,1 . \mathrm{e} 5$

delta (time step), maximum time, tolerance

$0.01,0.999,1.0 \mathrm{e}-4$

options

$20,2,3,2,1,2,0,0,0$

informations

$100,5,21,2,1,2,0$

cond/evap. parameters

$1.0,1$

chamber and physical parameters

$100.0, .1,0.2,0.3,1.0,1.0$

plot information

$-1,1,30,30, .0001,1.0$

initial distribution data

$2.60 \mathrm{e}-16,0.0$

$2.60 \mathrm{e}-16,0.0$

temperature, pressure table

$0.0,273,1 . e 5,0.0$

$.8 \mathrm{e}-5,1 . \mathrm{e}-6,0.0$

$.9 \mathrm{e}-5,1.2 \mathrm{e}-5,0.0$

$0.6,300 ., 1.2 \mathrm{e} 5,0.0$

$.82 \mathrm{e}-5,1.5 \mathrm{e}-6,0.0$

$.92 \mathrm{e}-5,1.7 \mathrm{e}-6,0.0$

source table

surface tension liquid molar volume

1.e3,2.e-2

$1.2 \mathrm{e} 3,1.2 \mathrm{e}-3$

constant coagulation coefficient

1.0

$.9,1.1$ 